\title{
MODELING OF A GYRO-STABILIZED HELICOPTER CAMERA SYSTEM USING NEURAL NETWORKS
}

\author{
A Thesis \\ presented to the faculty of the \\ California Polytechnic State University, San Luis Obispo \\ In Partial Fulfillment \\ of the Requirements for the Degree of \\ Master of Science in Electrical Engineering
}

by

Nicholas Layshot

December, 2010 
(C) 2010

Nicholas Layshot

All Rights Reserved 
TITLE:

AUTHOR:

DATE SUBMITTED:

COMMITTEE CHAIR:

COMMITTEE MEMBER:

COMMITTEE MEMBER:
Modeling of a Gyro-stabilized Helicopter Camera System using Neural Networks

Nicholas Layshot

December, 2010

Xiao-Hua Yu, Associate Professor

John A. Saghri, Associate Professor

Tina Smilkstein, Assistant Professor 


\begin{abstract}
Modeling of a Gyro-stabilized Helicopter Camera System using Neural Networks Nicholas Layshot

On-board gimbal systems for camera stabilization in helicopters are typically based on linear models. Such models, however, are inaccurate due to system nonlinearities and complexities. As an alternative approach, artificial neural networks can provide a more accurate model of the gimbal system based on their non-linear mapping and generalization capabilities.

This thesis investigates the applications of artificial neural networks to model the inertial characteristics (on the azimuth axis) of the inner gimbal in a gyro-stabilized multi-gimbal system. The neural network is trained with time-domain data obtained from gyro rate sensors of an actual camera system. The network performance is evaluated and compared with measured data and a traditional linear model. Computer simulation results show the neural network model fits well with the measured data and significantly outperforms a traditional model.
\end{abstract}




\section{ACKNOWLEDGEMENTS}

I would like to thank my family and friends for supporting me, and for encouraging me to strive to always better myself.

Dr. Helen Yu first introduced me to neural networks and has made this thesis possible. She has provided constant guidance throughout the thesis process, and I would like to thank her for the encouragement she has given me, and the productive discussions we have had.

From Axsys Technologies, I would like to thank everyone for their encouragement, especially John Coyle and Gene Katz for giving me the opportunity to work on this project. I would like to pay special attribute to Lafe Redd from Axsys Technologies for his constant support, encouragement and for providing the resources needed to complete this study. I would also like to thank Dr. Jim Hilkert of Alpha-Theta Technologies for his guidance and expertise on stabilized gimbal systems. 


\section{TABLE OF CONTENTS}

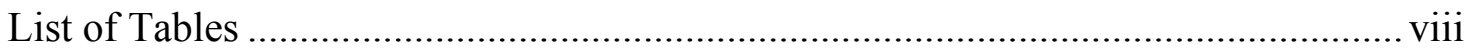

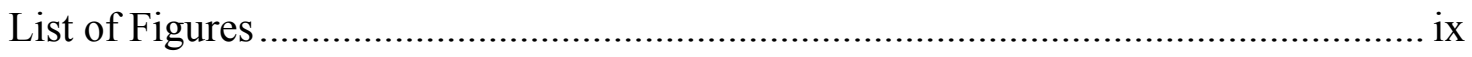

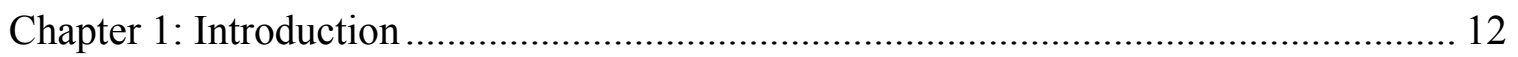

Chapter 2: Overview of Artificial Neural Networks...................................................... 28

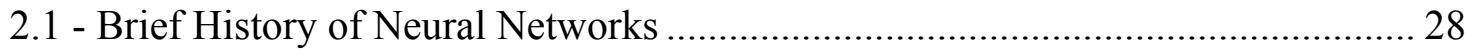

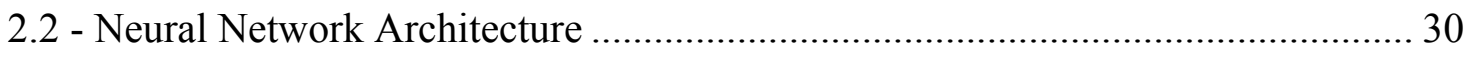

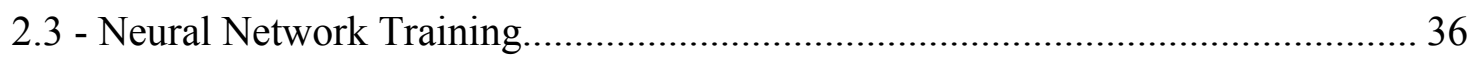

2.3.1 - Backpropagation Algorithm (BP) …………………………………..... 38

2.3.2 - Sequential vs. Batch Training .................................................................... 49

2.3.3 - Online vs. Offline Training …………....................................................... 49

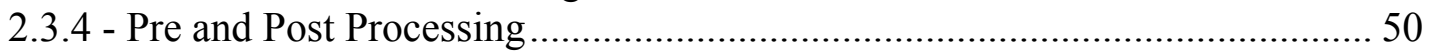

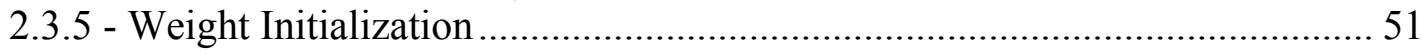

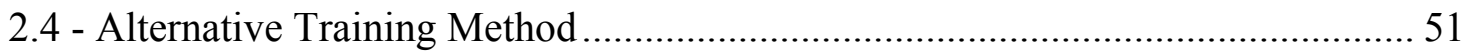

2.4.1 - Levenberg-Marquardt (LM) …………………..................................... 52

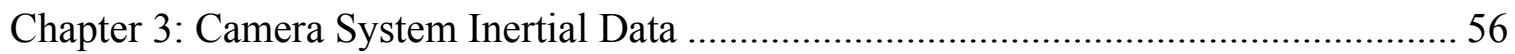

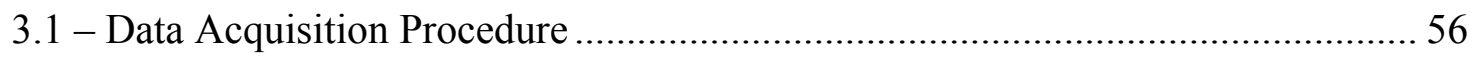

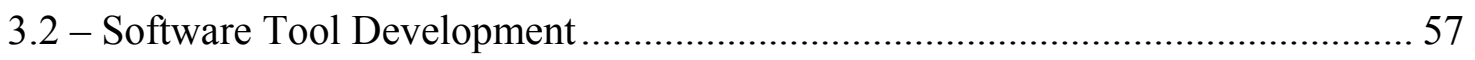

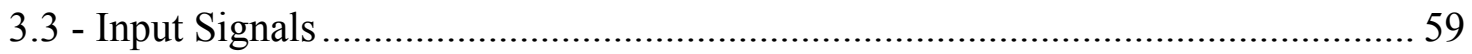

3.3.1 - Multiple Sine Waves Appended .................................................................... 60

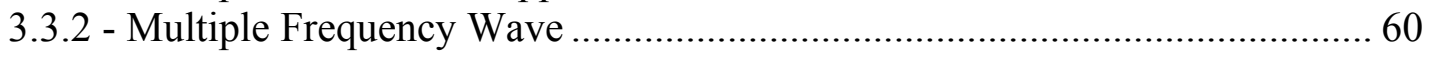

3.3.3 - Multiple Sine Waves Appended and Multiple Frequency Wave Appended. 61

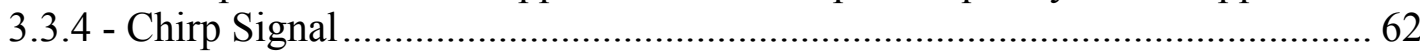

3.3.5 - Appended Multiple Random Amplitude \& Random Interval (ARARI) ....... 62

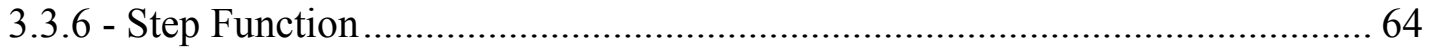

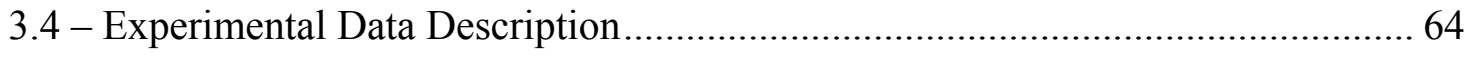

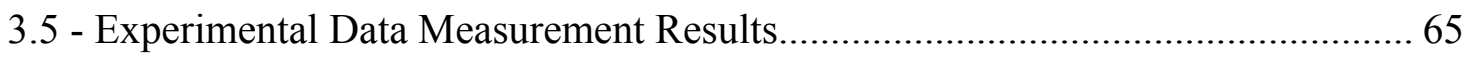

Chapter 4: Simulation Results .................................................................................. 78

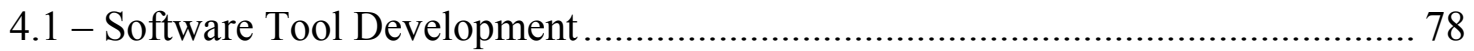

4.2 - Network Structure Comparison, Training and Simulation Results ..................... 82

4.2.1 Network Structure Description and Weight Initialization ................................ 83

4.2.2 Training Method ....................................................................................... 86 


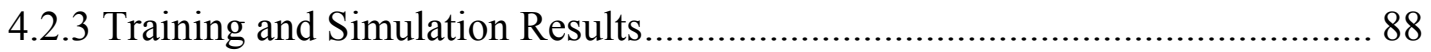

4.3 - Training and Simulation Results for Each Data Set ...................................... 101

4.4 - Network Generalization Study...................................................................... 113

Chapter 5: Traditional vs. Neural Network Model Comparison.................................... 128

5.1 - Traditional Model .................................................................................. 128

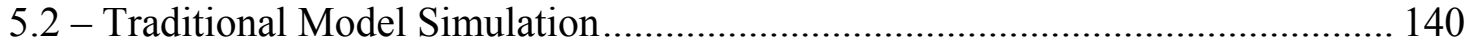

5.4 - Traditional vs. Neural Network Model Results ............................................ 148

Chapter 6: Conclusion and Future Works................................................................ 162

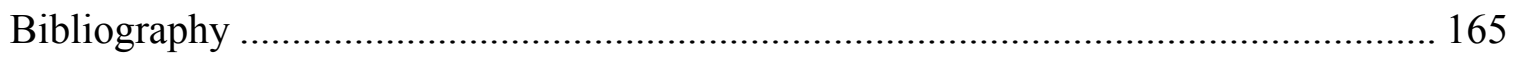

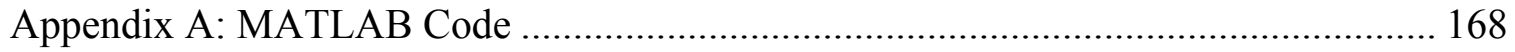




\section{List of Tables}

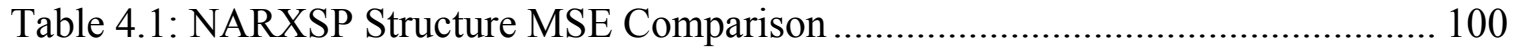

Table 4.2: NARXSP Structure R^2 Comparison............................................................ 100

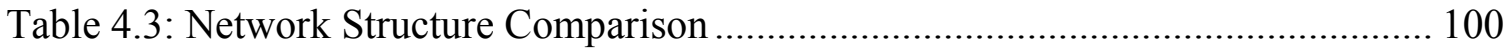

Table 4.4: Plant Modeling for Several Data Sets..................................................... 113

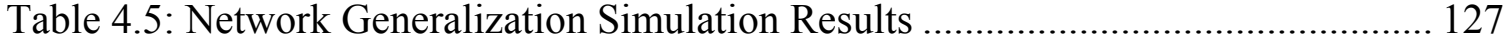

Table 5.1: Traditional vs. Neural Network Model Results ........................................ 161 


\section{List of Figures}

Figure 1.1: Gyro-stabilized Camera System ………………………………………... 12

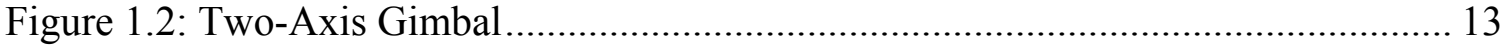

Figure 1.3: Model: Inner Gimbal Azimuth Axis .......................................................... 14

Figure 1.4: Motor and Amplifier Block Diagram....................................................... 15

Figure 1.5: Bearing (rolling) Friction ...................................................................... 17

Figure 1.6: Dahl Friction Model Example (Torque vs angle) (from [9]) ……………..... 18

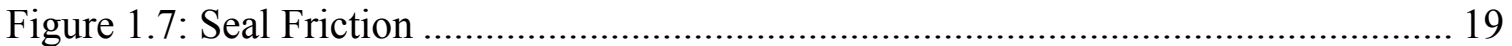

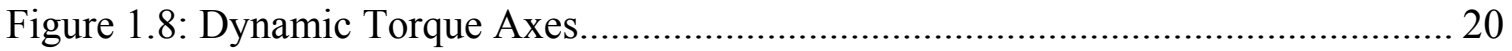

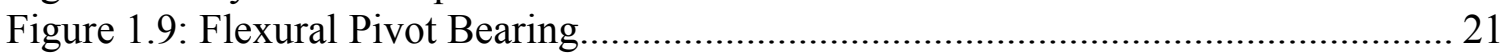

Figure 1.10: Example Structure Frequency Response (from [20])………………........ 23

Figure 1.11: Structural Dynamic Response including Structure Inertia (from [9]) .......... 24

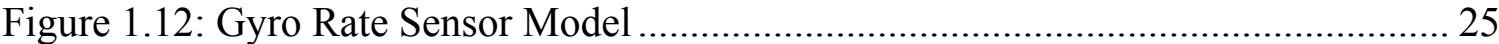

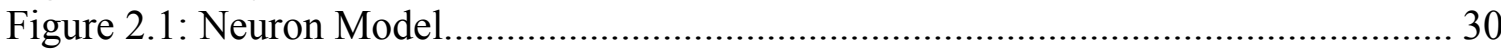

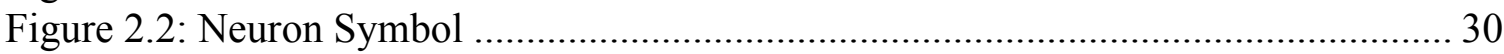

Figure 2.3: a) Linear Function b) Sigmoid Function c) Hyperbolic Tangent Function.... 31

Figure 2.4: Neural Network of Architecture 3-2-1 ...................................................... 33

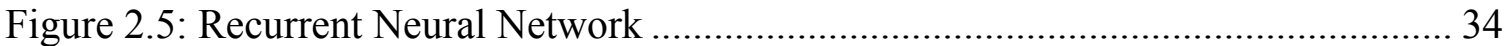

Figure 2.6: Focused Time Lagged Feedforward Neural Network.................................. 35

Figure 2.7: Block Diagram of Neural Network Training ………………......................... 37

Figure 2.8: Performance vs. Weights of Neural Networks ............................................... 40

Figure 2.9: Performance vs. Weight 2 ................................................................. 41

Figure 2.10: Performance vs. Weight 2 Given Weight 1............................................. 41

Figure 2.11: Output Neuron Signal Flow Graph ....................................................... 42

Figure 3.1: GUI for Time Domain Signal Creation Program ......................................... 58

Figure 3.2: Plant Response: $5 \mathrm{~Hz}$ Sine Wave (Section 3.3.1)........................................... 65

Figure 3.3: Plant Input: $35 \mathrm{~Hz}$ Sine Wave (first .5 seconds out of 12) ............................ 66

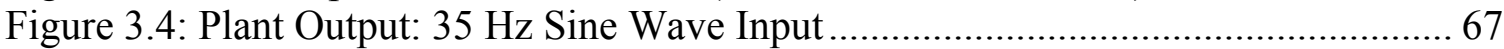

Figure 3.5: Plant Output: $35 \mathrm{~Hz}$ Sine Wave Input (first 0.5 seconds out of 12).............. 68

Figure 3.6: Plant Response: Multiple Frequency Wave (Section 3.3.2).......................... 69

Figure 3.7: Plant Response: Single Freq with Multiple Appended (Section 3.3.3)......... 70

Figure 3.8: Plant Output: Single Freq with Multiple Appended Input (Section 3.3.3).... 71

Figure 3.9: Single Freq with Multiple Appended Zoomed $(10 \mathrm{~Hz})$............................... 71

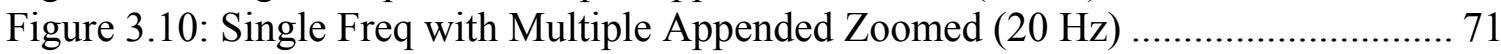

Figure 3.11: Single Freq with Multiple Appended Zoomed $(30 \mathrm{~Hz})$............................. 72

Figure 3.12: Single Freq with Multiple Appended Zoomed ( $50 \mathrm{~Hz} \&$ Multiple Freq) .... 72

Figure 3.13: Plant Response: Chirp Signal (Section 3.3.4) ........................................... 73

Figure 3.14: Chirp Zoomed: Low Freq.................................................................. 73

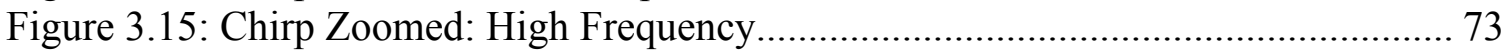

Figure 3.16: Chirp Response: Output at Low Freq.................................................. 74

Figure 3.17: Chirp Response: Output Zoomed ........................................................ 74

Figure 3.18: Plant Response: Random Signal (ARARI) (Section 3.3.5)......................... 75

Figure 3.19: Plant Response: Step Function (Section 3.3.6) ........................................ 77

Figure 4.1: Program for Training \& Simulating Neural Networks.................................. 79 
Figure 4.2: Training Setup for MATLAB Program.......................................................... 80

Figure 4.3: Network Structure (Standard Feedforward) …………………….................. 89

Figure 4.4: Performance Plot (Standard Feedforward NN) ........................................... 90

Figure 4.5: Performance Zoomed (Standard Feedforward NN) ....................................... 90

Figure 4.6: Simulation (Standard FF Structure) …………......................................... 91

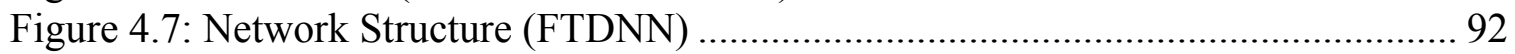

Figure 4.8: Performance (FTDNN Structure) ……………........................................... 93

Figure 4.9: Performance Zoomed (FTDNN Structure)................................................. 93

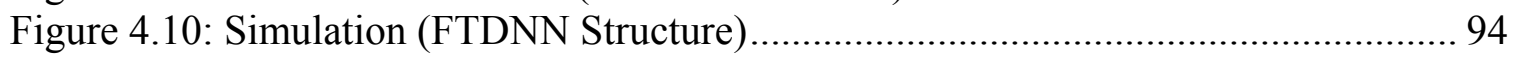

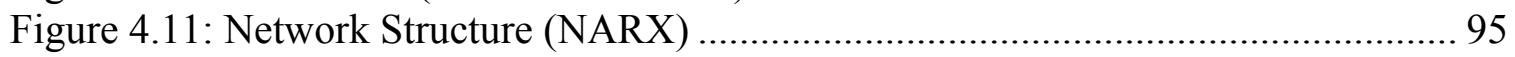

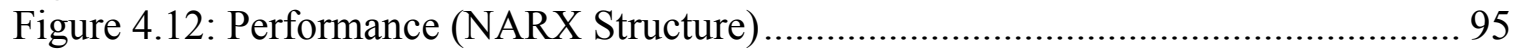

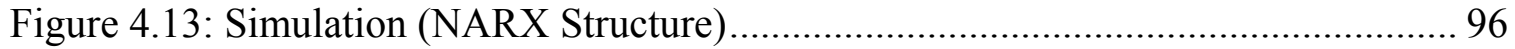

Figure 4.14: NARXSP Network Structure................................................................ 97

Figure 4.15: Performance (NARXSP w/ 5 neurons) …………………………........... 97

Figure 4.16: Performance (NARXSP w/ 5 neurons) Zoomed .......................................... 98

Figure 4.17: Simulation (NARXSP Structure w/ 5 neurons).......................................... 98

Figure 4.18: NARXSP Networks (3,2 layers with different delays) ................................ 99

Figure 4.19: 2 Neuron NARXSP Network ……………….................................... 102

Figure 4.20: Performance (Chirp Signal) .................................................................... 103

Figure 4.21: Performance (Chirp Signal) Zoomed …………......................................... 103

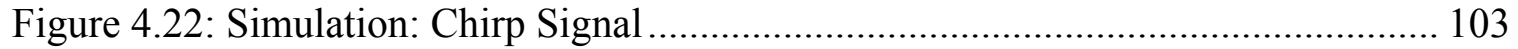

Figure 4.23: Simulation: Chirp Signal Zoomed (1st 0.1 seconds)................................. 104

Figure 4.24: Performance (Step Function)................................................................. 105

Figure 4.25: performance (Step Function) Zoomed ……………………………….... 105

Figure 4.26: Simulation: Step Input (Section 4.3.9) ………………........................... 105

Figure 4.27: Performance (Random Signal) ........................................................... 106

Figure 4.28: Performance (Random Signal) Zoomed................................................. 106

Figure 4.29: Simulation: Random Signal (Section 4.3.8) ............................................ 106

Figure 4.30: Performance (Mult Freq) ..................................................................... 107

Figure 4.31: Performance (Mult Freq) Zoomed ……………....................................... 107

Figure 4.32: Simulation: Multiple Frequency Wave (Section 4.3.4)............................. 108

Figure 4.33: Performance (Mult Single Freq w/ Mult)................................................. 109

Figure 4.34: Performance (Mult Single Freq w/ Mult) Zoomed ................................... 109

Figure 4.35: Simulation: Single \& Multiple Frequency Waves .................................... 109

Figure 4.36: Simulation: Single \& Multiple Frequency Waves Zoomed ........................ 110

Figure 4.37: Performance (35 Hz Sine Wave)........................................................... 111

Figure 4.38: Performance (35 Hz Sine Wave) Zoomed ............................................... 111

Figure 4.39: Simulation: $35 \mathrm{~Hz}$ Sine Wave ……………............................................ 111

Figure 4.40: Simulation: $35 \mathrm{~Hz}$ Sine Wave Zoomed ( $\left(1^{\text {st }} 0.35\right.$ seconds) ......................... 112

Figure 4.41: Frequency Spectrum for the Random Input Signal ................................... 115

Figure 4.42: Generalization Study: NARXSP Network …………………................... 116

Figure 4.43: Generalization Study - Performance (Random Signal) .............................. 117

Figure 4.44: Generalization Study - Performance (Random Signal) Zoomed................ 117

Figure 4.45: Generalization Study Simulation Results (Random Signal) …………....... 117

Figure 4.46: Simulation: Chirp Signal (Generalized Network) ..................................... 118

Figure 4.47: Simulation: Chirp Signal (Generalized Network) Zoomed......................... 119 
Figure 4.48: Simulation: Multiple Frequency Wave (Generalized Network) ............... 120

Figure 4.49: Simulation: $5 \mathrm{~Hz}$ Sine Wave (Generalized Network) ............................. 121

Figure 4.50: Simulation: $35 \mathrm{~Hz}$ Sine (Generalized Network).................................... 122

Figure 4.51: Simulation: $35 \mathrm{~Hz}$ Sine (Generalized Network) Zoomed ......................... 123

Figure 4.52: Simulation: Single \& Multiple Freq Waves (Generalized Network) ......... 124

Figure 4.53: Simulation: Single \& Mult Freq Waves (Generalized Network) Zoomed. 125

Figure 4.54: Simulation: Step Function (Generalized Network) ................................ 126

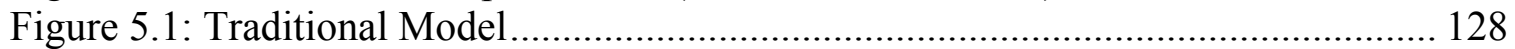

Figure 5.2: Inner Azimuth Gimbal (Rigid Structure) ............................................... 129

Figure 5.3: Gimbal (Rigid Structure w/ Spring \& Dampening Torque Disturbances)... 131

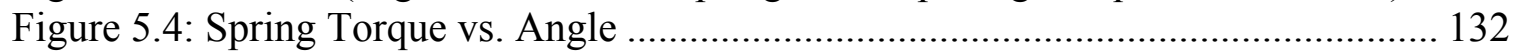

Figure 5.5: Angular displacement vs. Time for a .5 Volt Step Input........................... 133

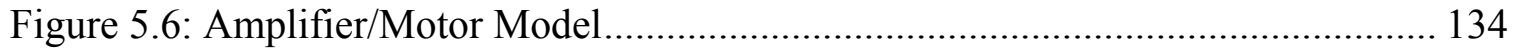

Figure 5.7: Gyro Rate Sensor............................................................................... 135

Figure 5.8: Measured Gyro Rate Sensor Noise ........................................................ 136

Figure 5.9: Measured Gyro Rate Sensor Noise (Zoomed) ........................................ 137

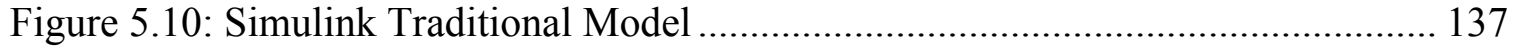

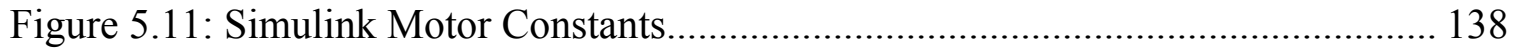

Figure 5.12: Simulink Amplifier and Motor Model .................................................. 139

Figure 5.13: LTI Model Simulation Results (Random Signal) ................................... 140

Figure 5.14: LTI Model Simulation Results (Chirp Signal) ...................................... 141

Figure 5.15: LTI Model Simulation Results (Multiple Frequency)............................... 142

Figure 5.16: LTI Model Simulation Results (5 Hz Sine Wave) .................................. 143

Figure 5.17: LTI Model Simulation Results (35 Hz Sine Wave) ................................ 144

Figure 5.18: LTI Model Simulation Results (35 Hz Sine Wave) - Zoomed................... 145

Figure 5.19: LTI Model Simulation Results (Single \& Multple Frequency) ................. 146

Figure 5.20: LTI Model Simulation Results (Single \& Multple Frequency) - Zoomed. 147

Figure 5.21: LTI Model Simulation Results (Step Function) ...................................... 148

Figure 5.22: Traditional vs. NN Model Outputs: Random Input Signal ...................... 149

Figure 5.23: Traditional vs. NN Model Outputs: Chirp Input Signal ........................... 150

Figure 5.24: Traditional vs. NN Model Outputs: Chirp Input (Zoomed) ...................... 151

Figure 5.25: Traditional vs. NN Model Outputs: Multiple Freq Input Signal ................ 152

Figure 5.26: Traditional vs. NN Model Outputs: 5 Hz Sine Input Signal ..................... 153

Figure 5.27: Traditional vs. NN Model Outputs: 5 Hz Sine Input (Zoomed) ................ 154

Figure 5.28: Traditional vs. NN Model Outputs: 35 Hz Sine Input Signal ................... 155

Figure 5.29: Traditional vs. NN Model Outputs: 35 Hz Sine (Zoomed 1) .................... 156

Figure 5.30: Traditional vs. NN Model Outputs: 35 Hz Sine (Zoomed 2) .................... 157

Figure 5.31: Traditional vs. NN Model Outputs: Single \& Multiple Freq Input Signal. 158

Figure 5.32: Traditional vs. NN Model Outputs: Single \& Mult. Freq (Zoomed) ......... 159

Figure 5.33: Traditional vs. NN Model Outputs: Step Function Input Signal................ 160

Figure 5.34:Traditional vs. NN Model Outputs: Step Function Input (Zoomed) ........... 161 


\section{Chapter 1: Introduction}

Video camera systems have become an integral part of a growing global information market. Frequently, camera systems are placed on helicopters and other vehicles that are utilized by the military, law enforcement and for various consumer applications such as television news programs. One of the most desirable characteristics for these applications is to view objects at great distances through the camera without any vibration or "shaking" of the image or picture. As image stability increases, so does the distance at which objects can be viewed.

The camera systems consist of a gimbal unit that houses a camera. This gimbal is typically mounted to some type of aircraft, but is often placed on boats or land vehicles. The photograph of a camera system primarily used on helicopters and built by Axsys Technologies is depicted in Figure 1.1 below.

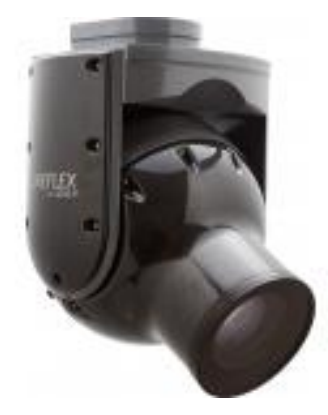

Figure 1.1: Gyro-stabilized Camera System

Currently, these camera systems are stabilized along their line of site (LOS) using a linear closed-loop control strategy. This stabilizes against unwanted angular movements. 
The camera is often mounted in a roll tube that is contained within a two-axis gimbal. This "inner" unit is, in turn, often mounted inside another two-axis "outer" gimbal. A representation of a two-axis gimbal is shown in Figure 1.2 below.

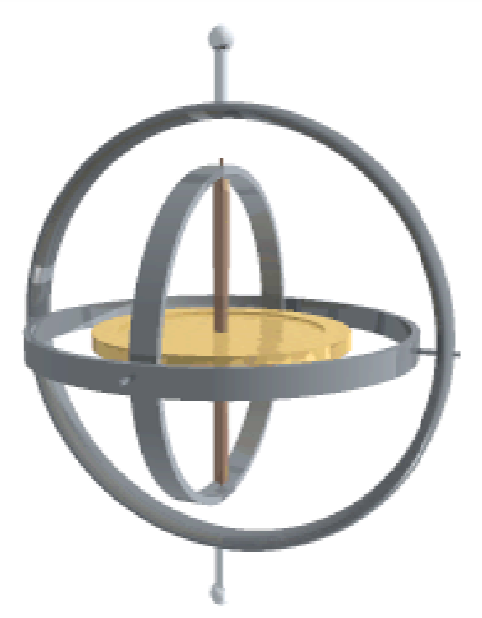

Figure 1.2: Two-Axis Gimbal

The outer two-axis gimbal allows the camera to be panned 360 degrees in azimuth and over 180 degrees in elevation. The inner two-axis gimbal has a limited range of movement (a few degrees of travel), but has a much larger bandwidth than the outer gimbal. This higher bandwidth gives the inner gimbal the ability to counteract the smaller amplitude, higher frequency vibrations of the mounting surface (such as a helicopter). Two general categories of performance requirements usually dominate the control system design trade-offs. These trade-offs are: (1) the LOS (line of sight) stability or "jitter" must be held within bounds, usually specified in miroradians (or fractions of a microradian) and (2) the LOS must faithfully follow input commands [10]. 
An accurate model of the system must be created in order to control the system. . In this thesis a model for the inner gimbal azimuth axis is created using a neural network. Currently, these systems are often modeled as linear. The block diagram in Figure 1.3 below shows a traditional model of one axis of the inner gimbal [9].

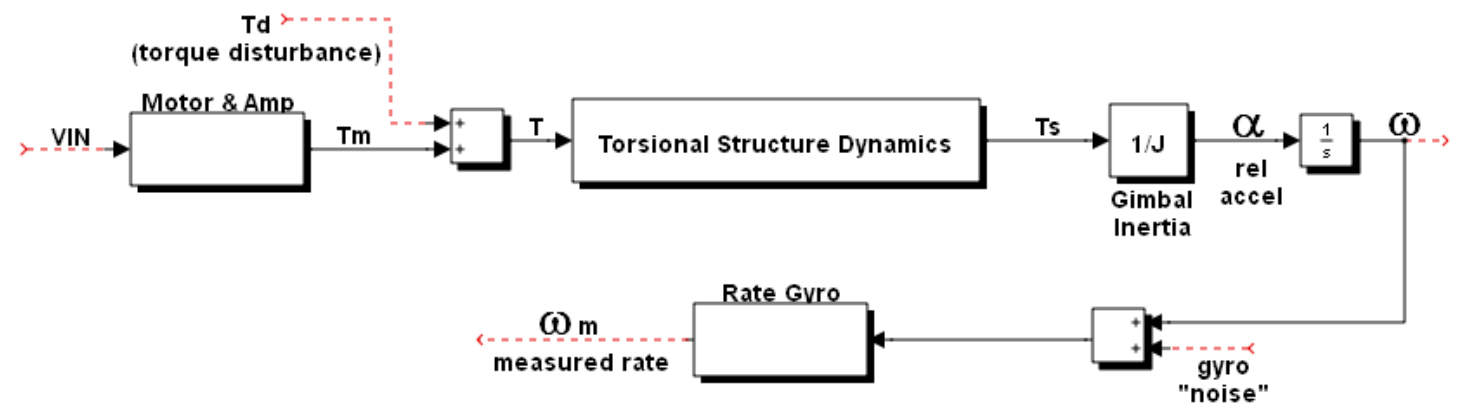

Figure 1.3: Model: Inner Gimbal Azimuth Axis

The input to the system is a voltage VIN and the output is relative (between inner and outer gimbal) measured angular rate $\omega_{m}$ in radians/s. The outer gimbal is assumed to be stationary in inertial space

The input voltage VIN drives the input of an amplifier and the output of the amplifier in turn drives a "torquer" motor consisting of a coil and magnet. The coil is attached to the outer gimbal, the magnet is attached to the inner gimbal.

This topology limits the inner gimbal capability to a few degrees of angular movement. The torque $\mathrm{T}$ (as shown in the figure above) acts on the inner gimbal azimuth axis structure. The torque $\mathrm{T}$ consists of the addition of motor torque $T_{M}$ and torque disturbances $T_{d}$.

The structure acceleration due to this torque is a function of the dynamics of the structure and the reciprocal of the structures moment of inertia (inertia tensor) $\mathrm{J}$ [10]. The 
inertial angular rate $\omega$ is measured using a gyro rate sensor attached to the structure. This rate $\omega$, and thus the measured rate $\omega_{m}$, are relative between the inner and outer gimbal.This assumes that the outer gimbal is stationary in inertial space. Thus, $\omega_{m}$ is equal to the system output (measured relative angular rate).

A block diagram of the amplifier and motor combination is shown in Figure 1.4 below.

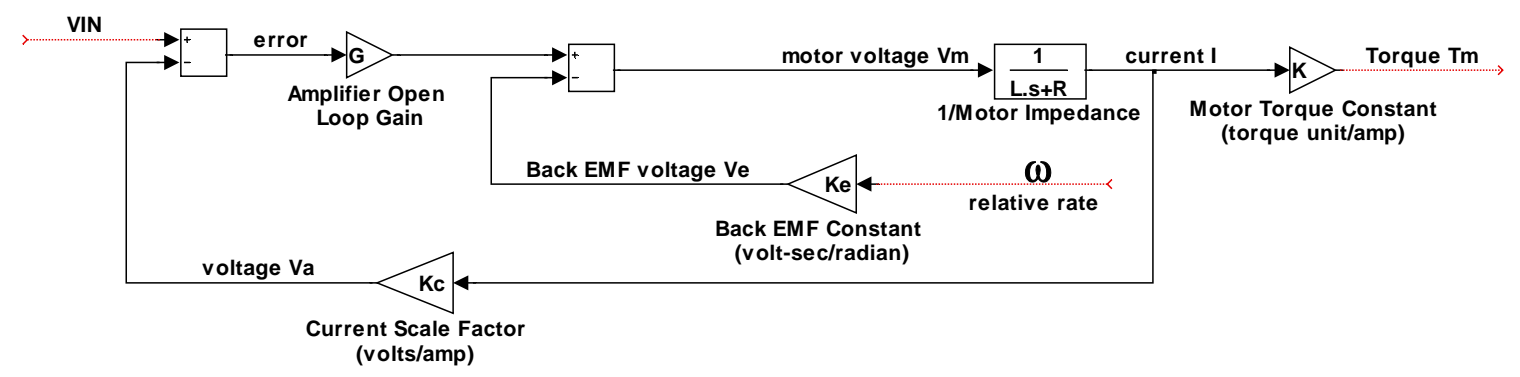

Figure 1.4: Motor and Amplifier Block Diagram

The above figure consists of a constant current amplifier with a nominal gain of one and the torquer motor described previously. The amplifier closes a loop around the motor current. This amplifier consists of open loop gain, G, the measurement of motor current, I, the conversion of this current to voltage, $V_{a}$ (set by $\left.K_{c}\left(\frac{\text { volts }}{a m p}\right)\right)$ and the calculation of the error between the desired and actual current (VIN $\left.-V_{a}\right)$.

The model for the motor consists of the motor impedance $L s+R(\mathrm{~L}$ is the inductance of the motor and $\mathrm{R}$ is the resistance), the Back EMF constant of the motor $K_{e}$ (units of $\frac{\text { volts }}{\frac{r a d}{s e c}}$ or $\frac{\text { volt-sec }}{\text { rad }}$ ), and the motor torque constant $\mathrm{K}$ (units of $\frac{l b s-i n}{a m p}$ ). 
By referencing the above figure the motor torque $T_{m}$ can be written as $T_{m}=$ $\left(\frac{G}{L S+R+K_{c} G}\right) K V I N-\left(\frac{K K_{e}}{L S+R+K_{c} G}\right) \omega$. Note that if $K_{c}=1$ and $\mathrm{G}$ is very large then the motor torque $T_{m}$ approximatly equals the system input voltage VIN multiplied by the motor torque constant $\mathrm{K}\left(T_{m} \approx K \times \operatorname{VIN}(l b s-i n)\right)$.

Referencing Figure 1.3 again: the motor torque $T_{m}$ is combined with the disturbance torque $T_{d}$ to create the total torque $\mathrm{T}$. This torque is applied to the structure. The acceleration of the structure is a function of the total torque $\mathrm{T}$, the dynamics of the structure, and the structure moment of inertia J. The acceleration of the structure is derived by dividing torque $T_{S}$ (a result of the structure dynamics) by the moment of inertia J. This calculation can be explained using Newton's Law: $T=M_{a} A_{a}=J \frac{d \dot{\theta}}{d t}=J \ddot{\theta}$ where $M_{a}$ is the mass of the inner gimbal structure, $A_{a}$ is the angular acceleration of the inner gimbal relative to the outer gimbal, and $\theta$ is the angle of the inner gimbal relative to the outer. The rate can be calculated from this torque as $\omega=\dot{\theta}=\int \ddot{\theta}=\int \frac{T}{J} d t$. This calculation assumes the gimbal is a rigid body, a homogeneous mass (unless the full inertia tensor is used), and single axis rotation (unless rates and accelerations are vectorized) [9].

The torque disturbance $T_{d}$ (shown in Figure 1.3) for an inner gimbal can include friction, unbalance of the camera/payload, static and dynamic unbalance, gyroscopic torques (such as from the gyro rate sensor), on-board shaking forces, actuator and gear reactions, cable torques, spring torques, structural bending, actuator cogging, and ripple torques [9]. These torques may or may not be present depending upon the specific design 
of the gimbal system. Each of these torque disturbances are described in more detail below.

The torque disturbance caused by friction in a gimbal system usually consists of sliding or rubbing friction from the seals and bearing friction.

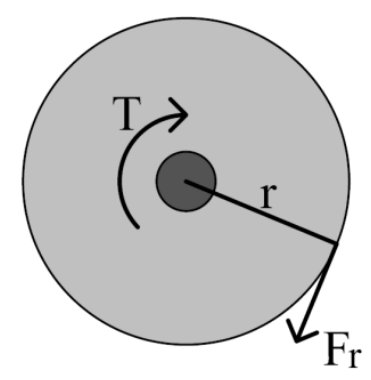

Figure 1.5: Bearing (rolling) Friction

The torque T (shown in Figure 1.5) due to bearing (rolling) "static" friction can be modeled using the following formula: $T=\mu_{\mathrm{a}} \mathrm{F}_{\mathrm{a}}+\mu_{\mathrm{r}} \mathrm{F}_{\mathrm{r}}$. In this equation, $\mathrm{r}=$ mean bearing radius (in.), $F_{a}=$ axial load including preload (lbf), $F_{r}=$ radial load (lbf), $\mu_{\mathrm{a}}=$ coefficient of rolling friction, and $\mu_{\mathrm{r}}=$ coefficient of rolling friction.

One of the characteristics of the friction present in inertial stabilization is a nonlinear phenomena called Coulomb friction [11]. The Coulomb model describes friction force $F_{c}=\mu_{c} F_{N} \operatorname{sgn}(v)$ where $\mu_{c}$ is the Coulomb friction coefficient, $F_{N}$ is the normal force between the two objects, and $v$ is the velocity between the two objects.

One friction model used for ball bearings is the Dahl model [17]. In 1968, P.R. Dahl proposed an alternative to the Coulomb friction model. While observing the behavior of ball bearings, he noted that input forces with very small amplitude were reacted against by small elastic restoring forces [20]. Dahl compares the transisition from static to kinetic friction to that of elastic to plastic deformation in ductile materials. His initial observation of non-linear friction effects occurred while observing ball bearings 
acted on by low amplitude input forces. The elasticity observed results in hysteresis; at loads below $F_{c}$ (sliding Coulomb friction). This force can be likened to torque $T_{s}$ in the figure below for bearings [9]. Figure 1.6 demonstrates the Dahl observation between bearing torque and angle.

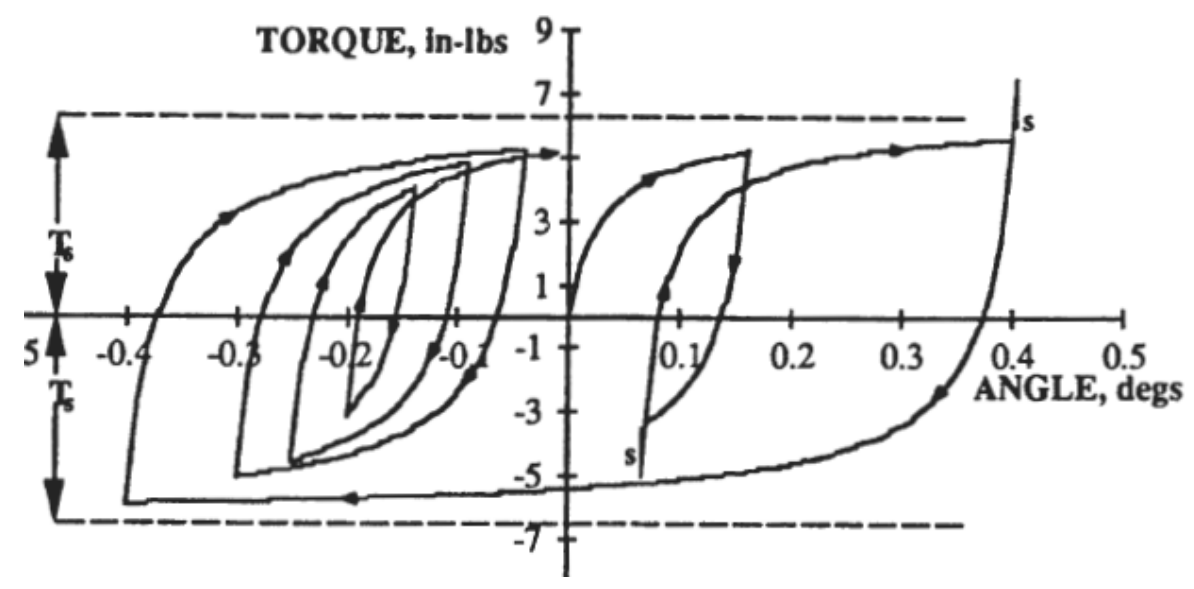

Figure 1.6: Dahl Friction Model Example (Torque vs angle) (from [9])

The Dahl model for a bearing defines the torque rate as $\frac{\mathrm{d} T_{\mathrm{B}}}{\mathrm{d} \theta}=\gamma\left[\mathrm{T}_{\mathrm{S}}-T_{\mathrm{B}} \times \operatorname{sgn}(\dot{\theta})\right]^{\beta}$ where $\gamma=\frac{\mathrm{s}}{\mathrm{T}_{\mathrm{S}}+\left|\mathrm{T}_{\mathrm{P}}\right|}, T_{B}$ is the torque on the bearing (y-axis in Figure 1.6 above), $\theta$ is the bearing angle (x-axis in above figure), $\mathrm{s}=$ slope at reversal (reversal of torque $T_{B}$ direction), $T_{s}$ is the maximum Dahl friction torque, $T_{p}$ is the value of torque at turnaround or reversal and $\beta$ (values range $\geq 0$ ) is the parameter that determines the shape of the stress-strain curve (Torque vs angle curve) [17]. Some gimbals incorporate seals for EMI and environmental reasons. The disturbance torque resulting from these seals can be modeled as a sliding or rubbing friction. 


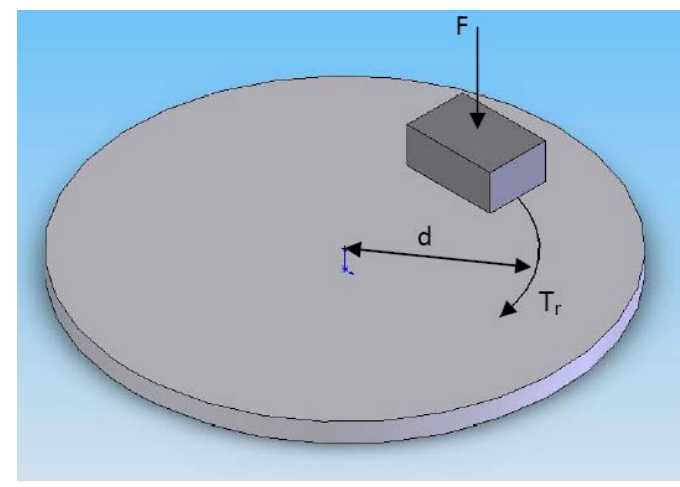

Figure 1.7: Seal Friction

This torque (reference Figure 1.7 above) is defined as $T_{r}=\mu F d$ where $\mathrm{d}$ is the perpendicular distance between the line-of-action of $\mathrm{F}$ and the rotating axis (in), $\mathrm{F}$ is the force holding the two surfaces together (lbF), and $\mu$ is the coefficient of friction between the two surfaces. Seals between the inner gimbal and outer gimbal can sometimes contain fluid. Thus, viscous friction might have to be modeled. The torque due to this type of friction can be defined as $T=k_{v} \omega$ where $k_{v}$ is the coefficient of viscous friction $\left(\frac{i n \cdot l b f \cdot s e c}{r a d}\right)$ and $\omega$ is the relative angular velocity $\left(\frac{r a d}{s}\right)$.

The torque disturbance caused by the balance of the payload within the inner gimbal has an effect on the system dynamics. Static unbalance can cause a disturbance torque which can be modeled as $T_{\mathrm{u}}=\varepsilon \mathrm{Wg}$ where $\varepsilon$ equals the center of gravity offset, $\mathrm{W}$ is the weight of the rotating assembly, and $\mathrm{g}$ is the gravity plus acceleration vector perpendicular to the rotating axis. 


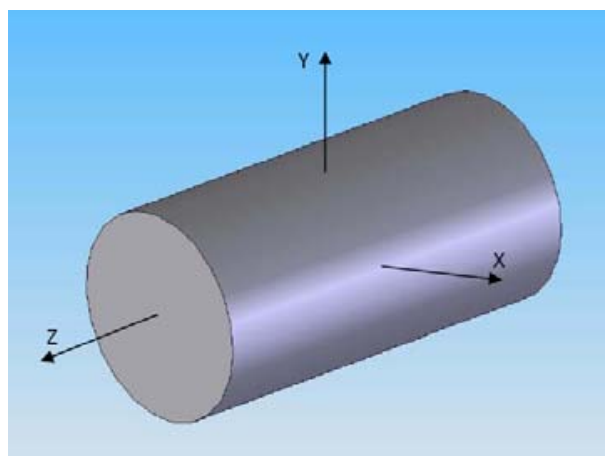

Figure 1.8: Dynamic Torque Axes

Dynamic unbalances are caused by a non-symmetrical distribution of mass. This torque can be modeled using the formula: $T_{Z}=I_{X Y} \omega_{X}{ }^{2}$. In this equation, $I_{X Y}$ is the product of inertia for the $\mathrm{x}$ and $\mathrm{y}$ axes shown in Figure 1.8 above (in-lbf- $\mathrm{s}^{2}$ ) and $\omega_{x}$ is the angular velocity about the $\mathrm{x}$-axis $\left(\frac{\mathrm{rad}}{\mathrm{s}}\right)$.

Aniso-elastic unbalance is caused by un-symmetrical elasticity,and requires vibration in 2 dimensions simultaneously. It is difficult to predict, and may require FEA analysis [9]. This torque can be defined using the equation $T_{a e}=K w g_{x} g_{y}$ where $\mathrm{K}$ is the aniso-elatic constant for a particular mechanism $\left(\frac{i n}{g^{2}}\right), \mathrm{W}$ is the weight of the rotating assembly (lbf), and $\mathrm{g}$ is the gravity plus acceleration vector perpendicular to the rotating axis (gs).

Gyroscopic "gyro" torque (precession \& nutation) is a torque disturbance caused by the fact that in many cases the rate is measured using a sensor utilizing a spinning mass. This causes a torque on the object being measured. A gyro torque can be defined as $T_{X}=I_{Z} \omega_{Z} \omega_{Y}$ where $I_{Z}$ is the inertia about the $\mathrm{z}$ axis (in-lbf-s $\left.{ }^{2}\right), \omega_{Z}$ is the angular velocity about the $\mathrm{z}$ axis, and $\omega_{y}$ is the angular velocity about the y-axis. 
Disturbance torques can also be caused by shaking forces on the payload (camera). For example, a shaking force could be caused by a cryogenic cooler used with an infrared camera. The torque caused by a shaking force is defined as $T_{s f}=F_{s} d$ where $\mathrm{d}$ is the perpendicular distance from the shaking force to center of rotation and $F_{S}$ is the force.

Spring torques are another form of disturbance torque and can be caused by cable wraps or Flexural Pivot bearings (a type of spring). Cables are modeled as a spring; however, they are actually quasi-linear and have a dampening effect [9]. Flexural Pivot bearings have a limited range of angular movement but are sometimes used instead of ball bearings to get rid of the non-linear effects of this type of bearing. A picture of a Flexural Pivot bearing is shown in the figure below.
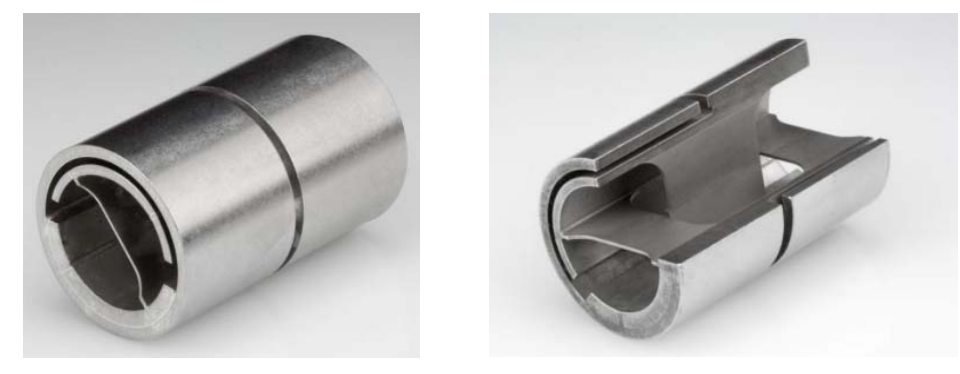

Figure 1.9: Flexural Pivot Bearing

A spring torque is defined as $T_{s p}=K_{s} \Delta \theta$ where $K_{s}$ is the spring coefficient for a particular mechanism $\left(\frac{i n-l b f}{r a d}\right)$ and $\Delta \theta$ is the relative rotation measured from point of zero torque (rad). 
Structural bending disturbance torques result from base vibrations that may or may not be sensed by the gyro [10]. Structural flexure can also cause torque disturbances and can be modeled using Finite Element Analysis [9]. For example, a structural flexure includes bending, optical component displacements and Aniso-elastic effects [9].

The total torque $\mathrm{T}$ (as seen in Figure 1.3) is a combination of one or more of the torque disturbances listed previously and the motor torque. This total torque is applied to the inner azimuth gimbal structure. To model the relationship between this torque and the acceleration of the structure both the structure dynamics and inertia must be taken into account. Although practical systems are multiple degree of freedom (MDOF) and have some degree of nonlinearity, their structure dynamics can generally be represented as a superposition of single degree of freedom (SDOF) linear models [19].

The system of interest in this paper is the inner gimbal azimuth axis. system The dynamics for this system include the torsional interaction between the total torque $\mathrm{T}$ and the gyro rate sensor. The transfer function for this relationship is typically derived from FEA (finite element analysis) or modal analysis using the first four to five modes [9]. The response at one point in the structure to a force or torque excitation at another point in the structure can be defined as:

$$
\underset{\begin{array}{c}
\text { Response } \\
\text { at node } j
\end{array}}{U_{j}}=\sum_{i=1}^{n} \frac{\varphi_{i j} \times \varphi_{i k} / M_{i}}{\underbrace{s^{2}+2 \delta_{i} \omega_{i} s+\omega_{i}^{2}}_{\begin{array}{c}
\text { Transfer Function } \\
\text { (ith mode component) }
\end{array}}} \times \underbrace{f_{k}}_{\begin{array}{c}
\text { Force or Torque } \\
\text { input at node } k
\end{array}}
$$

The following parameters from the above equation are available from FEA analysis: $\varphi_{i j}=j$ is the eigenvector term of the response degree of freedom in the ith mode, $\varphi_{i k}=k$ is the eigenvector term of the input degree of freedom in the ith mode, $M_{i}=$ Generalized mass in the ith mode, $\omega_{i}=$ Natural frequency (eigenvalue) of the ith 
mode, $\delta_{i}=$ Damping ratio of the ith mode (typically .025 to .05 for metallic structure).

The inputs to and outputs from the above equation are: $f_{k}=$ Excitation force (or torque) at the kth degree of freedom, $U_{j}=$ Response displacement (either translational or rotational) at the jth degree of freedom [9]. An example of a typical frequency response plot and the individual contribution from each mode is shown in the figure below.
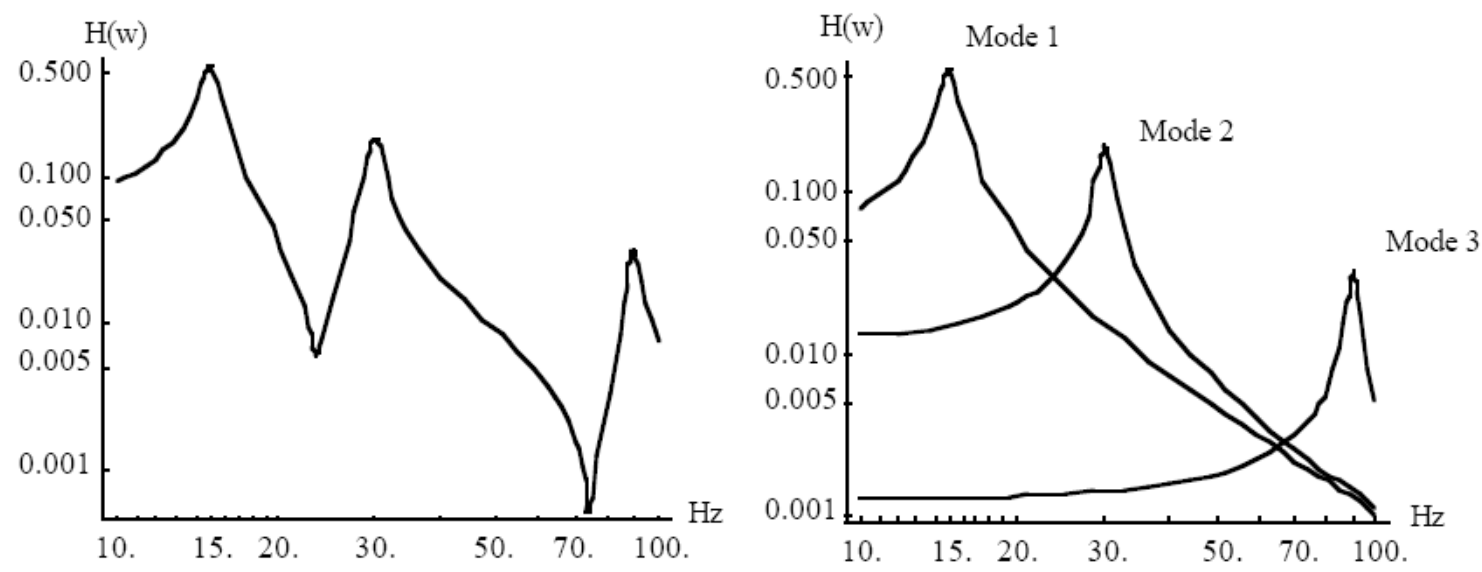

Figure 1.10: Example Structure Frequency Response (from [20])

The torsional structural dynamics combined with the rigid body inertia $\mathrm{J}$ (see Figure 1.3) will give a response typical of the example shown below [9]. 


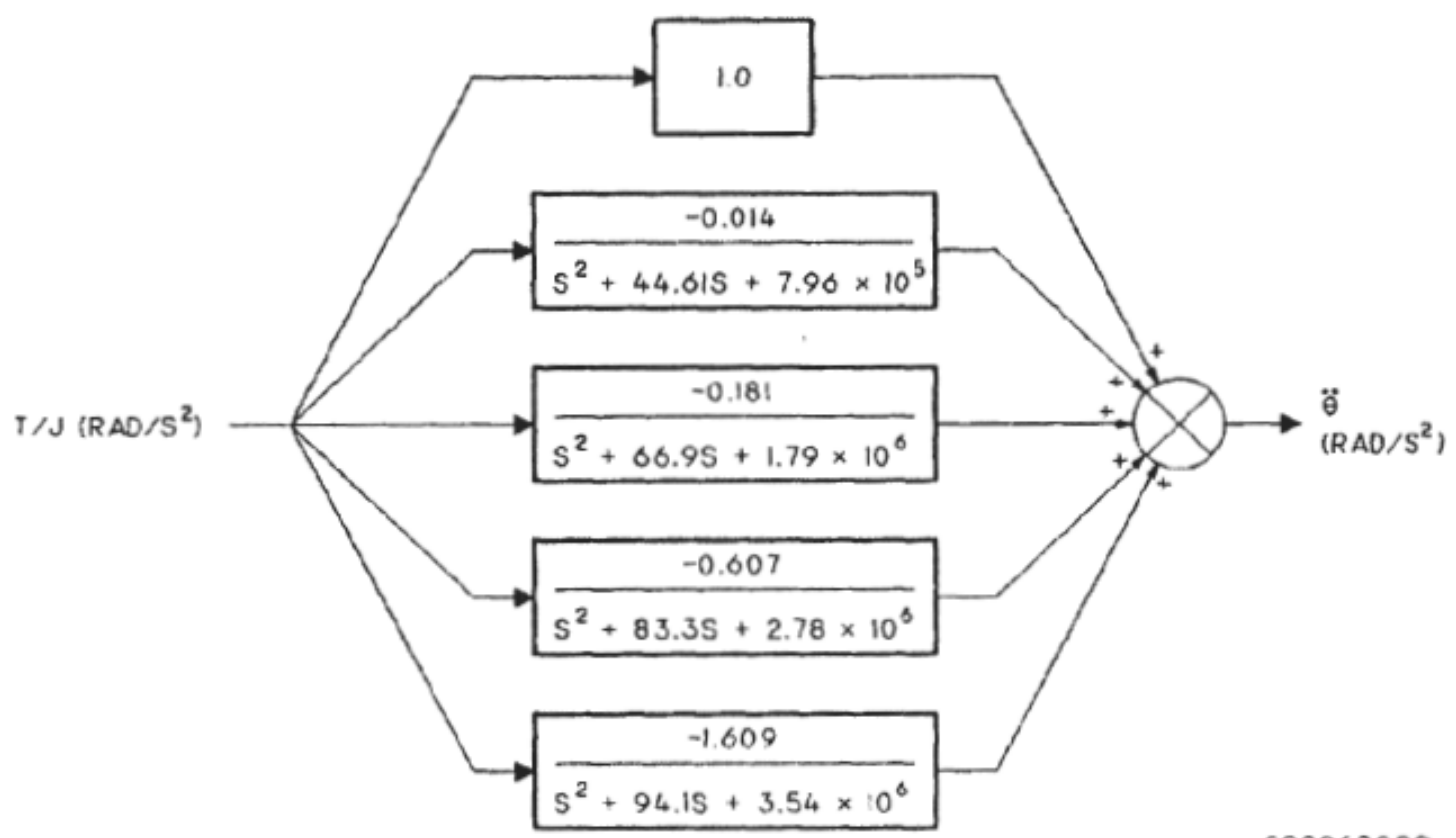

C98268089

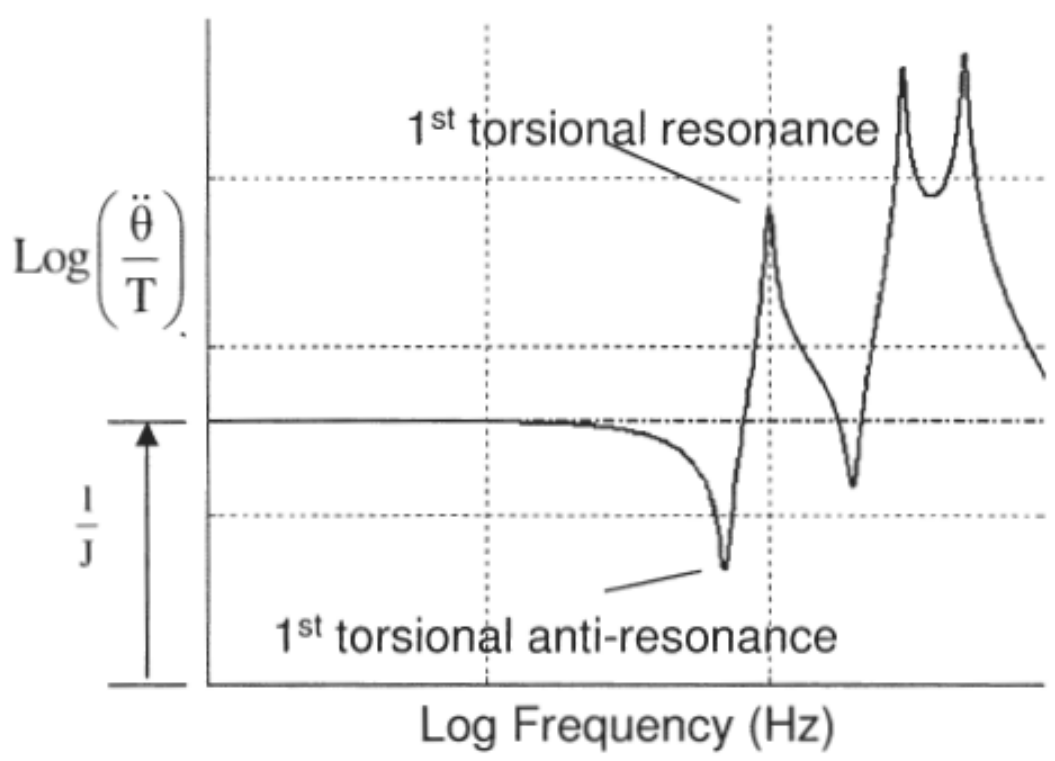

Figure 1.11: Structural Dynamic Response including Structure Inertia (from [9])

It is important to note from the above figure that at DC and low frequencies the frequency response of the structure is equal to the reciprocal of the structures moment of inertia about the axis being controlled. Also note that each of the second order equations in the figures block diagram represents a structural mode. 
The gyro rate sensor as shown in Figure 1.3 above can be modeled as a second order low pass filter with random noise added to input [9]. This model with input $\omega$ (angular rate) and output $\omega_{m}$ (measured angular rate) is shown in Figure 1.12 below.

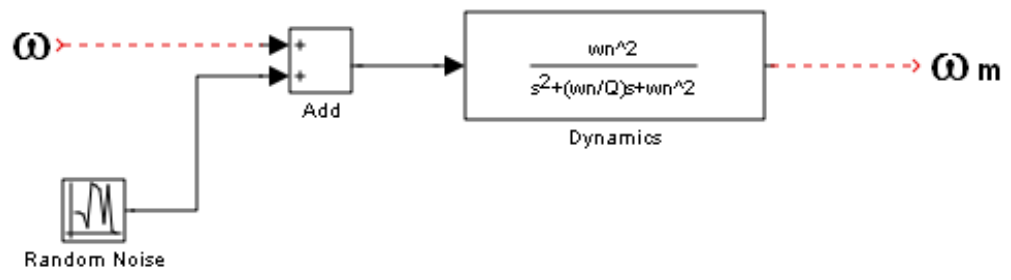

Figure 1.12: Gyro Rate Sensor Model

In some systems, modeling of certain system dynamics is not accomplished and notch filters are added after testing to get rid of undesirable frequencies [12]. Such undesirable frequencies are common in many aerospace flexible dynamic systems where the desired center frequency shifts due to the nonlinearities and coupling of the system. An approach to eliminate these frequencies is to place notch filters after taking systems measurements. This requires significant verification and validation activity as well as a large storage for the filter coefficients.

A modeling strategy using neural networks is worth investigating due to the limitations of traditional approaches. A neural network can compute nonlinear relationships, which allows it to be trained to model an unknown system. It can generalize (map similar inputs to similar outputs)and is robust in the presence of noise. A neural network has the ability to be made adaptive by training online. Due to these characteristics, a neural network has inherent advantages over traditional approaches for modeling. 
As mentioned earlier, gyro-stabilized gimbal systems include nonlinearities and are very complex due to complex structural dynamics and the many torque disturbances present. In multi-gimbaled systems there are un-modeled dynamics, kinematics of the gimbal plant which result from non-symmetry in the mechanical design including products-of-inertia, gyroscopic effects, and kinematic coupling [10].

The system identified in this paper is the inner azimuth gimbal of a multigimbaled system and is thus exposed to the characteristics and problems listed above. Specifically, the neural network model includes torque disturbances and nonlinearities from bearing friction Coulomb model effects, kinematic coupling effects, and cable nonlinearities. Aniso-elastic or dynamic unbalance. Dynamic unbalance is difficult to predict and may require FEA analysis.

Another advantage to modeling an inner gimbal system (as represented in Figure 1.3) using neural networks is to utilize this model in an adaptive control strategy. Strategies such as model reference control, predictive control, and feedback linearization control use plant models. These strategies could be made adaptive by using a neural network model and controller. Few stabilized gimbal system (such as the system being identified in this paper) parameters are stationary. The dynamic operating environment, for example, is usually undergoing constant change and only a few typical or worst case scenarios can be investigated during the design state (assuming the environment and the plant can be accurately characterized) [10]. In addition to the above, generalization and robustness to noise are also problems because of the complexity of these systems. It is possible that a neural network model could solve some of these problems. 
In this thesis, an artificial neural network model is created for system identification. The system to be identified is defined by the inertial response of the azimuth axis of the inner gimbal.

The model is created by training the network to map the relationship between system input (voltage VIN in Figure 1.3) to system output (measured relative angular rate $\omega_{m}$ in Figure 1.3). The network is trained using experimentally measured data from the gyro-stabilized camera system to be identified. (The camera system is built by Axsys Technologies in Grass Valley, California).

The model is created using several different types of time domain data input/output sets gathered from the real system. Several network structures are compared and the neural networks ability to generalize well over the full input range of the system is tested. This is accomplished by training the network on one data set and then simulating the networks response to several other inputs. The network response from these inputs is then compared to the actual system output. In addition, a traditional model is created and compared to the neural network model. This was accomplished by comparing the errors between the two model outputs with the real system output.

This thesis is organized into six chapters. An overview and theory of neural networks is presented in Chapter 2. The process of acquiring the data from the system to be modeled is described in Chapter 3. The simulation results for modeling the system using an artificial network is presented in Chapter 4. Chapter 5 includes a comparison between a traditional model and the neural network model. Finally, the summary and conclusion of this study are presented in Chapter 6, along with future works involving neural networks. 


\section{Chapter 2: Overview of Artificial Neural Networks}

The human biological system of neurons and synapses has inspired the development of artificial neural network metholdologies in computer science. In the human brain there are billions of neurons and synaptic connections that create a complex network that modifies itself as it learns through experience. Like the human brain, an artificial neural network can learn from experience and relationships.

As implemented in a computer system an artificial neural network learns by classifying data and recognizing patterns. It can then be trained to find solutions and forecast future events.

Artificial neural networks are commonly referred to as "neural networks". They are a method of computing input/output relationships comprised of a set of parallel processing neurons with interconnections called synapses. A network can have any number of inputs and outputs. Based on its inputs and an activation or transfer function, each neuron produces one output. A neuron is not limited to linear relationships. A value is placed on the output of a neuron which adjusts the importance, or weight, of a synapse. The result of this weighted synapse can be fed into the input of one or more other neurons or to the output of the network. These weights can be modified to adjust the outcome of the network.

\section{1 - Brief History of Neural Networks}

The concept of neural networks came about in 1943 when W.S. McCulloch and W.A. Pitts combined many simple units in order to design Boolean logic. Each unit 
performed a certain logic function. The units combined to produce the desired network output. Their network is generally considered to be the first artificial neural network. In 1949, Donald Hebb, a psychologist at McGill University, introduced an idea of learning when he published a biologically plausible learning rule. Reinforcement learning was introduced by Minsky around 1954.

In 1958, Frank Rosenblatt coined the term "perceptron", defined as a single layer network in which the neuron activation functions have a hard-limiting threshold.

In 1960, Bernard Widrow and Marcian Hoff at Stanford University created an algorithm called the least mean square (LMS) algorithm, also known as the Widrow-Hoff rule. This rule was applied to a simple adaptive linear neuron (ADALINE). The LMS algorithm was successfully used to classify linearly separable patterns.

Waltz and Fu independently introduced and applied reinforcement learning in control theory in 1965 and Michie and Chambers reinforced this in 1968.

In the 1970's, a large amount of attention was focused on artificial intelligence (AI). During this time several pioneering works were created, one of which did forecasting analysis using the concept of backpropagation (BP) proposed by Werbos in 1974.

Neural networks became more popular in the 1980's due to faster and cheaper computers and independent researchers advancing the field with new training algorithms. The backpropagation algorithm became popular in the mid 1980's and has led the field forward ever since. 


\section{2 - Neural Network Architecture}

A neural network consists of neurons, which are connected together to form a network. These neurons are generally grouped into layers. Each layer's outputs are fed into the next layer's inputs. The inputs to the network are fed into the first layer of neurons and the last layer of neurons provide the outputs of the network.

The neuron is an information-processing unit. Each neuron can have any number of inputs and one output. The unit consists of two basic components, the summer and the activation function (also called transfer function). A model of the neuron and its symbol are shown in Figure 2.1 and Figure 2.2 below.

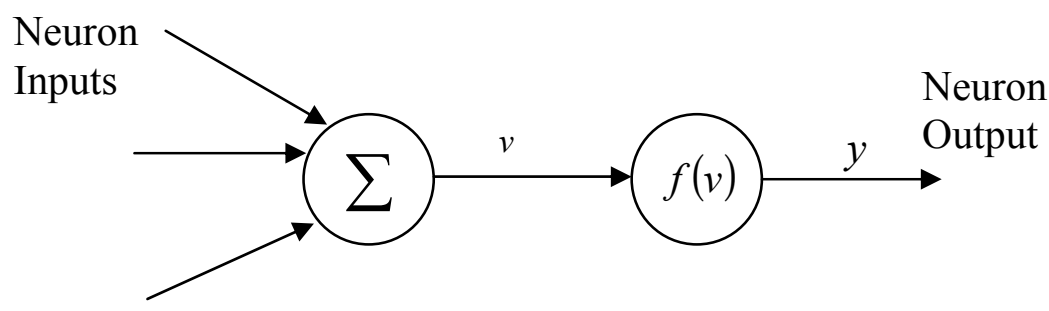

Figure 2.1: Neuron Model

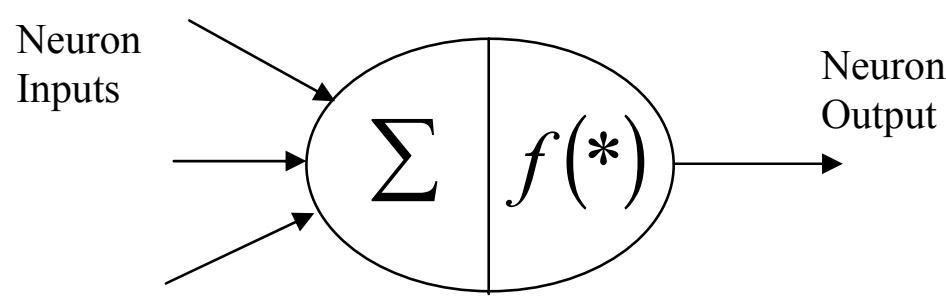

Figure 2.2: Neuron Symbol

As expected, the summer sums the inputs to the neuron. The result is passed through the activation function, which supplies the output of the neuron. Some common 
activation functions include the linear activation function, the sigmoid function, and the hyperbolic tangent function. Figure 2.3 below shows a graphical representation of the three activation functions.

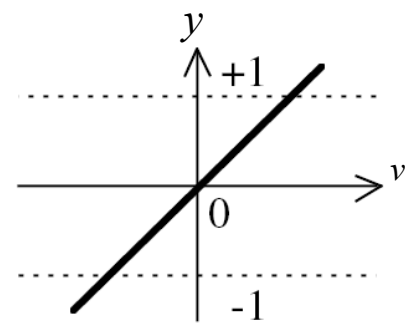

(a)

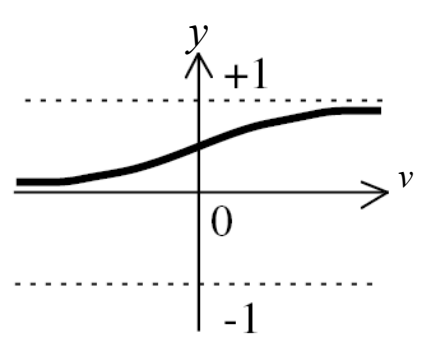

(b)

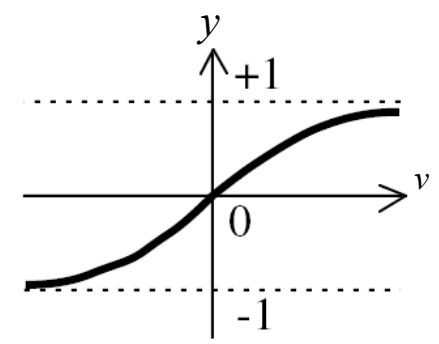

(c)

Figure 2.3: a) Linear Function b) Sigmoid Function c) Hyperbolic Tangent Function

The linear function has a slope of 1 and merely passes the input through the neuron. The sigmoid and hyperbolic tangent functions are limiting functions and are sometimes referred to as squashing functions for this reason. They accept an input of any range and produce an output in a strictly limited range, hence a squashing effect. The sigmoid 
functions output is limited between 0 and 1. The sigmoid function is defined in Equation 1 below.

$$
f(v)=\frac{1}{1+e^{-v}}
$$

The hyperbolic tangent functions output is limited in range between -1 and 1 and is defined in Equation 2.

$$
f(v)=\frac{e^{v}-e^{-v}}{e^{v}+e^{-v}}
$$

As mentioned above, the neurons are generally grouped into layers. There can be any number of layers and any number of neurons in each layer. The nomenclatures for neuron layers include the output layer, which calculates the output of the network and the hidden layer, where inputs to the neural network are presented. It is important to note that any neuron that does not provide a network output is not referred to as an output neuron, even if it is in the output layer. This will become clearer when feedback networks are described later.

These neuron layers are connected to each other through a series of synapses. The inputs to the network are also connected to the first layer through these synapses. Each synapse has a numerical weight (multiplier) associated with it. The input to the synapse is multiplied by this weight and the output is fed into a neuron.

A form of shorthand is used to describe a network, consisting of the number of neurons in the layer with layers being connected by a dash. For example, a network with three neurons in the first layer, two in the second, and one output neuron would be 
written as 3-2-1. A diagram of a typical 3-2-1 neural network with four inputs ( $x_{1}$ through $\left.x_{4}\right)$ is shown in Figure 2.4.

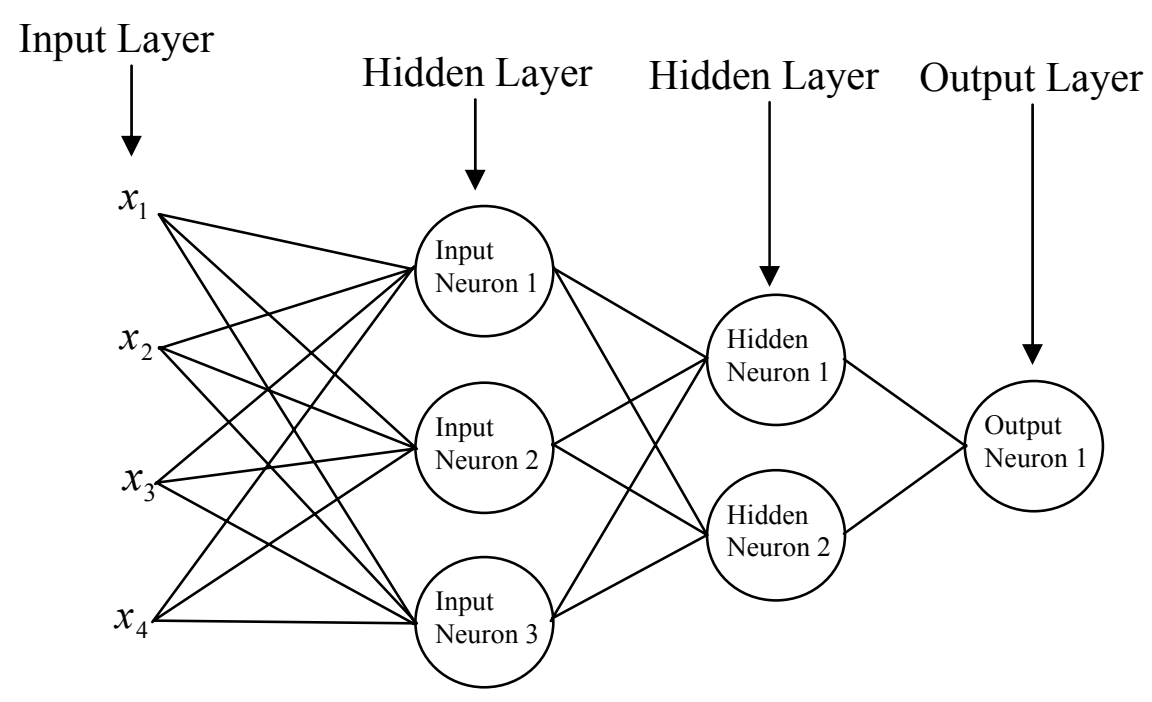

Figure 2.4: Neural Network of Architecture 3-2-1

The above neural network is a feedforward network that is fully connected; it is feedforward because the outputs of one layer feed forward to the inputs of the next layer. It is fully connected because each neuron's output in one layer is connected to every neuron's input in the proceeding layer. When this is not true, the network is considered partially connected.

A Feedback network, also called a Recurrent network, is different from a Feedforward neural network in that it has at least one feedback loop. A feedback loop occurs when a neuron's output feeds back to its own input or inputs of other neurons within the same layer or previous layers. The feedback loops involve unit-delay elements. A Recurrent network with one output neuron and one input neuron is shown in Figure 2.5 . 


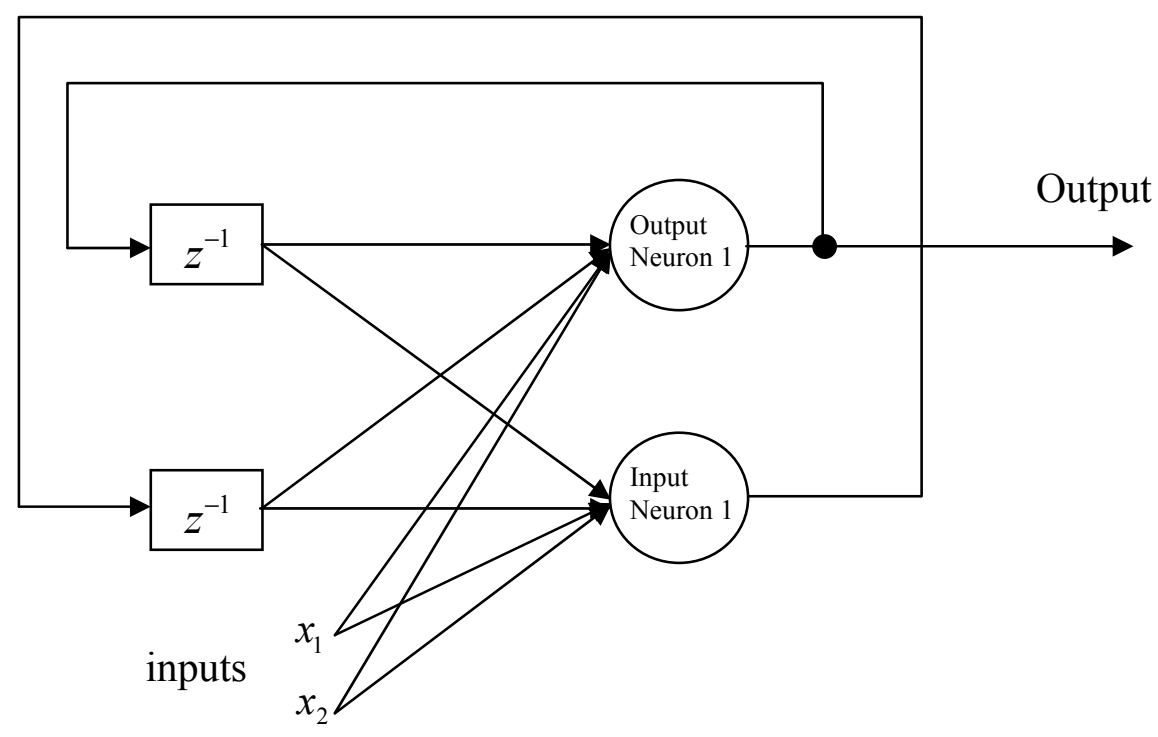

Figure 2.5: Recurrent Neural Network

If the task at hand has a temporal dimension, a network with some type of time history is required. There are two main ways of incorporating time information in a neural network (NN): Time lagged feedforward networks, and Recurrent (Feedback) Networks.

In a Focused time lagged Feedforward neural network, time delays are added at the input to the network in the form of Tapped-Delay-Lines (TDL). This creates a shortterm memory at the beginning of the network. It is important to note that focused time lagged feedforward networks (focused TLFN) are only suitable for use in stationary (i.e. time-invariant) systems [1]. Training of this type of network can be implemented using the standard back-propagation algorithm. Figure 2.6 below shows an example of a focused TLFN. 


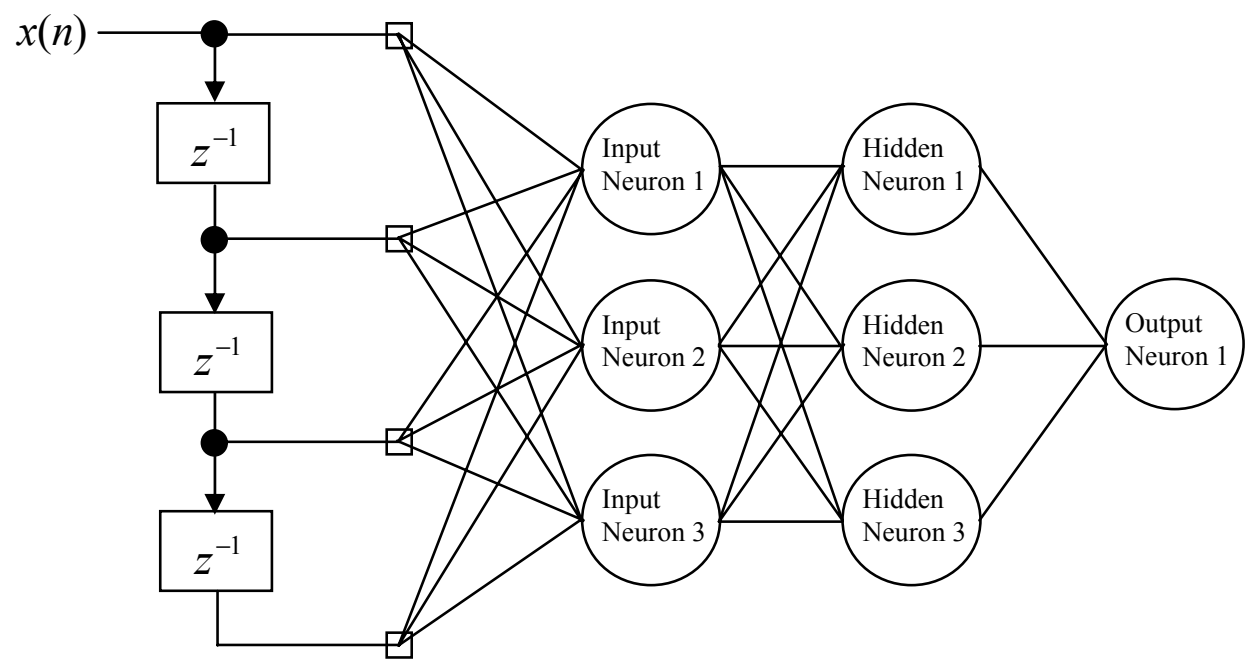

Figure 2.6: Focused Time Lagged Feedforward Neural Network

In a Distributed Time Lagged Feedforward neural network there are time delays at the beginning of the network and throughout the network. Unlike Focused TLFNs a Distributed TLFN is suitable for a dynamic system (i.e. time-variant) system [1]. This type of network relies on use of the spatio-temporal model of the neuron (multiple-input neuronal filter). This model uses finite impulse response filters (FIR) as synaptic filters. To train this type of network an elaborate learning algorithm exemplified by a temporal back-propagation algorithm is required. Due to the complexity required to train this type of network, it will not be used.

An optional but important addition to the networks described so far is the bias. The bias is an extra input applied to all or some of the neurons of a network, and is a constant. The value is usually 1 . 
The syntax for a weight is as follows: a weight $\mathrm{W}_{\mathrm{kj}}$ is associated with a synapse with input $\mathrm{x}_{\mathrm{j}}$ and this synapse goes into neuron $\mathrm{k}$. Thus, the input signal $\mathrm{x}_{\mathrm{j}}$ is multiplied by weight $\mathrm{W}_{\mathrm{kj}}$ and the resulting value is fed into the neuron.

$$
\begin{gathered}
u_{k}=\sum_{j=1}^{m} W_{k j} x_{j} \\
y_{k}=f\left(u_{k}+b_{k}\right)
\end{gathered}
$$

where $u_{k}$ in Equatin 3 is the output of the adder, $y_{k}$ in Equation 4 is the output of the neuron, $x_{1}, x_{2}, \ldots x_{m}$ are the input signals, $W_{k 1}, W_{k 2}, \ldots W_{k m}$ are the synaptic weights of neuron $\mathrm{k}, \mathrm{f}$ is the activation function, and $b_{k}$ is the bias.

\section{3 - Neural Network Training}

Many learning algorithms have been developed to train neural networks. The relationship between a neural network's inputs and its outputs can be changed to meet a desired response by adjusting the weights of the network. Currently there are three main ways a network's weights are adjusted: reinforcement learning, unsupervised learning, or supervised learning.

In reinforcement learning (reward-penalty learning) an input vector is provided to the neural network and the output of the network is calculated. If the output is considered "good" the weights receive a "reward" and their value is increased. If the output is considered "bad", the weights are "punished" and their value will be decreased. 
In unsupervised learning the neural network is provided with the input vector but not the output vector. In this type of training the network "learns" the internal features of the input vector.

Lastly, with supervised learning the neural network is provided with a training set which includes an input vector, $\mathrm{x}$, and the corresponding desired output vector, $\mathrm{y}$. The neural network "learns" to associate each input vector, $\mathrm{x}_{\mathrm{i}}$, to output vector, $\mathrm{y}_{\mathrm{i}}$, by changing the weights of the network.

In most control system applications the network is trained using supervised learning because there is some desired response of the system (target vector $\mathrm{y}_{\mathrm{i}}$ for a given input vector, $\mathrm{x}_{\mathrm{i}}$. Supervised learning only, will be considered for this reason.

To reduce error between the network's output and the desired output, the weights are adjusted. This process can continue indefinitely or until a limit is reached. This limit is usually either a user-defined minimum output error or is a maximum number of userdefined training iterations. A simple block diagram showing the process is shown in Figure 2.7.

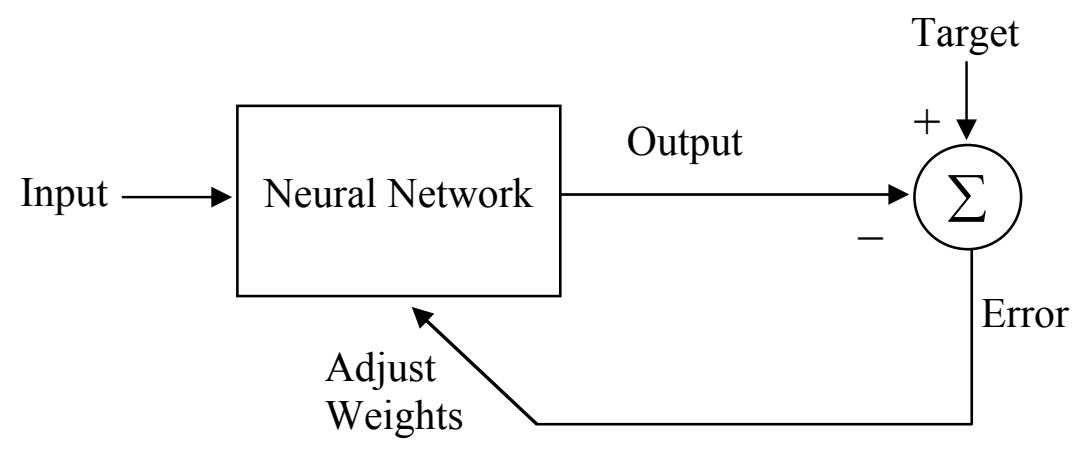

Figure 2.7: Block Diagram of Neural Network Training 
Within Supervised learning there are two main categories of training methods, in addition to some other training algorithms. The two main categories are first order gradient methods and second order gradient methods.

\subsection{1 - Backpropagation Algorithm (BP)}

The backpropagation algorithm is one of the most well known and widely used training methods. It is based on the steepest descent techniques and is extended to each layer of the network, starting at the output layer, using the chain rule. The algorithm calculates the gradient of a performance measure using the error between the desired and actual output with respect to the weights of the network. In other words, the relationship between a change in the weights and a change in error is calculated. The backpropagation process is divided into a forward pass and a backward pass. In the forward pass the networks outputs are calculated given a set of inputs. In the backward pass the weights are adjusted in order to reduce the error at the output of the network.

In order to adjust the weights of the network, a performance measure is created. This is commonly done by calculating the total error energy at the output of the network. This is accomplished by summing the error energies at each output of the neural network. The error at each output is defined as:

$$
e_{j}(n)=d_{j}(n)-y_{j}(n)
$$


Where, at output, $j, d_{j}(n)$ is the desired output, $y_{j}(n)$ is the actual output, and $n$ is the time sample. The error energy for each output of the network is defined as $\frac{1}{2} e_{j}(n)^{2}$. Thus, the total energy (performance measure) at the network output is as follows:

$$
\varepsilon(n)=\frac{1}{2} \sum_{j=1}^{J} e_{j}(n)^{2}
$$

Here, $\mathrm{J}$ is the number of network outputs, also the number of neurons in the output layer.

In order to define how well the neural network responds to all inputs or the whole data set the mean-squared-error (MSE) criterion is commonly used and is defined as:

$$
M S E=\frac{1}{N} \sum_{n=1}^{N} \varepsilon(n)
$$

In the above equation, $\mathrm{N}$ is the number of input/target pairs (input vector/target vector pairs). The MSE is an average of the total error energy at the output of the network. As the MSE is minimized the neural network will give a response closer to the desired output. To have a better idea of how this works, consider a neural network with two weights. If the total error energy at the output of the neural network is graphed in 
relation to the weights it might look like the following graph.

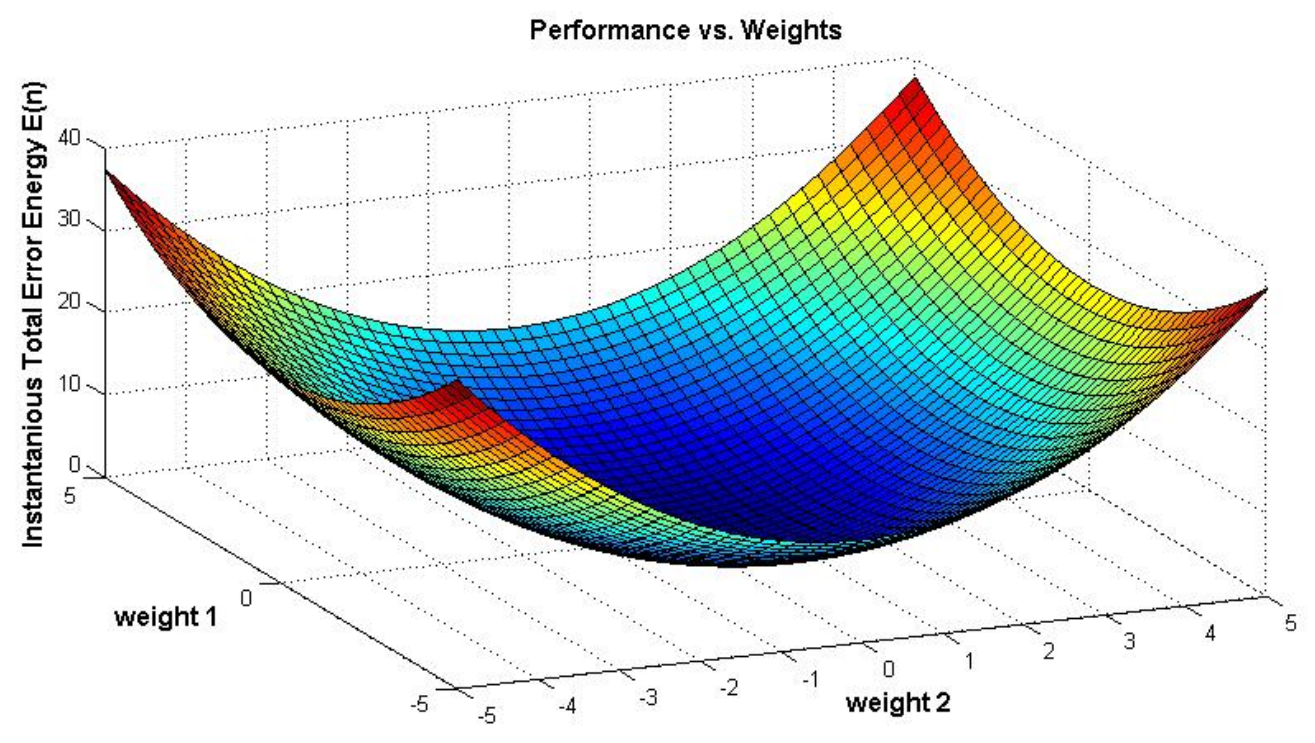

Figure 2.8: Performance vs. Weights of Neural Networks

In the above graph the lowest total error energy is obtained when both weights are zero.

If a neural network contains q weights, the goal is to find the smallest total error energy in the $(\mathrm{q}+1)$ dimensional space at a given time sample, or the smallest MSE when considering more than one time sample.

The backpropagation algorithm uses the idea of gradient descent to find the best weight values. When the neural network is first trained, the location in weight space of the MSE is random. The weights are adjusted in the direction at which the error surface decreases the most. To accomplish this, the gradient is calculated. Since the gradient is in the direction at which the error is increasing, the weights are adjusted in the opposite direction to the gradient. To help explain this, the performance measure of the 2 -weight network is plotted vs. weight 1 and weight 2 in a 2 dimensional view. Each line in the plot below represents a different value for weight 1 . 


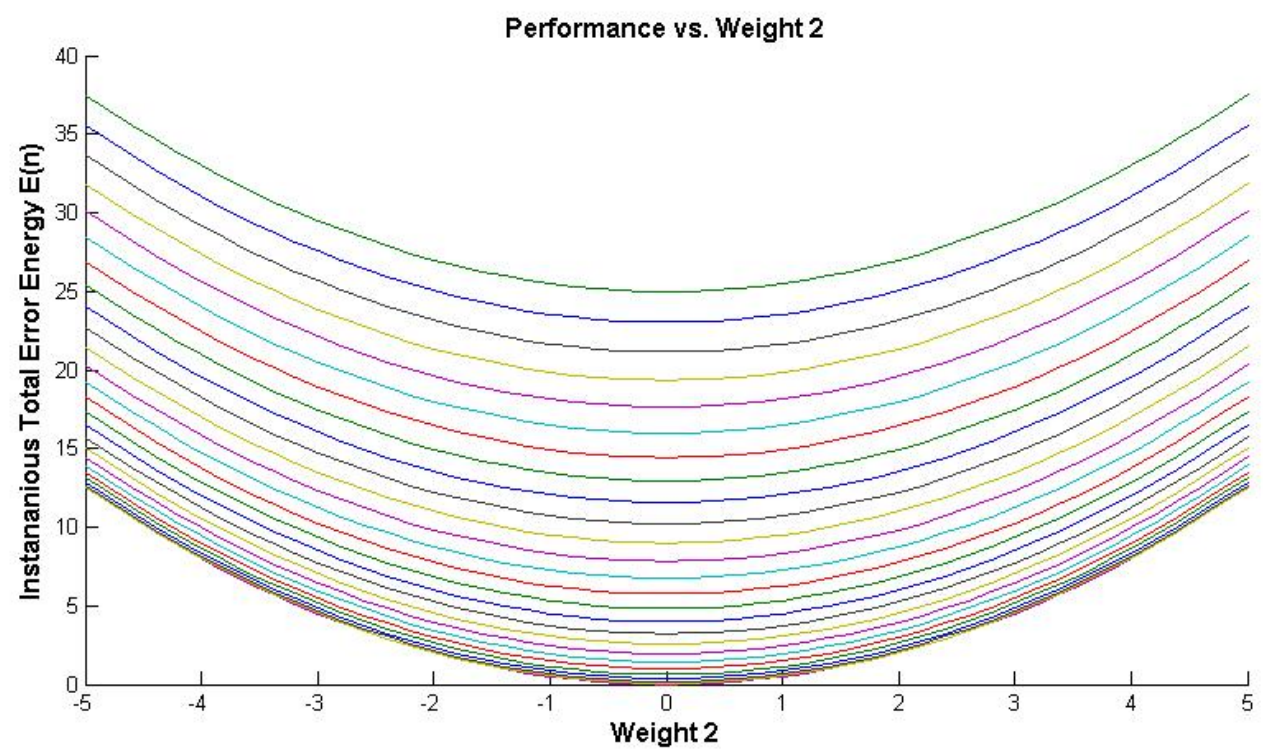

Figure 2.9: Performance vs. Weight 2

If a single point is focused on in weight space, the plot might look like that shown in Figure 2.10 below.

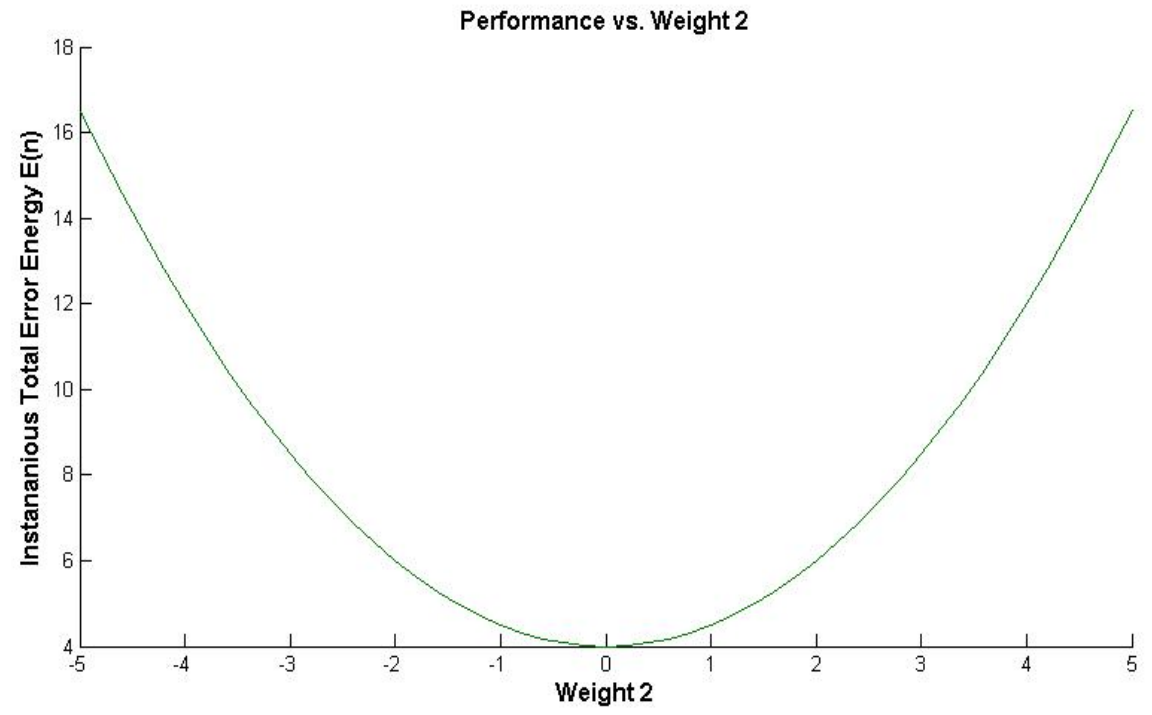

Figure 2.10: Performance vs. Weight 2 Given Weight 1 
The gradient for any one weight at a point in weight space is the derivative (slope) of the performance measure relative to that weight. Thus, as shown in the above figure, if the slope is positive the weight is decreased, and if the slope is negative the weight is increased. Note that the relationship between the weight change and the slope of performance vs. weight (gradient) is opposite in sign.

There are several steps to the backpropagation algorithm: 1) calculate the performance measure for each output, 2) calculate the derivative (slope/gradient) of the performance measure with respect to weights at the output layer of the network, 3) calculate the derivative of the performance measure in respect to all other weights in the network, 4) apply the gradients to update the weights.

\section{1) Finding the Output Performance Measure}

The output performance measure is calculated using Equation 8:

$$
\varepsilon(n)=\frac{1}{2} \sum_{j=1}^{J} e_{j}(n)^{2} \text { where, } e_{j}(n)=d_{j}(n)-y_{j}(n) \text {. }
$$

\section{2) Calculate the performance measure derivatives (gradients) for output weights}

The following figure is a signal flow graph of output neuron $\mathrm{j}$.

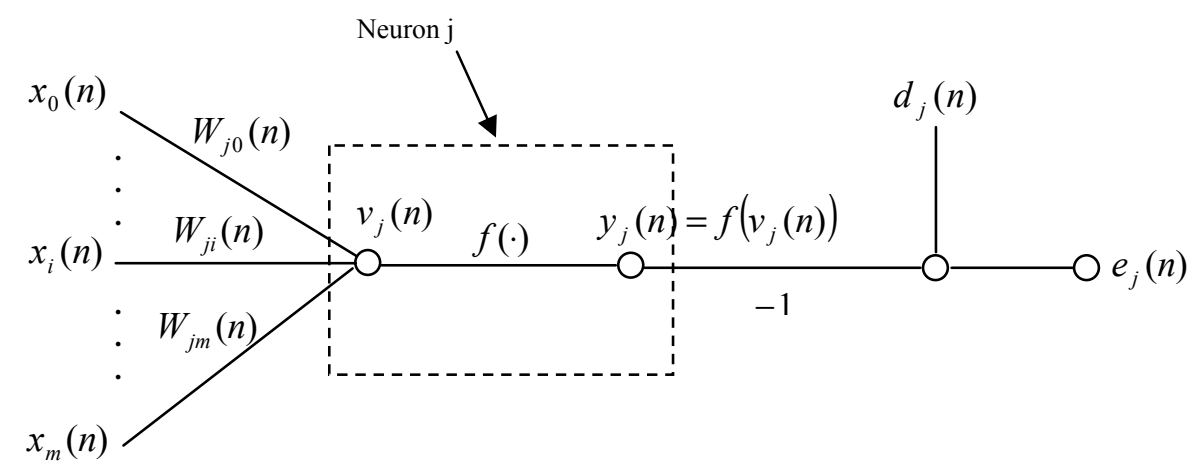

Figure 2.11: Output Neuron Signal Flow Graph 
In the above figure, $\mathrm{x}_{\mathrm{i}}(\mathrm{n})$ is the output of neuron, $\mathrm{i}$, in the previous layer, $\mathrm{W}_{\mathrm{ji}}(\mathrm{n})$ is the weight from neuron $i$ to neuron $j$. Local field $v_{j}(n)$ is the summation of inputs to neuron j. $f(\cdot)$ is the transfer function (activation function) of the neuron. $\mathrm{y}_{\mathrm{j}}(\mathrm{n})$ is the output of neuron $j \cdot d_{j}(n)$ is the desired output of the neuron. And $e_{j}(n)$ is the error at the output. Thus, $\mathrm{v}_{\mathrm{j}}(\mathrm{n})$ and $\mathrm{y}_{\mathrm{j}}(\mathrm{n})$ can be calculated as follows:

$$
\begin{aligned}
& v_{j}(n)=\sum_{i=0}^{m} w_{j i}(n) x_{i}(n) \\
& y_{j}(n)=f\left(v_{j}(n)\right)
\end{aligned}
$$

Using the chain rule and the above figure the gradient of the performance measure (total error energy) in respect to the weights between the output layer of neurons and the previous layer is calculated as:

$$
\frac{\partial \mathcal{E}(n)}{\partial w_{j i}(n)}=\frac{\partial \varepsilon(n)}{\partial e_{j}(n)} \frac{\partial e_{j}(n)}{\partial y_{j}(n)} \frac{\partial y_{j}(n)}{\partial v_{j}(n)} \frac{\partial v_{j}(n)}{\partial w_{j i}(n)}
$$

Each term on the right side of the above equation can be simplified. Taking the derivative of both sides of Equation 6, the first term can be calculated as:

$$
\frac{\partial \varepsilon(n)}{\partial e_{j}(n)}=e_{j}(n)
$$

Differentiating both sides of Equation 5 with respect to $\mathrm{y}_{\mathrm{j}}(\mathrm{n})$ results in:

$$
\frac{\partial e_{j}(n)}{\partial y_{j}(n)}=-1
$$


Differentiating both sides of Equation 12 results in:

$$
\frac{\partial y_{j}(n)}{\partial v_{j}(n)}=f_{j}^{\prime}\left(v_{j}(n)\right)
$$

Differentiating both sides of Equation 9 results in:

$$
\frac{\partial v_{j}(n)}{\partial w_{j i}(n)}=x_{i}(n)
$$

Now, rewrite Equation 11 using Equations 12 through 15 as:

$$
\frac{\partial \varepsilon(n)}{\partial w_{j i}(n)}=-e_{j}(n) f_{j}^{\prime}\left(v_{j}(n)\right) x_{i}(n) \text { (output gradient) }
$$

The derivative of transfer functions (activation functions) $f_{j}^{\prime}\left(v_{j}(n)\right)$ can be put in terms of the neurons output. The derivatives for two of the most common are shown below:

Sigmoid Function: $f_{j}\left(v_{j}(n)\right)=\frac{1}{1+e^{-a v_{j}(n)}} \quad a>0$ and $-\infty<v_{j}(n)<\infty$

$$
\begin{aligned}
& f_{j}^{\prime}\left(v_{j}(n)\right)=\frac{a e^{-a v_{j}(n)}}{\left(1+e^{-a v_{j}(n)}\right)^{2}} \\
& f_{j}^{\prime}\left(v_{j}(n)\right)=a f_{j}\left(v_{j}(n)\right) \frac{e^{-a v_{j}(n)}}{1+e^{-a v_{j}(n)}}=a f_{j}\left(v_{j}(n)\right) \frac{1+e^{-a v_{j}(n)}-1}{1+e^{-a v_{j}(n)}} \\
& f_{j}^{\prime}\left(v_{j}(n)\right)=a f_{j}\left(v_{j}(n)\right)\left[\frac{1+e^{-a v_{j}(n)}}{1+e^{-a v_{j}(n)}}-\frac{1}{1+e^{-a v_{j}(n)}}\right]=a f_{j}\left(v_{j}(n)\right)\left[1-f_{j}\left(v_{j}(n)\right)\right]
\end{aligned}
$$

the output of neuron $j=y_{j}(n)=f_{j}\left(v_{n}(n)\right)$ so:

$$
f_{j}^{\prime}\left(v_{j}(n)\right)=a y_{j}(n)\left[1-y_{j}(n)\right] \quad(\text { sigmoid function })
$$

Hyperbolic Tangent Function: $f_{j}\left(v_{j}(n)\right)=a \tanh \left(b v_{j}(n)\right) \quad(a, b)>0$ 


$$
\begin{array}{r}
f_{j}^{\prime}\left(v_{j}(n)\right)=a b \operatorname{sech}^{2}\left(b v_{j}(n)\right)=a b\left(1-\tanh ^{2}\left(b v_{j}(n)\right)\right) \\
f_{j}^{\prime}\left(v_{j}(n)\right)=\frac{b}{a}\left[a-y_{j}(n) \llbracket a+y_{j}(n)\right] \text { (hyperbolic tangent) }
\end{array}
$$

\section{3) Calculate the performance measure gradients for hidden weights}

The above equation represents the gradient for weights between the output layer and the previous layer (a hidden layer). From now on, $\mathrm{k}$ will be used to indicate a hidden neuron and $\mathrm{j}$ will be used to indicate a neuron in the next layer. The gradient for a hidden neuron is then written:

$$
\frac{\partial \varepsilon(n)}{\partial w_{k i}(n)}=-e_{k}(n) f_{k}^{\prime}\left(v_{k}(n)\right) x_{i}(n)
$$

Since there is no way to measure $e_{k}(n)$ (the error at the output at hidden neuron $\mathrm{k}$ ) it must be determined recursively in terms of the error signals of all neurons in the next layer to which that hidden neurons output is directly connected. The local gradient is defined as the derivative of the performance measure $\varepsilon(n)$ in respect to the output of the local field $v(n)$ :

$$
\delta(n)=-\frac{\partial \varepsilon(n)}{\partial v(n)}
$$

For neuron $\mathrm{k}$ the local gradient can be written as:

$$
\delta_{k}(n)=\frac{\partial \varepsilon(n)}{\partial v_{k}(n)}=e_{k}(n) f_{k}^{\prime}\left(v_{k}(n)\right)
$$

Equation 12 and 13 show that $-e_{k}(n)=\frac{\partial \varepsilon(n)}{\partial e_{k}(n)} \frac{\partial e_{k}(n)}{\partial y_{k}(n)}=\frac{\partial \varepsilon(n)}{\partial y_{k}(n)}$

Therefore, the local gradient can be written as: 


$$
\delta_{k}(n)=-\frac{\partial \varepsilon(n)}{\partial y_{k}(n)} f_{k}^{\prime}\left(v_{k}(n)\right)
$$

Note that $y_{k}(n)$ is the output of hidden neuron, k. To obtain $\frac{\partial \varepsilon(n)}{\partial y_{k}(n)}$ in Equation 19, take the partial derivative of both sides of Equation 6 (performance measure/total output error energy) with respect to the error and then divide both sides by $\partial y_{k}(n)$.

$$
\frac{\partial \varepsilon(n)}{\partial y_{k}(n)}=\sum_{j=1}^{J} e_{j} \frac{\partial e_{j}(n)}{\partial y_{k}(n)}
$$

Here, $\mathrm{k}$ is the neuron in the hidden layer and $\mathrm{j}$ is a neuron in the output layer. This equation can be rewritten as:

$$
\frac{\partial \varepsilon(n)}{\partial y_{k}(n)}=\sum_{j=1}^{J} e_{j}(n) \frac{\partial e_{j}(n)}{\partial v_{j}(n)} \frac{\partial v_{j}(n)}{\partial y_{k}(n)}
$$

In order to solve for $\frac{\partial e_{j}(n)}{\partial v_{j}(n)}$ the error (Equation 5) is rewritten:

$$
e_{j}(n)=d_{j}(n)-y_{j}(n)=d_{j}(n)-f\left(v_{j}(n)\right)
$$

The partial derivative is taken of both sides in respect to $v_{j}(n)$ :

$$
\frac{\partial e_{j}(n)}{\partial v_{j}(n)}=-f_{j}^{\prime}\left(v_{j}(n)\right)
$$

In order to obtain $\frac{\partial v_{j}(n)}{\partial y_{k}(n)}$ take the partial derivative of Equation 9

$v_{j}(n)=\sum_{k=0}^{m} w_{j k}(n) y_{k}(n)$ in terms of $y_{k}(n)$ where $\mathrm{m}$ is the total number of inputs to neuron $\mathrm{j}$ excluding the bias (because the bias does not have an output of the previous layer): 


$$
\frac{\partial v_{j}(n)}{\partial y_{k}(n)}=w_{j k}(n)
$$

Equation 23 can now be rewritten using Equations 25 and 26:

$$
\frac{\partial \varepsilon(n)}{\partial y_{k}(n)}=\sum_{j=1}^{J}-e_{j}(n) f_{j}^{\prime}\left(v_{j}(n)\right) w_{j k}(n)
$$

By comparing Equation 28 with Equation 21 (the local gradient) $\frac{\partial \mathcal{\varepsilon}(n)}{\partial y_{k}(n)}$ can be rewritten

as:

$$
\frac{\partial \varepsilon(n)}{\partial y_{k}(n)}=\sum_{j=1}^{J}-\delta_{j}(n) w_{j k}(n)
$$

Plugging Equation 28 into Equation 21, the local gradient for hidden neuron $\mathrm{k}$ can be written in terms of the local gradients of all the neurons in the next layer:

$$
\delta_{k}(n)=f_{k}^{\prime}\left(v_{k}(n)\right) \sum_{j=1}^{J} \delta_{j}(n) w_{j k}(n)
$$

The gradient for hidden neuron $\mathrm{k}$ can be written in terms of the neurons local gradient:

$$
\frac{\partial \varepsilon(n)}{\partial w_{k i}(n)}=\delta_{k}(n) y_{i}(n) \text { (hidden neuron gradient) }
$$

4) Updating the weights

Once the gradients have been calculated the weights can be updated using the using the following change in weight:

$$
\Delta w=-\eta \frac{\partial \varepsilon(n)}{\partial w_{j k}(n)}
$$

Thus, the new weight can be calculated by: 


$$
w_{\text {new }}=w_{\text {old }}-\eta \frac{\partial \varepsilon(n)}{\partial w_{j k}(n)}
$$

where $\eta$ is the learning rate and controls the algorithm's convergence speed. Usually $\eta$ is a small value between 0.001 and 0.5 , and is set by the user in a guess-and-check manner or through an adaptive learning rate algorithm.

Once the weights have been adjusted a new input/output pair (Sequential training) or a new set of input/output pairs (batch training/epoch training) are presented to the network and the procedure is repeated.

When batch training is used the average gradient at the output layer instead of the gradient for one time step is used. This means that the gradients must be stored into memory until the weights are updated. The algorithm stops when either a maximum number of iterations are reached, or the mean-squared-error reaches an acceptable value.

In summary, the weights are updated using Equation 34 below:

$$
w(n)=w(n-1)-\eta \delta(n) x_{i}(n)
$$

The local gradient $\delta(n)$ is:

$$
\begin{gathered}
\delta(n)=e_{j}(n) f_{j}^{\prime}\left(v_{j}(n)\right) \text { for neuron } j \text { in output layer } \\
\delta(n)=f_{k}^{\prime}\left(v_{k}(n)\right) \sum_{j=1}^{J} \delta_{j}(n) w_{j k}(n) \quad \text { for neuron } k \text { in hidden layer }
\end{gathered}
$$

In Equation 36, neuron $\mathrm{j}$ is in the following layer in respect to hidden neuron $\mathrm{k}$. 


\subsection{2 - Sequential vs. Batch Training}

In sequential training (training by sample) the network weights are updated after each time sample or input/output pair. In batch training (training by epoch) the weights are updated after a set of input/output pairs (one epoch) are presented. When using the backpropagation algorithm with this type of training the gradients for the output layer of the network are averaged over the training set and this average is used to update the weights. Thus, the output neuron gradients must be stored in memory until the weights are updated.

There are advantages and disadvantages to using either of the training methods. Since the weights are updated in Sequential training after every input/output pair (time sample) the weights begin to update immediately and thus the error decreases immediately. In batch training, the network is not updated until a set number of input/output pairs are presented and error reduction takes longer to occur as a result. When batch training, the weights are updated using a function proportional to the average error at the neural network output. Therefore the weight trajectory is more likely to move in a direction that will generalize the network for all input/output pairs. In other words, batch training can make the network respond better to inputs on average.

\subsection{3 - Online vs. Offline Training}

Online training occurs when the neural network is trained in real-time. That is, the number of inputs is continuous. Offline training occurs when the neural network is 
trained using a finite number of input/output pairs. Both sequential and batch training can occur during online or offline training. While offline training, the neural network can be updated after every input/output pair has been presented (sequential training) or after the whole or part of the entire data set has been presented (batch training). Batch training can be used by updating the weights after every user defined number of time samples (input/target pairs). However, with online training the neural network is usually trained sequentially.

\subsection{4 - Pre and Post Processing}

The transfer function (activation function) for each neuron is typically chosen so that it accepts an input of any range and produces an output in a strictly limited range (has a squashing effect). Although the input can be in any range, there is a saturation effect so that the neuron is only sensitive to inputs within a fairly limited range. An example of such transfer functions would be the hyperbolic tangent or the sigmoid, as shown in Figure 2.3. For this reason, pre and post processing is done in order to scale the input and output into a range such as -1 to 1 when using a hyperbolic tangent function. If the maximum and minimum values are known for the input to the network, then these values can be set to 1 and -1 respectively, and every other input is set relative to those values. When the output neuron of a network is a squashing function the output is limited in range and thus scaling is required to obtain the desired response. In order to do this, the output is usually scaled to the maximum and minimum of the target. When the range of the target is unknown (as with online training), the output neurons are usually given a linear activation function that is not limited in range. 


\subsection{5 - Weight Initialization}

Initialization of the weights of a neural network before they are optimized is imperative. When training a neural network, the goal is to find the global minimum from all possible local minimums on the error surface. Thus, it is advantageous to initialize the weights with a set of values as close to the global minimum as possible. Doing so will minimize the number of training cycles. Some methods used for initializing weights include distributing them randomly.

There is also a special method for initializing network weights. This algorithm is based upon a paper by Derrick Nguyen and Bernard Widrow presented at the proceedings of the International Joint Conference on Neural Network in 1990. The Nguyen-Widrow algorithm chooses values in order to distribute the active region of each neuron in a layer evenly across the layer's input space [5]. The advantages of this algorithm over purely random weights are fewer wasted neurons (since all are in the input space), and faster training. The algorithm works as follows: small random values are picked for the weights of the network. The weights are then modified so that the region of interest is divided into small intervals. By setting the initial weights of the first layer so that each neuron is assigned its own interval the training process can be sped up.

\section{4 - Alternative Training Method}

The backpropagation algorithm, which is based on a steepest-descent technique is easy to implement and computationally less complex than other algorithms. However, it 
has disadvantages such as: slow convergence speed, sensitivity to local minima, and instability if the learning rate is too large [2]. An alternative (the Levenberg-Marquardt algorithm) to backpropagation is used in this study.

\subsection{1 - Levenberg-Marquardt (LM)}

The Levenberg-Marquardt (LM) is a high-performance algorithm which can converge on a solution much faster than the backpropagation algorithm. It is an optimization algorithm that is based on a second derivative of the error, a characteristic of the Gauss Newton Method. The LM algorithm is regarded as an intermediate method between the steepest descent and the Gauss Newton method. The basic idea of the Gauss Newton Method is to minimize the quadratic approximation of the performance measure $\varepsilon(w)$ around the current point $w(n)$. This is done by using a Taylor series expansion of the error as a function of $\mathrm{w}$, calculating an approximated $\varepsilon(w)$ using this error, and finding the minimum by taking the derivative and setting it equal to zero. In essence, the curvature of the error surface is looked at; where it is assumed that the curvature seen is due to a parabolic bowl. The weights are then found for the bottom of this fictitious bowl and then re-evaluated again. The weights using this algorithm are updated using the following equation:

$$
\boldsymbol{w}(n+1)=\boldsymbol{w}(n)-(H(n))^{-1} \frac{\partial \varepsilon(n)}{\partial \boldsymbol{w}(n)}
$$

In the above equation, $\boldsymbol{w}(n)=\left[w_{1}(n), w_{2}(n), \ldots, w_{m}(n)\right]^{T}$ where $\boldsymbol{w}$ is a network weight vector containing all network weights, $\mathrm{n}$ is the current time sample or training pair (input/target pair) and $\mathrm{m}$ is the total number of weights in the neural network. $H(n)$ is an 
approximation to the Hessian matrix, where the Hessian matrix is the second derivative of the performance measure (cost function) with respect to the network weights. An approximation of the Hessian matrix is made because the Hessian matrix computation is very extensive, and may not be positive definite on every point of the error surface. With the Levenberg-Marquardt algorithm, the Hessian matrix is approximated using the following equation:

$$
H(n)=J^{T}(n) J(n)+\mu I
$$

In the above equation, $I$ is the identity matrix with size $\mathrm{n}$ and $\mu$ is a small constant that will be explained in further detail later. $J(n)$ is the Jacobian matrix and contains the first derivative of the output neuron errors with respect to the network weights. For sequential training this matrix is defined as:

$$
J(n)=\left[\begin{array}{llll}
\frac{\partial e}{\partial w_{1}} & \frac{\partial e}{\partial w_{2}} & \cdots & \frac{\partial e}{\partial w_{m}}
\end{array}\right]
$$

Again, $\mathrm{m}$ is the total number of weights in the network. The terms in $J(n)$ can be found by calculating the network gradients using the standard backpropagation algorithm with one modification at the output layer: the output layer local gradient is defined as $\delta_{j}^{L M}=$ $-f_{j}^{\prime}\left(v_{j}(n)\right)$, where $\mathrm{f}$ is the activation function for output neuron $\mathrm{j}$. Using this modified local gradient $\delta_{j}^{L M}$ the terms in the Jacobian matrix for the output layer are defined as $\frac{\partial e}{\partial w_{j i}}=\delta_{j}^{L M} x_{i}(n)$ where $x_{i}(n)$ is the input to weight $w_{j i}$ between output neuron $\mathrm{j}$ and neuron $\mathrm{i}$ in the previous layer. For neuron bias's the expression is defined as $\frac{\partial e}{\partial b_{j}}=\delta_{j}^{L M}$. The terms for hidden layer weights can be calculated in a similar fashion by backpropagating the local gradient through the network using Equation 36. The last term 
in Equation 37, $\left(\frac{\partial \varepsilon(n)}{\partial \boldsymbol{w}(n)}\right)$, is the gradient vector consisting of the gradients for each weight in the network. This vector is defined as $\frac{\partial \varepsilon(n)}{\partial \boldsymbol{w}(n)}=\left[\frac{\partial \varepsilon(n)}{\partial w_{1}}, \frac{\partial \varepsilon(n)}{\partial w_{1}}, \ldots \frac{\partial \varepsilon(n)}{\partial w_{m}}\right]^{T}$ where the performance/cost function $\varepsilon(n)=\frac{1}{2} \sum_{j=1}^{J} e_{j}(n)^{2}$ where $e_{j}(n)$ is the error at the output of the jth neuron in the output layer. Note that the gradient vector is equal to the Jacobian matrix multiplied by the error e(n). This relationship can be shown with the following calculation:

$$
J^{T}(n) e(n)=\left[\begin{array}{c}
\frac{\partial e(n) e(n)}{\partial w_{1}} \\
\vdots \\
\frac{\partial e(n) e(n)}{\partial w_{m}}
\end{array}\right]=\left[\begin{array}{c}
\frac{\partial\left(\frac{1}{2} e(n)^{2}\right)}{\partial w_{1}} \\
\vdots \\
\frac{\partial\left(\frac{1}{2} e(n)^{2}\right)}{\partial w_{m}}
\end{array}\right]=\left[\begin{array}{c}
\frac{\partial \varepsilon(n)}{\partial w_{1}} \\
\vdots \\
\frac{\partial \varepsilon(n)}{\partial w_{m}}
\end{array}\right]=\frac{\partial \varepsilon(n)}{\partial \boldsymbol{w}(n)}
$$

Using the equalities defined in Equations 38 and 40, Equation 35 can be re-written as:

$$
\boldsymbol{w}(n+1)=\boldsymbol{w}(n)-\left(J^{T}(n) J(n)+\mu I\right)^{-1} J^{T}(n) e(n)
$$

Introduced earlier, the value $\mu$ is a small positive value and I is the identity matrix. Note that the Gauss-Newton method does not include the $\mu I$ term. This term keeps the approximated Hessian from becoming singular. Note that when $\mu$ is zero, Equation 39 becomes the Gauss-Newton method. Also note that when $\mu$ is large, the algorithm becomes the backpropagation algorithm (a steepest gradient descent method) with learning rate $\frac{1}{\mu}$ which has a small step size. The Gauss-Newton method is faster and more accurate near an error minimum (small gradient or slope), so the goal is to shift toward the Gauss-Newton method as quickly as possible. To accomplish this the value $\mu$ can be made adaptive using the following expression: 


$$
\mu(n+1)=\left\{\begin{array}{l}
\mu(n) \beta, \varepsilon(n)>\varepsilon(n-1) \\
\mu(n) \alpha, \varepsilon(n)<\varepsilon(n-1)
\end{array}\right.
$$

Here, $\beta$ and $\alpha$ are values set by the user.

The Levenberg-Marquardt algorithm can also be used for batch training. In this form of training the weights can be updated using the following formula:

$$
\boldsymbol{w}(n+1)=\boldsymbol{w}(n)-\left(J^{T}(\boldsymbol{w}) J(\boldsymbol{w})+\mu I\right)^{-1} J^{T}(\boldsymbol{w}) \boldsymbol{e}(\boldsymbol{w})
$$

All variables and constants in the above equation are defined as they were in Equation 41 (sequential training) except for the Jacobian matrix and the error. In the above equation $\boldsymbol{e}(\boldsymbol{w})$ is a vector of the network output errors over the entire training set (instead of the error $\mathrm{e}(\mathrm{n})$ for one training pair). For batch training the Jacobian matrix is defined as:

$$
J(\boldsymbol{w})=\left[\begin{array}{cccc}
\frac{\partial e(1)}{\partial w_{1}} & \frac{\partial e(1)}{\partial w_{2}} & \cdots & \frac{\partial e(1)}{\partial w_{m}} \\
\frac{\partial e(2)}{\partial w_{1}} & \frac{\partial e(2)}{\partial w_{2}} & \cdots & \frac{\partial e(2)}{\partial w_{m}} \\
\vdots & \vdots & & \vdots \\
\frac{\partial e(N)}{\partial w_{1}} & \frac{\partial e(N)}{\partial w_{2}} & \cdots & \frac{\partial e(N)}{\partial w_{m}}
\end{array}\right]
$$

In the above equation, $\mathrm{N}=\mathrm{Q} \times \mathrm{J}$ where $\mathrm{Q}$ is the total number of training pairs in the training set and $\mathrm{J}$ is the number of network outputs [21]. It is important to note that unlike that for sequential training the performance function for the above algorithm is defined as $\varepsilon(\boldsymbol{w})=\sum_{i=1}^{N} e_{i}^{2}(\boldsymbol{w})$. It should also be noted that the gradient of this performance function is equal to $J^{T}(n) \boldsymbol{e}(n)$ (as seen in Equation 43) [21]. 


\section{Chapter 3: Camera System Inertial Data}

\section{1 - Data Acquisition Procedure}

The model was created using data gathered from a camera system owned by Axsys Technologies (a division of General Dynamics Advanced Information Systems). Details about this system are kept confidential for proprietary reasons and because the product is controlled under the International Trade and Arms Regulation. The data was gathered in the time domain as a time history dataset and consisted of input/output pairs. The input was a voltage command (in volts) to the motor/amplifier that controls the inner azimuth gimbal. The output was the azimuth inertial rate (radians/second) measured from a gyro rate sensor located on the payload (i.e. camera).

To collect the data, a program was written and two others were modified. First, a MATLAB program was written to create the input data and to store it in an Excel file. Second, the software residing inside the gimbal electronics was modified in $\mathrm{C}$ language. These electronics included a processor and a digital to analog converter for creating the system input voltage VIN. The gimbal electronics also included the motor amplifier circuit described in Chapter 1. Third, a computer program that communicates between a personal computer and the gimbal had to be modified using $\mathrm{C}++$. The computer program was modified to read the motor command (input) data via a .csv file and to send this data down to the turret. The software was also modified to read the inertial rate (output) data from the turret and save this data to a .csv file. The code in the turret was modified to receive this data and output it to the motors. 
The procedure for obtaining real system input/output data is as follows: First, the input signal VIN is generated and saved to an Excel file using the MATLAB program mentioned above. Second, this file is re-saved in a .csv format. Third, the modified Axsys Technologies computer program is used to load this .csv file and send the data to the gimbal electronics where it is stored in memory. Fourth, the computer program is used to send a "start" command to the gimbal electronics which indicates that the stored VIN signal is to be generated at the input of the system (inner gimbal azimuth axis). This command also indicates to the gimbal that the inertial rate is to be recorded and sent back to the computer. Finally, the computer program is used to save this data to a .csv file.

\section{2 - Software Tool Development}

To automate the creation of the input signals needed to create the training data, a program in MATLAB was created. The program contains a graphical user interface (GUI) that can be used to create several different types of time domain signals, save these signals to an Excel file, and plot the signal. The GUI is shown in Figure 3.1 below. 


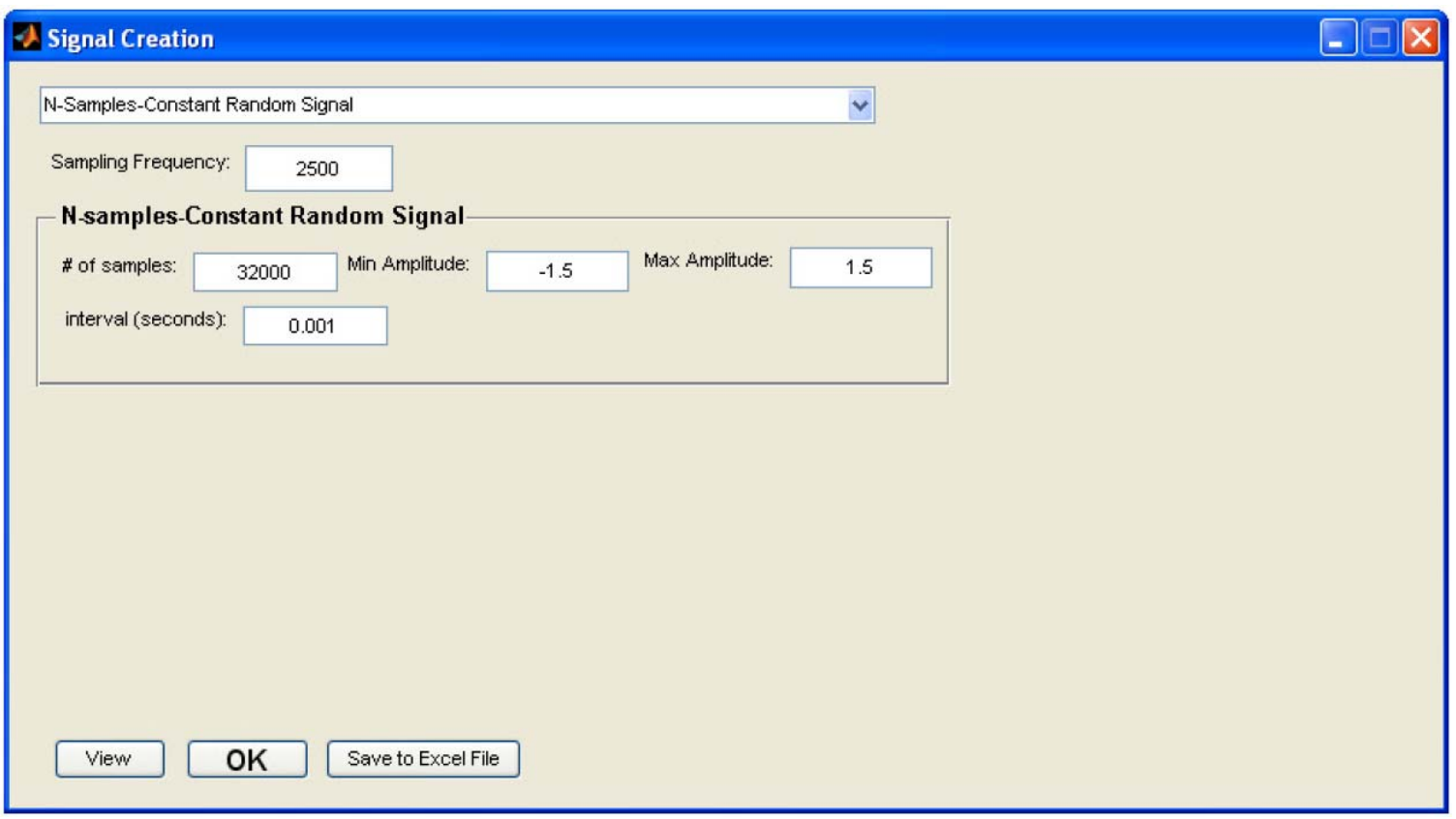

Figure 3.1: GUI for Time Domain Signal Creation Program

The GUI contains a pull down menu in the top left portion of the window that is used to select what type of signal the user wants to create. Just below the pull down menu is an input for the sampling frequency. Under the input for sampling frequency different signal parameters are visible depending upon the signal type selected. At the bottom left of the window there is a button for viewing the signal. If this button is pushed a separate figure window appears displaying the signal. The user can then zoom, scale, print, or save the figure for viewing at a later time. Also, on the bottom of the window there is a button for saving the signal to an Excel file. The signal is saved to the first column in the file. 


\section{3 - Input Signals}

There are six different types of signals that can be created using the program shown in Figure 3.1. Since the signals are generated and used in a digital system the user has the ability to set the sampling frequency $f_{s}$ for all the signals mentioned below.

The first signal consists of multiple sine waves appended where each sine wave is a different frequency. This type of signal was chosen so that the neural networks ability to model the sytem at specific system critical frequencies could be tested. The sine waves are appended to make testing more efficient. This also adds a non-linearity between sine waves.

The next signal is a multiple frequency signal. This signal was chosen so that the neural network model could be tested for a more "normal" situation where more then one frequency is present in the signal.

The third signal is a combination of the previous two. This signal consists of multiple sine waves appended with a multiple frequency wave appended at the end. This signal was chosen to test the networks ability to model changes between single and multiple frequency waves and to possibly better generalize the network model.

The fourth signal is a linear swept-frequency cosine (Chirp) signal. This signal was chosen to test the neural networks ability to model the system when frequencies are continuously changing and to test for frequencies up to the Nyquist frequency.

The fifth signal is random in amplitude and time. This signal is used to test the network models ability to identify the system when random sharp changes in amplitude 
are present as is needed in situations where the helicopter suddenly changes direction or a torque disturbance such as coulomb friction is present. It is also chosen for the purpose of training the network model so that it better generalizes the system.

The sixth and last signal is a step function. A step function is used to test the dampening effect of the model compared to the real system.

\subsection{1 - Multiple Sine Waves Appended}

The first signal consists of up to six sine waves of different frequencies adjacent to each other. The signal is defined as:

$$
x(t)=\left\{\begin{array}{crr}
\mathrm{A} \sin \left(2 \pi f_{1} t\right), & 0 \leq \mathrm{t}<t_{1} \\
\mathrm{~A} \sin \left(2 \pi f_{2} t\right), & \vdots & t_{1} \leq \mathrm{t}<2 t_{1} \\
& \\
\mathrm{~A} \sin \left(2 \pi \mathrm{f}_{\mathrm{p}} \mathrm{t}\right), & (\mathrm{p}-1) \mathrm{t}_{1} \leq \mathrm{t} \leq \mathrm{pt}_{1}
\end{array}\right.
$$

In the above equation: $f_{1}$ through $f_{p}$ are the frequencies $(\mathrm{Hz})$ for each sine wave where $p$ is the number of frequencies from one to six, $\mathrm{A}$ is the amplitude of the signal, and $t_{1}$ is the length of time at each frequency. The user can set the value for A, the number of frequencies, the value for each frequency, and the time duration $t_{1}$ for each frequency.

\subsection{2 - Multiple Frequency Wave}

The second signal consists of a multiple frequency sine wave consisting of up to six frequencies. The signal is defined as:

$$
x(t)=\sum_{n=1}^{n=N} A \sin \left(2 \pi f_{n} t\right), 1 \leq t \leq t_{1}
$$


In the above equation $t_{1}$ is the total time duration of the signal, $\mathrm{A}$ is the amplitude, $f_{n}$ is a frequency $(\mathrm{Hz})$ and $\mathrm{N}$ is the number of frequencies in the signal. The user sets the value for $t_{1}, \mathrm{~N}, \mathrm{~A}$, and $f_{n}$.

\subsection{3 - Multiple Sine Waves Appended and Multiple Frequency Wave Appended}

The third signal consists of up to six sine waves of different frequencies and a multiple frequency wave appended on the end. The multiple frequency wave can consist of up to six frequencies. This signal is defined as:

$$
x(\mathrm{t})=\left\{\begin{array}{lr}
\mathrm{A} \sin \left(2 \pi f_{1} \mathrm{t}\right), & 0 \leq \mathrm{t}<\mathrm{t}_{1} \\
\mathrm{~A} \sin \left(2 \pi f_{p} \mathrm{t}\right), & (\mathrm{p}-1) t_{1} \leq \mathrm{t}<\mathrm{p} t_{1} \\
\sum_{\mathrm{n}=1}^{\mathrm{n}=\mathrm{N}} \mathrm{B} \sin \left(2 \pi f_{n}\right), & \mathrm{p} t_{1} \leq \mathrm{t} \leq t_{2}
\end{array}\right.
$$

Here, $f_{1}$ through $f_{p}$ are frequencies $(H z)$ for each sine wave where $p$ is the number of frequencies from one to six, $\mathrm{A}$ is the amplitude of the sine waves, $t_{1}$ is the length of time at each frequency, $f_{n}$ is a frequency used in the multiple frequency wave, $\mathrm{N}$ is the number of frequencies in the multiple frequency wave (one to six), B is the amplitude of the multiple frequency wave, and $f_{2}$ is the duration of the multiple frequency wave. The user sets the value of $\mathrm{p}, \mathrm{N}, \mathrm{A}, \mathrm{B}, \mathrm{f}_{1}$ through $\mathrm{f}_{\mathrm{p}}$ for the single frequency sines waves, $f_{1}$ through $f_{N}$ for the multiple frequency wave, the number of time samples $m$ for each single frequency $\left(t_{1}=\frac{m}{f_{s}}\right)$, and the time duration for the multiple frequency wave $\left(t_{2}-p t_{1}\right)$. 


\subsection{4 - Chirp Signal}

The fourth signal is a linear swept-frequency cosine (Chirp) signal. The amplitude and time duration of the signal is adjustable along with the frequency range. The equation for this signal is shown below.

$$
x(t)=A \cos \left(\pi\left(f_{0}+\left(\frac{f_{1}-f_{0}}{\frac{t_{1}}{2}}\right) t\right) t\right), \quad 0 \leq t \leq t_{1}
$$

In the above equation, $\mathrm{A}$ is the amplitude, $f_{0}=0 \mathrm{~Hz}$ (frequency at time 0 ), $f_{1}=\frac{f_{S}}{2}$

(frequency at time $\frac{t_{1}}{2}$ ) and $t_{1}$ is the total time duration of the signal. Note that $f_{S}$ is the sampling frequency set by the user and that the total frequency range is between $f_{0}$ and $f_{1}$. Also note that frequency of the cosine signal is $\frac{1}{2}\left(f_{0}+\left(\frac{f_{1}-f_{0}}{\frac{t_{1}}{2}}\right) t\right)$ at time t. The user sets the values for $\mathrm{A}, f_{s}$, and $t_{1}$.

\subsection{5 - Appended Multiple Random Amplitude \& Random Interval (ARARI)}

The fifth signal is random in amplitude and changes amplitude at random times. The minimum and maximum time duration at a given amplitude and the amplitude limits are defined by the user. In addition, the amplitude limits can be set differently for up to four different settable time segments. The signal is defined as: 


$$
\begin{aligned}
& x(t)=\left\{\begin{array}{lr}
a_{1}+\left(b_{1}-a_{1}\right) \cdot \operatorname{rand}(1), & 0 \leq t<t_{1} \\
a_{m}+\left(b_{m}-a_{m}\right) \cdot \operatorname{rand}(1), & t_{m-1} \leq t<t_{m} \\
a_{p}+\left(b_{p}-a_{p}\right) \cdot \operatorname{rand}(1), & t_{p-1} \leq t \leq t_{p}
\end{array}\right. \\
& \text { where } \\
& t_{m}=t_{m-1}+i_{1}+\left(i_{2}-i_{1}\right) \cdot \operatorname{rand}(1) \\
& \text { and } \\
& a_{m}=\left\{\begin{array}{cc}
\alpha_{1}, & 0 \leq t<t_{1} \\
\alpha_{n}, \quad t_{s_{n-1}} \leq t \leq t_{s_{n}}
\end{array}\right. \\
& \text { and } \\
& b_{m}=\left\{\begin{array}{rr}
\beta_{1}, \quad & \leq \leq t<t_{1} \\
\beta_{n}, \quad t_{s_{n-1}} \leq t \leq t_{s_{n}}
\end{array}\right.
\end{aligned}
$$

Here, $\operatorname{rand}()$ is a Matlab function and rand(1) is a uniformly distributed pseudorandom value from 0 to $1, a_{m}$ is the minimum random amplitude and $b_{m}$ is the maximum random amplitude during the time interval $t_{m}-t_{m-1}$. Time $t_{m}=t_{m-1}+i_{1}+\left(i_{2}-i_{1}\right)$. rand(1) where value $i_{1}$ is the minimum random time duration at a given amplitude and $i_{2}$ is the maximum random time duration at a given amplitude. The time index $\mathrm{m}$ ranges between 1 and the maximum number of time intervals $p$. Note that the value $p$ is not determined until the signal is generated as it depends on the duration of all time intervals $\left(t_{m}-t_{m-1}\right)$, which are set randomly during signal generation. The amplitude limits $a_{m}$ and $b_{m}$ are determined by the user-defined constants $\alpha_{1}$ to $\alpha_{n}$ and $\beta_{1}$ to $\beta_{n}$ respectively, where $\mathrm{n}$ ranges between 1 and 4 . Note that the total signal duration $t_{p}=t_{s_{n}}$. The user 
sets the values for $i_{1}, i_{2}, \mathrm{n}$ (1 to 4$), \alpha_{1}$ to $\alpha_{n}, \beta_{1}$ to $\beta_{n}$, and the time segment duration for the different amplitude limits by setting $t_{s_{1}}$ through $t_{s_{n}}$.

\subsection{6 - Step Function}

The last signal is a step function. The equation for the function is shown below.

$$
x(t)= \begin{cases}0, & 0 \leq t<t_{1} \\ A, & t_{1} \leq t \leq t_{2}\end{cases}
$$

In the above equation: $\mathrm{A}$ is the amplitude of the step, $t_{1}$ is the time before the step and $t_{2}$ is the total signal time. The user sets the values for $\mathrm{A}, t_{1}$ and $t_{2}$.

\section{4 - Experimental Data Description}

Several types of input/output data sets are gathered from the system. All input signals are created using a $2500 \mathrm{~Hz}$ sampling rate because this is the actual closed loop rate during normal system operation.

The type of data used to simulate the model include a $5 \mathrm{~Hz}$ sine wave (Section 3.3.1), a $35 \mathrm{~Hz}$ sine wave (Section 3.3.1), a multiple frequency wave (Section 3.3.2), appended sine waves with appended multiple frequency wave (Section 3.3.3), a chirp signal (Section 3.3.4), random signal (Section 3.3.5), and a step function (Section 3.3.6). Multiple types of signals were chosen for the purpose of testing and comparing how well the network can generalize to different types of signals. 


\section{5 - Experimental Data Measurement Results}

For this study, a MATLAB program was created to automate training of the neural network and to plot the results as well as the experimentally measured data. The program is introduced in more detail in Chapter 4. The results of the experimentally measured data are shown in the following figures.

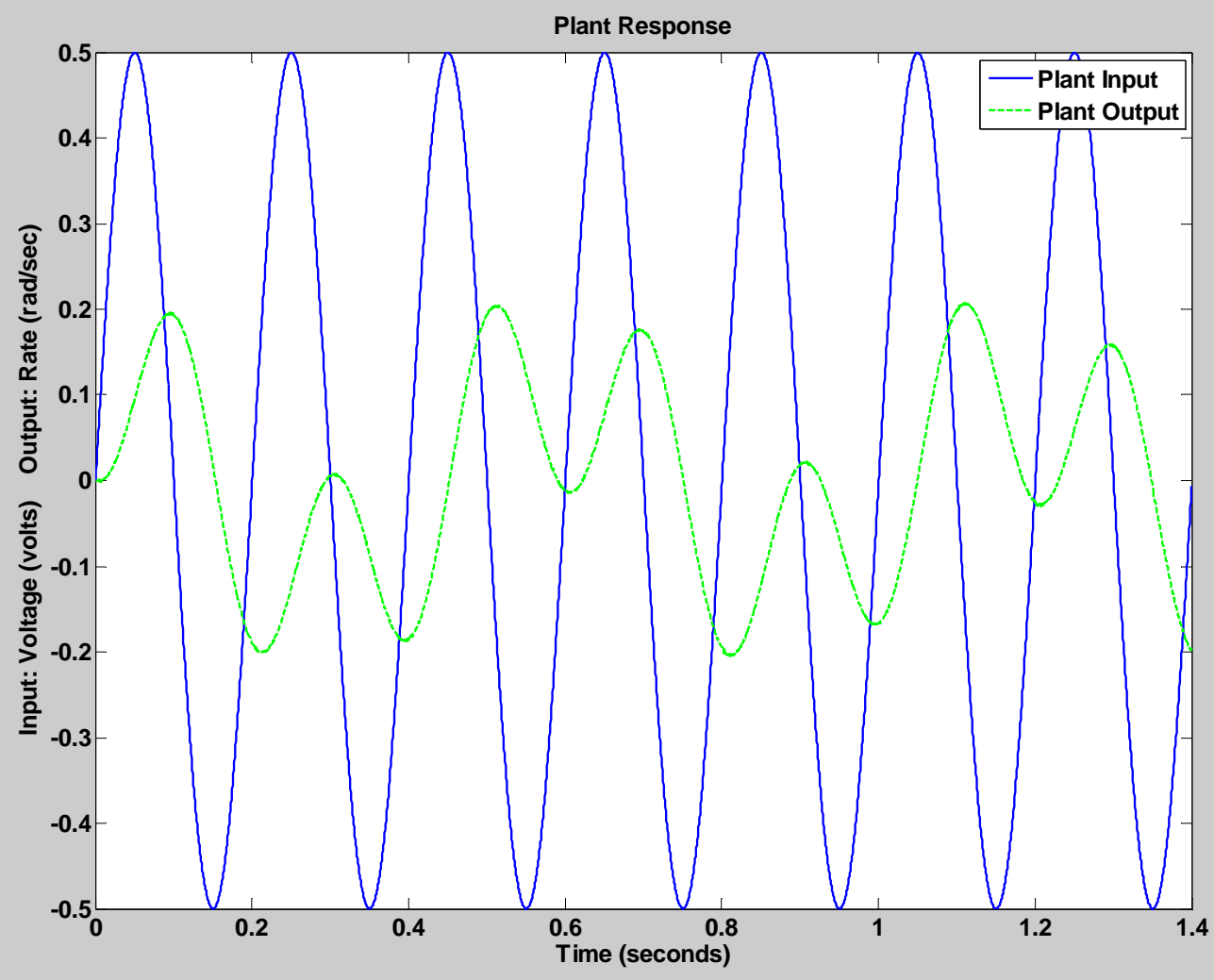

Figure 3.2: Plant Response: 5 Hz Sine Wave (Section 3.3.1)

Figure 3.2 shows the inner azimuth gimbal inertial response to a $5 \mathrm{~Hz}$ sine wave.

The signal was described in Section 3.3.1. The equation is defined as: $x(t)=$ $0.5 \sin (2 \pi \cdot 5 \cdot t), \quad 0 \leq t<1.4$. The sampling frequency $f_{s}=2500 \mathrm{~Hz}$, and total number of samples $=3500$. 


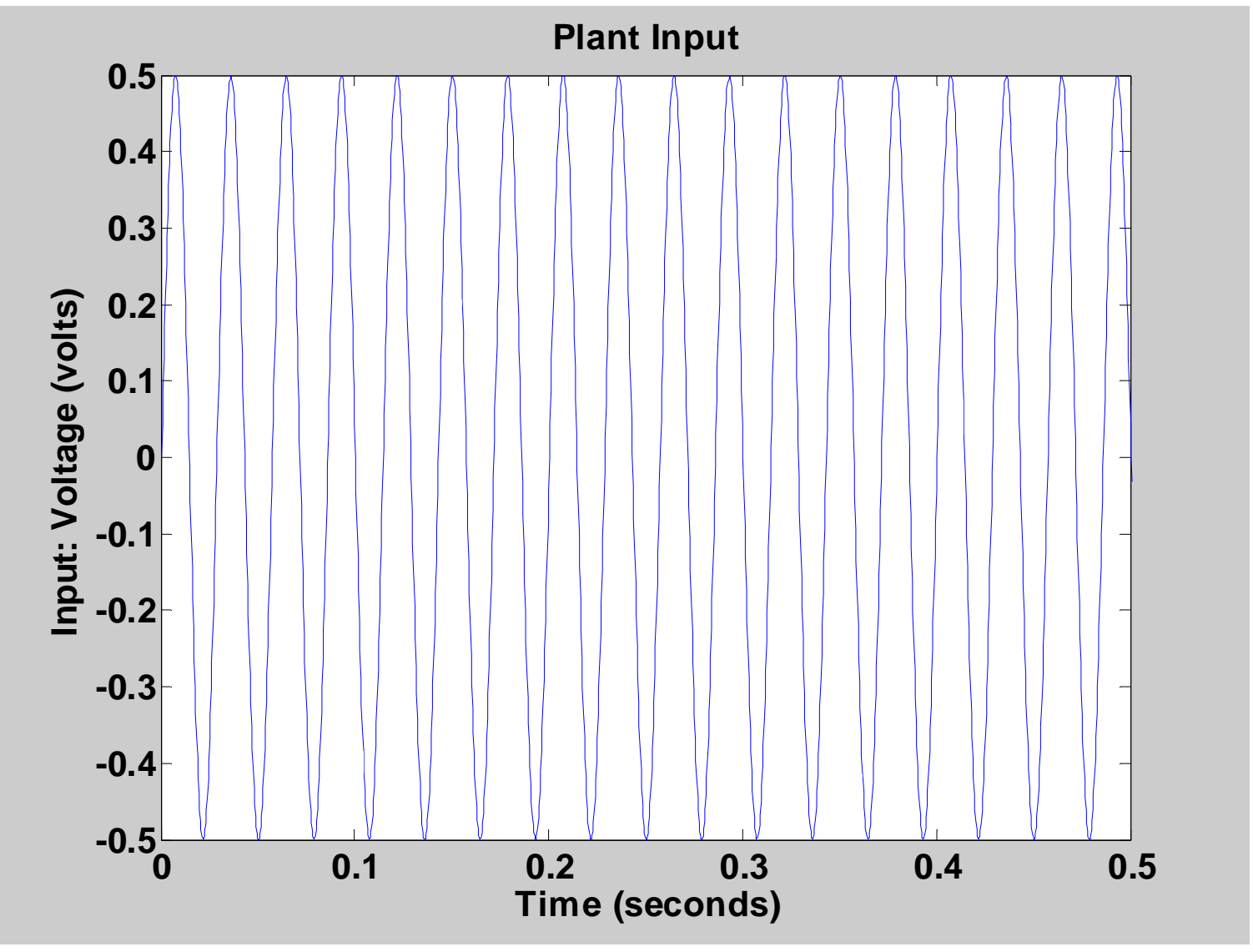

Figure 3.3: Plant Input: $35 \mathrm{~Hz}$ Sine Wave (first .5 seconds out of 12) 


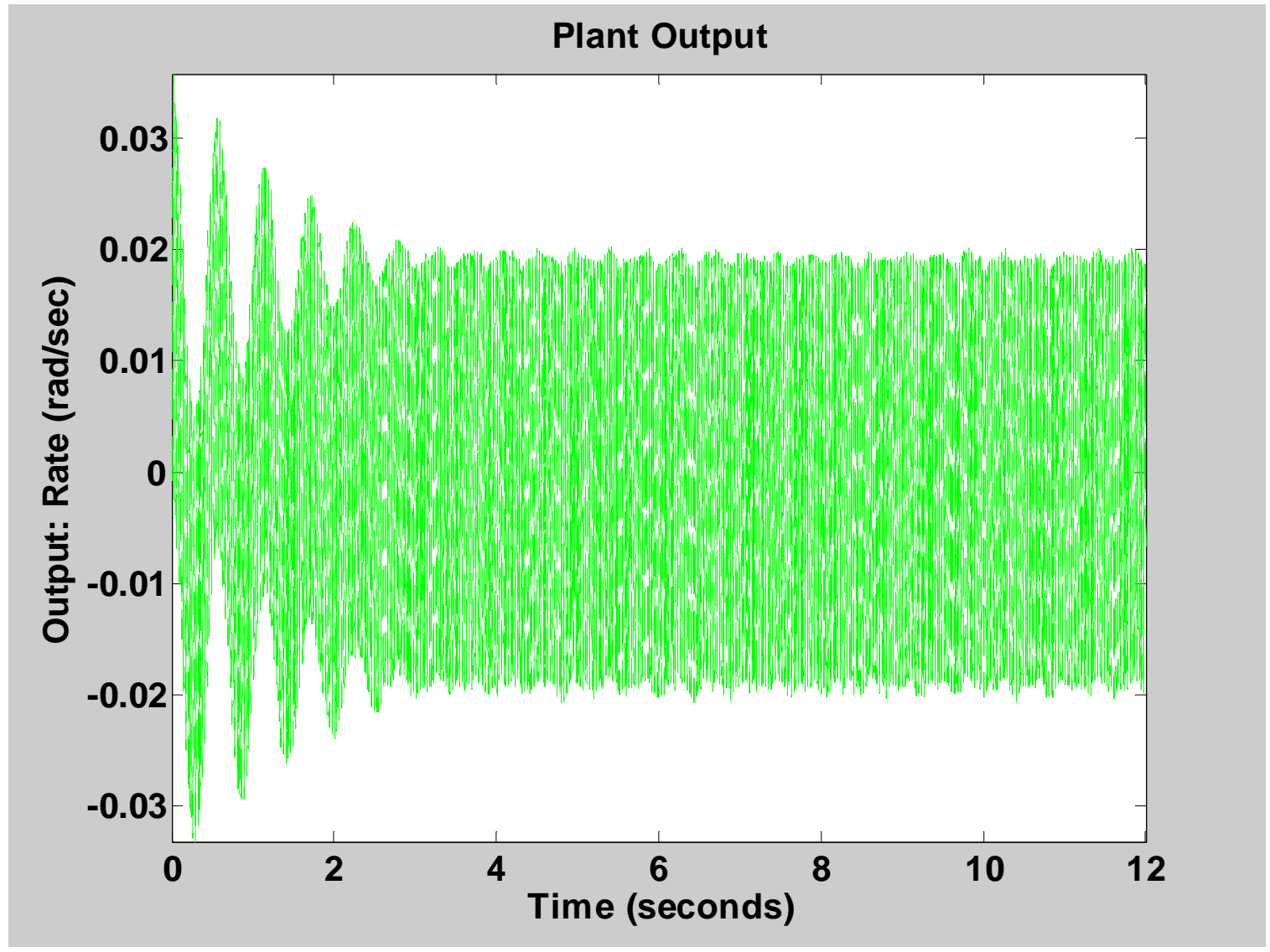

Figure 3.4: Plant Output: 35 Hz Sine Wave Input 


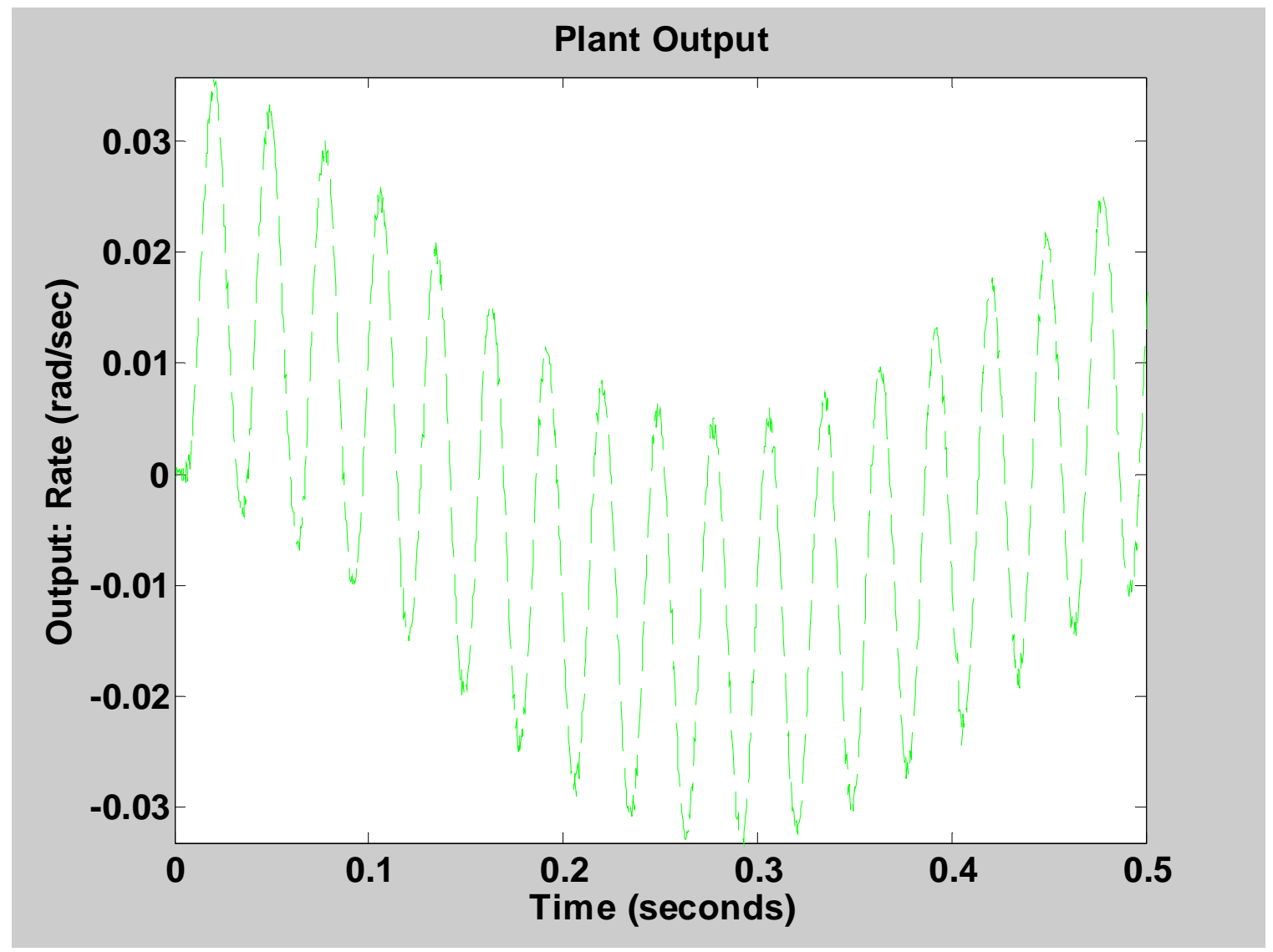

Figure 3.5: Plant Output: $35 \mathrm{~Hz}$ Sine Wave Input (first 0.5 seconds out of 12)

Figure 3.3Error! Reference source not found. shows the first 0.5 seconds (12 econd signal) of the $35 \mathrm{~Hz}$ sine wave input to the inner azimuth gimbal. Figure 3.3 through Figure 3.5 shows the gimbal resonse for the full 12 seconds and Figure 3.5 shows the reponse for the first 0.5 seconds. The input signal is also described in Section 3.3.1. The equation for the $35 \mathrm{~Hz}$ sine wave is defined as: $x(t)=0.5 \sin (2 \pi \cdot 35 \cdot t), \quad 0 \leq t<12$. The sampling frequency $f_{s}=2500 \mathrm{~Hz}$, and total number of samples $=3000$. 


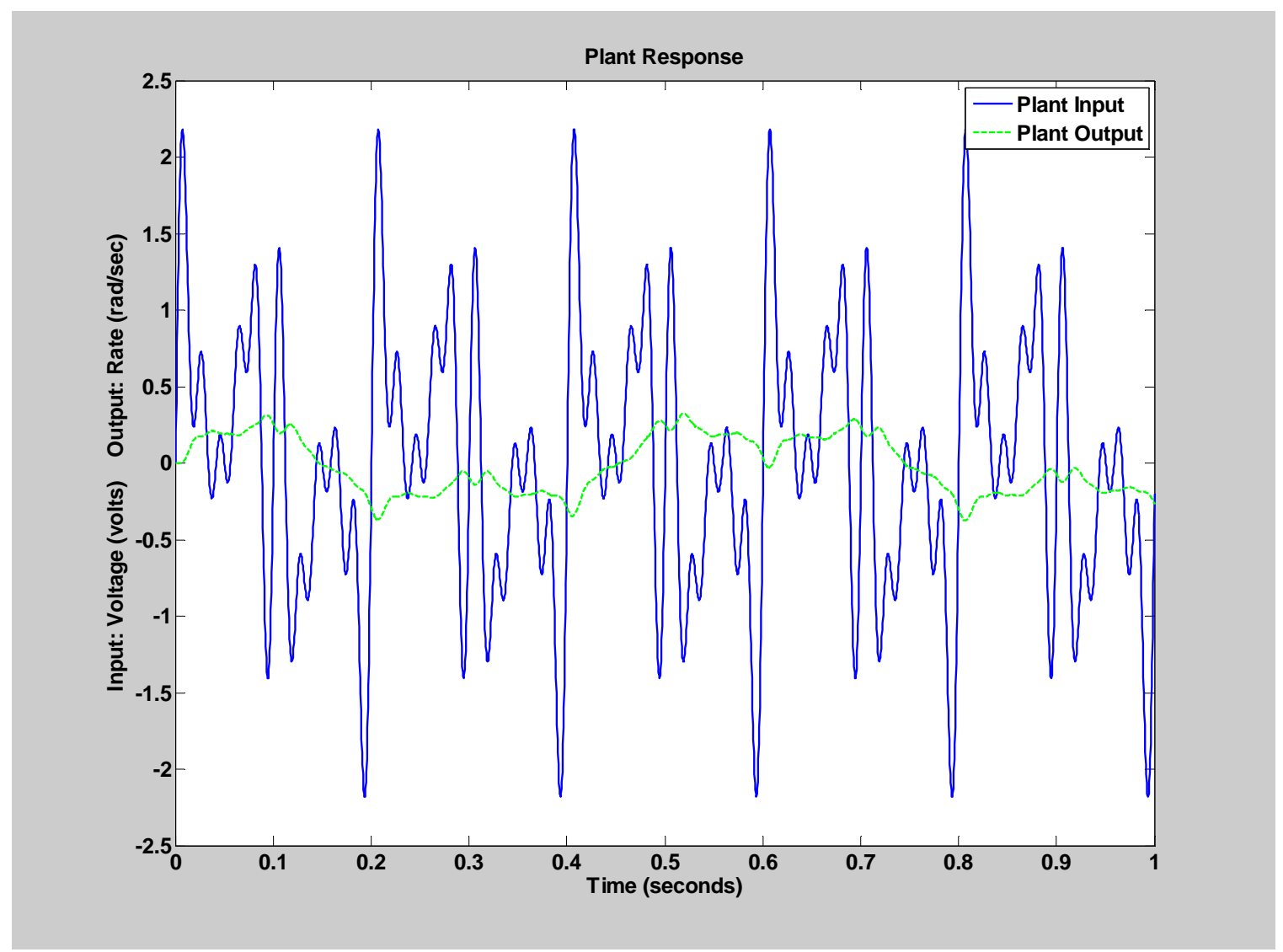

Figure 3.6: Plant Response: Multiple Frequency Wave (Section 3.3.2)

Figure 3.6 shows the inner azimuth gimbal inertial rate response to the multiple frequency input signal described in Section 3.3.2. The signal is defined as:

$x(t)=\sum_{n=1}^{n=6} 0.5 \sin \left(2 \pi f_{n} t\right), 1 \leq t \leq 1$, where $f_{n}: f_{1}=5 \mathrm{~Hz}, f_{2}=15 \mathrm{~Hz}, f_{3}=$ $20 \mathrm{~Hz}, f_{4}=30 \mathrm{~Hz}, f_{5}=40 \mathrm{~Hz}, f_{6}=50 \mathrm{~Hz}$. The total number of samples equals 2500 and the sampling rate $f_{s}=2500 \mathrm{~Hz}$. 


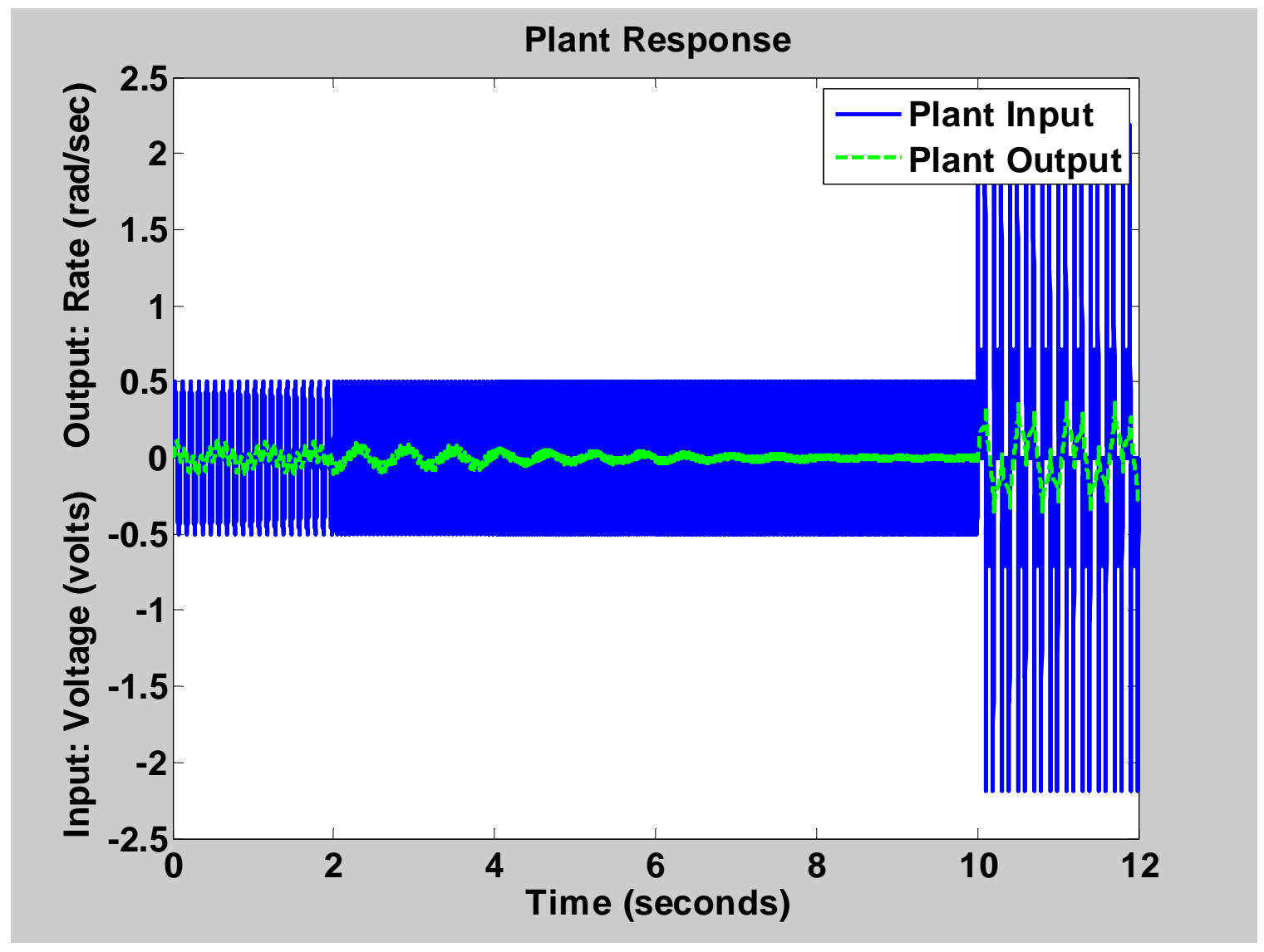

Figure 3.7: Plant Response: Single Freq with Multiple Appended (Section 3.3.3) 


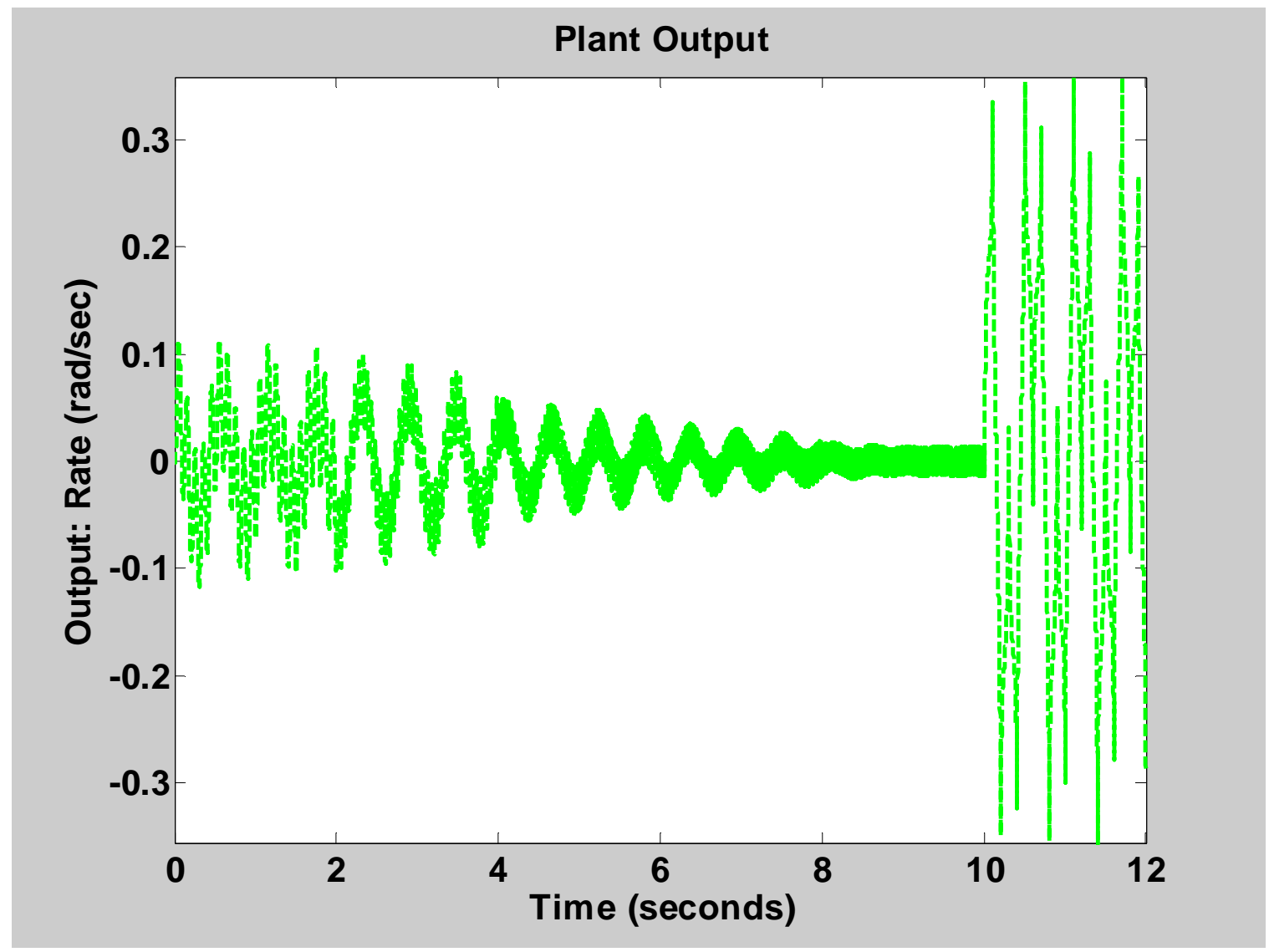

Figure 3.8: Plant Output: Single Freq with Multiple Appended Input (Section 3.3.3)

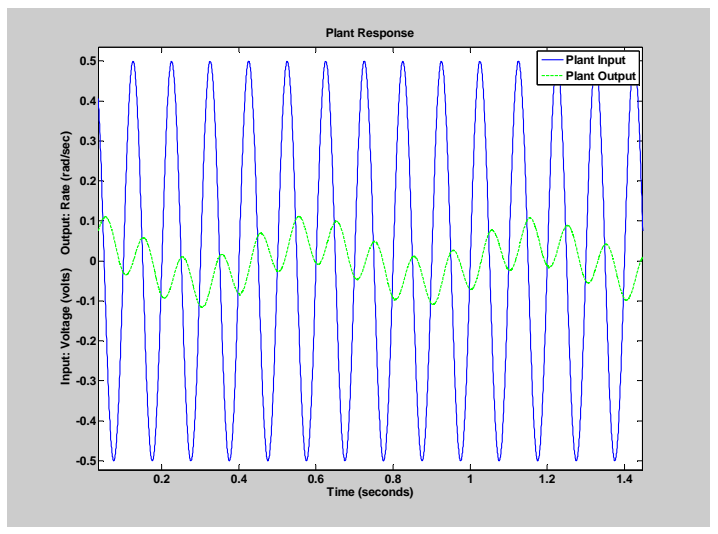

Figure 3.9: Single Freq with Multiple Appended Zoomed (10 Hz)

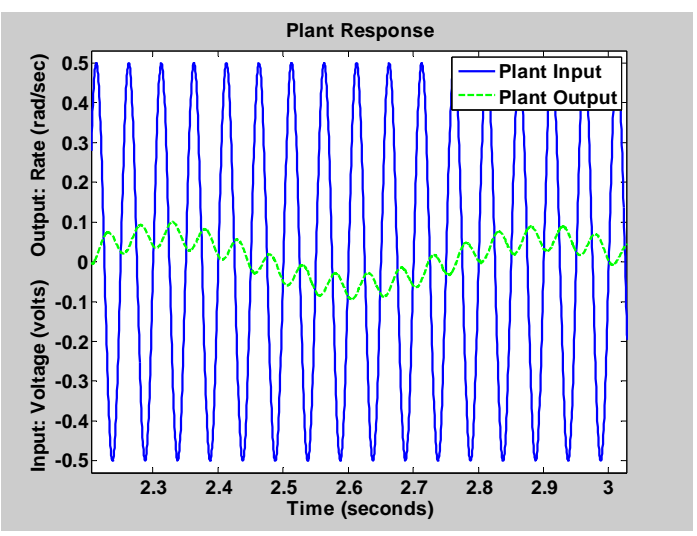

Figure 3.10: Single Freq with Multiple Appended Zoomed (20 Hz) 


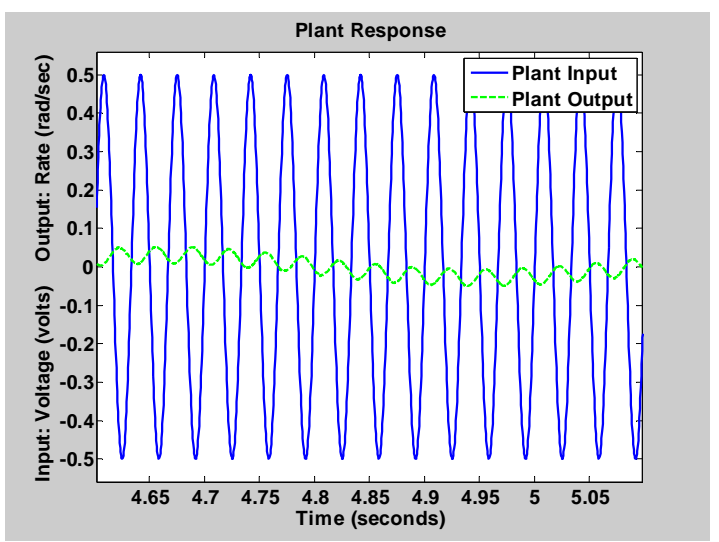

Figure 3.11: Single Freq with Multiple Appended Zoomed (30 Hz)

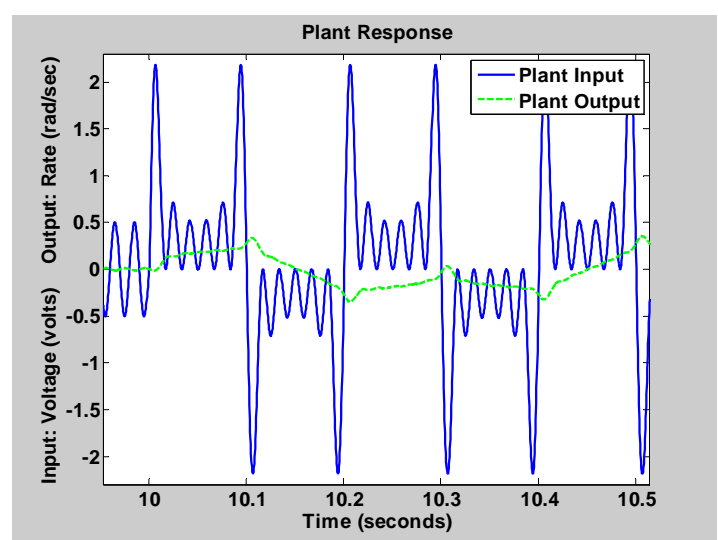

Figure 3.12: Single Freq with Multiple Appended Zoomed (50 Hz \& Multiple Freq)

Figure 3.7 shows the inner azimuth gimbal inertial response to a signal consisting of 5 appended sine waves of different frequencies and a multiple frequency wave appended at the end. Figure 3.8 shows the plant output for this input signal and more detailed views can be seen in Figure 3.9 through Figure 3.12. The signal was described in Section 3.3.3. The equation is defined as:

$$
x(t)=\left\{\begin{array}{lc}
0.5 \sin (2 \pi \cdot 10 t), & 0 \leq t<2 \\
0.5 \sin (2 \pi \cdot 20 t), & 2 \leq t<4 \\
0.5 \sin (2 \pi \cdot 30 t), & 4 \leq t<6 \\
0.5 \sin (2 \pi \cdot 40 t), & 6 \leq t<8 \\
0.5 \sin (2 \pi \cdot 50 t), & 8 \leq t<10 \\
\sum_{n=1}^{n=6} .5 \sin \left(2 \pi f_{n}\right), & 10 \leq t \leq 12
\end{array}\right.
$$

with sampling frequency $f_{s}=2500 \mathrm{~Hz}$. The total number of samples in this 12 second signal is 30,000 . The frequencies included in the multiple frequency wave were set as follows: $f_{n}: f_{1}=5 \mathrm{~Hz}, f_{2}=15 \mathrm{~Hz}, f_{3}=25 \mathrm{~Hz}, f_{4}=35 \mathrm{~Hz}, f_{5}=45 \mathrm{~Hz}, f_{6}=55 \mathrm{~Hz}$. 


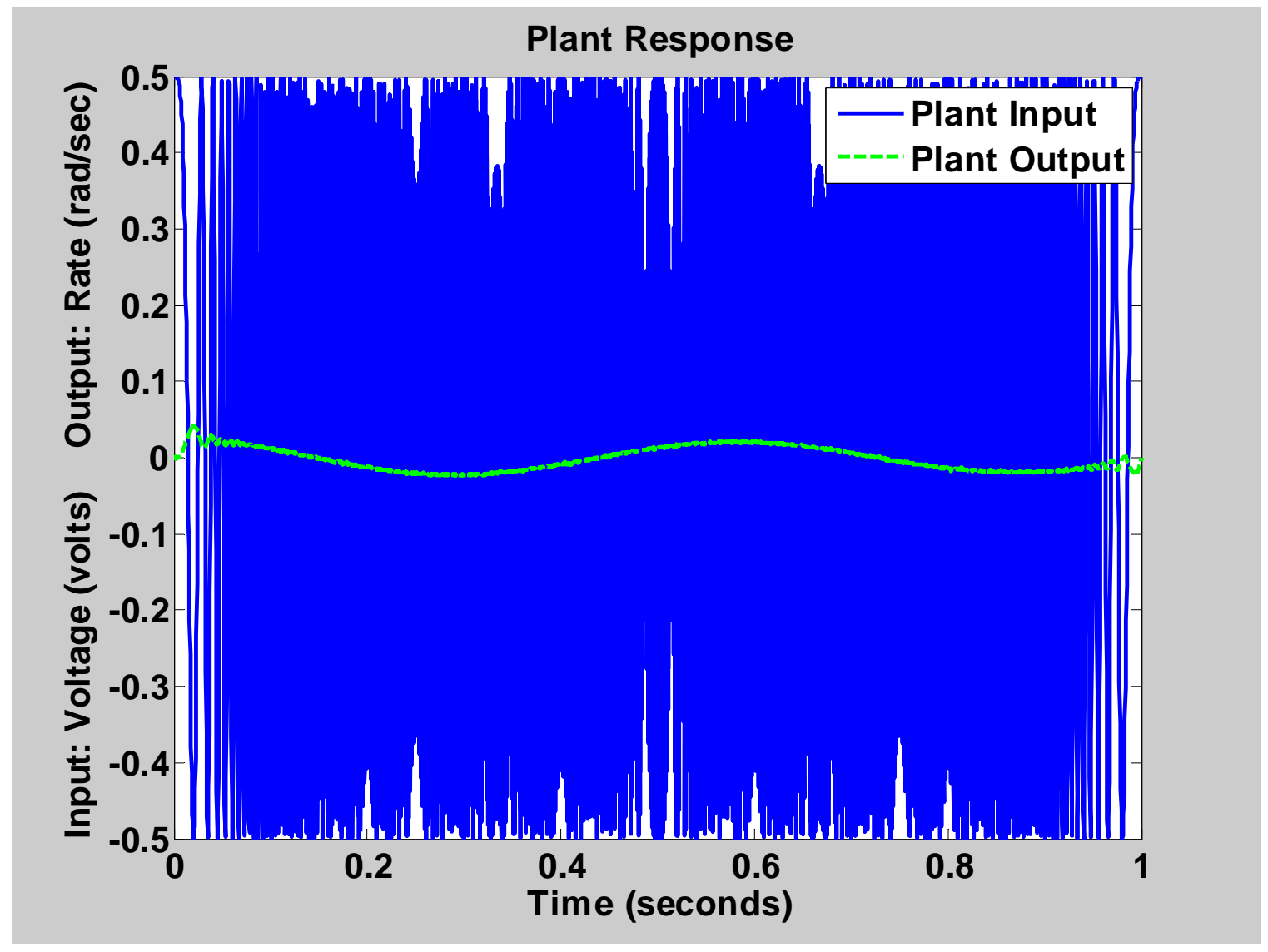

Figure 3.13: Plant Response: Chirp Signal (Section 3.3.4)

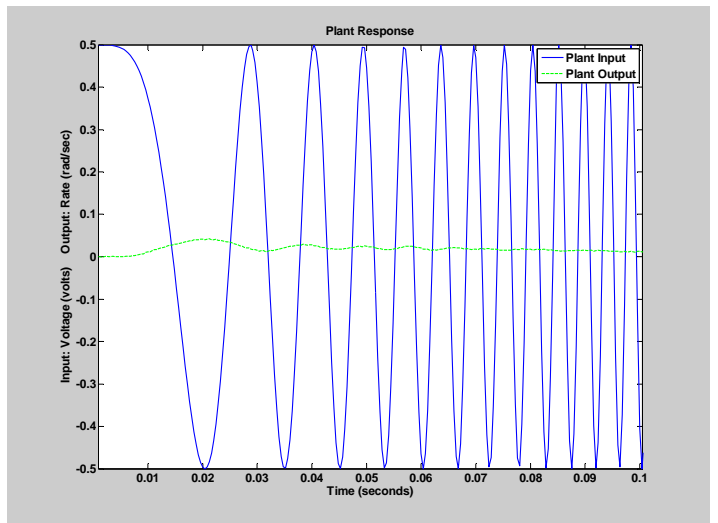

Figure 3.14: Chirp Zoomed: Low Freq

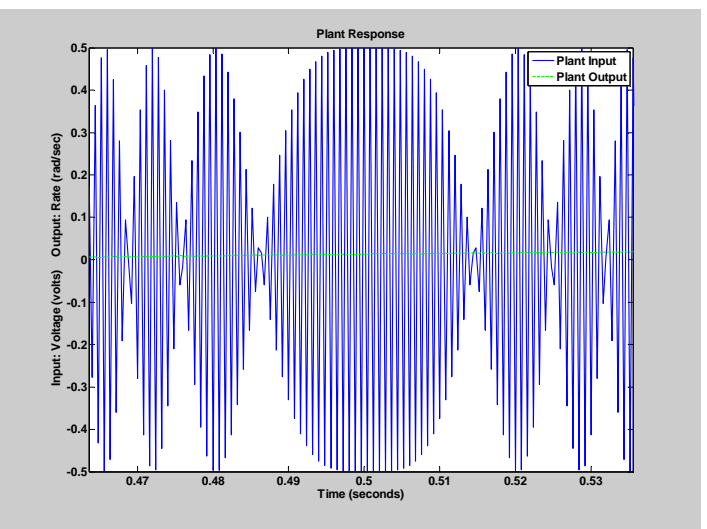

Figure 3.15: Chirp Zoomed: High Frequency 


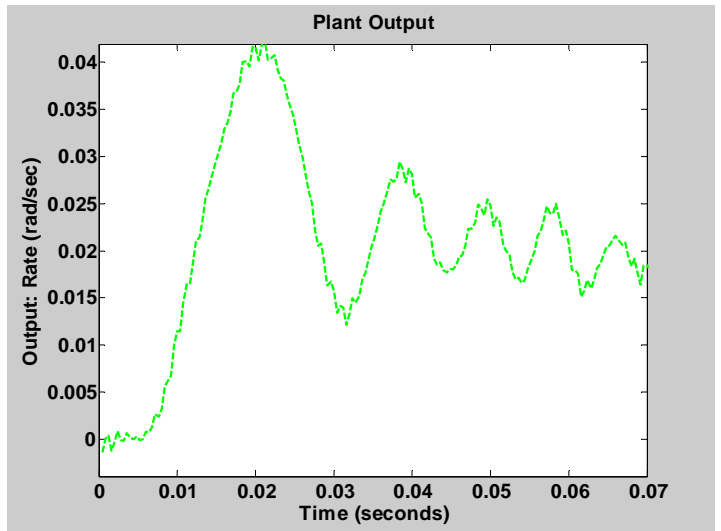

Figure 3.16: Chirp Response: Output at Low Freq

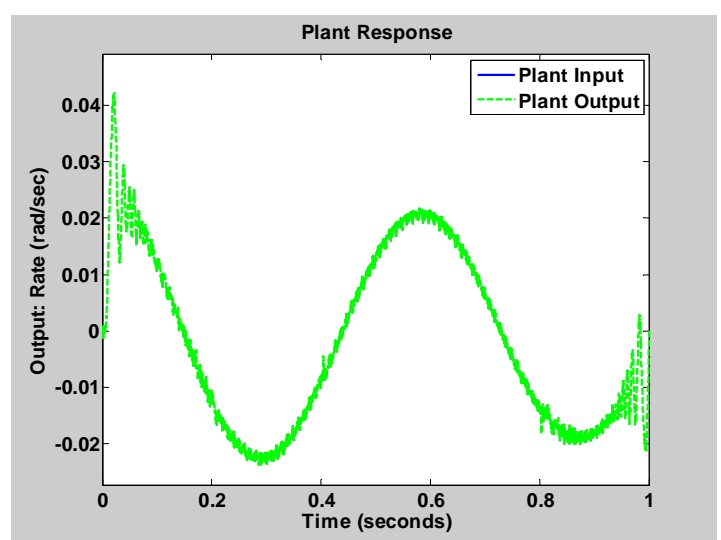

Figure 3.17: Chirp Response: Output Zoomed

Figure 3.13 shows the inertial rate output (radians/second) of the inner azimuth gimbal due to a voltage command in the form of a chirp signal. More detailed views can be seen in Figure 3.14 through Figure 3.17. The expression for the input voltage (chirp signal) is defined as follows: $x(t)=.5 \cos \left(\pi\left(0+\left(\frac{1250-0}{\frac{1}{2}}\right) t\right) t\right), \quad 0 \leq t \leq 1$. Note again that the sampling frequency $f_{s}=2500 \mathrm{~Hz}$. 


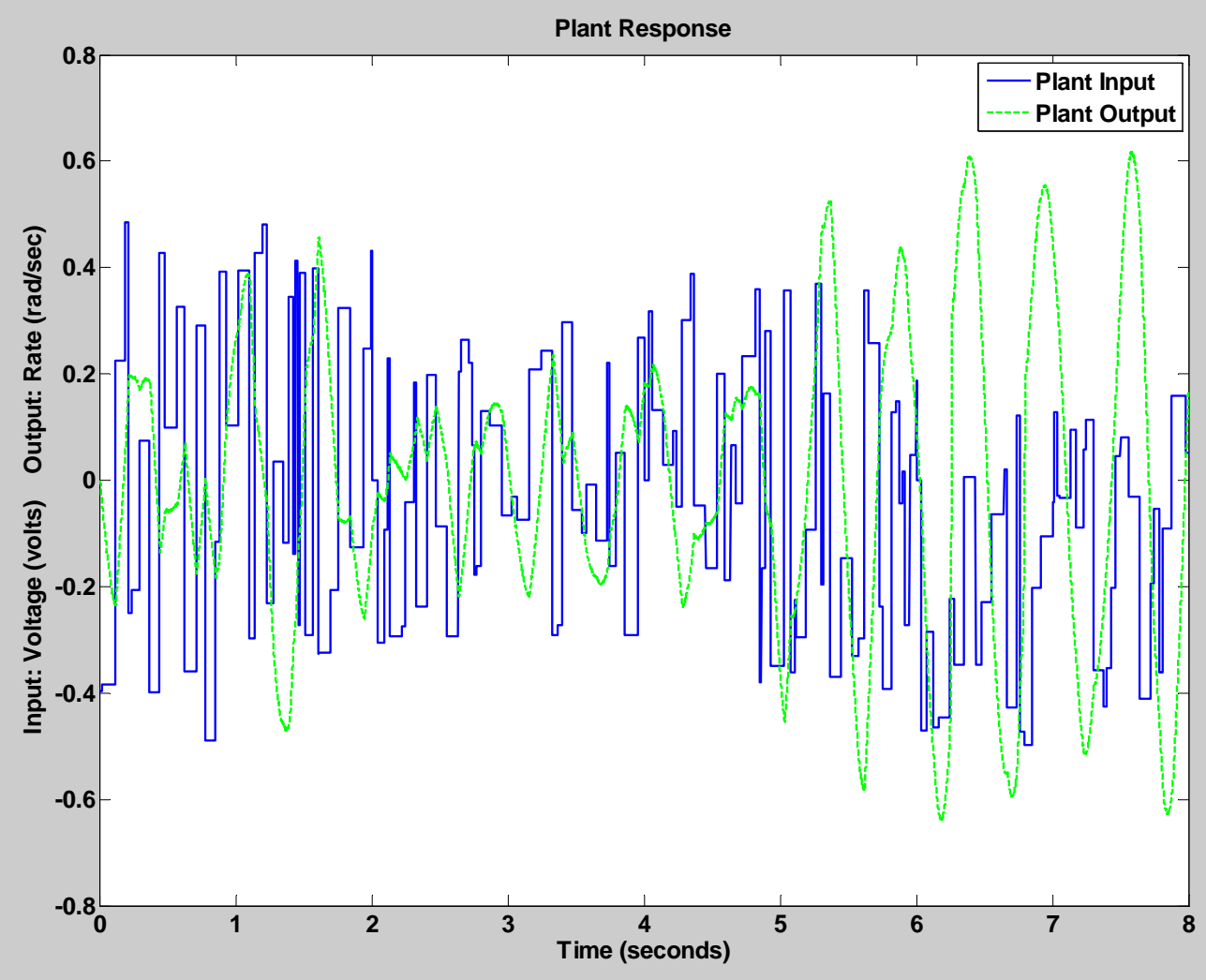

Figure 3.18: Plant Response: Random Signal (ARARI) (Section 3.3.5)

Figure 3.18 shows the response of the inner azimuth gimbal to the random signal described in Section 3.3.5. The sampling frequency for the signal was $f_{s}=2500 \mathrm{~Hz}$ and the total time duration was $t_{p}=8$ seconds. The expression for the signal is defined as follows:

$$
x(t)=\left\{\begin{array}{lr}
a_{1}+\left(b_{1}-a_{1}\right) \cdot \operatorname{rand}(1), & 0 \leq t<t_{1} \\
a_{m}+\left(b_{m}-a_{m}\right) \cdot \operatorname{rand}(1), & t_{m-1} \leq t<t_{m} \\
a_{p}+\left(b_{p}-a_{p}\right) \cdot \operatorname{rand}(1), & t_{p-1} \leq t \leq t_{p}
\end{array}\right.
$$

Where 


$$
\begin{gathered}
t_{m}=t_{m-1}+.01+(.1-.01) \cdot \operatorname{rand}(1) \\
\text { And } \\
a_{m}=\left\{\begin{array}{cc}
-0.5, & 0 \leq t<2 \\
-0.33, & 2 \leq t<4 \\
-0.4, & 4 \leq t<6 \\
-0.5, & 6 \leq t \leq 8
\end{array}\right.
\end{gathered}
$$

And

$$
b_{m}=\left\{\begin{array}{cc}
0.5, & 0 \leq t<2 \\
0.33, & 2 \leq t<4 \\
0.4, & 4 \leq t<6 \\
.167, & 6 \leq t \leq 8
\end{array}\right.
$$




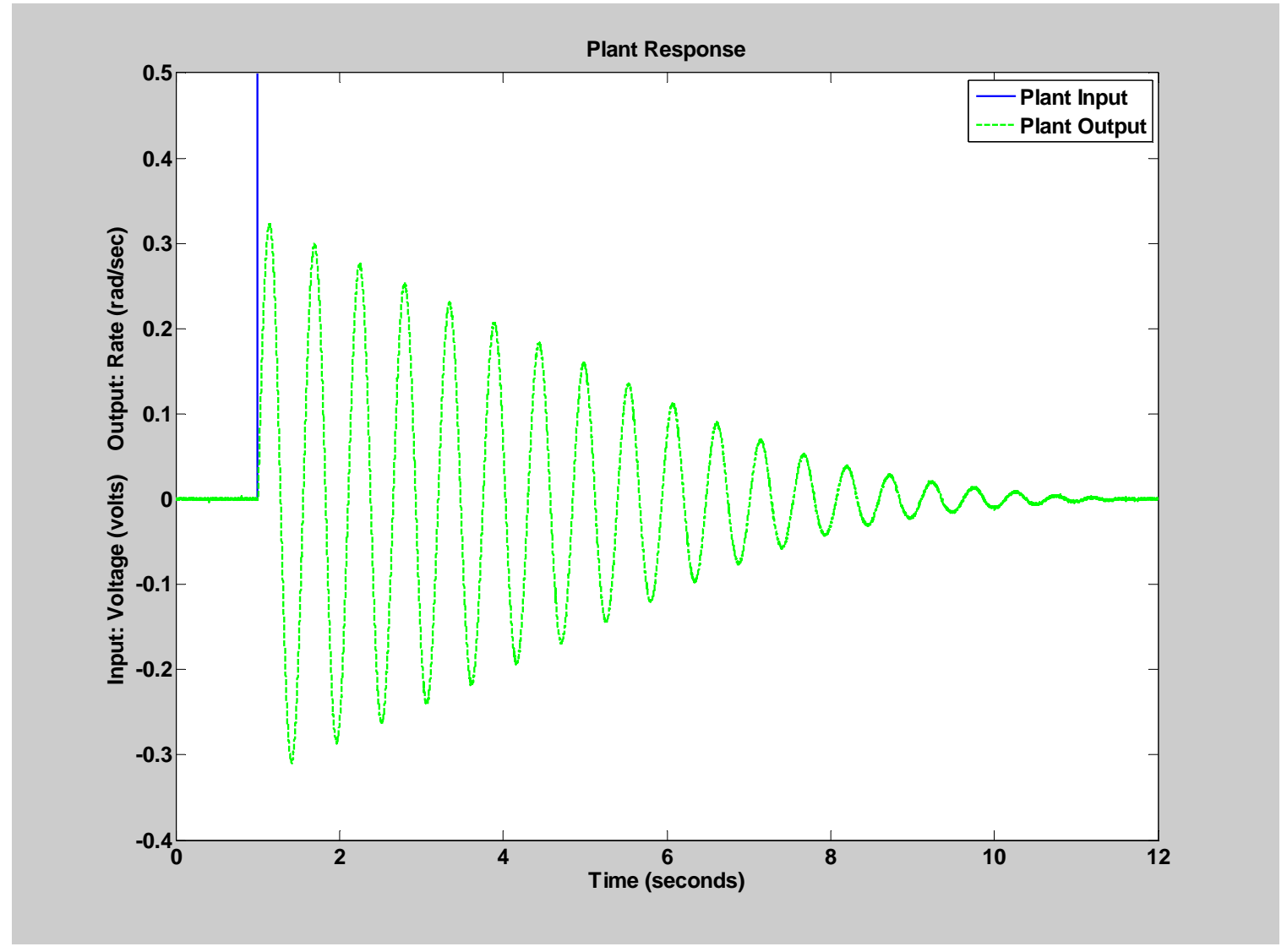

Figure 3.19: Plant Response: Step Function (Section 3.3.6)

Figure 3.19 shows the response of the plant (inner azimuth gimbal) due to a step function with amplitude of 0.5 volts. An amplitude of 0.5 was chosen based on experimental trial and error. At larger amplitudes the inner gimbal was reaching its limits in travel and distorted the data. The step function is described in section 3.3.6. The expression for the input voltage is defined as follows: $x(t)=\left\{\begin{array}{cl}0, & 0 \leq t<1 \\ 0.5, & 1 \leq t \leq 12\end{array}\right.$. The sampling frequency $f_{s}=2500 \mathrm{~Hz}$. 


\section{Chapter 4: Simulation Results}

This chapter presents the results obtained for a study of neural network modeling of the plant (inner azimuth gimbal) based on the data presented in Chapter 3. Included in this chapter is a study of the capabilities for the neural network to generalize, or the ability of the neural network to emulate the plant over a general operating range. The Levenberg-Marquardt (LM) algorithm is used to train the neural network because it has a high speed of divergence. The network is trained in batch mode (epoch mode) because networks tend to generalize better when trained in this fashion. The network weights are initialized using the Nguyen-Widrow method in order to expedite training time and increase the probability of finding a global minimum.

This chapter consists of four sections. The first section describes a software tool created in MATLAB in order to automate the training and simulation process. The second section focuses on finding the best network structure using one of the data sets given in Chapter 3. The third section presents the results when using this network structure to model each of the data sets given in Chapter 3. The last section presents the results for network generalization over all data sets.

\section{1 - Software Tool Development}

To speed the process of training and simulation of neural networks, a program was created in MATLAB. This program automates the training and simulation process. The program contains a graphical user interface (GUI) that can be utilized to import the 
data used for training, creates neural networks of several different structures, and initializes, trains, and simulates the neural networks using the imported data. In addition, the program can plot the imported data and the simulation results. The program's graphical user interface is shown in the figure below.

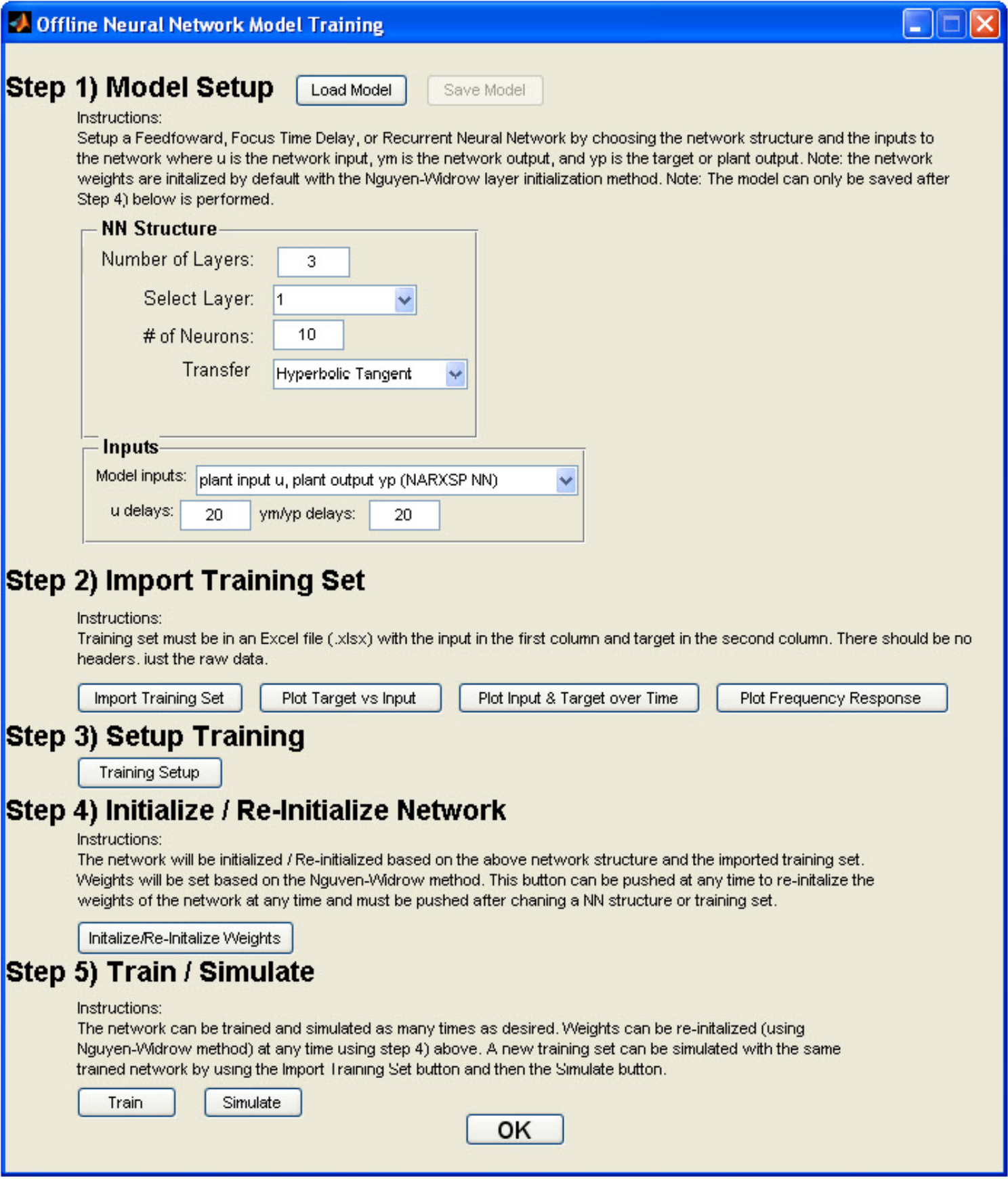

Figure 4.1: Program for Training \& Simulating Neural Networks 


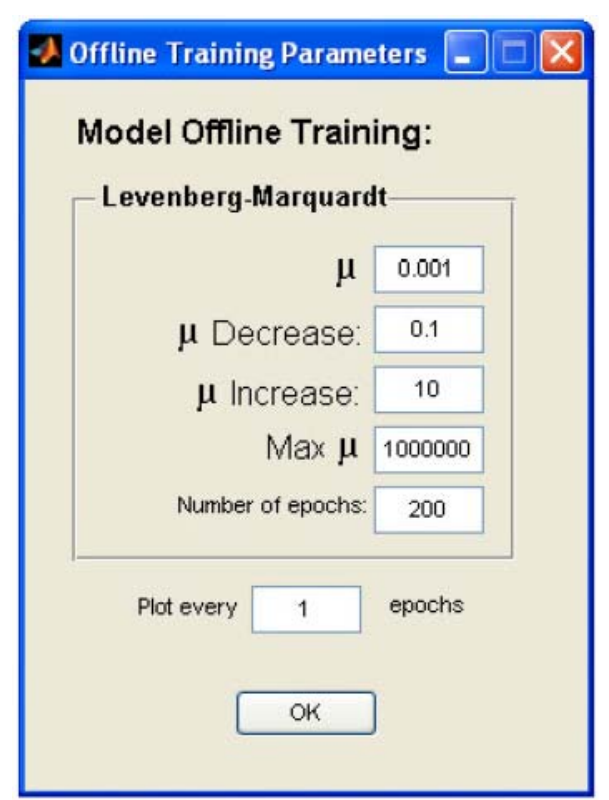

Figure 4.2: Training Setup for MATLAB Program

The GUI shown in Figure 4.1 contains two buttons at the top used for saving or loading neural networks. One of these buttons allows a trained or untrained neural network and all the current settings to to be saved to a file for later use. The other button can be used to load a previously saved neural network and settings.

Below these two buttons are inputs to setup the neural network structure. Four network structures can be created: (1) static feedforward; (2) the focused time-delay neural network (a standard feedforward network with a tapped-delay line on the input signal); (3) the nonlinear autoregressive network with exogenous inputs (NARX) (includes the tapped-delay line on the input signal, but adds feedback from the output of the network, which can also have a tapped-delay line) and; (4) the series parallel version of the nonlinear autoregressive network with exogenous inputs (NARXSP) (does not feedback the network output to the input but instead uses the network output target as a second input to the network). The latter three structures are dynamic. 
Any number of delays, layers and neurons can be chosen for the network. In addition, a different neuron transfer function (activation function) can be chosen for each layer. Three transfer functions can be chosen: linear, sigmoid, and hyperbolic tangent.

The row of buttons below the network structure inputs are used for importing the input/target data sets used to train the network. They also plot this data in both the time and frequency domain. The time-domain data can be plotted in two ways: target vs. input or target and input plotted vs. time.

Step 3 in Figure 4.2 displays a button that is used to bring up a window for setting the Levenberg-Marquardt (LM) algorithm training parameters. These parameters include: $\mu, \mu$ decrease, $\mu$ increase, maximum $\mu$, number of total epochs to train, and the interval between plot updates (in epochs). Note that the Levenberg-Marquardt algorithm is explained in Section 2.4.1. In this implementation of the algorithm, $\mu$ is decreased after each successful step and is increased only when a tentative step would increase the performance function. Step 4 in Figure 4.2 displays a button that is used to actually create the network and initialize the weights using the Nguyen-Widrow method as described in Section 2.3.5. Last, the two buttons at the bottom of the window under Step 5 in Figure 4.2 are used to start training and simulate the network using the last data set imported.

Using of the program to create and train a neural network can be summarized by following the steps: Step 1) - the network structure and inputs are set up; Step 2) - the data used for training is imported; Step 3) - the training parameters are set up; Step 4) the network is created; and Step 5) - the network is trained. As long as a network has been created, the weights can be re-initialized or network can be simulated at any time. Also, a data set can be plotted as long as a data set has been imported. The current 
trained or un-trained network can be simulated using a new data set by importing the data and pushing the "Simulate" button.

\section{2 - Network Structure Comparison, Training and Simulation Results}

Various neural network structures are compared by training to the $5 \mathrm{~Hz}$ sine wave data set described in Section 3.3.1 and Figure 3.2. Each structure is trained three times while re-initializing the weights each time. The networks are trained using the Levenberg-Marquardt algorithm which uses a batch method for training the network. As a reminder, with batch training (training by epoch) the network weights are not updated until after a complete set of input/target pairs have been presented to the network, where the complete set is considered one epoch. Note that there are 3500 training pairs in the training set for the $5 \mathrm{~Hz}$ sine wave mentioned above. This equates to a 1.4 second signal when the sampling rate is $2500 \mathrm{~Hz}$. After training each network structure through 200 iterations (200 epochs or weight updates) or 5 minutes, whichever comes first, the mean squared error (MSE) of the different structures is compared. The 5 minute time period refers to the time during which the computer trains the neural network. This value was chosen based on experimental data as it was discovered that the MSE for all network structures reached a relatively constant value within a 5 minute time period or before 200 epochs. The MSE is calculated by comparing the actual system output with the neural network output. The MSE is defined as: $M S E=\frac{\sum_{i=0}^{n} e(i)^{2}}{n}$ where $\mathrm{i}$ is the training pair index, $\mathrm{n}$ is the total number of training pairs (3500 for the $5 \mathrm{~Hz}$ sine wave) in the training set, and e is the error between the neural network output and the actual system output. In addition to comparing the MSE of different structures, an R-squared value (Coefficient of 
Determination) was calculated. The R-squared value is defined as the square of the Person Correlation coefficient $\mathrm{R}$. This value is obtained by dividing the covariance of the network output and real system output by the standard deviations of these two outputs multiplied. Thus, $R^{2}=\left(\frac{\operatorname{cov}(\hat{Y}, Y)}{\sigma_{\widehat{Y}} \sigma_{Y}}\right)^{2}=\left(\frac{E[(\hat{Y}-\bar{Y})(Y-\bar{Y})]}{\sigma_{\widehat{Y}} \sigma_{Y}}\right)^{2}$ where $\hat{Y}$ is the neural network output vector and $Y$ is the real system output vector. The R-squared value is used for comparison because it is a good measure of how well the model output fits the real system output or predicts future real system outputs. In other words, the value measures the agreement between the real output and the network output. The R-squared value can range between 0 and 1 . If the value is equal to 1 , it indicates that the model identifies the real system perfectly for the given training set. If the value equals 0 the two outputs are completely independent. The R-squared value is a commonly used measurement. For example, Microsoft Excel uses the value.

\subsubsection{Network Structure Description and Weight Initialization}

The networks are confined to having two layers; an input and output layer. Twolayer feed forward neural networks have been proven capable of approximating any arbitrary function [22]. The advantages of a two-layer network (one input and one output layer) include (1) rapid convergence, (2) computationally less expensive than a multilayer network, (3) no local minima, (4) network convergence is guaranteed, and (5) linear adaptive algorithms can be applied [2]. The disadvantages of a two layer network include (1) it is only applicable to small dimensional input space, (2) it is poor at generalizing the output training set, and (3) the training output layer may be ill-conditioned if the training set does not the span whole input space [2]. 
All networks in this study use the Hyperbolic Tangent Transfer function for neurons in the first layer and use the linear transfer function for neurons in the output layer. The Hyperbolic Tangent function is defined as $y(v(n))=\frac{e^{v(n)}-e^{-v(n)}}{e^{v(n)}+e^{-v(n)}}$ where $\mathrm{n}$ is training pair index (or signal time sample). This function is used because it is fairly linear in the active region and has a finite active input range. This allows weight initialization using the Nguyen-Widrow method (described in more detail below). This function is also used because it allows the network to learn non-linear relationships. The linear function is defined as $y(v(n))=v(n)$ and is used for the output layer of the network. This function is used because it allows the network to scale to any magnitude target by changing the weights between input and output layer.

Four different network input arrangements or structure types are used in this study. The first of these is the standard Feedforward, as mentioned in chapter 2 and shown in Figure 2.4. The second network structure used is called the Focus Time-Delay Line Neural Network (FTDNN) or Focused Time Lagged Feedforward as described in chapter 2 and shown in Figure 2.6. This structure includes a Tapped Delay Line (TDL) on the input to the network. The third network structure is called a NonLinear Autogressive Network with Exogenous Inputs (NARX) and is a Recurrent network. In this type of structure, the network output is fed back as a second input. As with the FTDNN network, a TDL occurs at the input and in this case two TDL occur (one for each input). Recurrent networks are described in chapter 2 and an example is shown in Figure 2.5. The fourth and last network structure is a Series-Parallel Nonlinear Autogressive network (NARXSP). This structure is similar to the NARX, except that instead of the network output being fed back as a second input, the network target is used as the second 
input. Again, both inputs consist of TDLs. In order to generate the above networks the Matlab NN Toolbox (Version 6) is utilized. To create the Static FeedForward and FTDNN networks the newfftd() Matlab function is used. The NARX network is created using the newnarx() function and the NARXSP network is created using the newnarxsp() function.

The weights and biases (bias weights) of layer 1 for all network structures throughout this study are initialized using the Nguyen-Widrow method (introduced in section 2.3.5). The weights and bias weights for the output layer of the network are initialized using the Matlab function rands(). The rands() function initializes the weights to a uniformly distributed pseudorandom value between -1 and 1 . The Nguyen-Widrow method is used for the purpose of decreasing training time.

The basis for the Nguyen-Widrow method rely on how well a two-layer neural network can approximate any nonlinear function by forming a union of piece-wise linear segments [22]. Each of these segments is attributed by a neuron in the first layer of the network. In this method the weights of the first layer (of the 2-layer network) are chosen in order to distribute the active region of each neuron in the layer evenly across the layer's input space [7]. Note that the input space is defined as the range of input values for that layer. The active region referred to above equals -1 to 1 for a neuron using the Hyperbolic Tangent function. The Nguyen-Widrow method is used in this study because this method has been shown to achieve major improvements in training time over a large number of different training problems [22].

In order to implement the algorithm the Matlab Neural Network Toolbox (Version 6) function is used and is called by default when generating the network 
structures mentioned above. The steps to implement the weight initialization are as follows: First, the weights in layer one are uniformly distributed randomly between -1 and 1 using the randnr() Matlab function. Next, the magnitude of the weight vector $W_{i}$ for each neuron $\mathrm{i}$ is scaled using the following formula: $\left|W_{i}\right|=0.7 H^{\frac{1}{N}}$, where $\mathrm{H}$ is the number of neurons in layer 1 , and $\mathrm{N}$ is the number of elements in vector $\mathrm{X}$ or vector $W_{i}$. Lastly, the bias weights of the layer are set equal to a uniform random number between $-\left|W_{i}\right|$ and $\left|W_{i}\right|$. It is important to note that for the above equation to work the range of the input vector $\mathrm{X}$ must be -1 to 1 . Therefore, when the network is simulated and trained the input vector $\mathrm{X}$ is scaled between these two values.

\subsubsection{Training Method}

All training in this study is accomplished by batch training using the LevenbergMarquardt algorithm (explained in Section 2.4.1). To implement the training the Matlab Neural Network Toolbox fuction trainlm() was used. The method and algorithm used for this training is described below and in [21]. In the Levenberg-Marquardt algorithm (as defined for batch training), the weights are updated using the expression $\Delta \boldsymbol{w}=$ $-\left(J^{T}(\boldsymbol{w}) J(\boldsymbol{w})+\mu I\right)^{-1} J^{T}(\boldsymbol{w}) \boldsymbol{e}(\boldsymbol{w})$ where $\boldsymbol{w}$ is a weight vector containing all the weights in the network and is defined as $\boldsymbol{w}(i)=\left[w_{1}(i), w_{2}(i), \ldots, w_{m}(i)\right]^{T}$. In this equation $\mathrm{m}$ is the total number of weights in the network, $J$ symbolizes the Jacobian matrix, $\mu$ is a small positive value, and I is the identity matrix. In addition, $\boldsymbol{e}(\boldsymbol{w})=[e(1), e(2), \ldots, e(N)]^{T}$ is a vector of network output errors where $\mathrm{N}=\mathrm{Q} \times \mathrm{J}$. The value $\mathrm{Q}$ is the total number of training pairs in the training set and $\mathrm{J}$ is the number of network outputs. For batch training the Jacobian matrix is defined as: 


$$
J(\boldsymbol{w})=\left[\begin{array}{cccc}
\frac{\partial e(1)}{\partial w_{1}} & \frac{\partial e(1)}{\partial w_{2}} & \cdots & \frac{\partial e(1)}{\partial w_{m}} \\
\frac{\partial e(2)}{\partial w_{1}} & \frac{\partial e(2)}{\partial w_{2}} & \cdots & \frac{\partial e(2)}{\partial w_{m}} \\
\vdots & \vdots & & \vdots \\
\frac{\partial e(N)}{\partial w_{1}} & \frac{\partial e(N)}{\partial w_{2}} & \cdots & \frac{\partial e(N)}{\partial w_{m}}
\end{array}\right]
$$

As mentioned in Chapter 2, the terms in $J(n)$ can be found by calculating the network gradients using the standard backpropagation algorithm with one modification at the output layer: the output layer local gradient is defined as $\delta_{j}^{L M}=-f_{j}^{\prime}\left(v_{j}(n)\right)$, where $\mathrm{f}$ is the activation function for output neuron $\mathrm{j}$. Using this modified local gradient $\delta_{j}^{L M}$ the terms in the Jacobian matrix for the output layer are defined as $\frac{\partial e}{\partial w_{j i}}=\delta_{j}^{L M} x_{i}(n)$ where $x_{i}(n)$ is the input to weight $w_{j i}$ between output neuron $\mathrm{j}$ and neuron $\mathrm{i}$ in the previous layer. The terms for hidden layer weights can be calculated in a similar fashion by backpropagating the local gradient through the network using Equation 36.

Again, note that the performance function for this algorithm is $\varepsilon(\boldsymbol{w})=$ $\sum_{i=1}^{N} e_{i}^{2}(w)$ where $\mathrm{N}$ is the total number of training pairs for a network with one output and the gradient for this performance fuction is equal to $J^{T}(n) \boldsymbol{e}(n)$ (as seen in the above expression).

The constant $\mu$ in the expression for $\Delta \boldsymbol{w}$ above is made to be adaptive using the following equation:

$$
\mu(n+1)=\left\{\begin{array}{lr}
\mu(n) \beta, & \varepsilon(n)>\varepsilon(n-1) \\
\mu(n) \alpha, & \varepsilon(n)<\varepsilon(n-1) \\
\mu(n), & \mu(n+1)>\mu \max
\end{array}\right.
$$

The training parameters are set as follows: the initial value for $\mu=0.001, \alpha=0.1$, $\beta=10, \mu \max =1 E 10$, and the number of epochs to train is 200 . The above expression allows the network to be trained in a Gauss-Newton type fashion as the performance 
measure reaches a minimum. The value for $\mu$ decreases whenever this performance measure $\varepsilon(n)$ decreases and is increased whenever a step would increase the performance function. If $\mu$ becomes larger then $\mu \max$ the algorithm is stopped. In addition to the above parameters the MSE is set to be plotted every epoch.

In summary, the Marquardt algorithm proceeds as follows: 1) All inputs are presented to the network and the corresponding outputs and errors are calculated. The sum of squares of errors is computed over all inputs. 2) The Jacobian matrix is calculated using $\frac{\partial e}{\partial w_{j i}}=\delta_{j}{ }^{L M}(n) x_{i}(n)$ where $\delta_{j}{ }^{L M}(n)=-f_{j}^{\prime}\left(v_{j}(n)\right)$ for the output layer and $\delta_{k}{ }^{L M}(n)=f_{k}^{\prime}\left(v_{k}(n)\right) \sum_{j=1}^{J} \delta_{j}{ }^{L M}(n) w_{j k}(n)$ (reference Equation 36) for a hidden neuron layer. 3) The value for $\Delta \boldsymbol{w}$ is calculated as $\left.\Delta \boldsymbol{w}=-\left(J^{T}(\boldsymbol{w}) J(\boldsymbol{w})+\mu I\right)^{-1} J^{T}(\boldsymbol{w}) \boldsymbol{e}(\boldsymbol{w}) .4\right)$ The sum of squares of errors is re-computed using new weights $\boldsymbol{w}+\Delta \boldsymbol{w}$. If the new sum of squares is smaller than what was computed in step one, then $\mu$ is multiplied by $\alpha, \boldsymbol{w}$ is set equal to $\boldsymbol{w}+\Delta \boldsymbol{w}$ and step 1 through 3 is repeated. If the sum of squares is not reduced then $\mu$ is multiplied by $\beta$ and steps 3 through 4 are repeated.

All of the training and simulations in this study were accomplished using the same personal computer. The computer had an AMD Athlon 64 X2 Duel Core Processor $3800+2.01 \mathrm{GHz}$ and $1 \mathrm{~GB}$ of ram.

\subsubsection{Training and Simulation Results}

The first neural network structure studied was a standard Feedforward with 5 neurons in the hidden layer, and one neuron in the output layer. The network was created using the Matlab function newfftd() with the number of delays on the input set to zero. 
As a reminder, the Nguyen-Widrow method is used for weight initialization, the Hyperbolic Tangent function is used for the hidden layer and the Linear activation function is used for the output layer. The Levenberg-Marquardt algorithm is used with batch training. A block diagram of the network is shown in Figure 4.3 below. The network was trained through three trials (200 epochs each). Before each trial the network weights were re-initialized. All three trials produced the same mean squared error: 0.014151. The R-squared value was also the same for all three trials was equal to 0.0028621 . This R-squared value is relatively close to zero thus indicating this neural network structure does not model the system very well. A Plot of the mean squared error vs. epoch for the last trial and the simulation results are shown in Figure 4.4 through Figure 4.6 below.

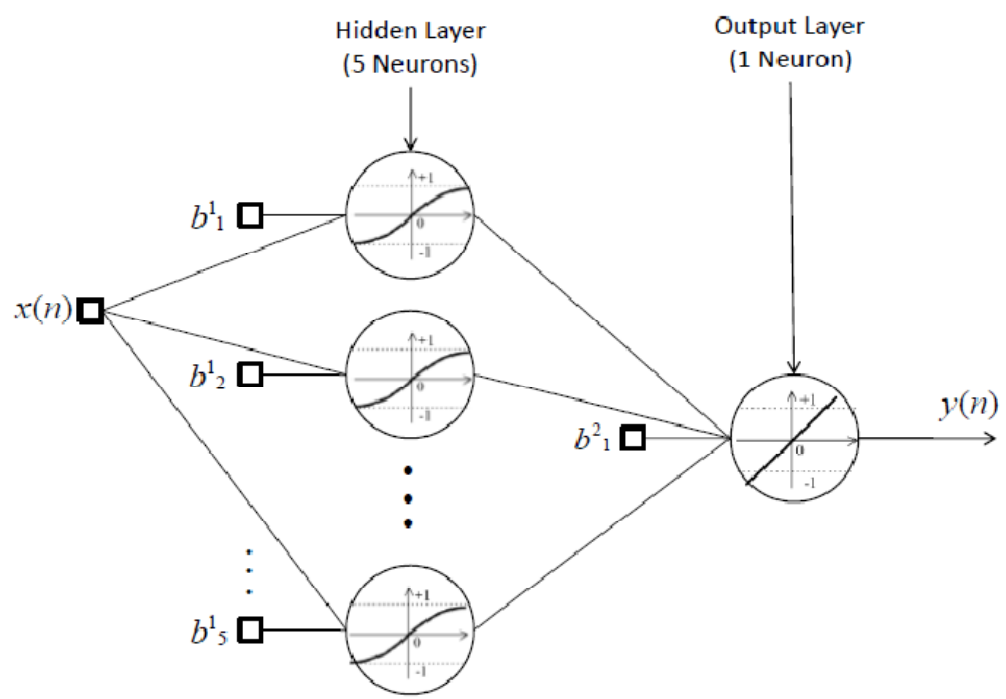

Figure 4.3: Network Structure (Standard Feedforward) 


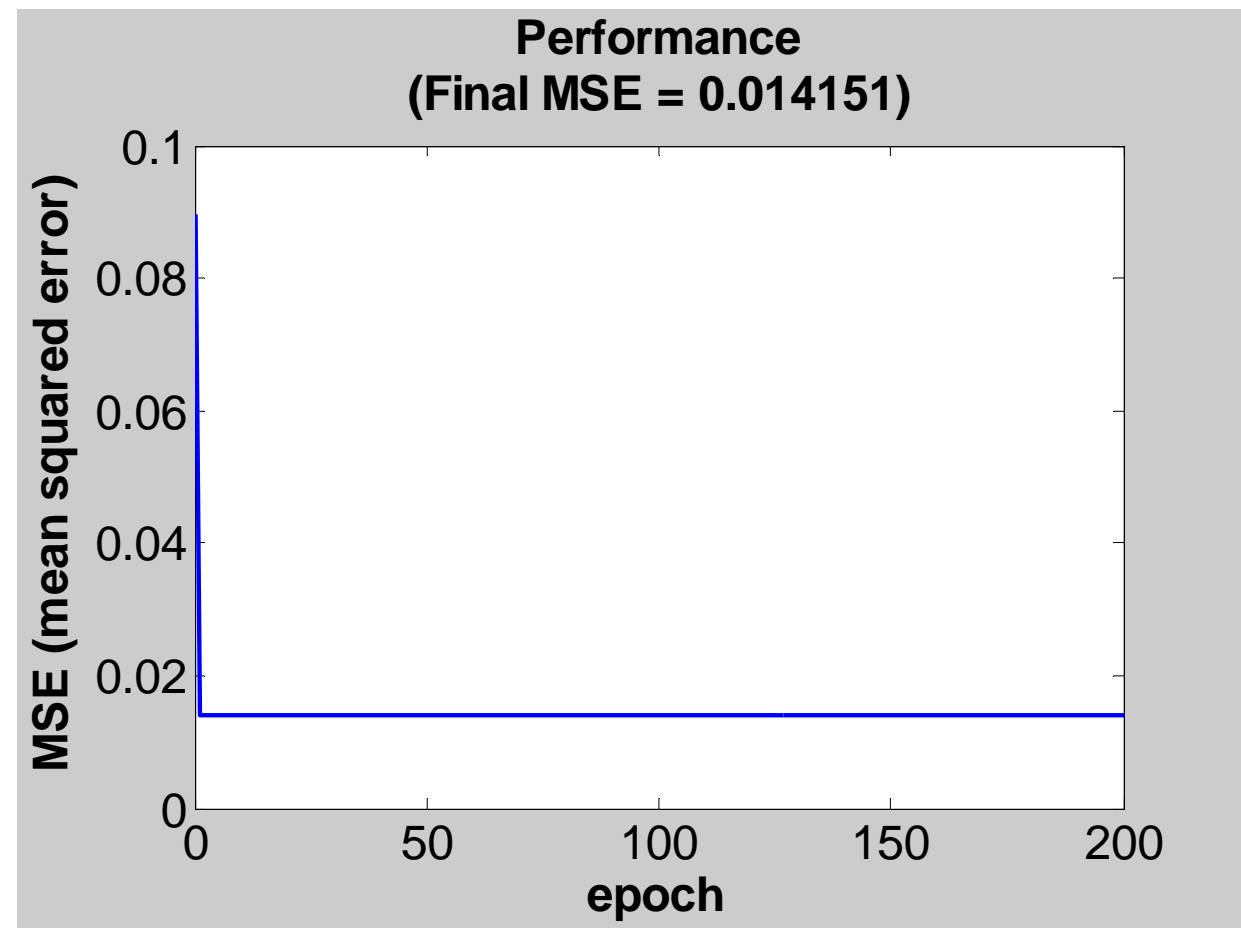

Figure 4.4: Performance Plot (Standard Feedforward NN)

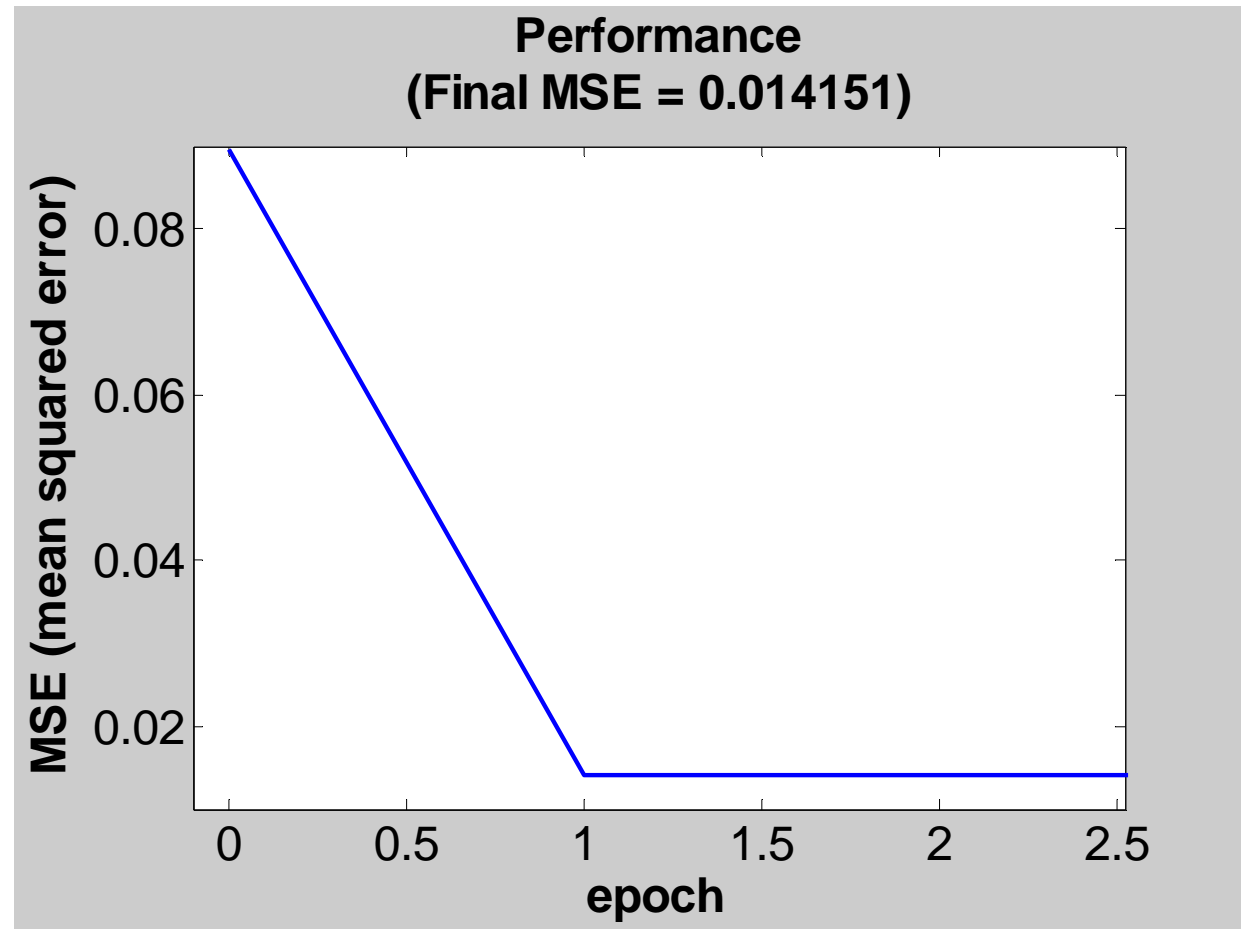

Figure 4.5: Performance Zoomed (Standard Feedforward NN) 
(Sampling Frequency fs $=\mathbf{2 5 0 0 ~ H z}$ )

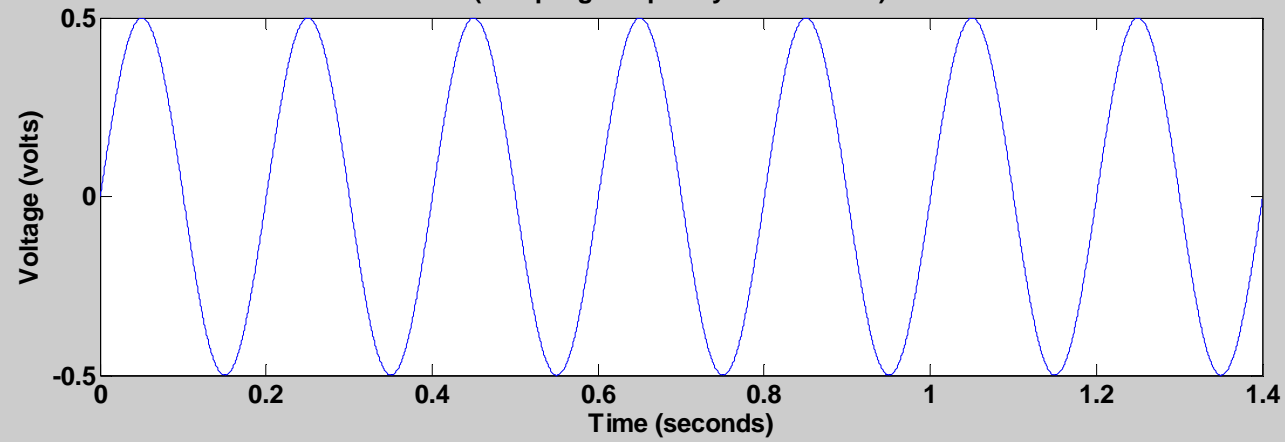

Neural Network Output \& Plant Output (Target)

( MSE $=0.014151$ R-Squared $=0.0028621$ )

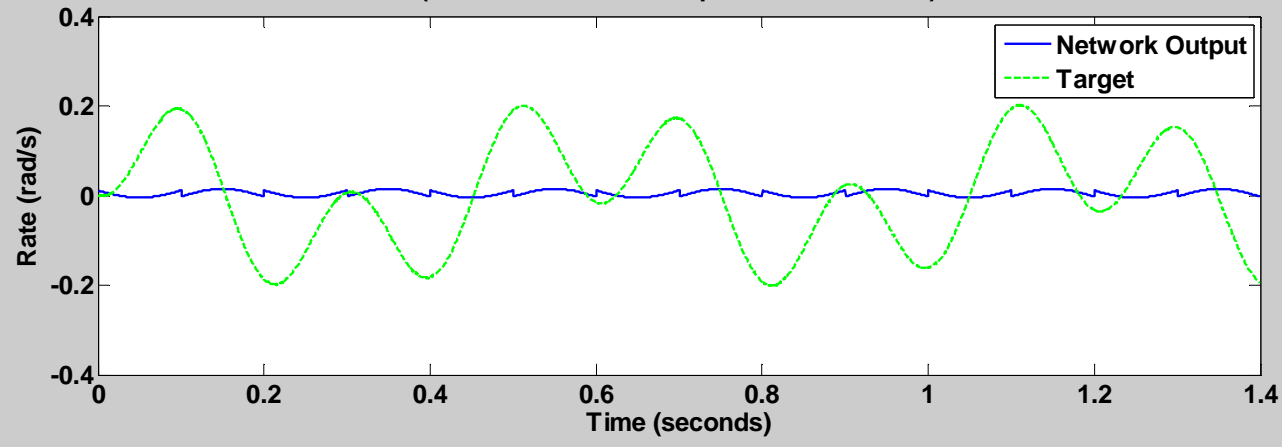

Figure 4.6: Simulation (Standard FF Structure) 
The next network structure was a focus time-delay line neural network (FTDNN). The network also had 5 neurons in the hidden layer, but had a tapped delay line on the input consisting of 5 delays. The network was also created using the Matlab function newfftd(), but with five input delays. Again, the Nguyen-Widrow method is used for weight initialization, the hyperbolic Tangent function is used for the hidden layer and the Linear activation function for the output layer. The Levenberg-Marquardt algorithm is used for training. The network structure is shown in Figure 4.7 below. Note that the value $\mathrm{n}$ is the training pair index or time sample and ranges between 1 and 3500 for the $5 \mathrm{~Hz}$ sine wave training set. After 200 epochs the MSE for the three trials (weights reinitialized before each trial) equaled $0.0059231, .005923,0.0059257$. The R-squared values were $0.58264, .58265,0.58245$. A plot of the mean squared error vs. epoch and the simulation results for the last trial are shown in Figure 4.8 through Figure 4.10. The network that produced the lowest MSE was simulated and the results are shown below.

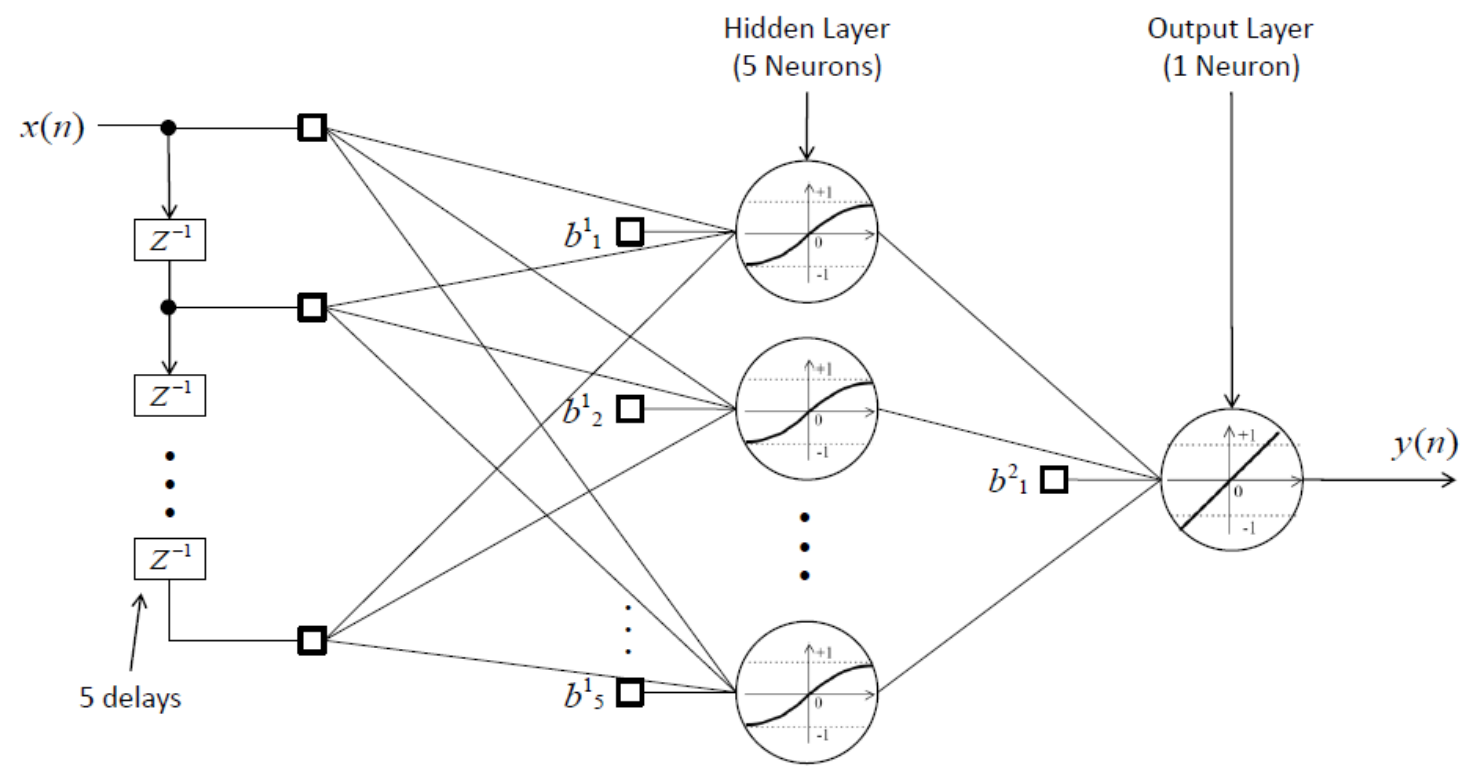

Figure 4.7: Network Structure (FTDNN) 


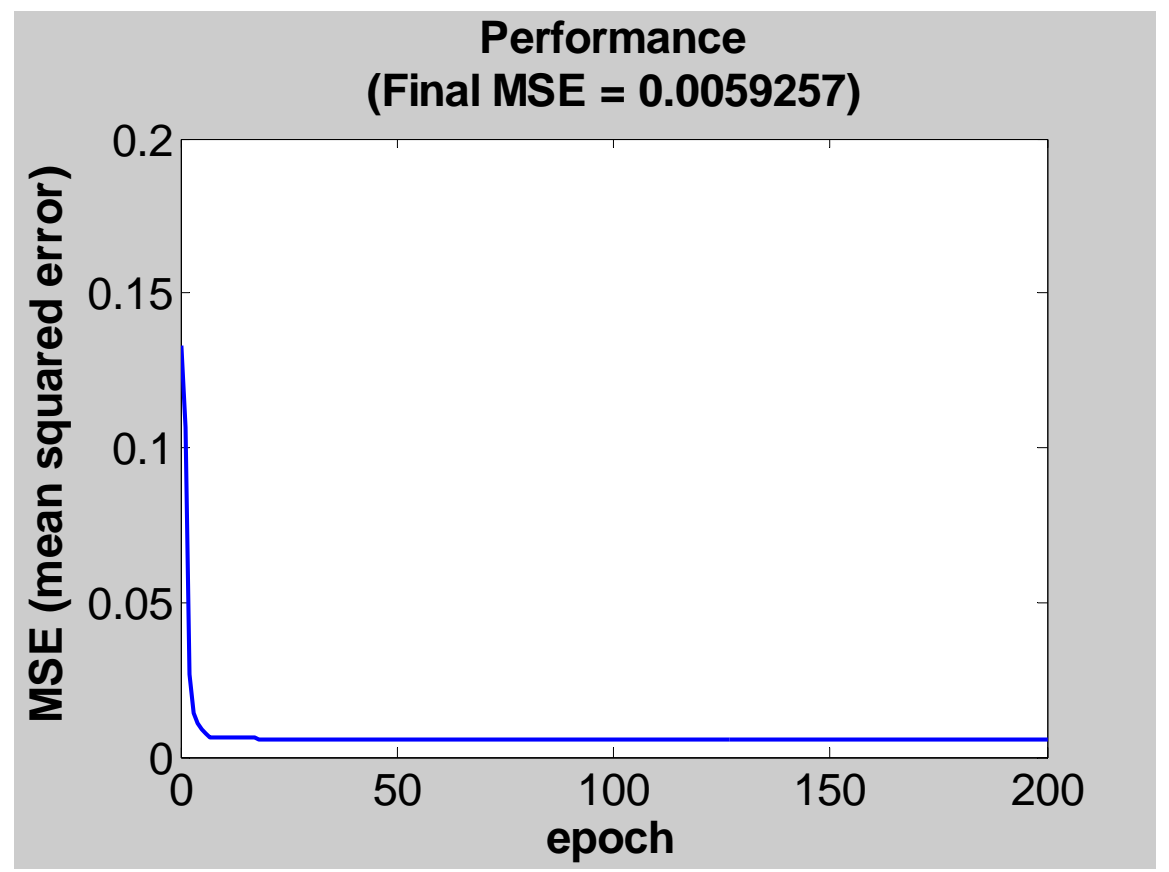

Figure 4.8: Performance (FTDNN Structure)

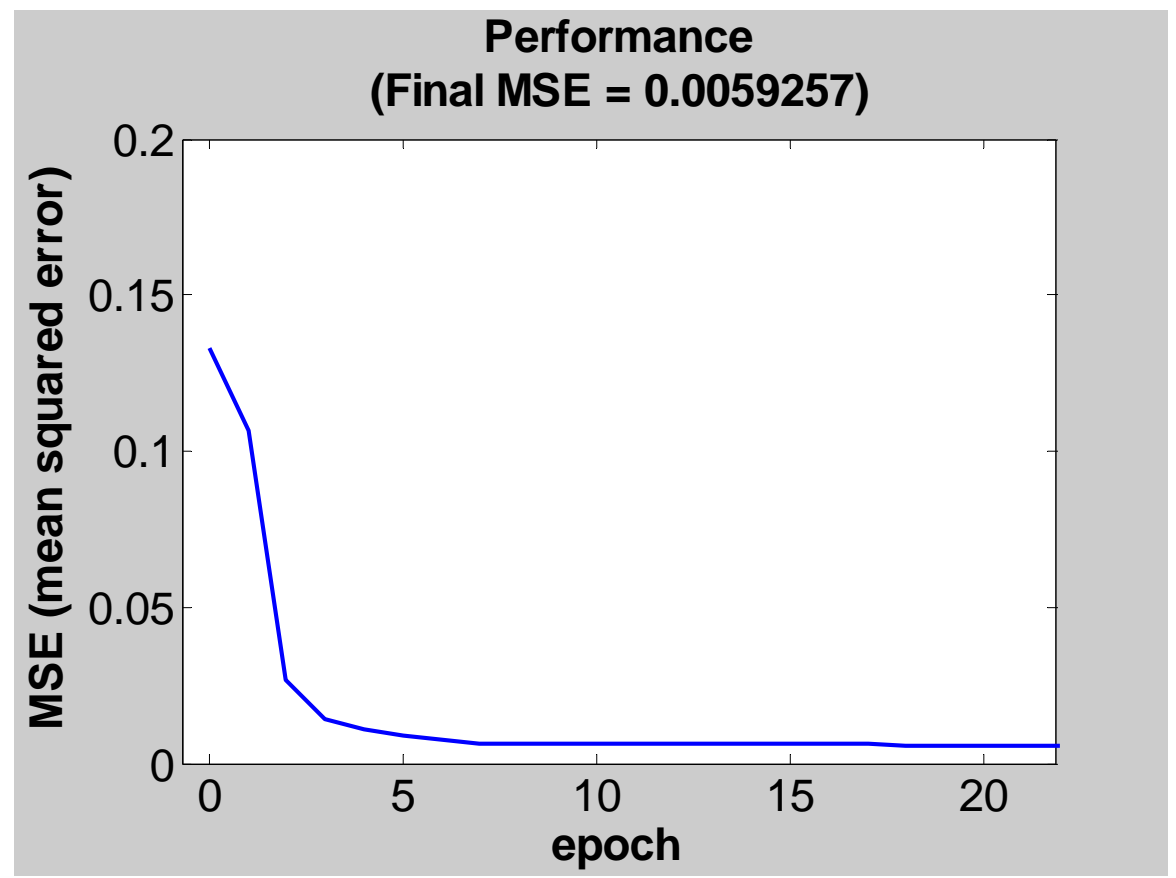

Figure 4.9: Performance Zoomed (FTDNN Structure) 

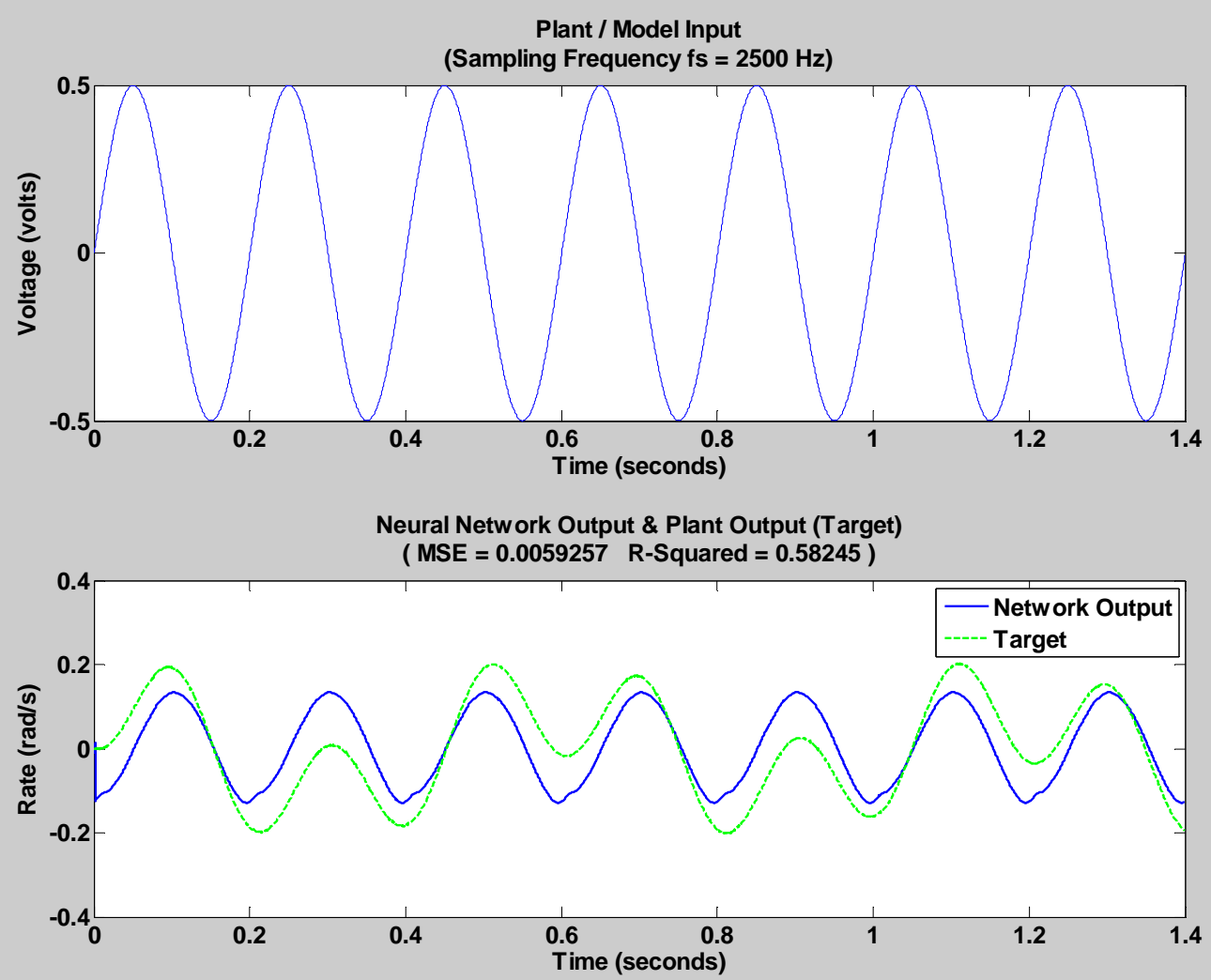

Figure 4.10: Simulation (FTDNN Structure)

The next network structure was a Nonlinear Autoregressive Network with Exogenous Inputs (NARX). The network again had 5 neurons in the first layer and 1 output neuron. The networks output was fed back as a second input and both inputs included a 5 delay tapped delay line. The neural network was created using the Matlab function newnarx () . The network structure is shown in the Figure 4.11 below. The training was stopped after five minutes for all three trials. The mean squared error for trials one through three was $0.0057619, .0086563, .0058255$, respectively, and the number of epochs reached after the 5 minutes was 14,12 , and 13 respectively. The Rsquared values for the three trails were $.59419, .40543, .58954$. A plot of the mean squared error vs. epoch and the simulation results for the first trial is shown in Figure 4.12 and Figure 4.13 below. 


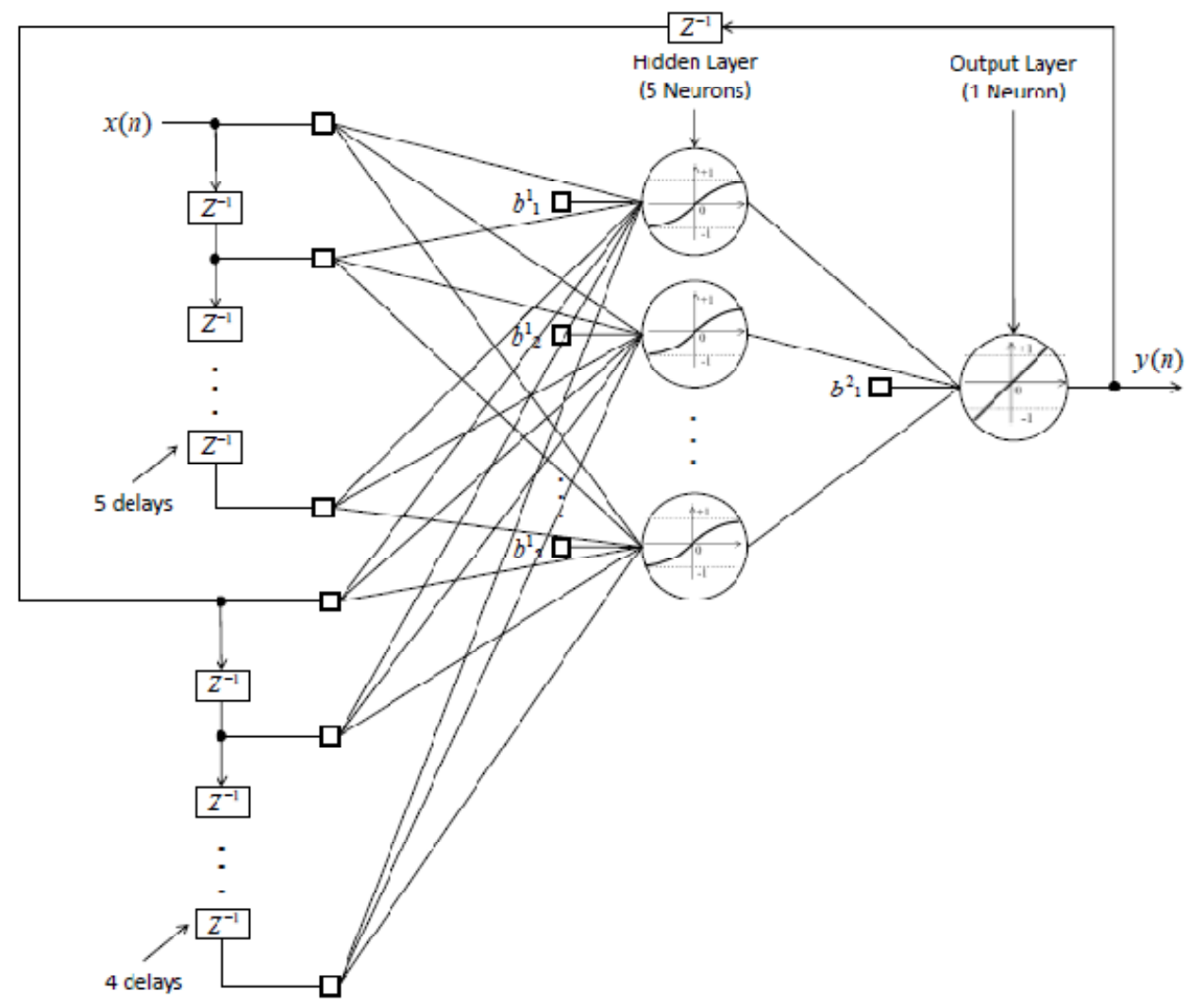

Figure 4.11: Network Structure (NARX)

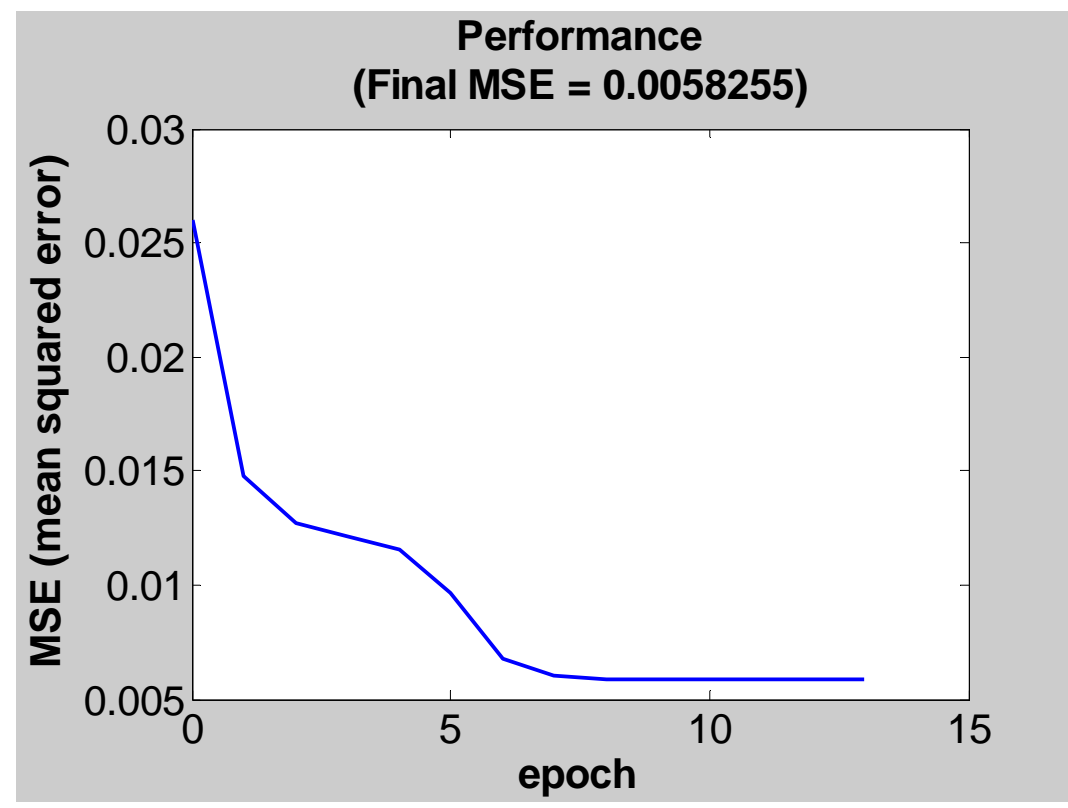

Figure 4.12: Performance (NARX Structure) 


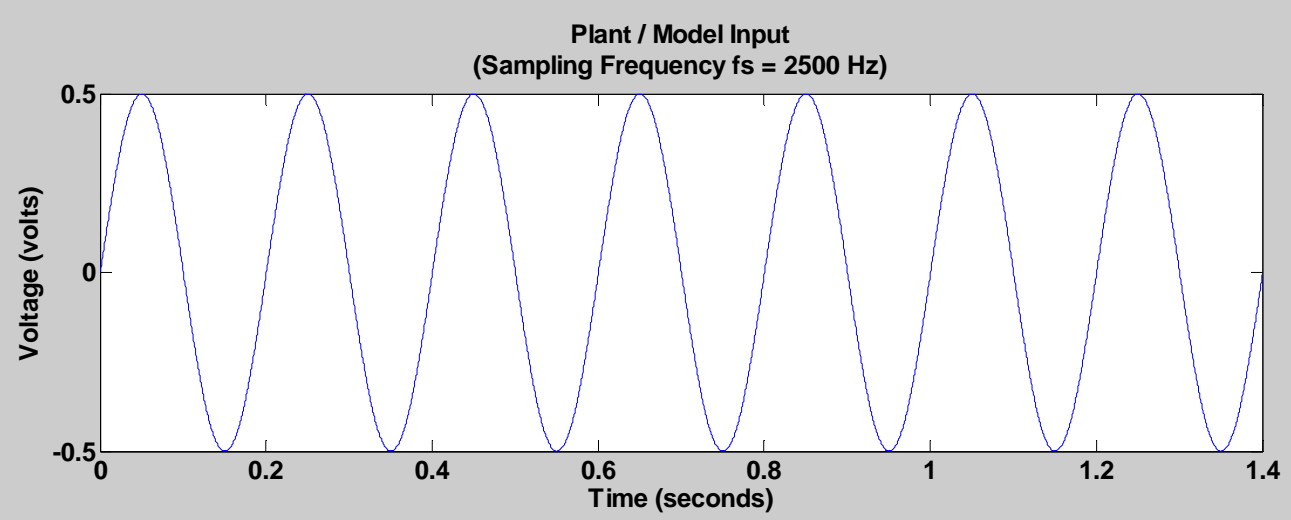

Neural Network Output \& Plant Output (Target)

( MSE $=0.0058255$ R-Squared $=0.58954$ )

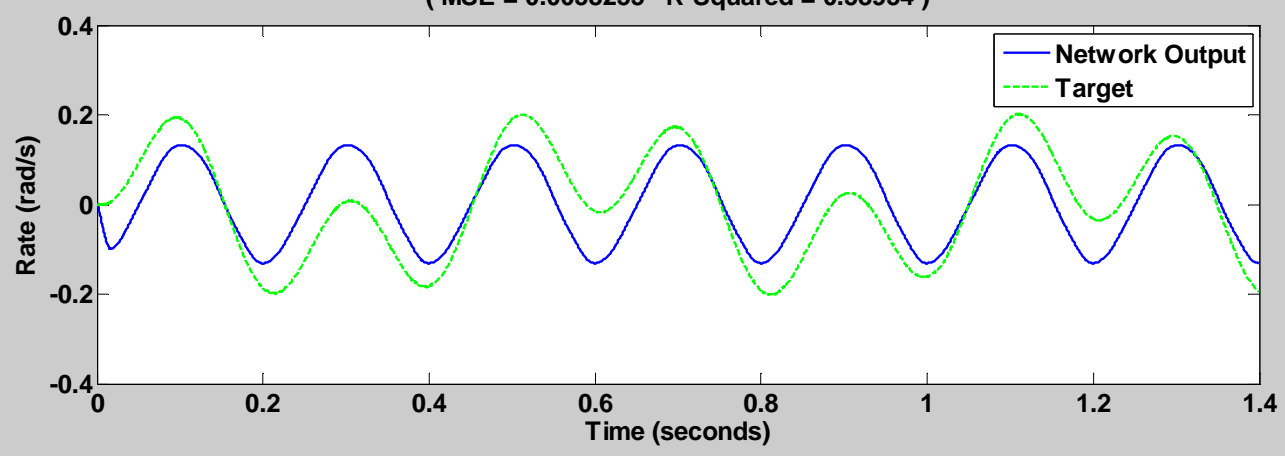

Figure 4.13: Simulation (NARX Structure)

The next network structure was the Serial-Parallel Nonlinear Autoregressive Network with Exogenous Inputs (NARXSP). The network had 2 layers; a 5 neuron hidden layer and a 1 neuron output layer. Again, a tapped delay line with five delays was placed on the input. However, instead of the network output being fed back to the input the networks target $\mathrm{d}$ is fed as a second input with a 5 delay tapped delay line. The basic block diagram of the network is shown in Figure 4.14 below. After 200 epochs the three trials produced mean squared errors of 1.3702E-7, 1.4432E-7, 1.4169E-7 respectively. The R-squared values for all three trails was .99999. A plot of the mean squared error vs. epoch and the simulation results for the last trial are shown in Figure 4.15 and Figure 4.17 below. 


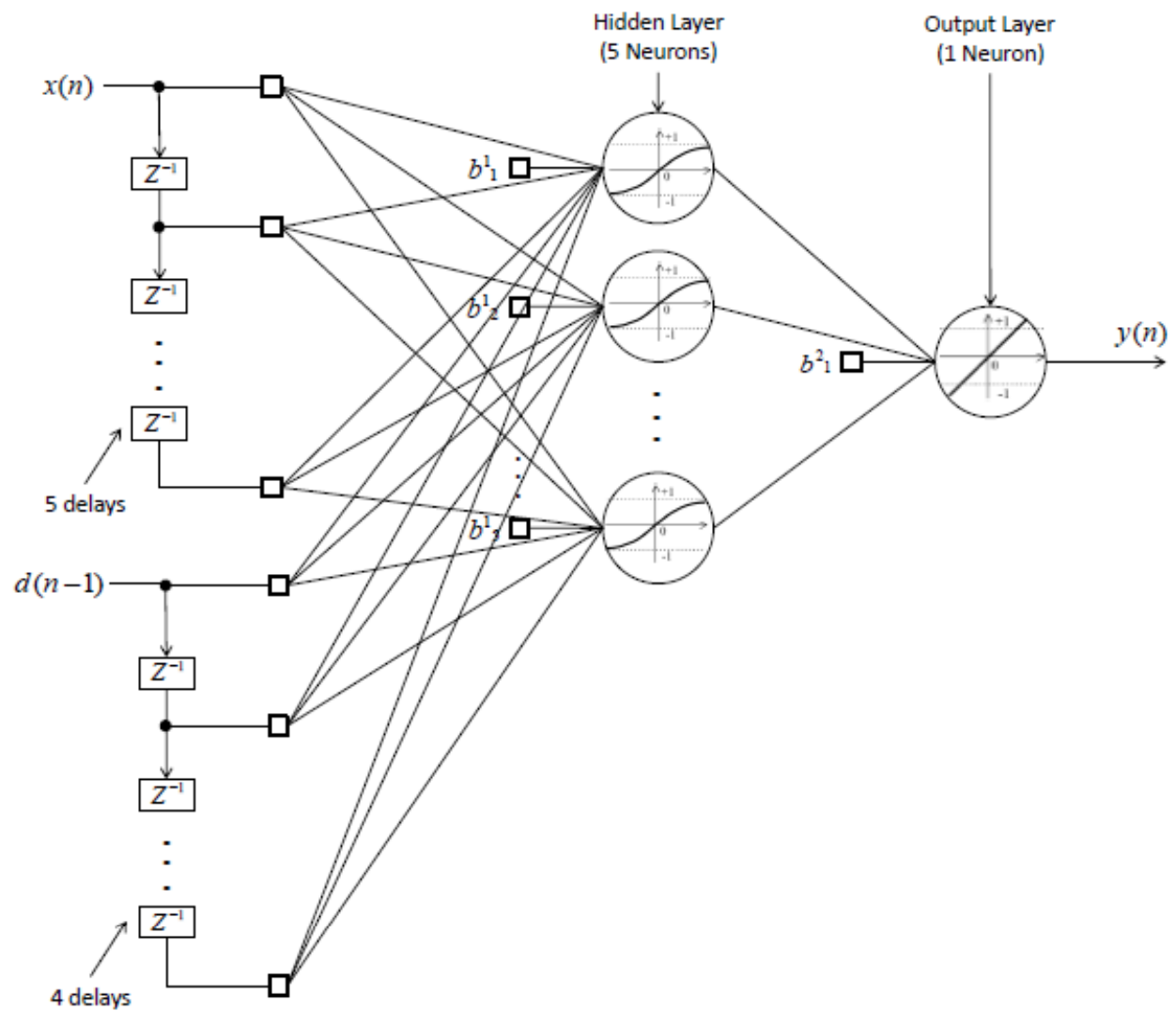

Figure 4.14: NARXSP Network Structure

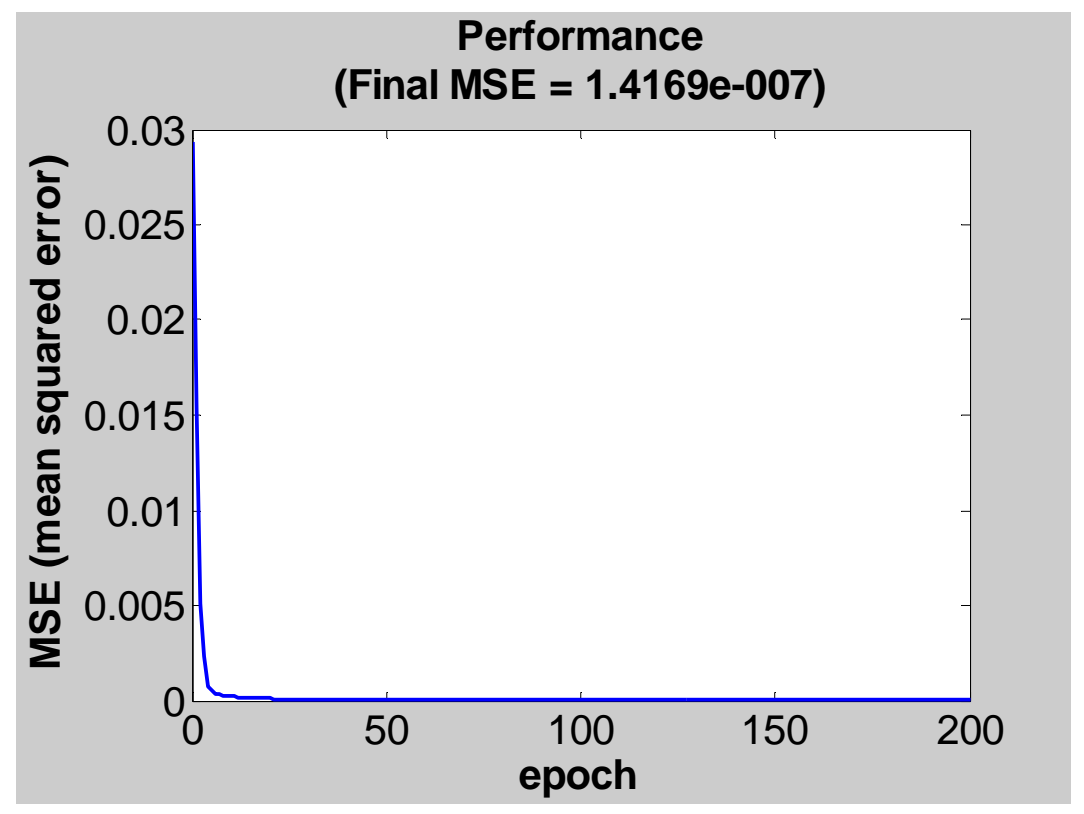

Figure 4.15: Performance (NARXSP w/ 5 neurons) 


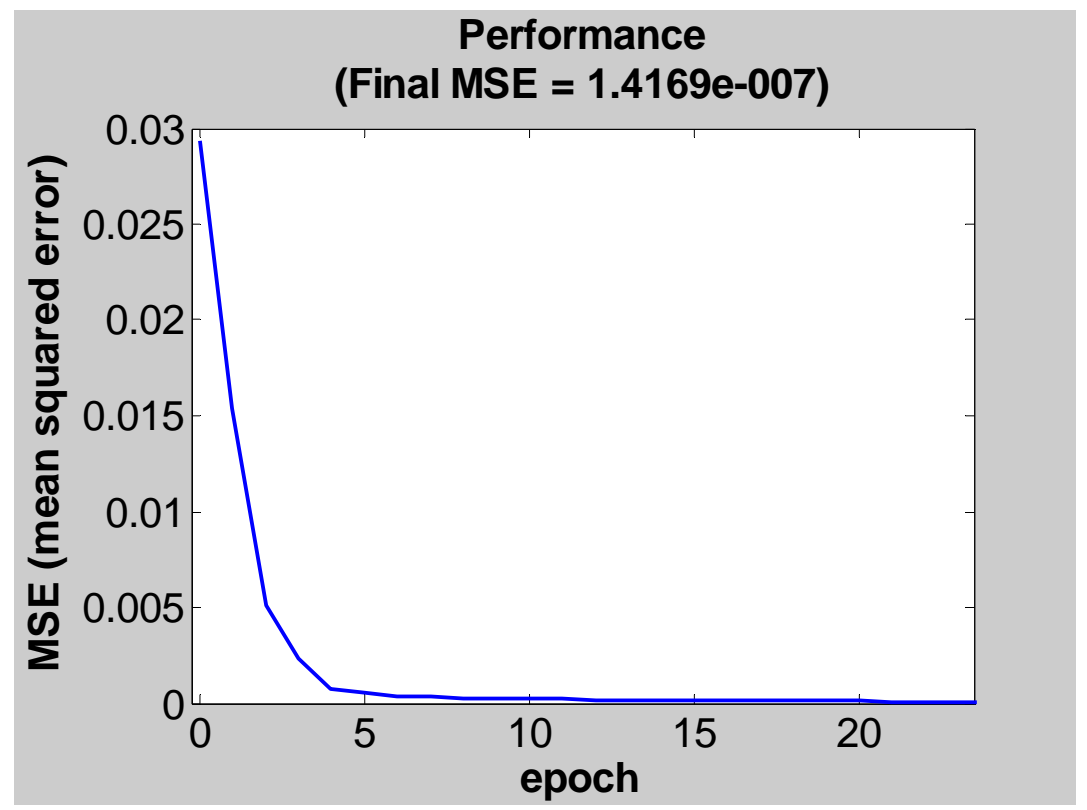

Figure 4.16: Performance (NARXSP w/ 5 neurons) Zoomed
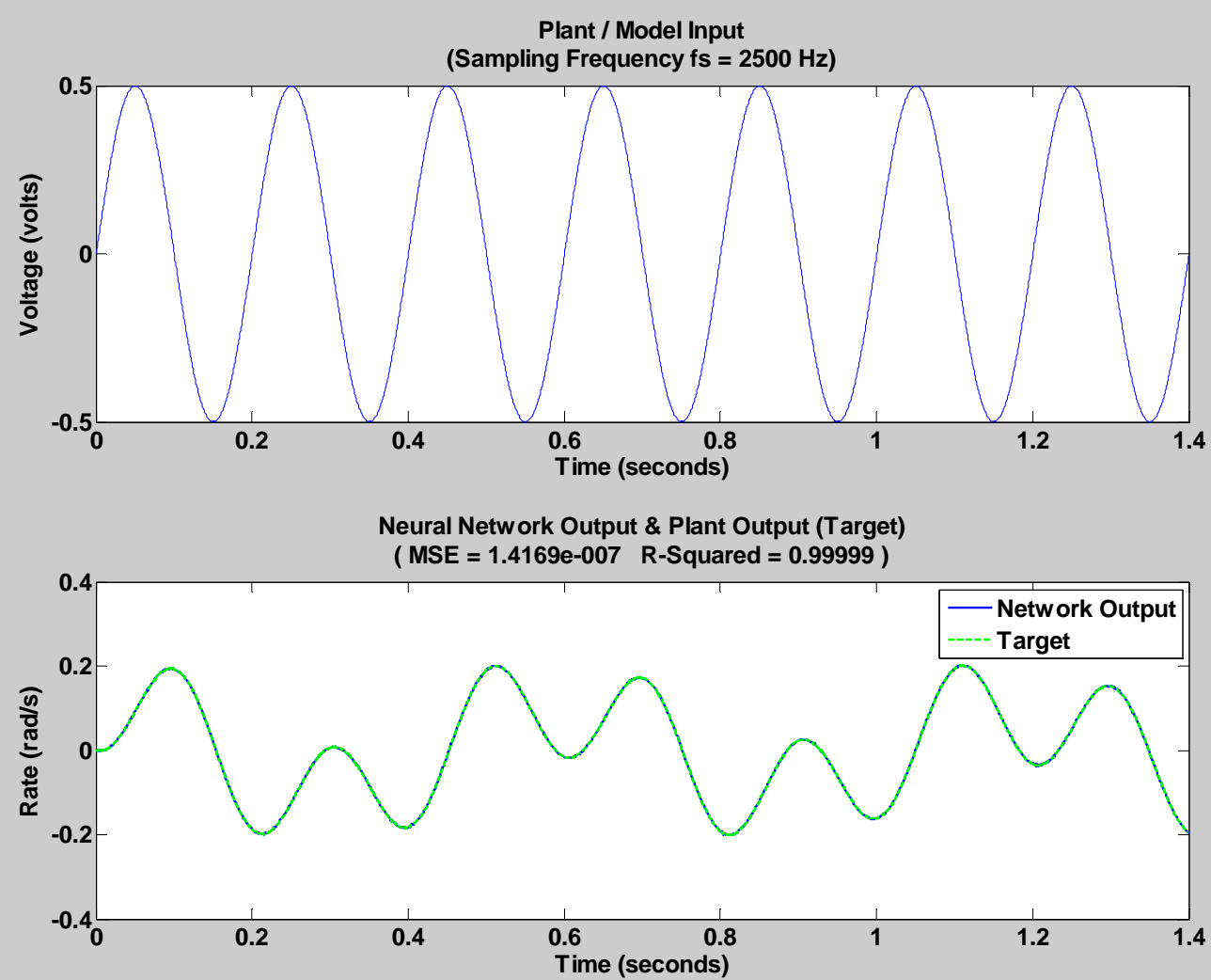

Figure 4.17: Simulation (NARXSP Structure w/ 5 neurons) 
Three more NARXSP network structures were tested. The transfer functions, number of layers, and output layer size was kept constant but the size of the first layer and number of input delays were changed. As with the networks tested previously, the Hyperbolic Tangent transfer function was used in the first layer and the Linear function for the output layer. The three structures are represented in Figure 4.18 below. Each network structure was trained three times for 200 epochs.

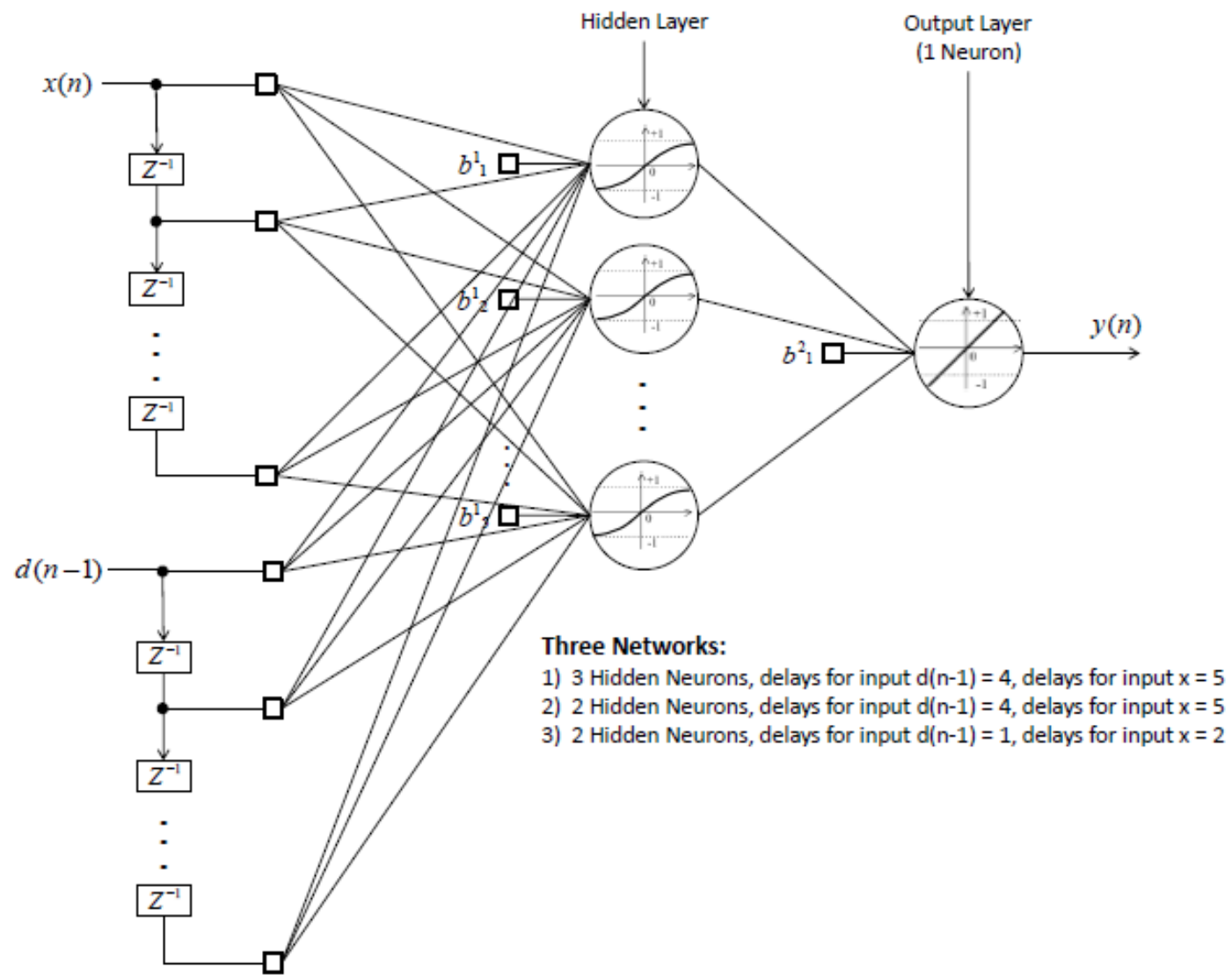

Figure 4.18: NARXSP Networks (3,2 layers with different delays)

The network structures and training results for all NARXSP networks tested thus far are shown in Table 4.1 and Table 4.2 below. 
Table 4.1: NARXSP Structure MSE Comparison

\begin{tabular}{|c|c|c|c|c|c|c|}
\hline $\begin{array}{c}\text { Layer 1 } \\
\text { Size }\end{array}$ & $\begin{array}{c}\text { Input } \\
\text { (VIN) } \\
\text { Delays }\end{array}$ & $\begin{array}{c}\text { Target } \\
\text { Delays }\end{array}$ & $\begin{array}{c}\text { Trial 1 } \\
\text { MSE }\end{array}$ & $\begin{array}{c}\text { Trial 2 } \\
\text { MSE }\end{array}$ & $\begin{array}{c}\text { Trial 3 } \\
\text { MSE }\end{array}$ & $\begin{array}{c}\text { Average } \\
\text { MSE }\end{array}$ \\
\hline 5 & 5 & 5 & $1.3702 \mathrm{E}-7$ & $1.4432 \mathrm{E}-7$ & $1.4169 \mathrm{E}-7$ & $1.4101 \mathrm{E}-7$ \\
\hline 3 & 5 & 5 & $1.4255 \mathrm{E}-7$ & $1.4543 \mathrm{E}-7$ & $1.4353 \mathrm{E}-7$ & $1.4384 \mathrm{E}-7$ \\
\hline 2 & 5 & 5 & $1.4443 \mathrm{E}-7$ & $1.3912 \mathrm{E}-7$ & $1.4309 \mathrm{E}-7$ & $1.4221 \mathrm{E}-7$ \\
\hline 2 & 2 & 2 & $9.1823 \mathrm{E}-7$ & $9.1316 \mathrm{E}-7$ & $8.7917 \mathrm{E}-7$ & $9.0352 \mathrm{E}-7$ \\
\hline
\end{tabular}

Table 4.2: NARXSP Structure $R^{\wedge} 2$ Comparison

\begin{tabular}{|c|c|c|c|c|c|c|}
\hline $\begin{array}{c}\text { Layer 1 } \\
\text { Size }\end{array}$ & $\begin{array}{c}\text { Input } \\
\text { (VIN) } \\
\text { Delays }\end{array}$ & $\begin{array}{c}\text { Target } \\
\text { Delays }\end{array}$ & $\begin{array}{c}\text { Trial 1 } \\
\boldsymbol{R}^{\mathbf{2}}\end{array}$ & $\begin{array}{c}\text { Trial 2 } \\
\boldsymbol{R}^{\mathbf{2}}\end{array}$ & $\begin{array}{c}\text { Trial 3 } \\
\boldsymbol{R}^{\mathbf{2}}\end{array}$ & $\begin{array}{c}\text { Average } \\
\boldsymbol{R}^{\mathbf{2}}\end{array}$ \\
\hline 5 & 5 & 5 & 0.99999 & 0.99999 & 0.99999 & 0.99999 \\
\hline 3 & 5 & 5 & 0.99999 & 0.99999 & 0.99999 & 0.99999 \\
\hline 2 & 5 & 5 & 0.99999 & 0.99999 & 0.99999 & 0.99999 \\
\hline 2 & 2 & 2 & 0.99994 & 0.99994 & 0.99994 & 0.99994 \\
\hline
\end{tabular}

It can be seen from the above tables that the NARXSP network decreases in performance (MSE) only slightly with a decrease in layer size and number of delays. However, reducing the number of delays seems to affect (worsen) the results more so than decreasing the number of neurons. Table 4.3: Network Structure Comparison below summarizes the performances of the seven networks tested.

Table 4.3: Network Structure Comparison

\begin{tabular}{|c|c|c|}
\hline Network & $\begin{array}{l}\text { Average } \\
\text { MSE }\end{array}$ & $\begin{array}{l}\text { Average } \\
R^{2}\end{array}$ \\
\hline $\mathrm{FF}$ & 0.014151 & 0.0028621 \\
\hline FTDNN & 0.005924 & 0.58258 \\
\hline NARX & 0.0067479 & 0.52972 \\
\hline $\begin{array}{l}\text { NARXSP ( } 5 \text { hidden neurons, } 5 \text { delay TDL on input } \mathrm{x}, 4 \\
\text { delay TDL on input } \mathrm{d}(\mathrm{n}-1) \text { ) }\end{array}$ & $1.4101 \mathrm{E}-7$ & 0.99999 \\
\hline $\begin{array}{l}\text { NARXSP (3 hidden neurons, } 5 \text { delay TDL on input } \mathrm{x}, 4 \\
\text { delay TDL on input } \mathrm{d}(\mathrm{n}-1) \text { ) }\end{array}$ & $1.4384 \mathrm{E}-7$ & 0.99999 \\
\hline $\begin{array}{l}\text { NARXSP (2 hidden neurons, } 5 \text { delay TDL on input } \mathrm{x}, 4 \\
\text { delay TDL on input } \mathrm{d}(\mathrm{n}-1))\end{array}$ & $1.4221 \mathrm{E}-7$ & 0.99999 \\
\hline $\begin{array}{l}\text { NARXSP ( } 2 \text { hidden neurons, } 2 \text { delay TDL on input } x, 1 \\
\text { delay TDL on input } \mathrm{d}(\mathrm{n}-1))\end{array}$ & $9.0352 \mathrm{E}-7$ & 0.99994 \\
\hline
\end{tabular}


The results in the table above indicate that the NARXSP network structure yielded the lowest MSE, and the best R-Squared value (closest to one) from the structures tested. Given the $5 \mathrm{~Hz}$ sine wave training set this network structure was able to model the system very accurately with an average R-Squared value of 0.99999 . The standard Feedforward network gave the worst results with an R-squared value of 0.0028621 . The fact that the NARXSP network was able to model the data more accurately coincides with the observation that the data is time-variant. This can be seen from looking at the plot of the input/target training set (Figure 3.2). It was also shown that reducing the number of neurons and delays for the NARXSP type structure did not have a significant impact on the mean squared error.

\section{3 - Training and Simulation Results for Each Data Set}

The modeling of the neural network was next studied for every input/target data set experimentally measured. The network however was not trained to the $5 \mathrm{~Hz}$ sine wave training set since the same network structure (mentioned below) was already trained to this signal. The Serial-Parallel Nonlinear Autoregressive Network with Exogenous Inputs (NARXSP) was chosen as the network structure. This structure was chosen because it performed well when training to the $5 \mathrm{~Hz}$ sine wave in section 5.2. Also, the smallest NARXSP structure size was chosen ( 2 neurons in hidden layer with 2 delays) because there was little difference in MSE or R-squared values between the smallest and largest network for this type of structure, and because a smaller network will train faster. The 
network is shown in Figure 4.19 below. The network was trained three times for each input/target data set and the results are shown in the figures below.

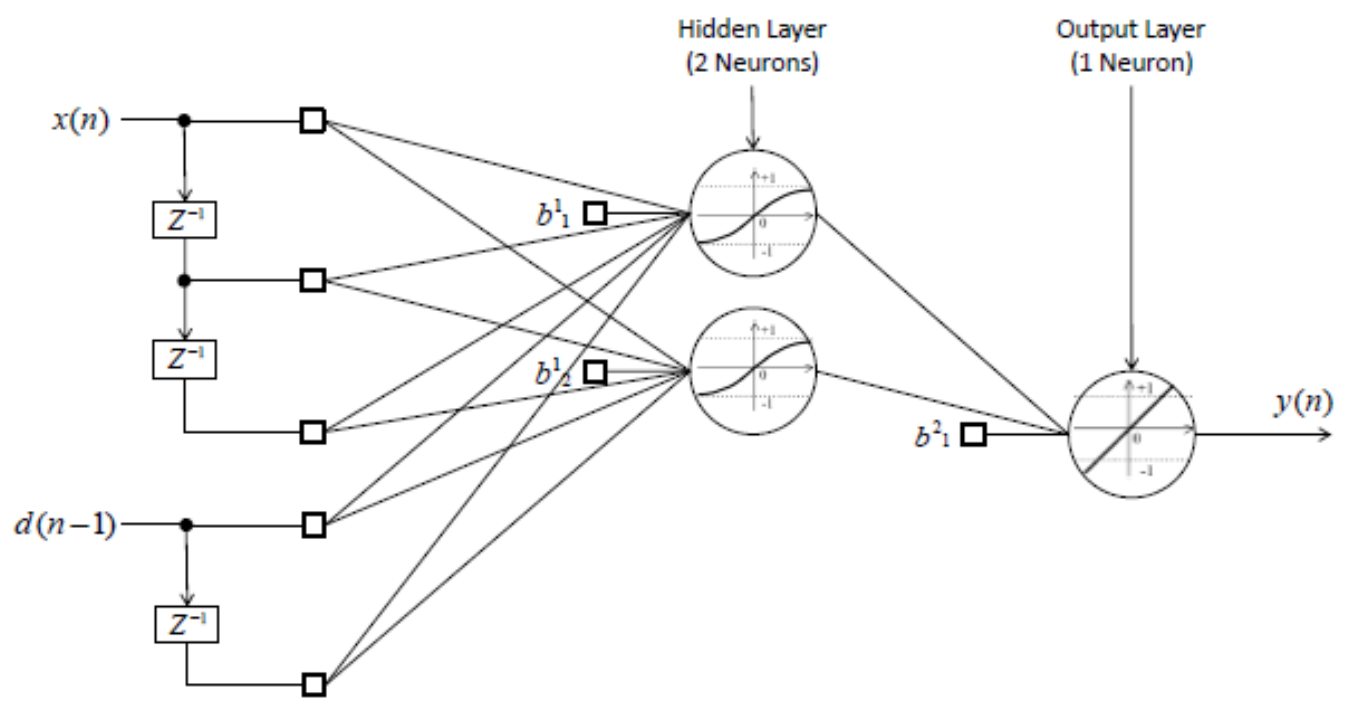

Figure 4.19: 2 Neuron NARXSP Network

The network was first trained to the Chirp signal (Figure 3.13). After 200 epochs the MSE values for the three trials were 1.0182E-6, 1.0194E-6, and 1.0177E-6. The Rsquared values were as follows: $0.99576,0.99576,0.99577$. The plot of MSE vs. training epoch and simulation results of the last trial are shown in Figure 4.20 through Figure 4.23 below. 


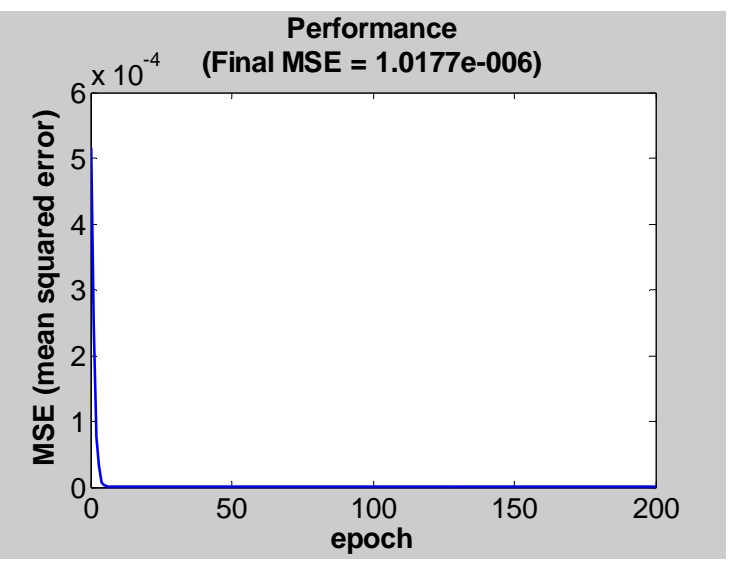

Figure 4.20: Performance (Chirp Signal)

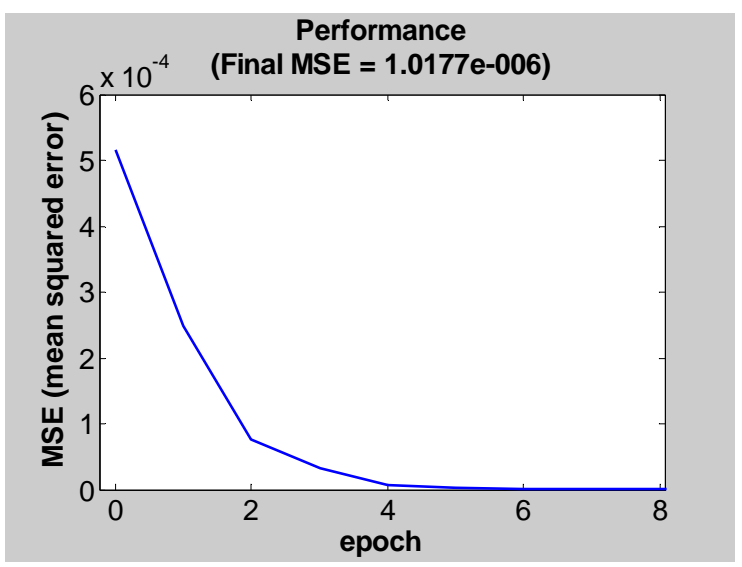

Figure 4.21: Performance (Chirp Signal) Zoomed

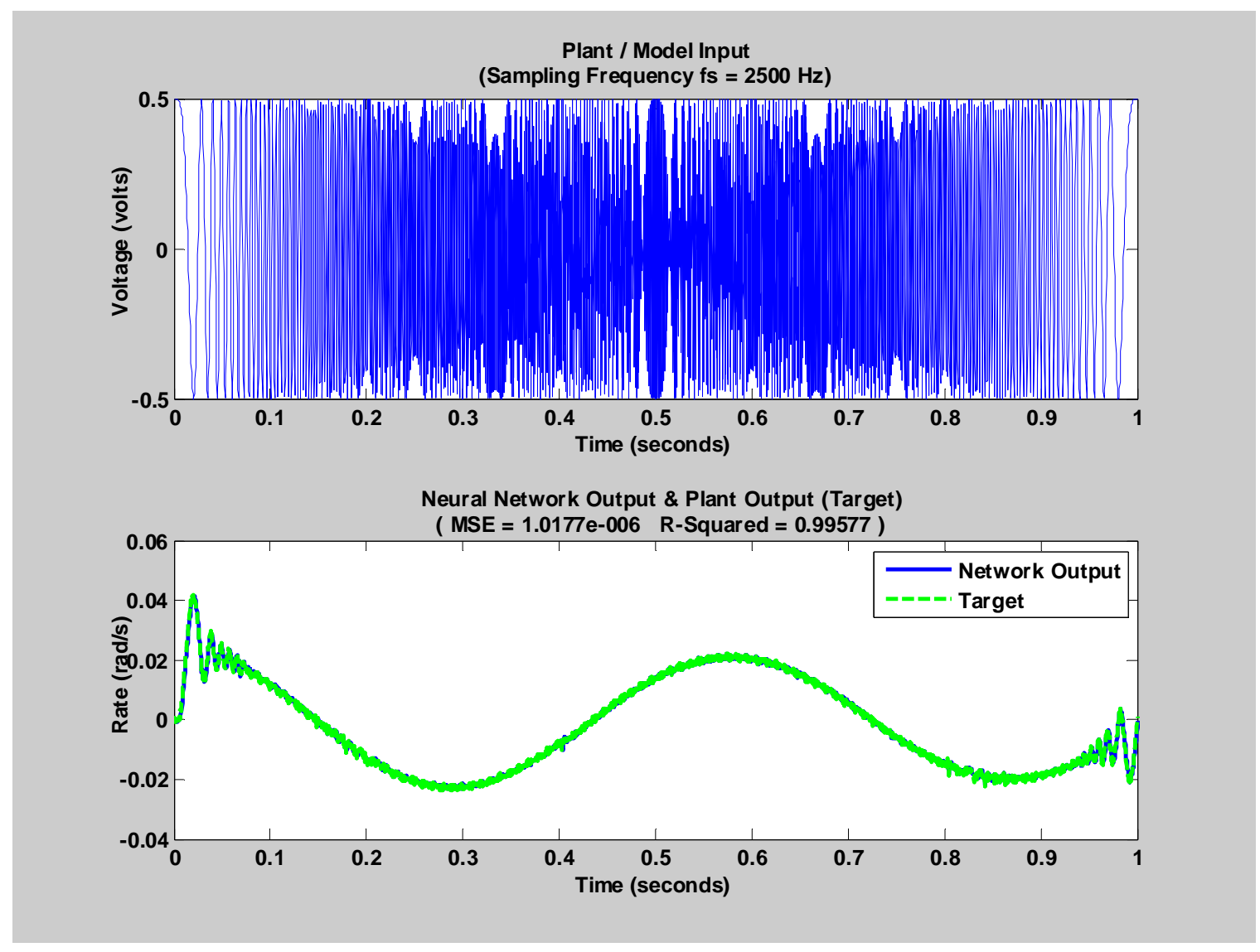

Figure 4.22: Simulation: Chirp Signal 

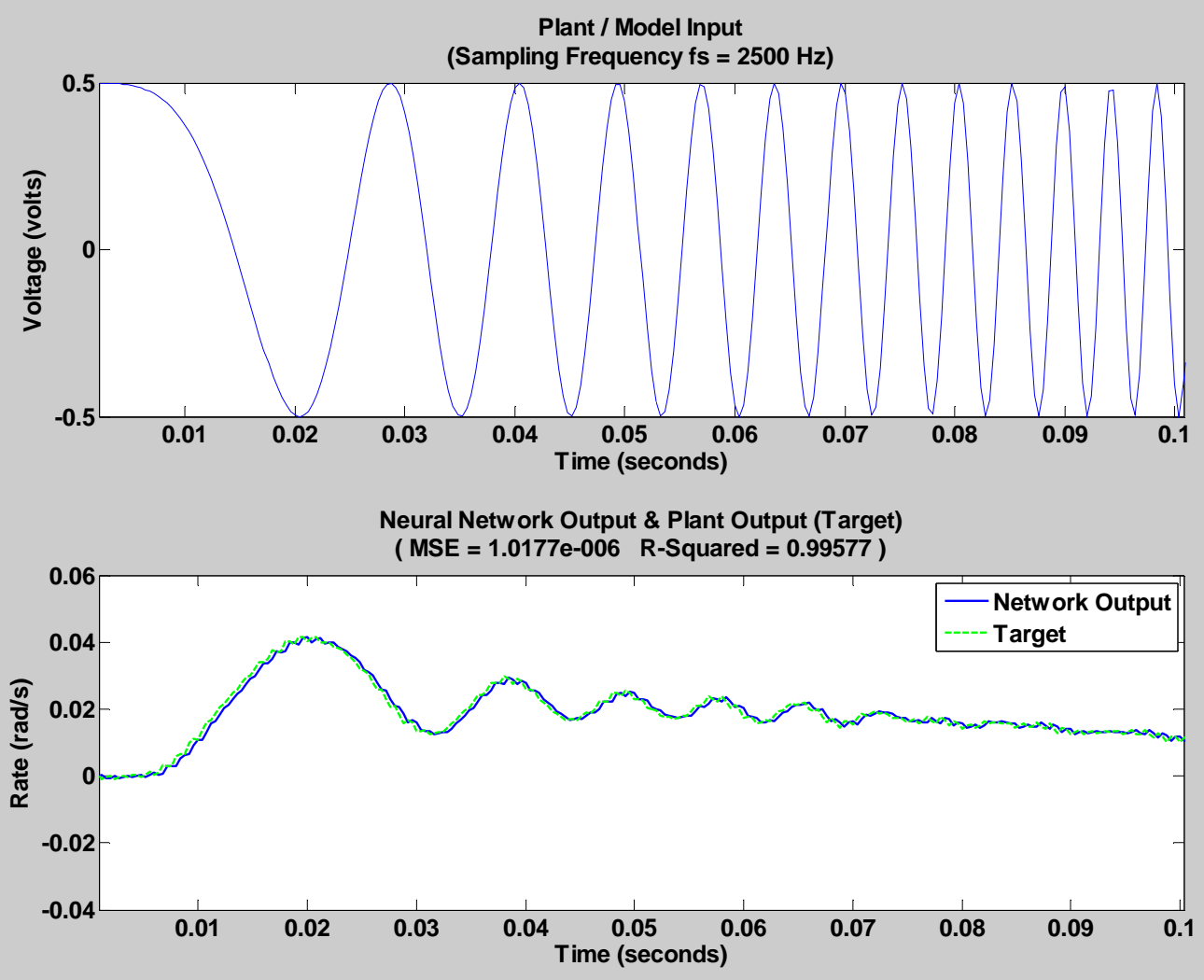

Figure 4.23: Simulation: Chirp Signal Zoomed (1st 0.1 seconds)

The network was next trained to the step function training set (Figure 3.19). After 200 epochs the MSE values for the three trials were 1.1698E-6, 1.1235E-6, and 1.1238E6. The R-squared values for all three trials were 0.99991. The plot of MSE vs. training epoch and the simulation results for the third trial are shown in Figure 4.24 through Figure 4.26 below. 


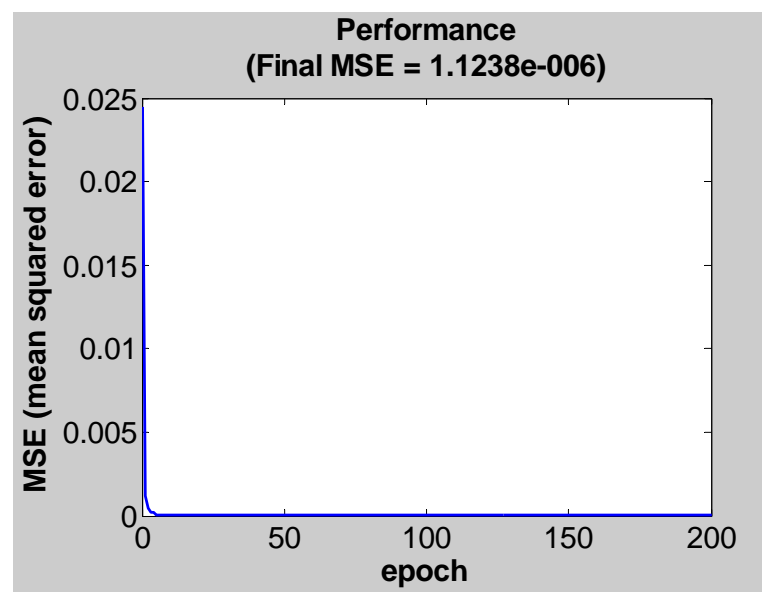

Figure 4.24: Performance (Step Function)

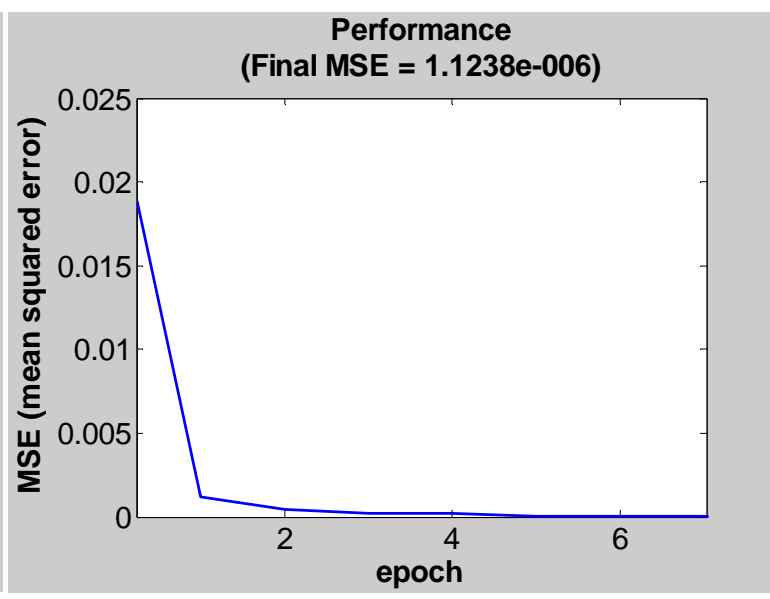

Figure 4.25: performance (Step Function) Zoomed
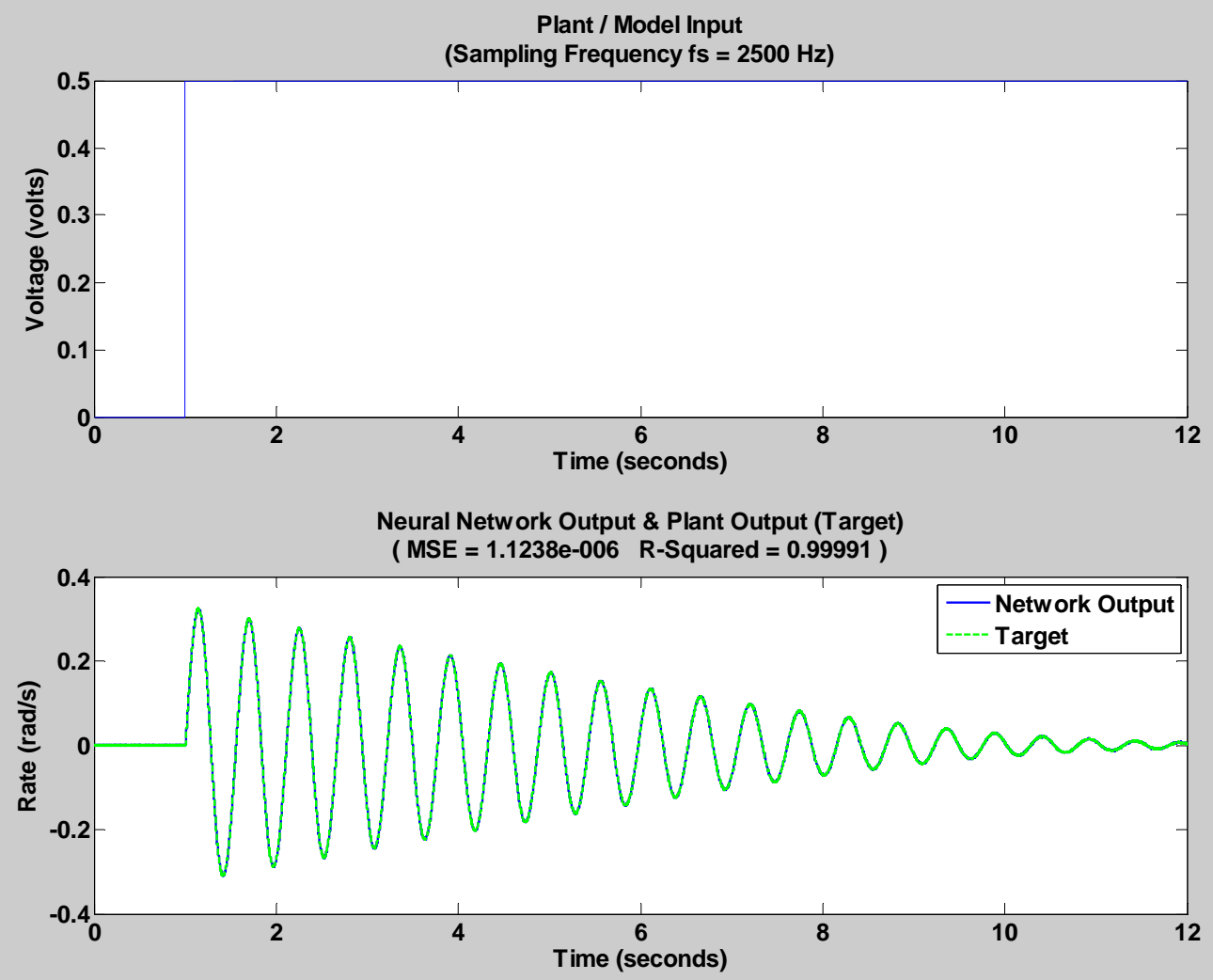

Figure 4.26: Simulation: Step Input (Section 4.3.9)

The network was trained to the random signal data set (Figure 3.18). After 200 epochs the MSE values for the three trials were each 2.0339-6, 1.9789E-6, 2.0172E-6. 
The R-squared values for all three trials as 0.99997 . The training and simulation results for the third and last trial are shown in Figure 4.27 through Figure 4.29 below.

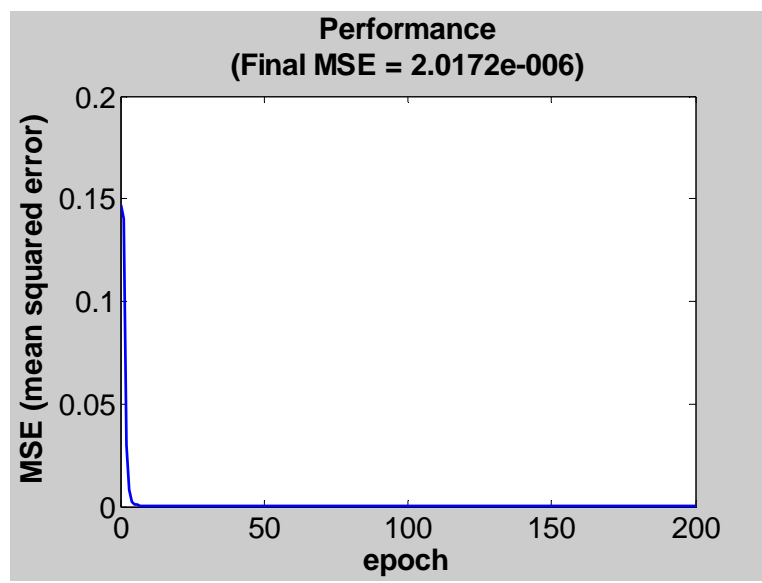

Figure 4.27: Performance (Random Signal)

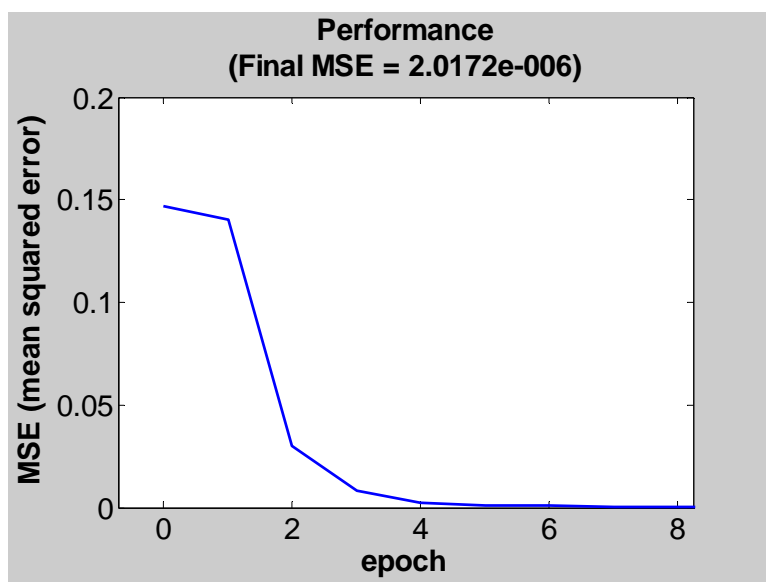

Figure 4.28: Performance (Random Signal) Zoomed
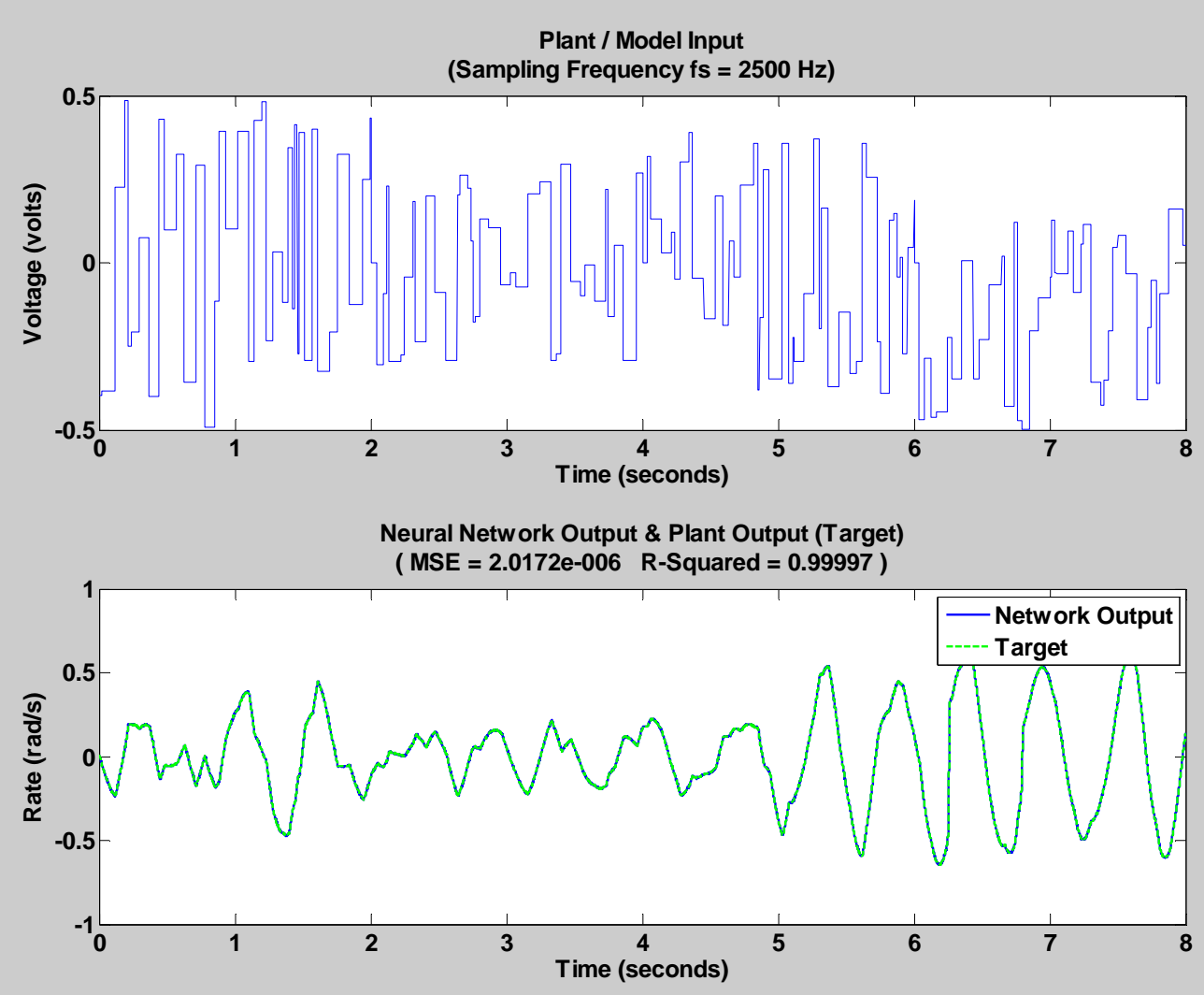

Figure 4.29: Simulation: Random Signal (Section 4.3.8) 
The network was next trained and simulated using the multiple frequency signal data set (Figure 3.6). After 200 epochs the MSE values for the three trials were 1.8523E6, 1.859E-6, and 1.8167E-6. The R-squared values for all three trials were 0.99994. The training and simulation results for the last trial are shown in Figure 4.30 through Figure 4.32 below.

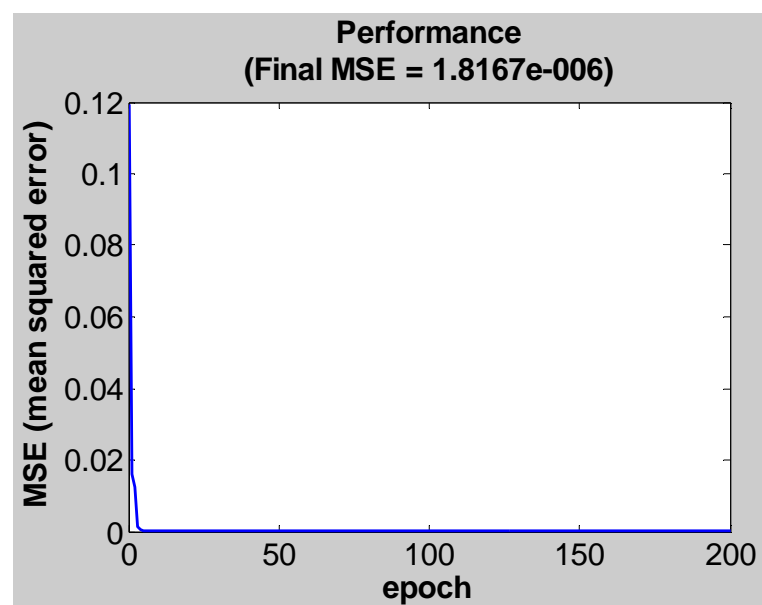

Figure 4.30: Performance (Mult Freq)

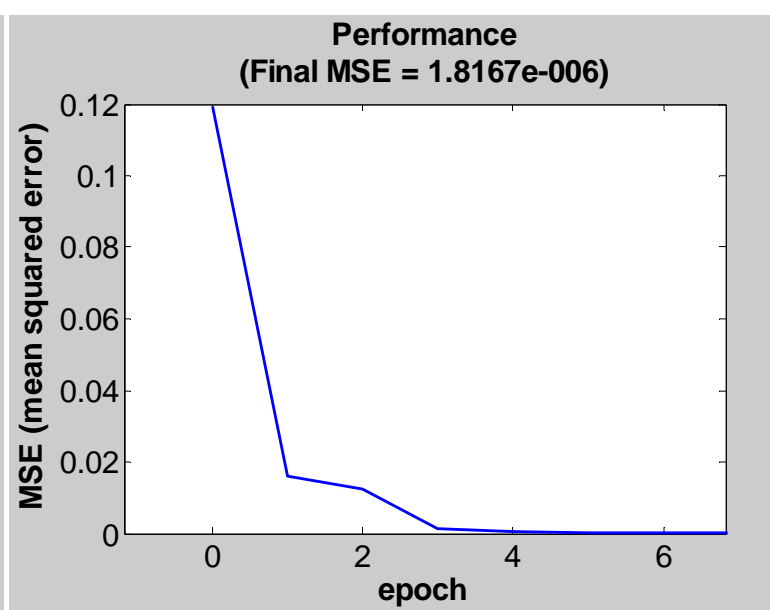

Figure 4.31: Performance (Mult Freq) Zoomed 

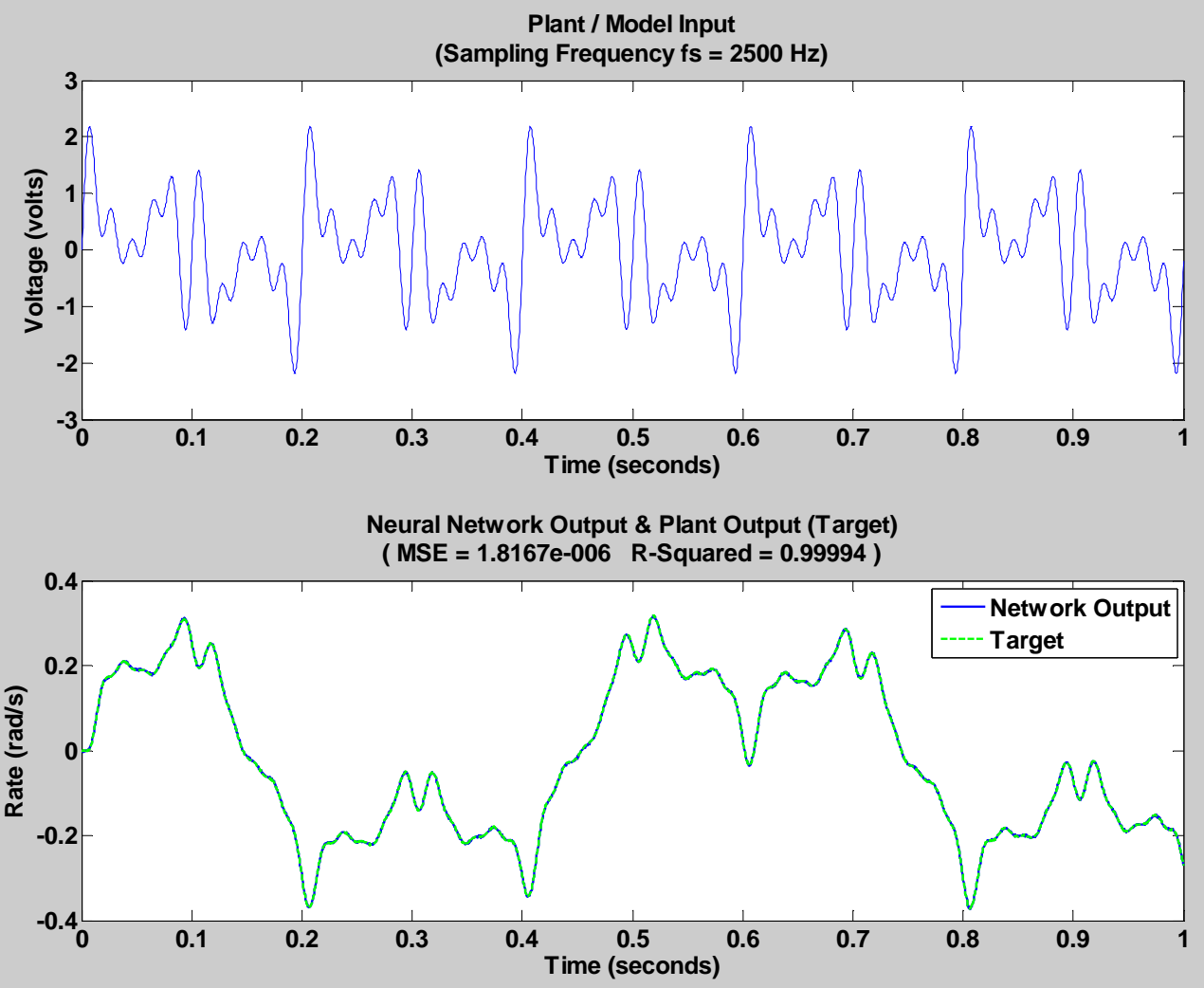

Figure 4.32: Simulation: Multiple Frequency Wave (Section 4.3.4)

The network was then trained to the multiple sine wave and multiple frequency wave appended (Figure 3.7). After 200 epochs the MSE values for the three trials were each 1.4655E-6, 1.4535E-6, 1.4565E-6, The R-squared value for all three trials was 0.99972. The results of trial 3 of the training and simulation are shown in Figure 4.33 through Figure 4.36 below. 


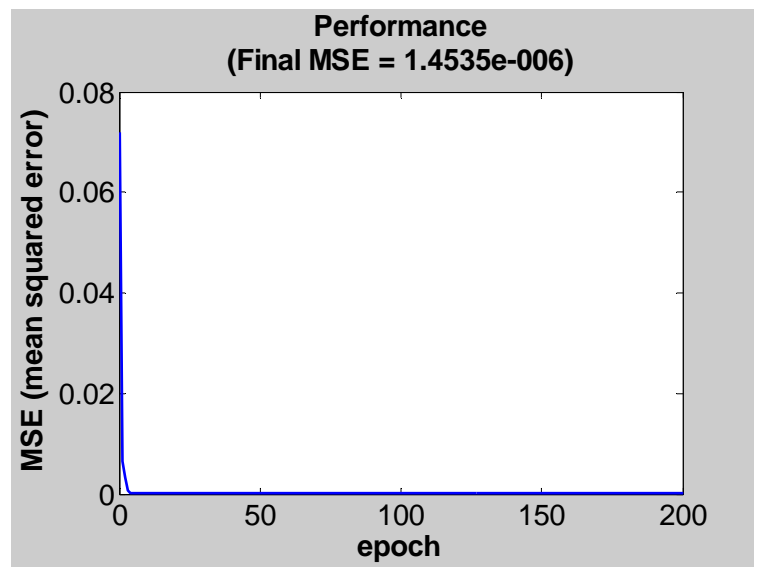

Figure 4.33: Performance (Mult Single Freq w/ Mult)

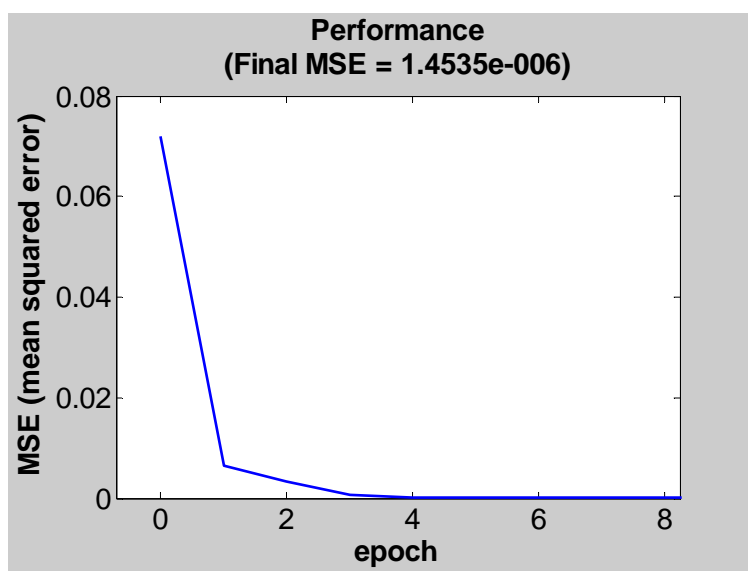

Figure 4.34: Performance (Mult Single Freq w/ Mult) Zoomed

Plant I Model Input

(Sampling Frequency $\mathrm{fs}=2500 \mathrm{~Hz}$ )

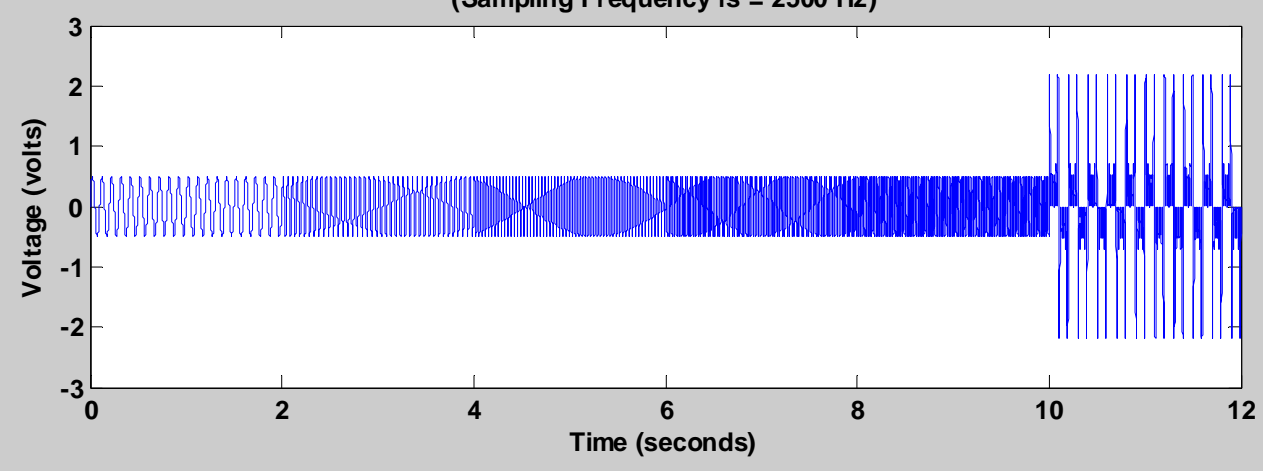

Neural Network Output \& Plant Output (Target)

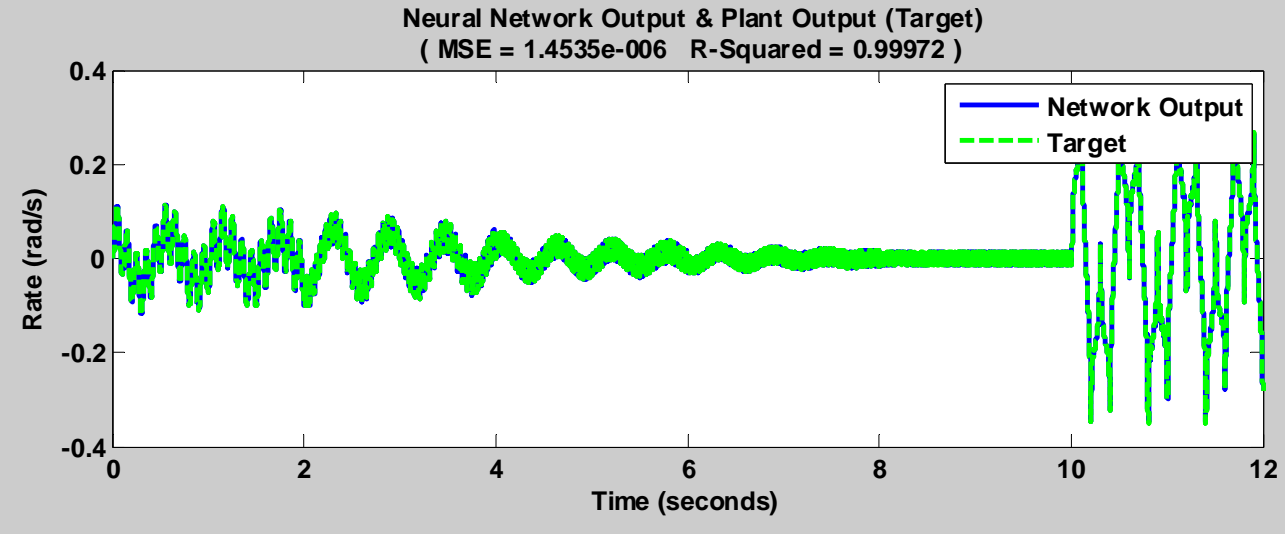

Figure 4.35: Simulation: Single \& Multiple Frequency Waves 


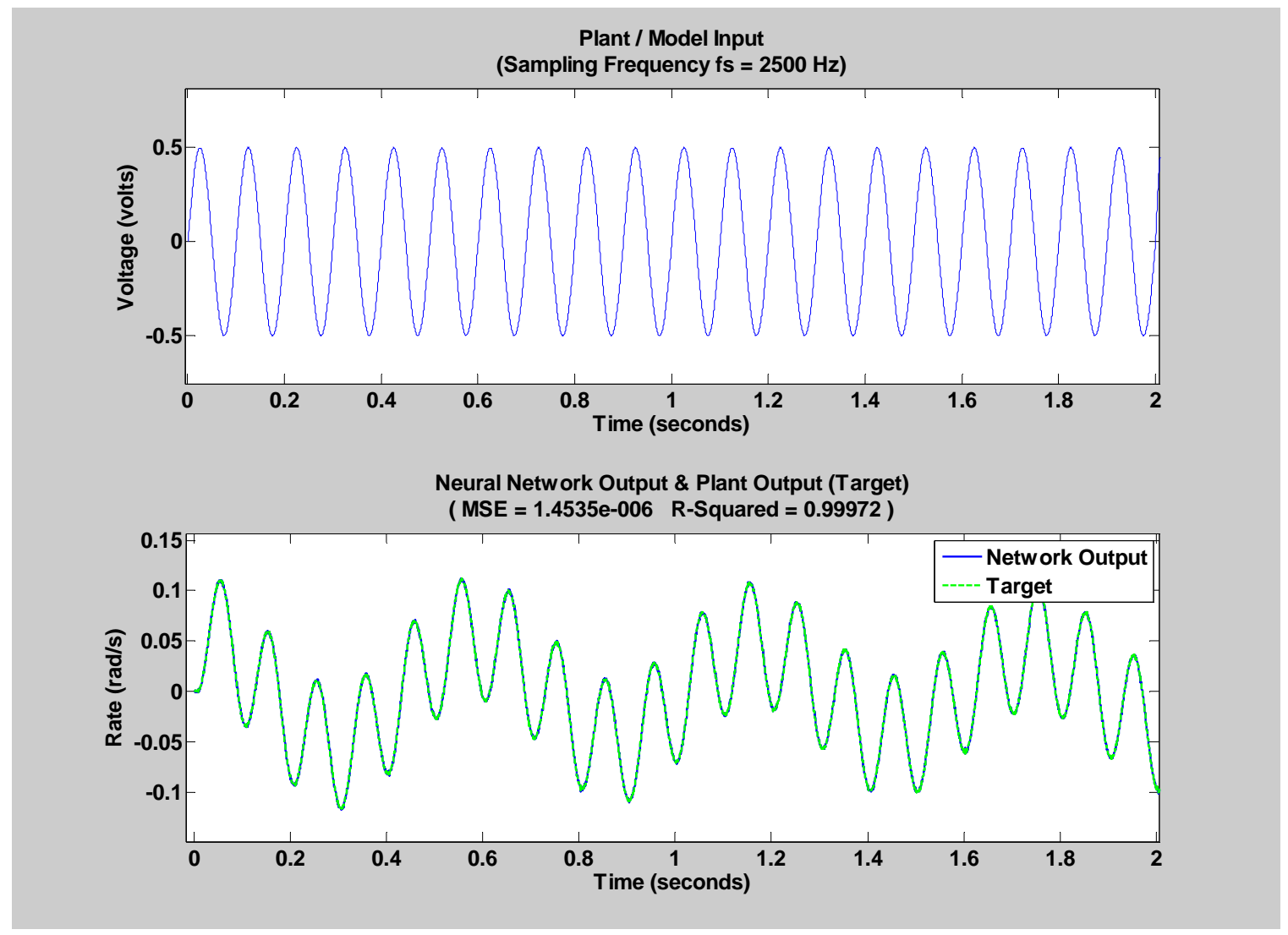

Figure 4.36: Simulation: Single \& Multiple Frequency Waves Zoomed

The last set of input/target pairs was the $35 \mathrm{~Hz}$ sine wave input (Figure 3.4). After 200 epochs the MSE values for the three trials were 7.916E-7, 7.8916E-7, and 7.9052E-7. The R-squared values were $0.99571,0.99573$, and, 0.99572. The simulation results are show in Figure 4.37 through Figure 4.40 below. 


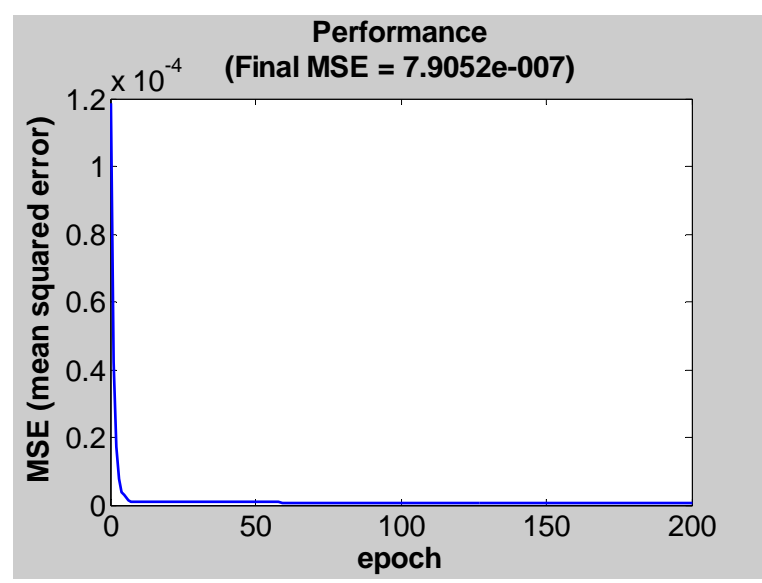

Figure 4.37: Performance ( $35 \mathrm{~Hz}$ Sine Wave)

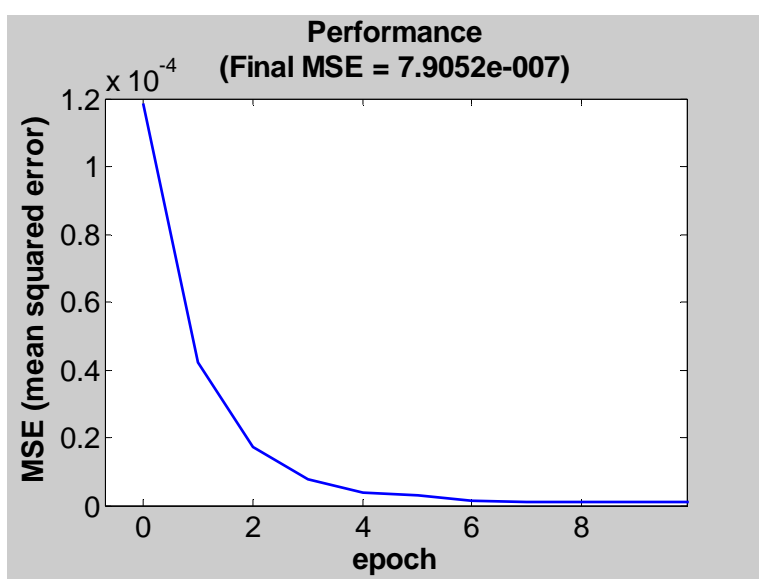

Figure 4.38: Performance ( $35 \mathrm{~Hz}$ Sine Wave) Zoomed
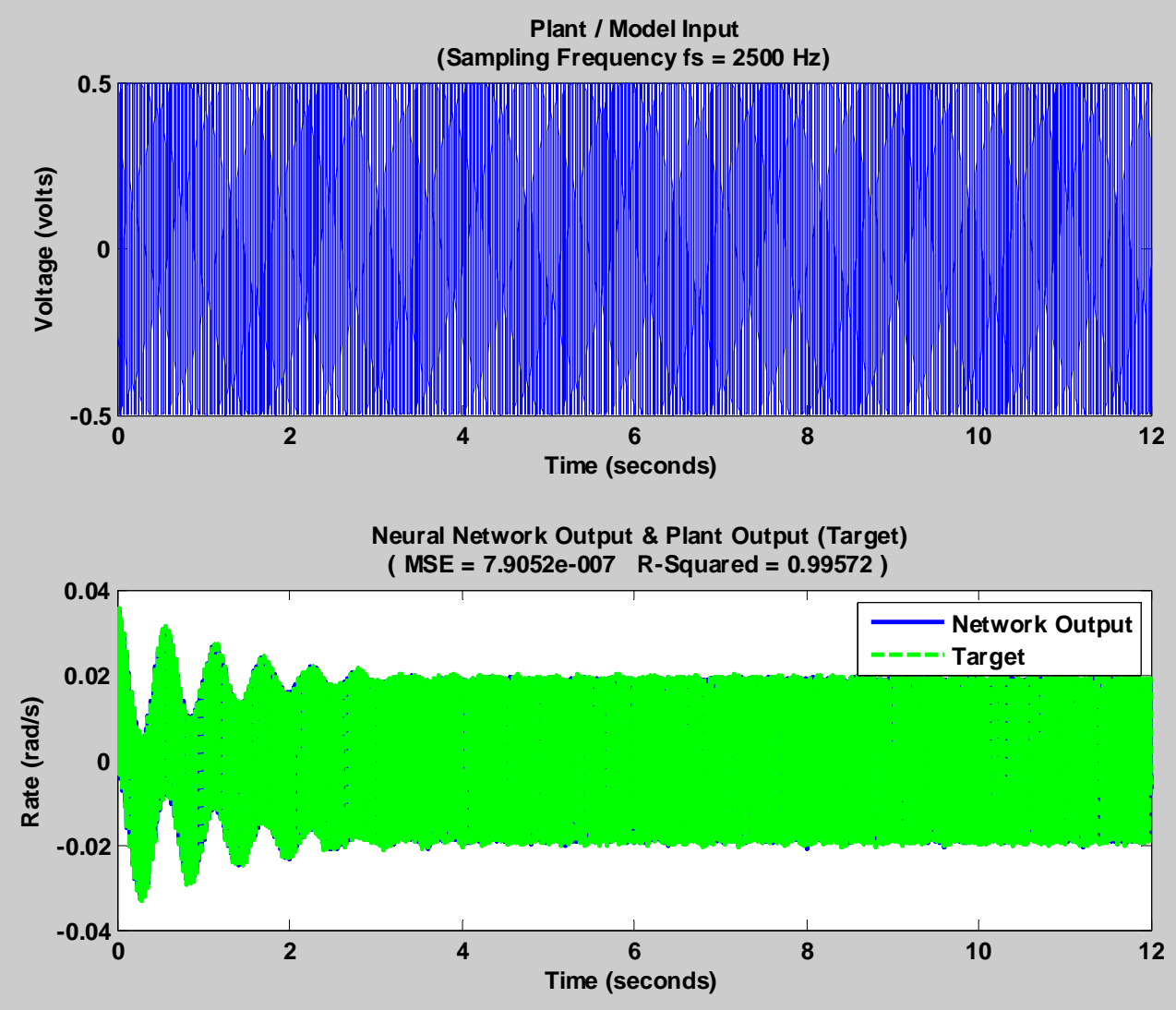

Figure 4.39: Simulation: $35 \mathrm{~Hz}$ Sine Wave 


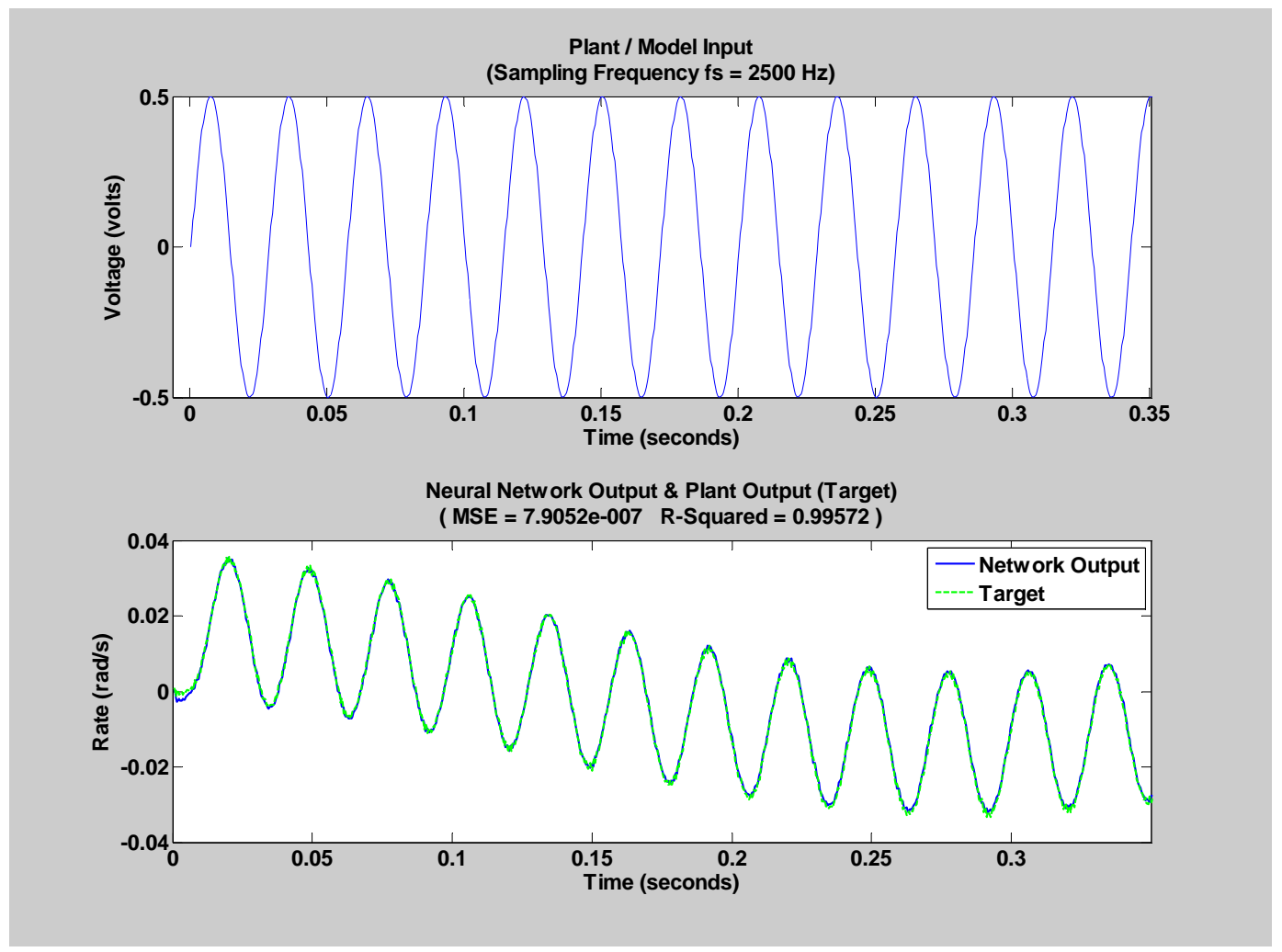

Figure 4.40: Simulation: $35 \mathrm{~Hz}$ Sine Wave Zoomed ( $1^{\text {st }} 0.35$ seconds)

The neural network was able to model the inner azimuth gimbal accurately for the 7 input/target data sets measured. A summary of the average mean squared error and Rsquared values is shown in Table 4.4 below. Note that the results for the $5 \mathrm{~Hz}$ sine wave input were obtained in the previous section 4.3. The simulation result figures and the table below show that the neural network was able to accurately model the system for all input types tested. This is indicated by the similarities between plots and the low mean squared error values. In addition, the R-squared values are very close to 1, indicating that the network model output accurately models the real system output. It is interesting to note that the random signal has the second worst average MSE value but had the best Rsquared value. The network had the most difficulty training to the $35 \mathrm{~Hz}$ sine wave with 
an average MSE of 7.9007E-6 (the largest MSE of training sets). The $5 \mathrm{~Hz}$ sine wave had the lowest MSE value (9.0352E-7).

Table 4.4: Plant Modeling for Several Data Sets

\begin{tabular}{|c|c|c|}
\hline Input/Target Data Type & Average MSE & Average R-Squared \\
\hline Chirp Signal & $1.0184 \mathrm{E}-6$ & 0.99576 \\
\hline Step Signal & $1.1390 \mathrm{E}-6$ & 0.99991 \\
\hline Random Signal & $2.0100 \mathrm{E}-6$ & 0.99997 \\
\hline Multiple Frequency Signal & $1.8427 \mathrm{E}-6$ & 0.99994 \\
\hline Multiple \& Single Frequency & $1.4585 \mathrm{E}-6$ & 0.99972 \\
\hline 5 Hz Sine Wave & $9.0352 \mathrm{E}-7$ & 0.99994 \\
\hline 35 Hz Sine Wave & $7.9007 \mathrm{E}-6$ & 0.99572 \\
\hline
\end{tabular}

\section{4 - Network Generalization Study}

It has been shown that the neural network is able to model the plant (inner azimuth gimbal) accurately for all the experimentally measured input/target data sets. However, it is important to also study how well the neural network can generalize over the full operating range of the system.

In order to test for generalization the network is trained to one training set. The weights are then held constant while the network output is simulated for each input signal mentioned in Chapter 3. The MSE and R-squared values are then calculated by comparing the neural network output with the real system output for each training set. If the network generalizes well, the MSE values should be small, and the R-squared value should be close to 1 for all training sets.

The input/target set chosen to train the network was the random signal data set (Figure 3.18). Again, the equation for this signal is: 


$$
x(t)=\left\{\begin{array}{lr}
a_{1}+\left(b_{1}-a_{1}\right) \cdot \operatorname{rand}(1), & 0 \leq t<t_{1} \\
a_{m}+\left(b_{m}-a_{m}\right) \cdot \operatorname{rand}(1), & t_{m-1} \leq t<t_{m} \\
a_{p}+\left(b_{p}-a_{p}\right) \cdot \operatorname{rand}(1), & t_{p-1} \leq t \leq t_{p}
\end{array}\right.
$$

Where

$$
t_{m}=t_{m-1}+.01+(.1-.01) \cdot \operatorname{rand}(1)
$$

And

$$
a_{m}=\left\{\begin{array}{cc}
-0.5, & 0 \leq t<2 \\
-0.33, & 2 \leq t<4 \\
-0.4, & 4 \leq t<6 \\
-0.5, & 6 \leq t \leq 8
\end{array}\right.
$$

And

$$
b_{m}=\left\{\begin{array}{cc}
0.5, & 0 \leq t<2 \\
0.33, & 2 \leq t<4 \\
0.4, & 4 \leq t<6 \\
.167, & 6 \leq t \leq 8
\end{array}\right.
$$

The sampling frequency was $f_{s}=2500 \mathrm{~Hz}$ and the total time duration was $t_{p}=8$ seconds. This signal is chosen because it contains frequencies up to the Nyquist frequency, is pseudorandom, and contains a large amount of energy in the frequency range spanning the servo bandwidth of the system (below $45 \mathrm{~Hz}$ ). The frequency spectrum for this signal is shown in Figure 4.41 below. Note that the spectrum is not flat because the signal is not completely random. 


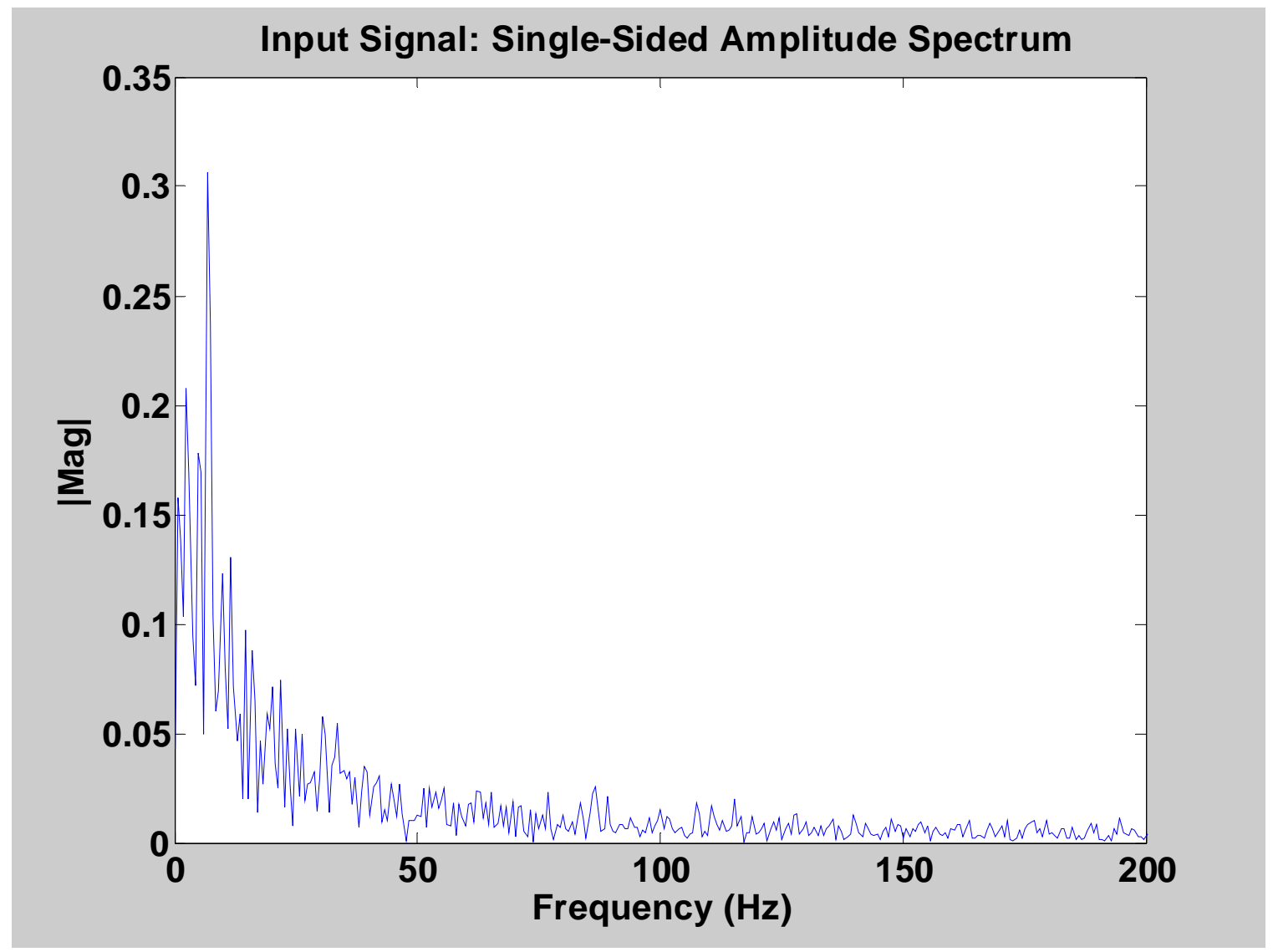

Figure 4.41: Frequency Spectrum for the Random Input Signal

The NARXSP neural network structure used in Section 4.3 in Figure 4.19 is again used for this study and was trained one time for 200 epochs. To help define the weights of the network, the structure is shown in Figure 4.42 below with the weights labeled. 


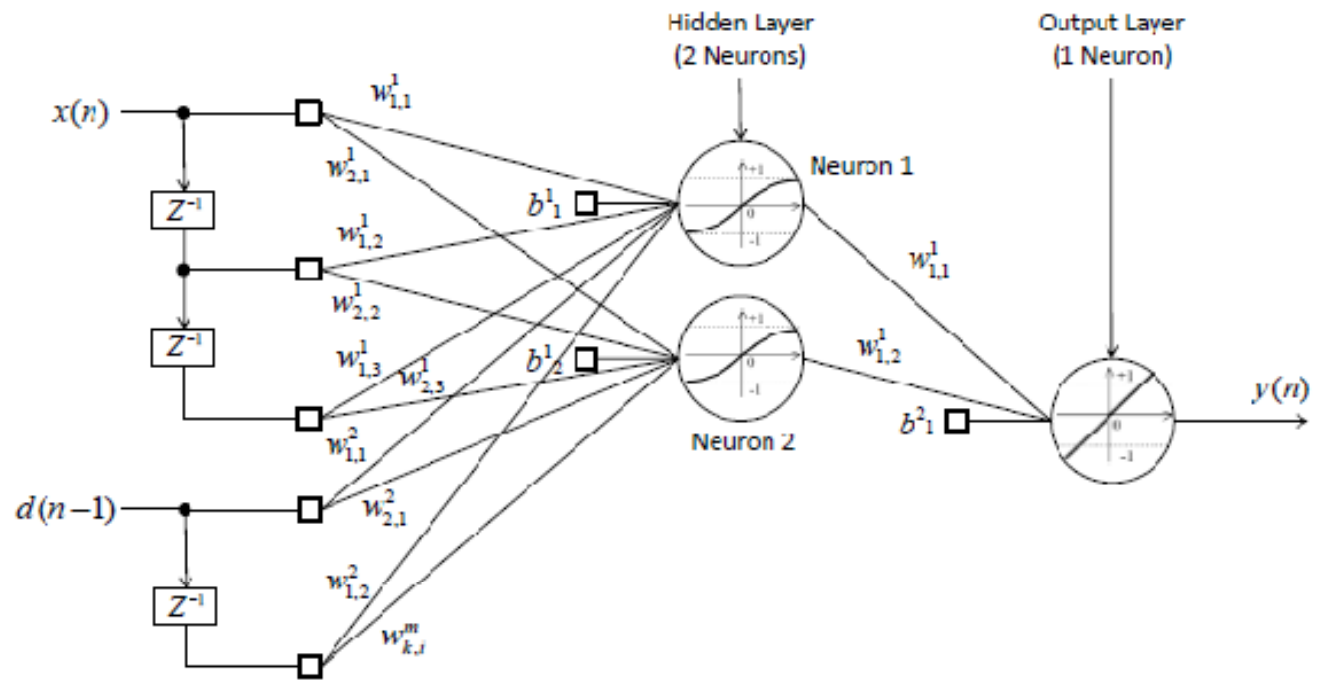

Figure 4.42: Generalization Study: NARXSP Network

In reference to the above figure: the input weights are of the form $w_{k, j}^{m}$ where $\mathrm{m}$ defines the input vector with $\mathrm{m}=1$ for system input vector $\mathrm{X}$ and $\mathrm{m}=2$ for target vector D. The value $k$ is the neuron in the first layer and $\mathrm{j}$ is the element of vector $\mathrm{m}$. The weight vector for layer 1 neuron 1 is $W_{1}^{1}=\left[w_{1,1}^{1} w_{1,2}^{1} w_{1,3}^{1} w_{1,1}^{2} w_{1,2}^{2}\right]=\left[\begin{array}{lll}0.0000 & 0.0001-\end{array}\right.$ $.0003-.3276 .1039]$ and the weight vector for layer 1 neuron 2 is $W_{2}^{1}=$ $\left[w_{2,1}^{1} w_{2,2}^{1} w_{2,3}^{1} w_{2,1}^{2} w_{2,2}^{2}\right]=\left[\begin{array}{lllll}-0.0003 & 0.0014 & -.0010 & 0.8781 & -.4762\end{array}\right]$. Note that the input weight vector can be defined as $W^{1}=\left[W_{1}^{1} W_{2}^{1}\right]$. The weight vector between the hidden layer and the output layer is $W^{2}=\left[w_{1,1}^{1}, w_{1,2}^{1}\right]=[-4.07361 .2295]$. Lastly, the bias's are $b_{1}^{1}=0.4443, b_{2}^{1}=0.8810$, and $b_{1}^{2}=0.8302$. After training the network the mean squared error MSE and R-squared values were 1.9801E-6, and 0.99997 respectively. The training and simulation results for the Random signal are shown in Figure 4.43 through Figure 4.45 below. 


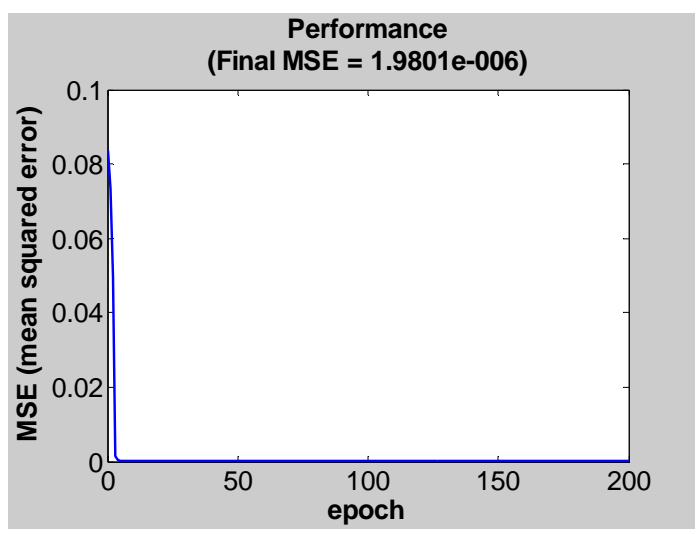

Figure 4.43: Generalization Study - Performance (Random Signal)

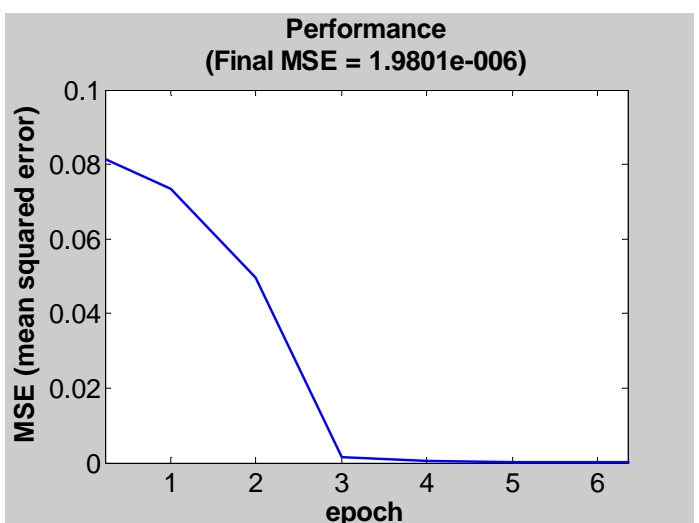

Figure 4.44: Generalization Study - Performance (Random Signal) Zoomed
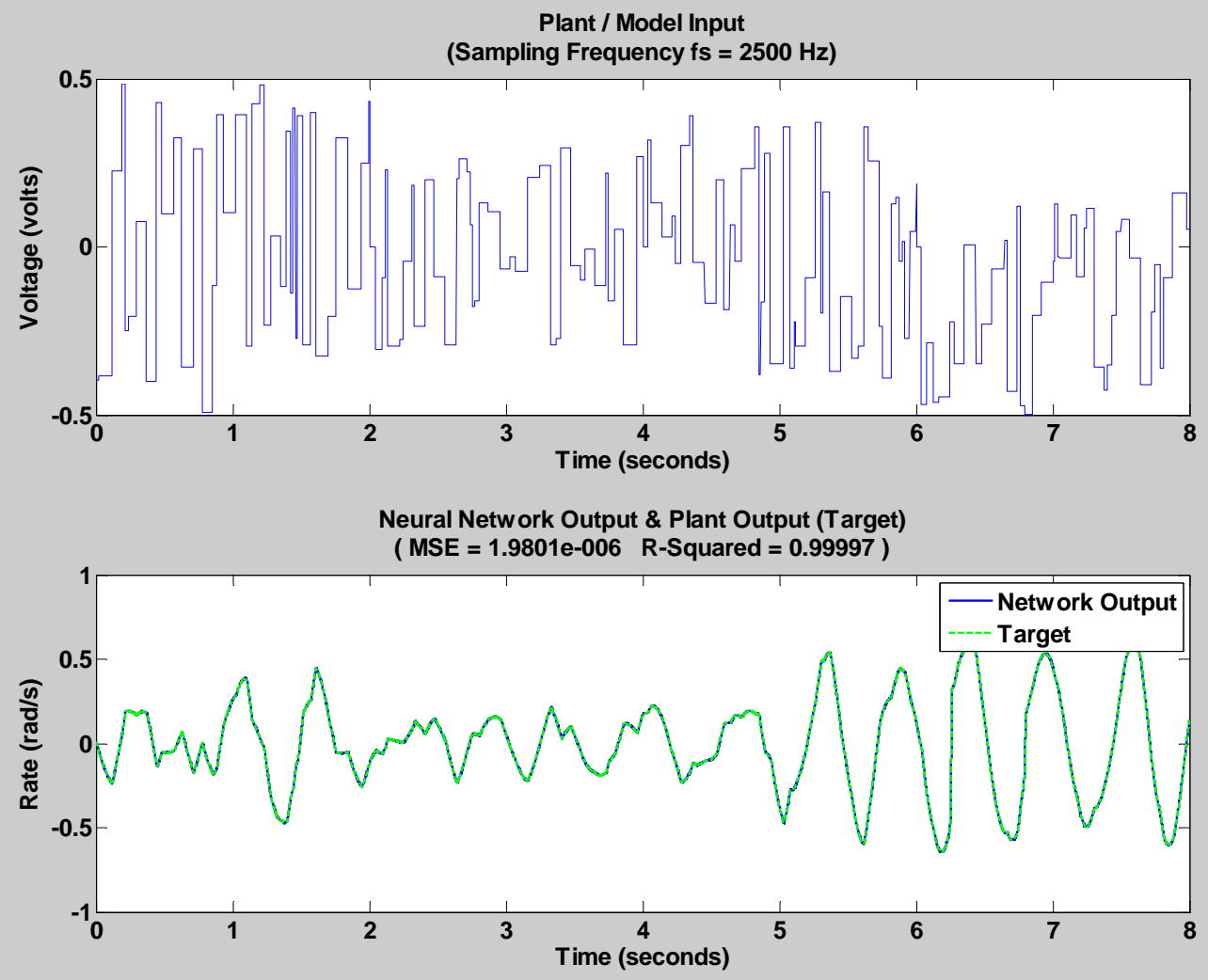

Figure 4.45: Generalization Study Simulation Results (Random Signal) 
Without changing weight values the network response is simulated for each of the remaining six input/target data sets mentioned in Chapter 3. The mean squared error, and R-Squared values are calculated for each data set. The simulation results are shown in the figures below. A summary of the results of this study is given at the end of this section.
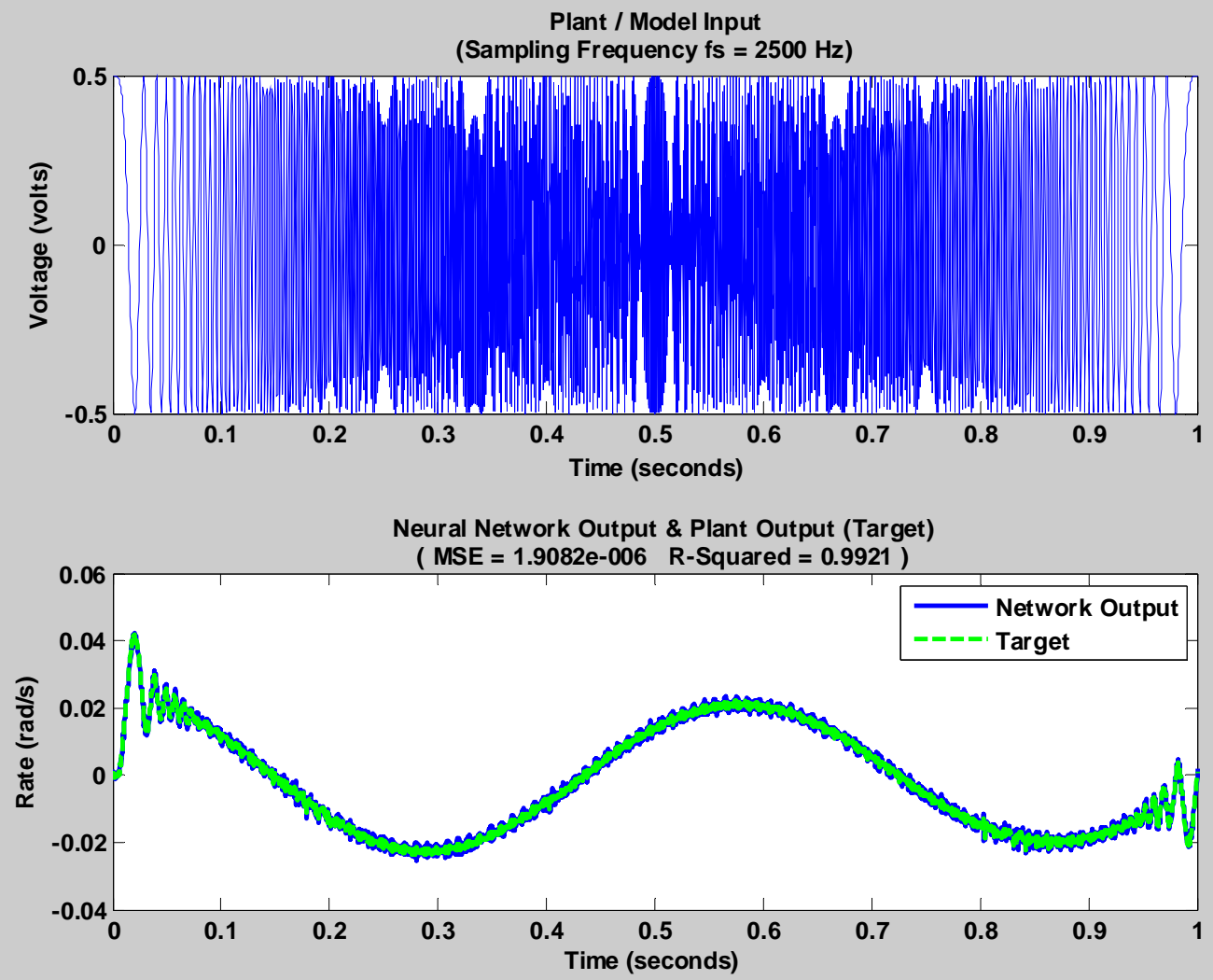

Figure 4.46: Simulation: Chirp Signal (Generalized Network) 


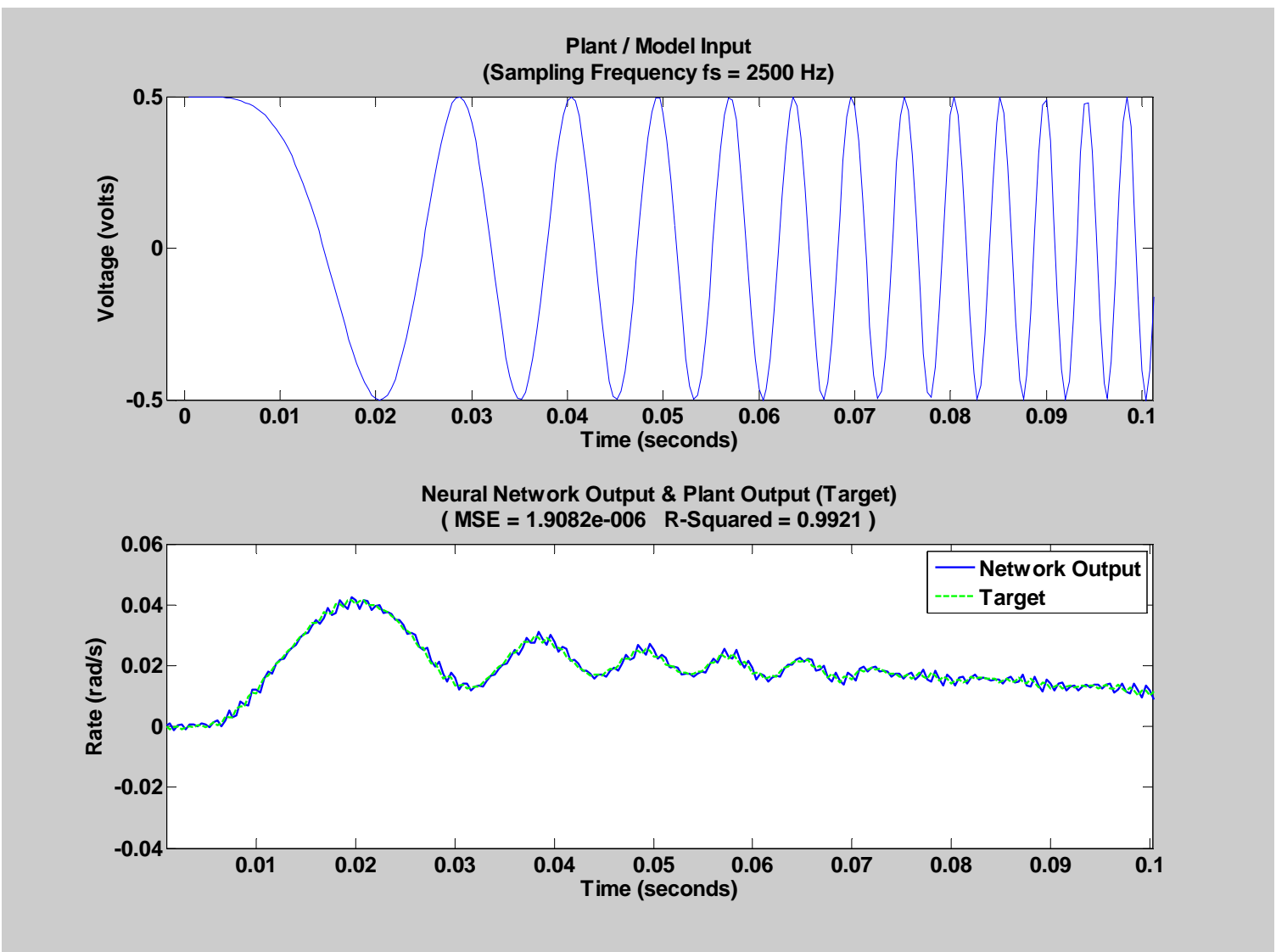

Figure 4.47: Simulation: Chirp Signal (Generalized Network) Zoomed

The first input/target data set used the Chirp signal (Figure 3.13) as input for simulation. The simulations results are shown in Figure 4.46 and Figure 4.47 above. The mean squared error was $1.9082 \mathrm{E}-6$ and the R-squared value was 0.9921 . 
Plant I Model Input

(Sampling Frequency fs $=2500 \mathrm{~Hz}$ )

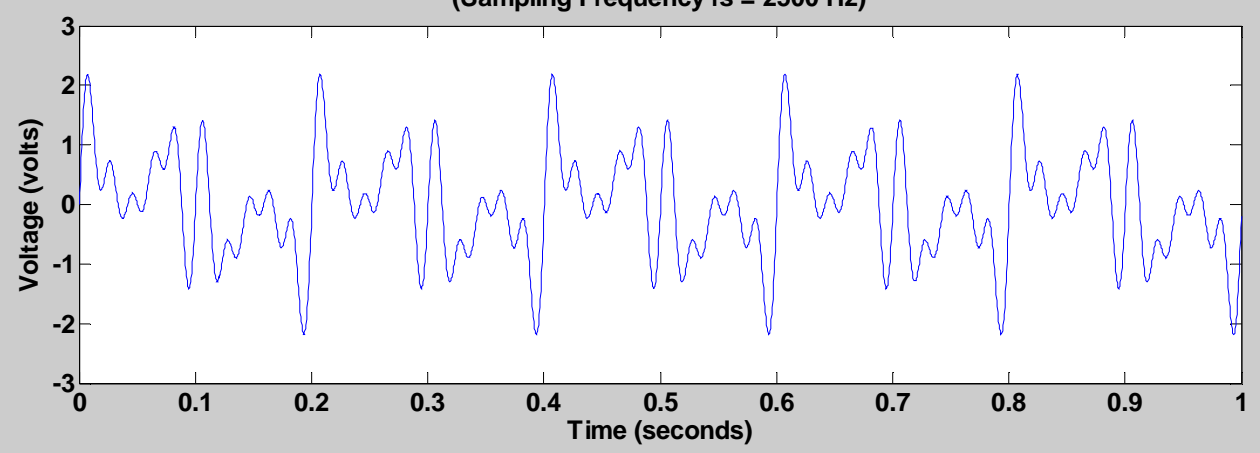

Neural Network Output \& Plant Output (Target)

( MSE $=2.2514 \mathrm{e}-006$ R-Squared $=0.99993$ )

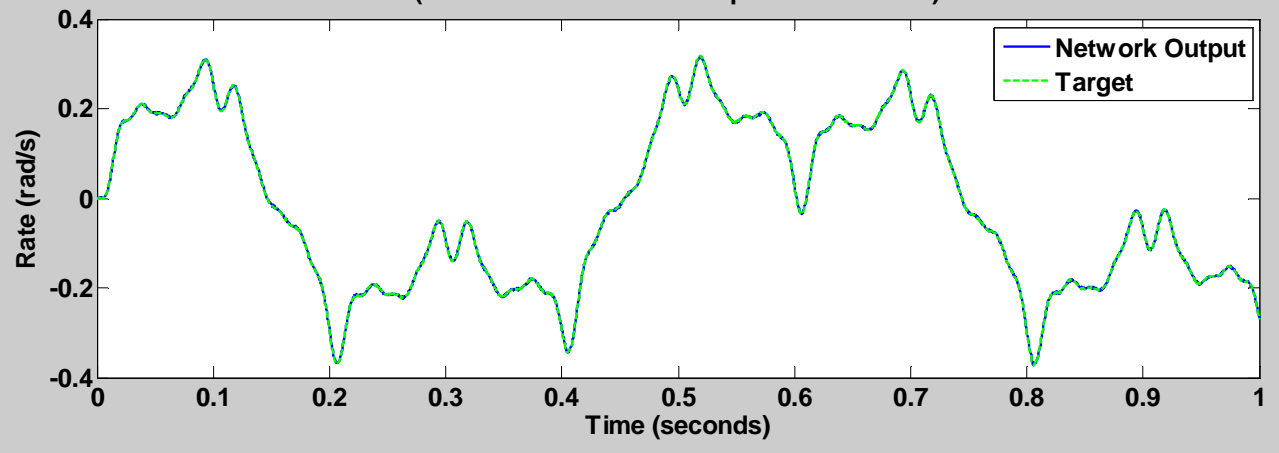

Figure 4.48: Simulation: Multiple Frequency Wave (Generalized Network)

The next input/target data set included the multiple frequency wave as input

(Figure 3.6). The simulations results are shown in Figure 4.48 above. The mean squared error was 2.2514E-6 and the R-squared value was 0.99993 . 

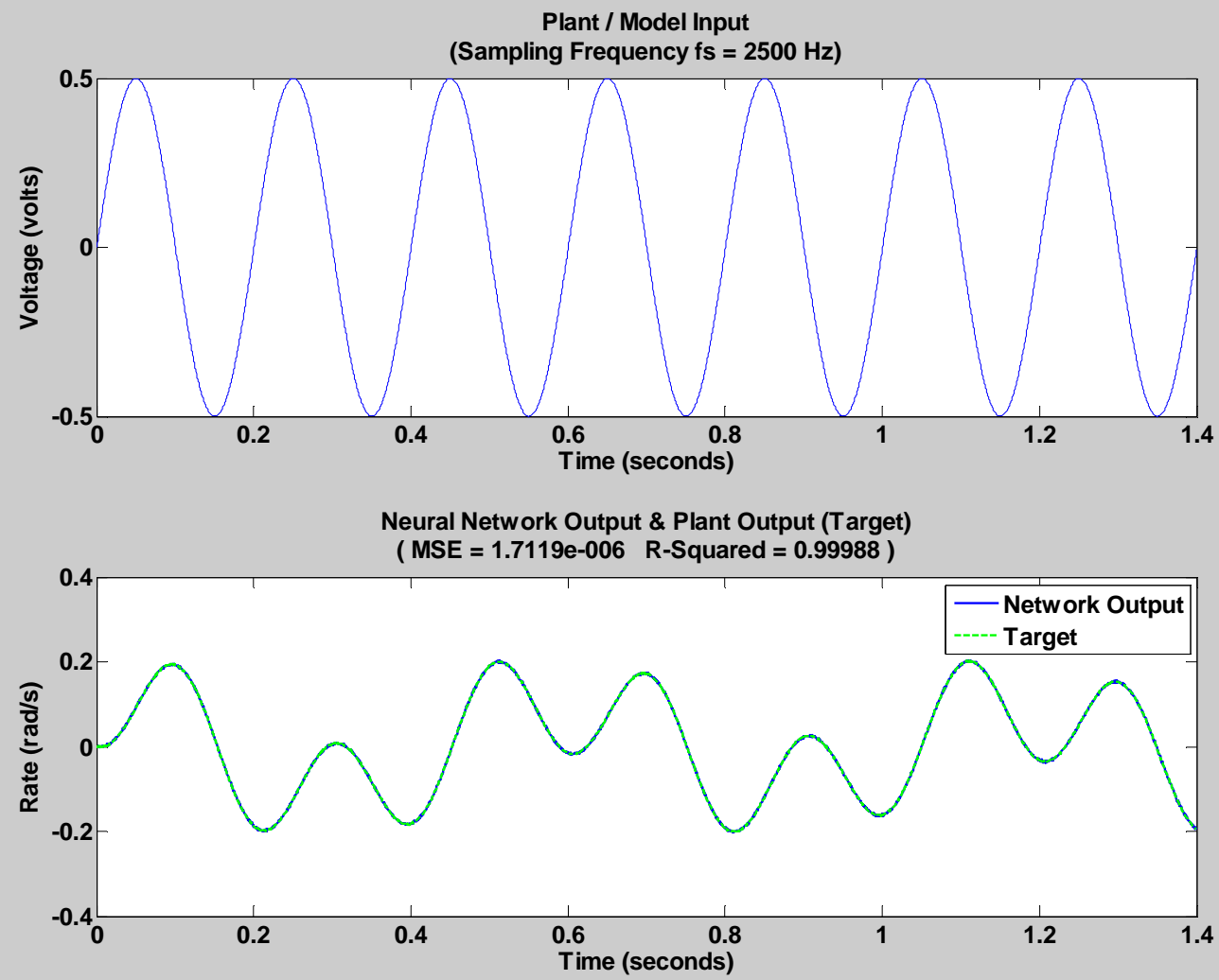

Figure 4.49: Simulation: $5 \mathrm{~Hz}$ Sine Wave (Generalized Network)

The next input/target pair included the $5 \mathrm{~Hz}$ sine wave as input and is shown in Figure 3.2. The simulations results are shown in Figure 4.49 above. The mean squared error was $1.7119 \mathrm{E}-6$ and the R-squared value was 0.99988 . 

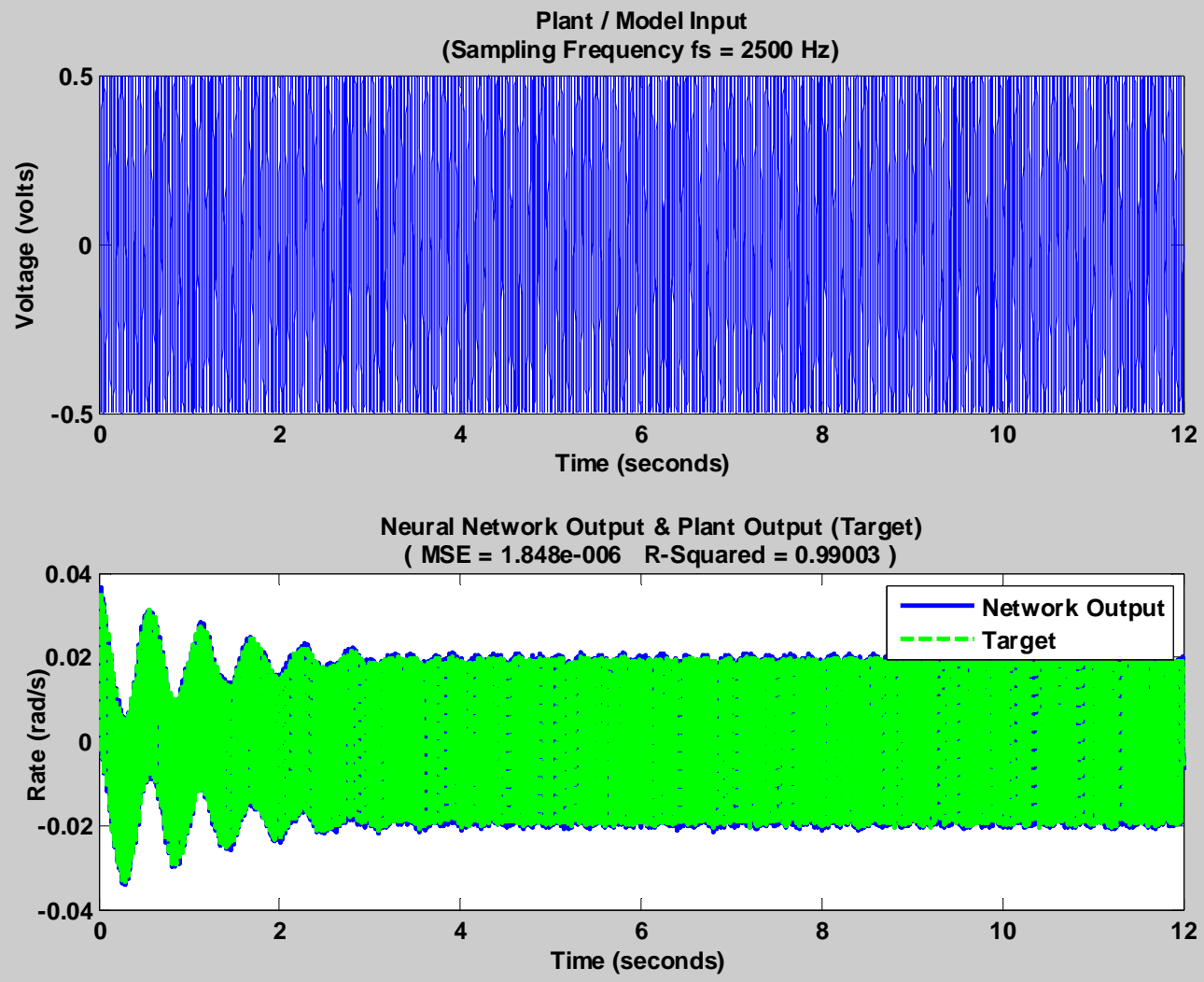

Figure 4.50: Simulation: $35 \mathrm{~Hz}$ Sine (Generalized Network) 

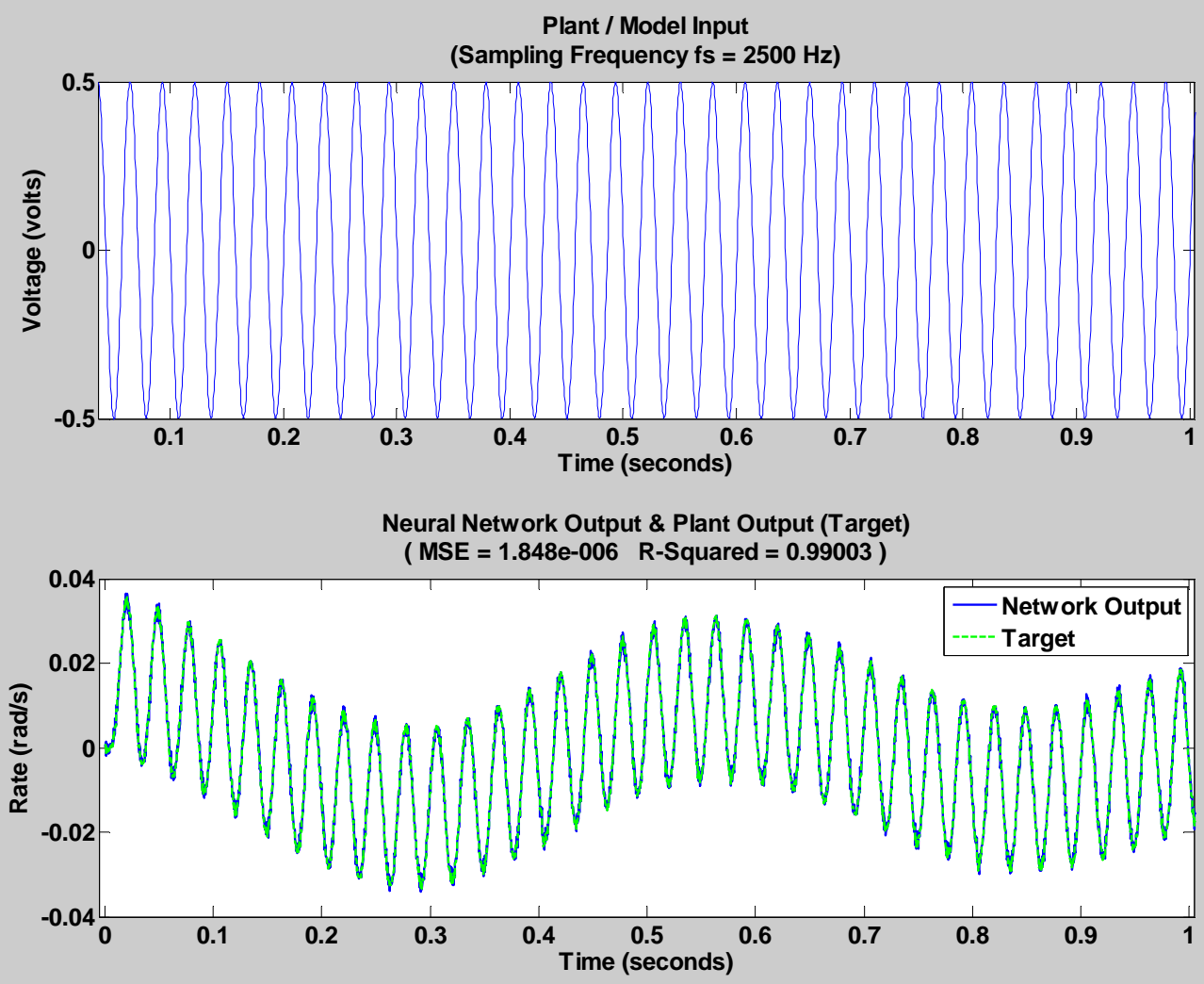

Figure 4.51: Simulation: 35 Hz Sine (Generalized Network) Zoomed

The $35 \mathrm{~Hz}$ sine wave input was used for the next simulation. The data measured from the real system is shown in Figure 3.4 through Figure 3.5 and the simulation results are shown in Figure 4.50 and Figure 4.51 above. The mean squared error was $1.848 \mathrm{E}-6$ and the R-squared value was 0.99003 . 
Plant I Model Input

(Sampling Frequency fs $=2500 \mathrm{~Hz}$ )

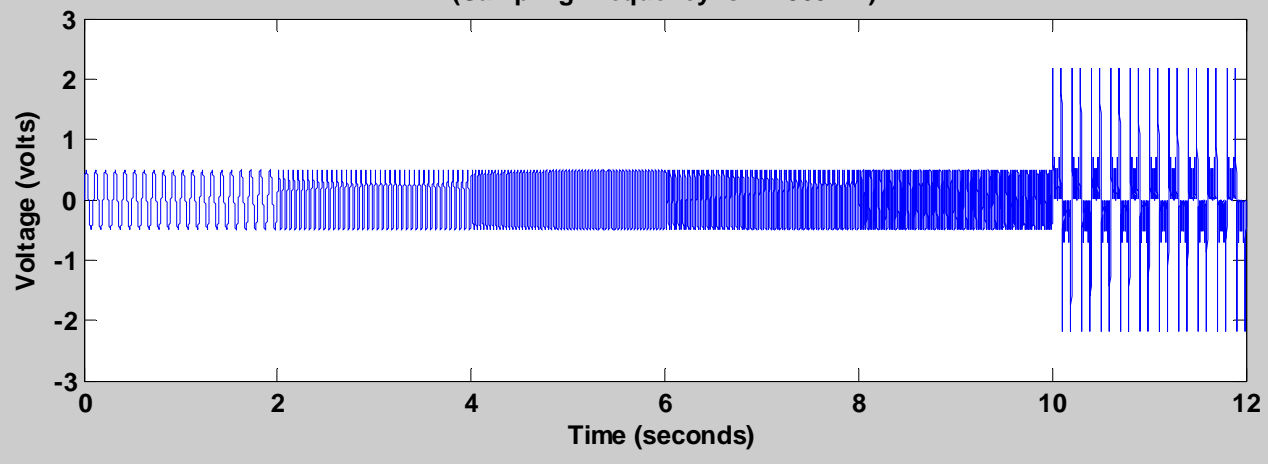

Neural Network Output \& Plant Output (Target)

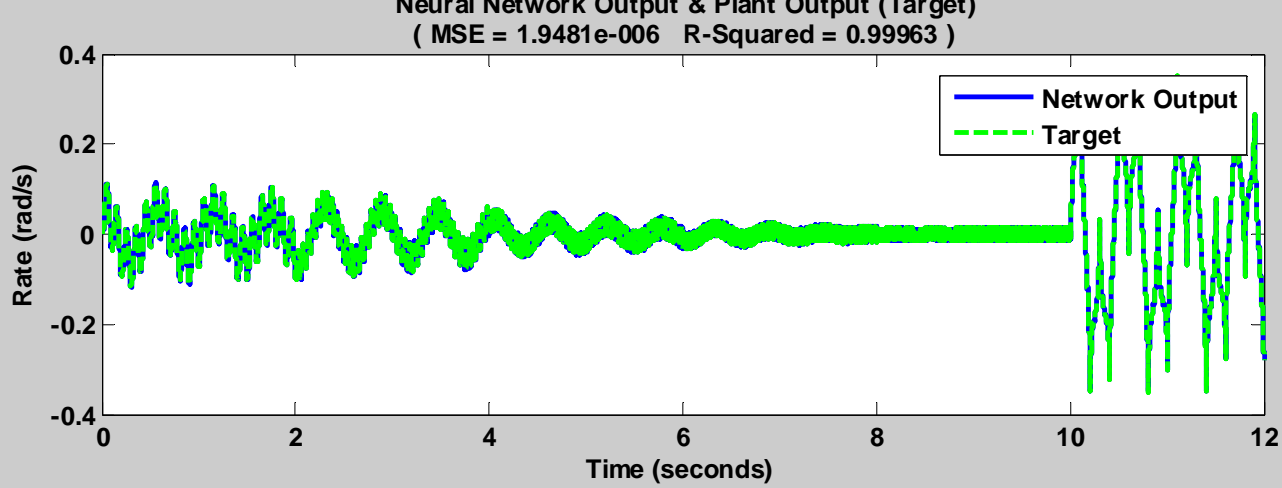

Figure 4.52: Simulation: Single \& Multiple Freq Waves (Generalized Network) 
Plant / Model Input

(Sampling Frequency fs $=2500 \mathrm{~Hz}$ )

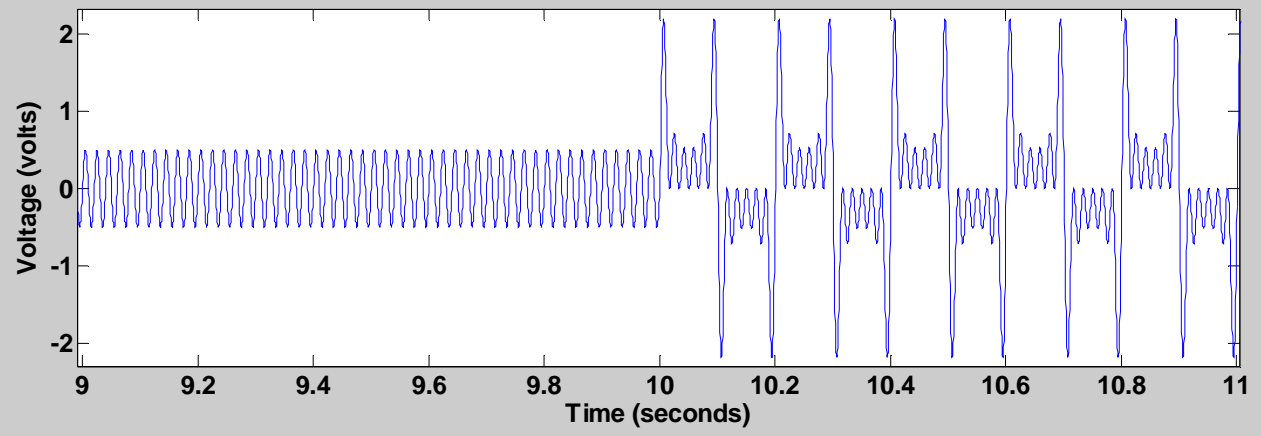

Neural Network Output \& Plant Output (Target)

( MSE = 1.9481e-006 R-Squared $=0.99963$ )

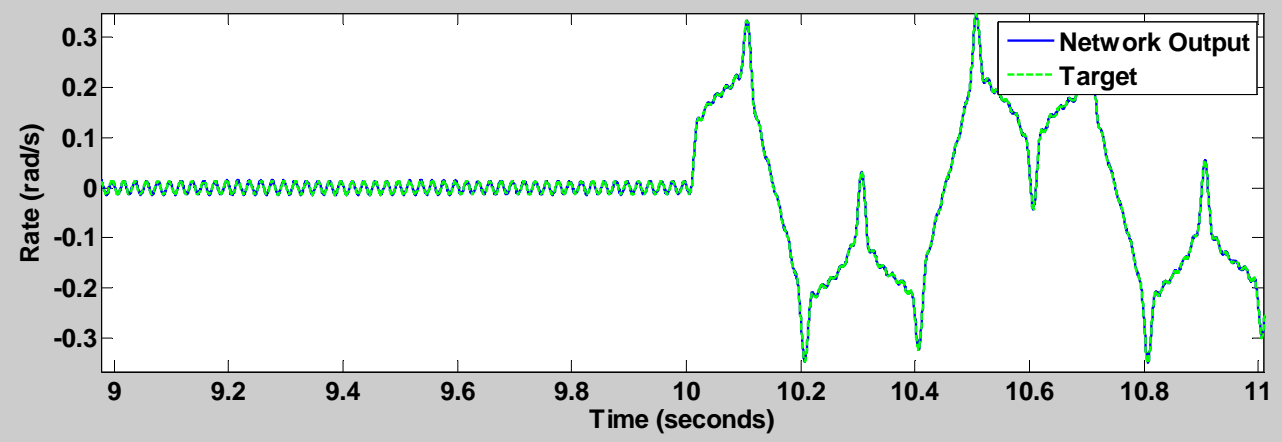

Figure 4.53: Simulation: Single \& Mult Freq Waves (Generalized Network) Zoomed

The next data set includes the input defined by multiple sine waves appended and a multiple frequency wave appended. The data measured from the actual system is shown in

Figure 3.7 and the simulation results are shown in Figure 4.52 and Figure 4.53 above. The mean squared error was $1.9481 \mathrm{E}-6$ and the R-squared value was 0.99963 . 

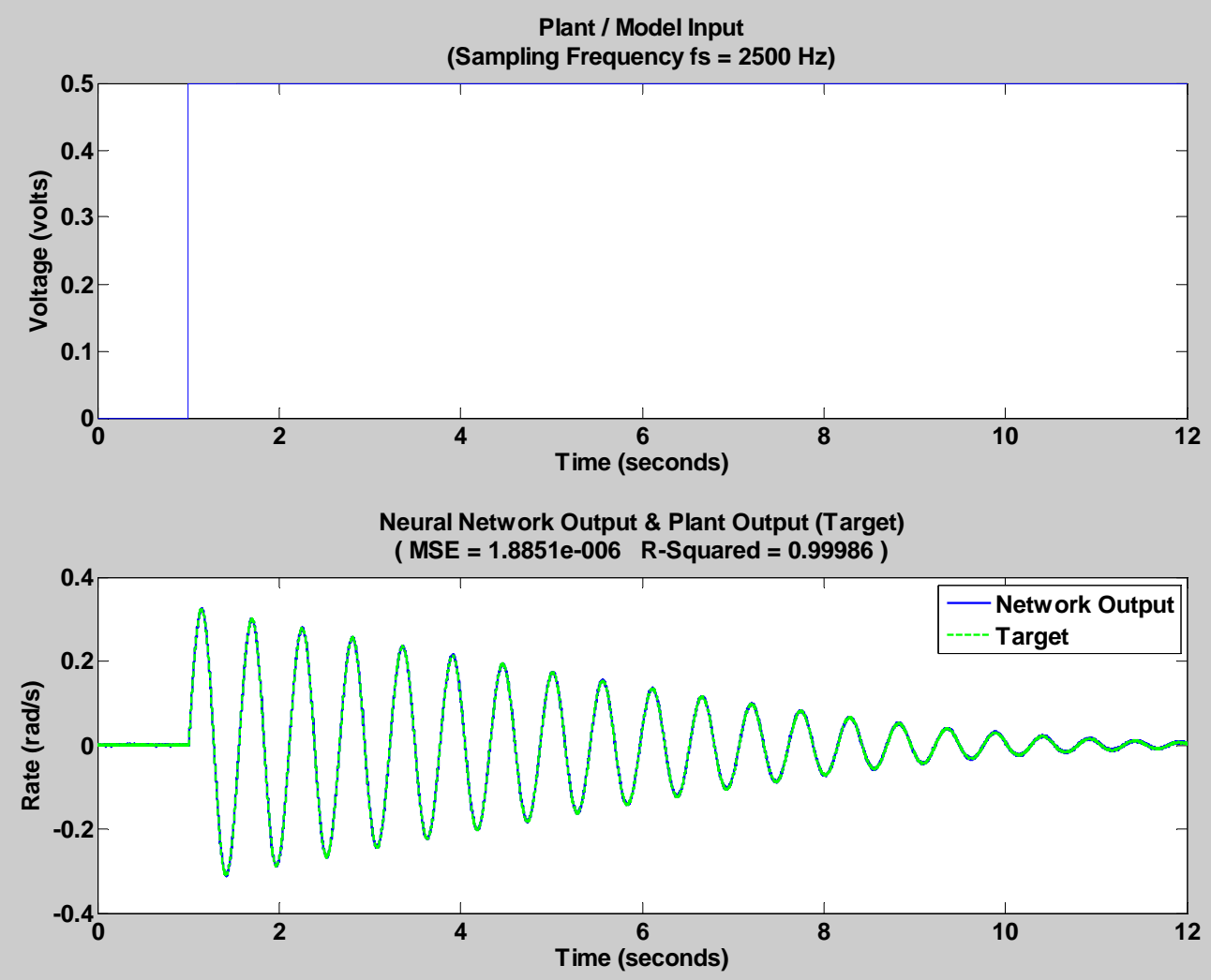

Figure 4.54: Simulation: Step Function (Generalized Network)

The data set using a step function as input is used for the last simulation. The measured system response is shown in Figure 3.19. The simulation results are shown in Figure 4.54 above. The mean squared error was $1.8854 \mathrm{E}-6$ and the R-squared value was 0.99986 .

The neural network was able to generalize well over the experimentally measured data. The input/output data pair which included a multiple frequency wave as input had the worst mean squared error with a value of $2.2514 \mathrm{E}-6$. However the worst R-squared value (0.99003) occured when simulating with the $35 \mathrm{~Hz}$ sine wave as input. 
Visually, the plotted simulation results looked very accurate. Only small aberrations could be seen when looking at the simulation results given a Chirp signal or $35 \mathrm{~Hz}$ sine wave as input.

To recap, the neural network used for the simulations was trained to the random signal (Figure 3.18) and reached MSE and R-squared values of 1.9801E-6, and 0.99997 respectively. A summary of the results is shown in Table 4.5 below.

Table 4.5: Network Generalization Simulation Results

\begin{tabular}{|l|c|c|}
\hline \multicolumn{1}{|c|}{ Input/Target Data Type } & MSE & R-Squared \\
\hline Chirp Signal & $1.9082 \mathrm{E}-6$ & 0.9921 \\
\hline Multiple Frequency Signal & $2.2514 \mathrm{E}-6$ & 0.99993 \\
\hline 5 Hz Sine Wave & $1.7119 \mathrm{E}-6$ & 0.99988 \\
\hline 35 Hz Sine Wave & $1.848 \mathrm{E}-6$ & 0.99003 \\
\hline Single \& Multiple Frequency Sine Waves & $1.948 \mathrm{E}-6$ & 0.99963 \\
\hline Step Function & $1.8854 \mathrm{E}-6$ & 0.99986 \\
\hline
\end{tabular}




\section{Chapter 5: Traditional vs. Neural Network Model Comparison}

System identification with neural network models is compared to a traditional method of modeling a gyro-stabilized camera system. First, using the traditional method, a model is created. Second, the model's output is simulated using the same input data sets used to simulate the neural network model output in section 4.4. Third, the traditional model output was compared to the real system output for each data set by calculating the mean squared error (MSE) and R-squared value. Lastly, the traditional model was compared to the neural network model by comparing the MSE and R-squared values.

\section{1 - Traditional Model}

A traditional method for system identification is to model the plant assuming a linear system. The model is created and simulated in Matlab Simulink. The block diagram for a traditional inner azimuth gimbal model is again shown in Figure 5.1 below.

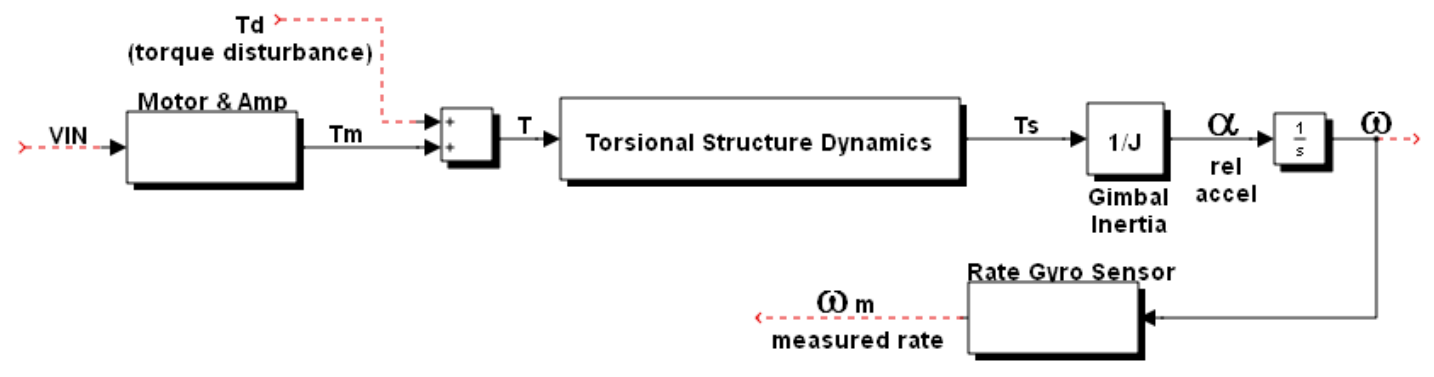

Figure 5.1: Traditional Model

The Structural Dynamics (as referenced in the above figure) are generally represented as a superposition of single degree of freedom (SDOF) linear models. The transfer function 
of each of these models is typically derived from FEA (finite element analysis) or modal analysis. FEA is accomplished through extensive computer simulation and modal analysis is typically executed using accelerometers and special computer software and equipment. Both of these options were not fully available at the time this paper was written. The finite element analysis was available in limited form for a small portion of the structure. When implemented the analysis indicated small oscillations at frequencies in the thousands of Hz. Since the rate of sensor measurement in the system under study is $2500 \mathrm{~Hz}$ (Nyquist frequency of $1250 \mathrm{~Hz}$ ) it can be argued that the system can be modeled to within an acceptable level by assuming the structure is rigid. Using this assumption the system can now be modeled as shown in the figure below.

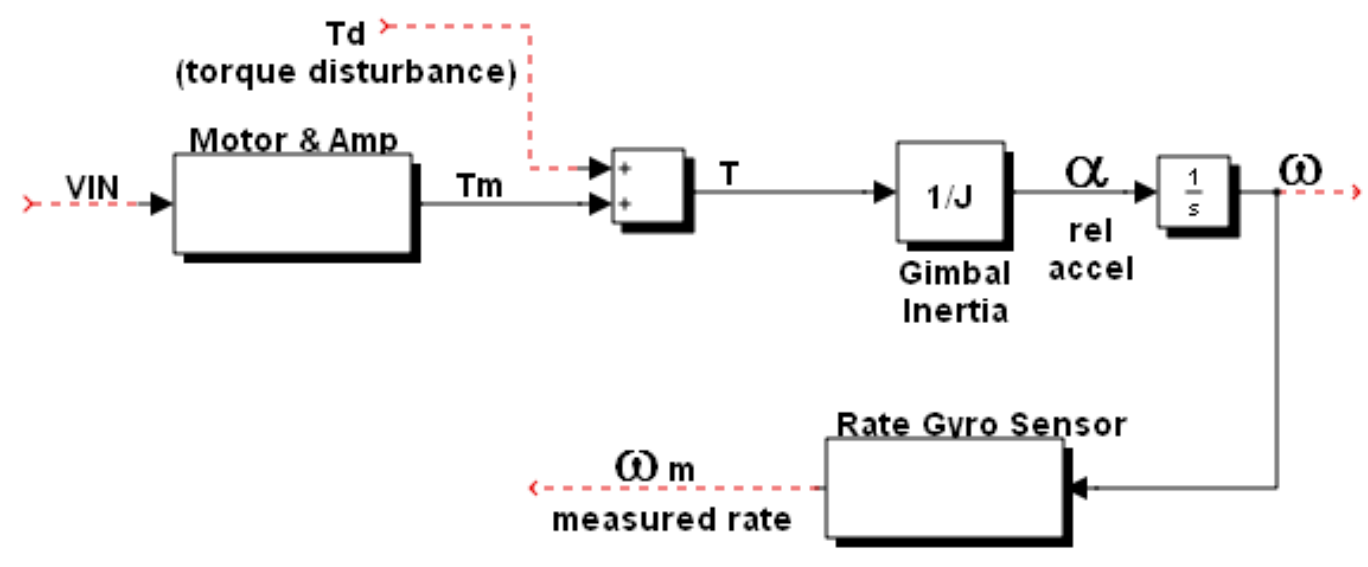

Figure 5.2: Inner Azimuth Gimbal (Rigid Structure)

The input VIN is a voltage and the output $\omega_{m}$ is measured relative (between inner and outer gimbal) angular rate in radians/s (outer gimbal assumed stationary in inertial space). 
The torque disturbance $T_{d}$ in the above figure consists mostly of a spring torque created by a flexural pivot bearing or flex pivot (see Figure 1.9). It is important to note that the flex pivot is used in place of a standard roller bearing in order to remove nonlinearities associated with this type of bearing. Thus, friction torques in this particular gimbal can be assumed sufficiently small and thus can be ignored. The flex pivot accounts for a large majority of the disturbance torque; applies a torque in the opposite direction the motor torque, and is related proportionally to the position of the inner gimbal (relative to outer). Other torques such as gyroscopic torque (created by the gyro rate sensor), on-board shaking forces, and cable torques are very hard to model. A portion of these torques can be taken into account by assuming they sum to equal a "dampening" torque. Assuming the only torque disturbance is due to the spring torque and a dampening torque then $T_{D}=T_{s}+T_{d}=-K_{s} \Delta \theta-c \dot{\theta}$ where $T_{s}$ is the spring torque, $T_{d}$ is a "dampening" torque, $K_{S}$ is the spring constant, $\Delta \theta$ is the relative angular displacement between inner and outer azimuth gimbal, c is the system damping, and $\dot{\theta}$ is angular velocity. The damping can be defined as $c=\zeta C_{c r}=\zeta\left(2 \sqrt{K_{s} \cdot J}\right)$ where $C_{c r}$ is called the critical damping, $\zeta$ is the damping coefficient, $K_{S}$ is the spring constant, and $J$ is the inertial Tensor (moment of inertia). The damping coefficient is defined as $\zeta=\frac{\ln \left(\frac{u_{1}}{u_{2}}\right)}{2 \pi}$, where $u_{1}$ is the amplitude of a spring mass oscillation in period one, $u_{2}$ is the amplitude in period two. Included in the equation for damping results is $c=\zeta C_{c r}=\frac{\ln \left(\frac{u_{1}}{u_{2}}\right)}{2 \pi}\left(2 \sqrt{K_{S} \cdot J}\right)$. Note that the negative signs in the equation for $T_{D}$ are present because the spring applies a torque in the opposite direction (as mentioned above) 
the motor torque at all times. Given the above equation for $T_{D}$ the model can be again redrawn as shown in the figure below.

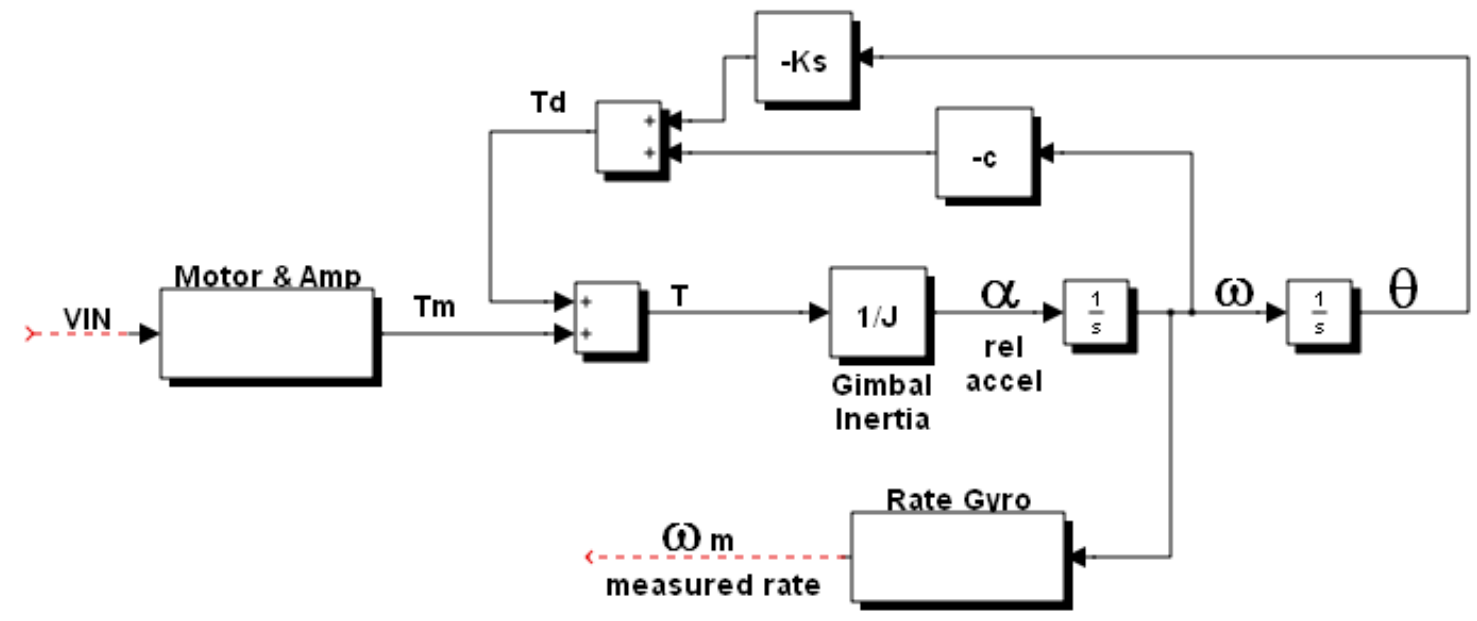

Figure 5.3: Gimbal (Rigid Structure w/ Spring \& Dampening Torque Disturbances)

To obtain the spring constant $K_{s}=\frac{T_{d}}{\Delta \theta}$, a known torque $-T_{d}$ was applied to the inner azimuth axis while the angular displacement $\Delta \theta$ at steady state was measured. This was accomplished by pointing the gimbal toward the ground and hanging weights on the edge of the inner azimuth gimbal. The torque was calculated based on the weight and its distance from the flex pivots (gimbal pivot points). The corresponding angles for a given weight were measured using the Axsys "PC Scope" (also called "Gimbal Tool") program. Several data points were taken. A plot of torque vs. angular displacement is shown in Figure 5.4 below. 


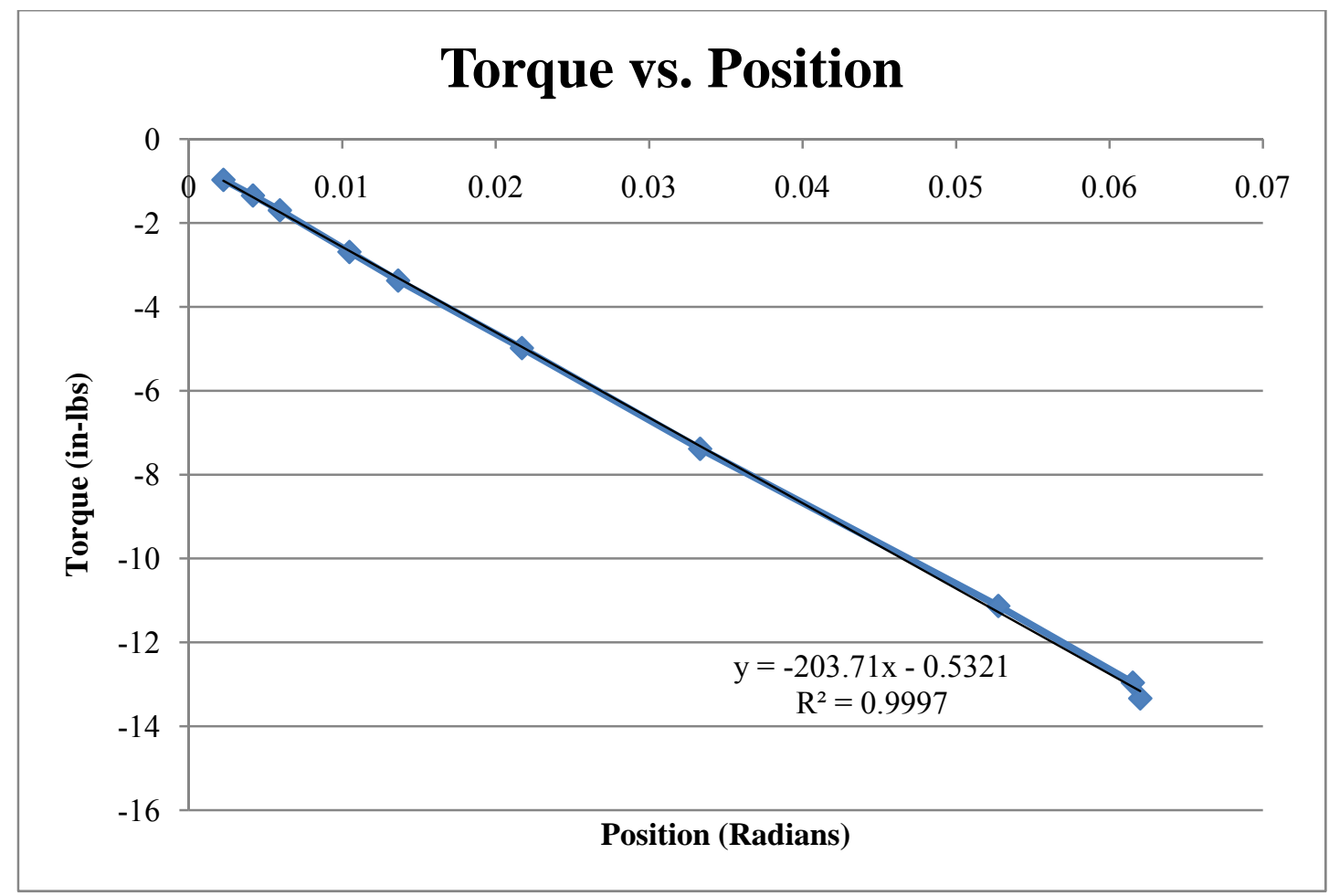

Figure 5.4: Spring Torque vs. Angle

It can be seen from the above plot that $K_{s} \approx-203 \frac{i n-l b s}{r a d}$. This number coincides with the spring constant of the flex pivots $\left(\approx-86 \frac{i n-l b s}{r a d} \times 2\right.$ flex pivots $\left.\approx-172 \frac{i n-l b s}{r a d}\right)$. It is important to note the bias of -.53 in-lbs in the above figure. This could be caused by a static imbalance of the inner gimbal, angle offset of the flex pivots, or another spring torque acting on the gimbal.

The inertia Tensor, $J$, and dampening, c, can be calculated by measuring the oscillation frequency and rate of dampening after applying a step function to the system. A plot of angular displacement vs. time is shown in Figure 5.5 below given a step input of $\mathrm{VIN}=.5 \mathrm{~V}$. 


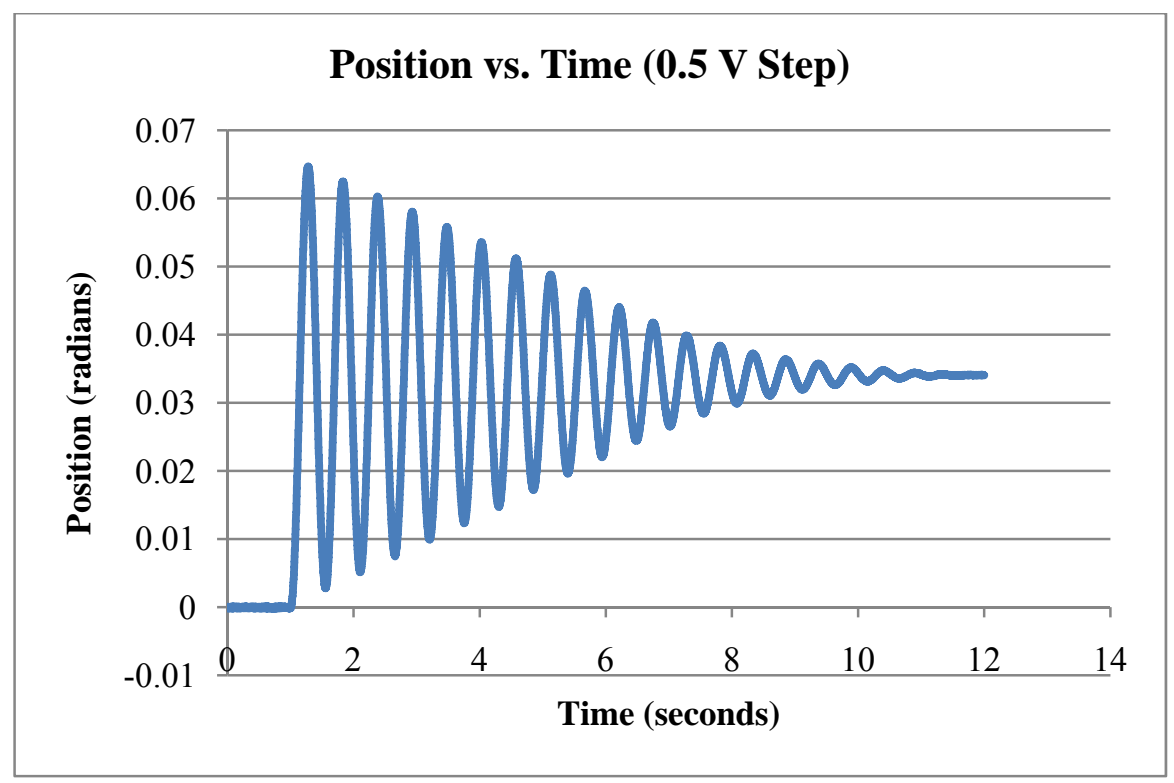

Figure 5.5: Angular displacement vs. Time for a .5 Volt Step Input

The inertia tensor $\mathrm{J}$ (moment of Inertia) in Figure 5.3 can be calculated using the equation for the period of a mass-spring system $T=2 \pi \sqrt{\frac{m}{k}}$. For rotational motion of a torsion oscillator, the moment of inertia $\mathrm{J}$ replaces the mass $\mathrm{m}$, and the torsion constant $K_{S}$ replaces the spring constant $\mathrm{k}$ from Hooke's law. Therefore, $T=2 \pi \sqrt{\frac{J}{K_{S}}}$. Calculating for $\mathrm{J}$, results in $J=K_{S}\left(\frac{T}{2 \pi}\right)^{2}$, where T is the oscillation period (seconds), and $K_{S}$ is the torsion constant $\left(\frac{\text { in-lbs }}{\text { radian }}\right)$. The period $\mathrm{T}$ is measured from Figure 5.5 above as $\mathrm{T}=.56$ seconds. Using this period and the torsion constant, $K_{S}$ calculated previously, the inertia tensor can be calculated as: $J=K_{s}\left(\frac{T}{2 \pi}\right)^{2}=203 \frac{\mathrm{in}-\mathrm{lbs}}{\mathrm{rad}}\left(\frac{.56 \mathrm{sec}}{2 \pi}\right)^{2}=1.6125 \frac{\mathrm{in}-\mathrm{lbs}-\mathrm{sec}^{2}}{\mathrm{rad}}$.

Using the measurement in Figure 5.5 the dampening can now be calculated. Again, the dampening is defined as $c=\zeta C_{c r}=\frac{\ln \left(\frac{u_{1}}{u_{2}}\right)}{2 \pi}\left(2 \sqrt{K_{s} \cdot J}\right)$. The values for $u_{1}$ and 
$u_{2}$ are found by measuring the amplitude of the first two oscillations in Figure 5.5 above:

$u_{1}=.06463$ and $u_{2}=.06256$. The dampening value is thus

$c=\frac{\ln \left(\frac{.06463}{.06256}\right)}{2 \pi}\left(2 \sqrt{203 \frac{\mathrm{in}-l b s}{\mathrm{rad}} \cdot 1.6125 \frac{\mathrm{in}-l b s-s^{2}}{\mathrm{rad}}}\right)=0.18747 \frac{\mathrm{in}-\mathrm{lbs}-\mathrm{sec}}{\mathrm{rad}}$.

The motor/amplifier block shown in Figure 5.3 consists of a current amplifier (implemented as an analog circuit) which drives a "torqer" motor consisting of a coil and magnet. The coil is attached to the outer gimbal where as the magnet is attached to the inner gimbal. The motor/amplifier block can be modeled as shown in the figure below.

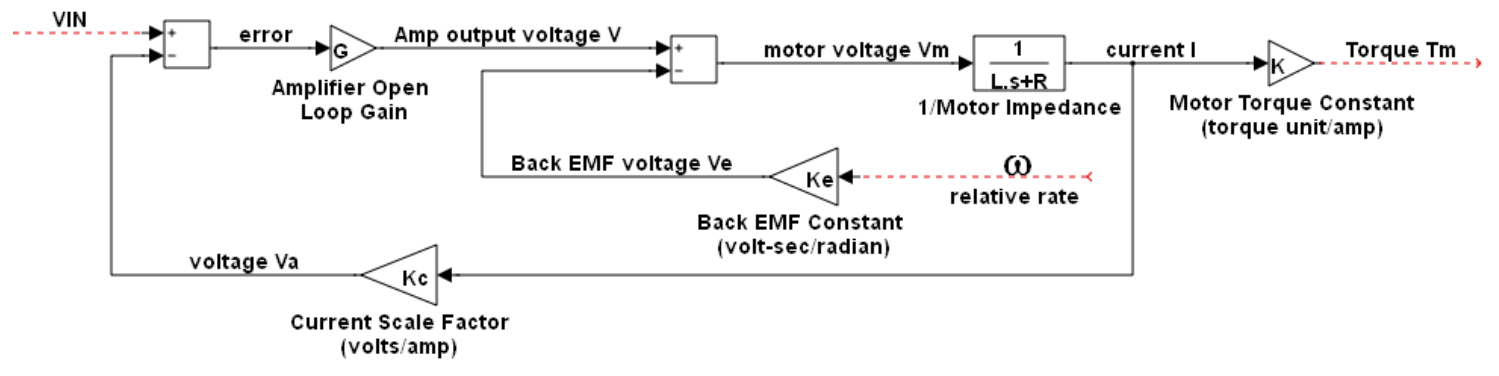

Figure 5.6: Amplifier/Motor Model

The amplifier open loop gain in the above figure is defined as $G=\frac{6(.754 E-3 s+1)}{0.0158 E-6 s^{2}+.168 E-3 s}$.

The gain $\mathrm{G}$ was calculated based on the analog circuit. The current scale factor $K_{c}=1$, motor inductance $L=4.23 \mathrm{mH}$, and motor resistance $R=10.1 \mathrm{ohms}$. The motor torque constant can be calculated as $K=\frac{T_{m}}{I}$ where $T_{m}$ is the motor output torque and $I$ is the input current to the motor. Since the flex pivot applies a torque in the opposite direction the motor torque the two torques are equal in magnitude at a steady state position. To obtain $T_{m}$ the steady state angular displacement $\Delta \theta$ is measured using the data obtained in Figure 5.5. $T_{m}$ is then calculated as: $T_{m}=-T_{d}=-K_{s} \Delta \theta-c \dot{\theta}$. Since at steady state 
$\dot{\theta}=0 \frac{\mathrm{rad}}{\mathrm{s}}$ then $T_{m}=-K_{s} \Delta \theta$. Given the data shown in the above plot the steady state angular displacement was measured as .034 radians. The torque can now be calculated as $T_{m}=-K_{s} \Delta \theta=-\left(-203 \frac{\mathrm{in}-\mathrm{lbs}}{\mathrm{rad}}\right)(.0352$ radians $)=7.146 \mathrm{in}-$ lbs. The motor torque constant can now be calculated as $K_{t}=\frac{T_{m}}{I}=\frac{7.146 \mathrm{in}-\mathrm{lbs}}{.5 \mathrm{amps}}=14.29 \frac{\mathrm{in}-\mathrm{lbs}}{\mathrm{amp}}$. The back EMF constant of the motor $K_{e}$ with units of $\frac{v o l t}{\left(\frac{r a d}{\sec }\right)}$ is equal to the motor torque constant $K_{t}$ with units of $\frac{N-m}{a m p}$. Using the value of the torque constant calculated above: $K_{t}=\left(\frac{14.29 \mathrm{in}-\mathrm{lbs}}{a m p}\right)\left(\frac{1 \mathrm{~N}-\mathrm{m}}{8.850732 \mathrm{in}-\mathrm{lbs}}\right)=1.615 \frac{\mathrm{N}-\mathrm{m}}{\mathrm{amp}}$. Thus, $K_{e}=1.615 \frac{\text { volt }}{\left(\frac{\mathrm{rad}}{\mathrm{sec}}\right)}$ or $\frac{\text { volt }-\mathrm{sec}}{\mathrm{rad}}$. The gyro rate sensor can be modeled as shown in the figure below.

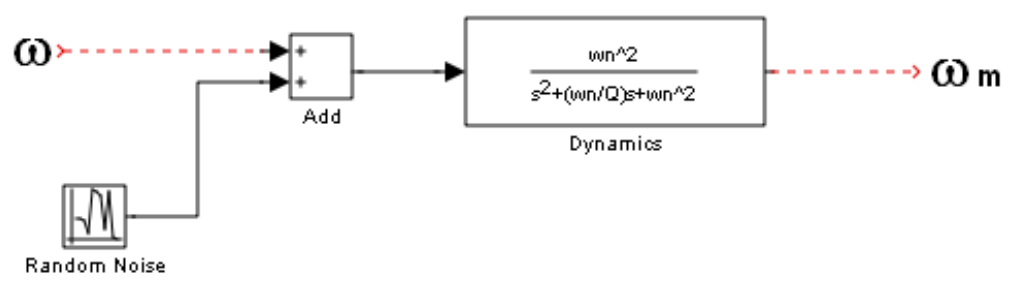

Figure 5.7: Gyro Rate Sensor

In the above figure the corner frequency of the gyro $\omega_{n} \cong 628 \frac{\mathrm{rad}}{\mathrm{s}}$ and the quality factor $Q \cong 10$. The random noise shown in the figure above is typically modeled as a Gaussian white noise. To increase accuracy this noise was instead generated by measuring the gyro rate sensor while it remains motionless. This measured gyro signal is displayed in the Figure 5.8 and Figure 5.9 below. 


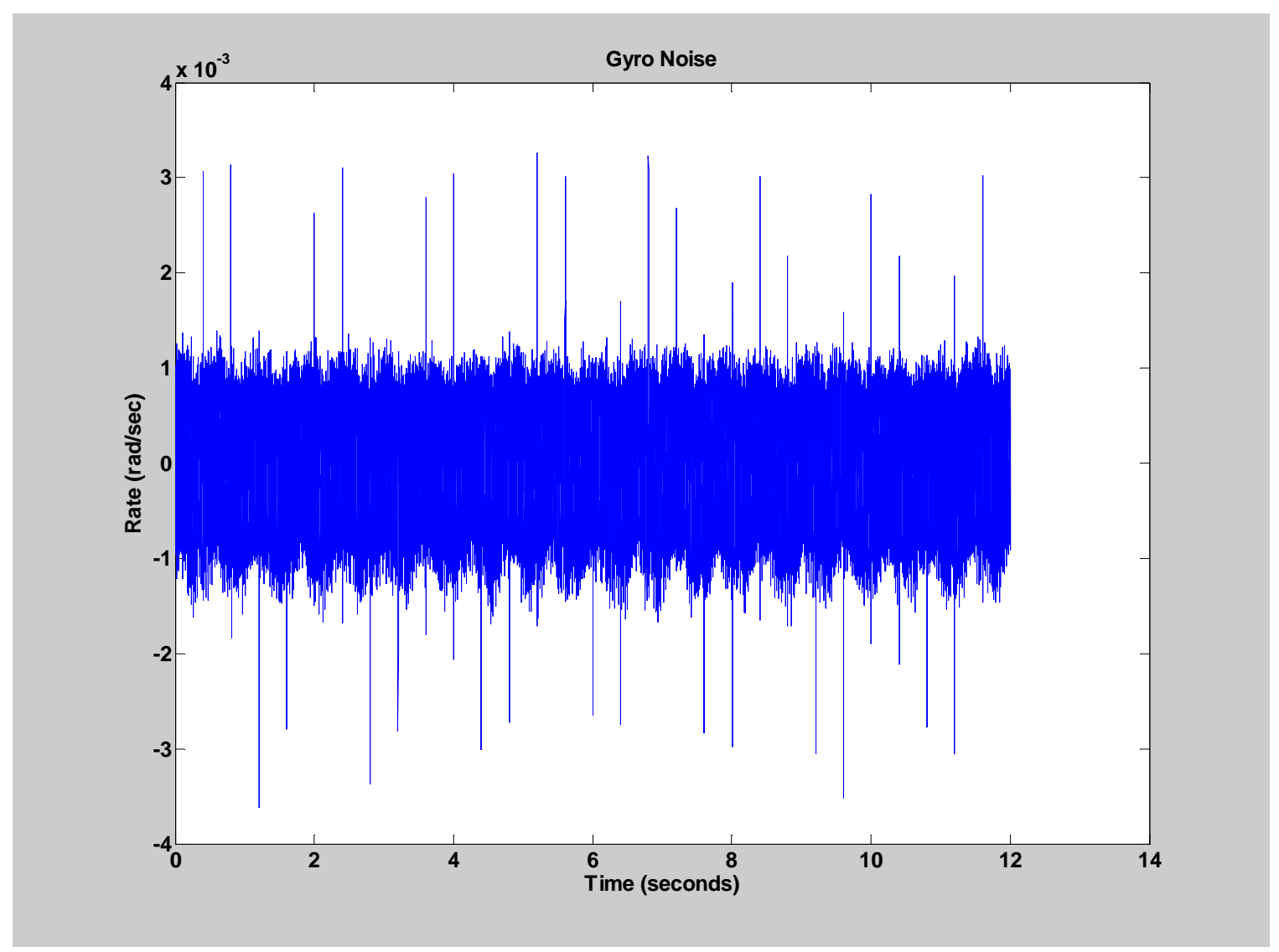

Figure 5.8: Measured Gyro Rate Sensor Noise 


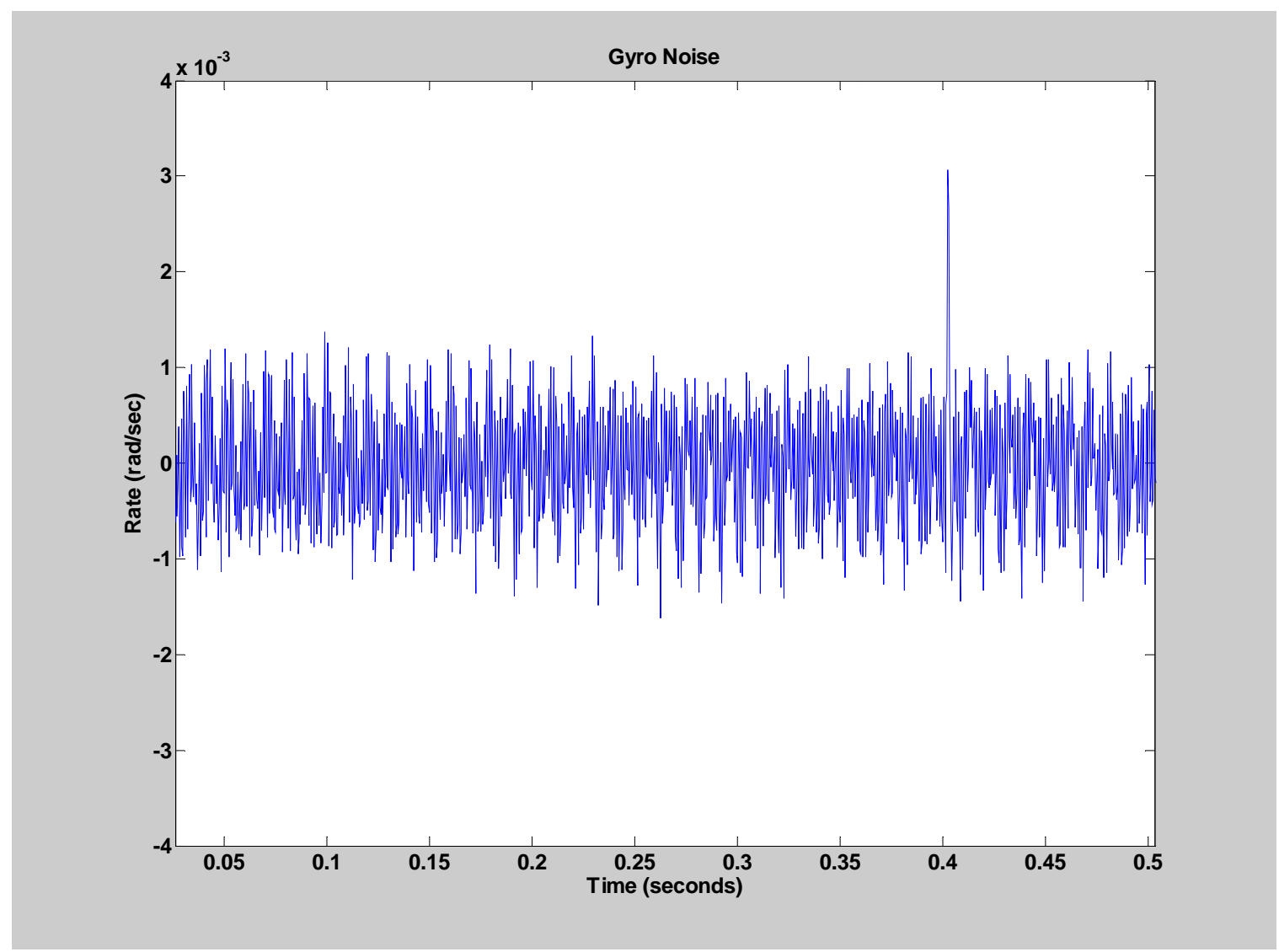

Figure 5.9: Measured Gyro Rate Sensor Noise (Zoomed)

The traditional model was generated and simulated in Matlab Simulink. The model is shown below with all constants included.

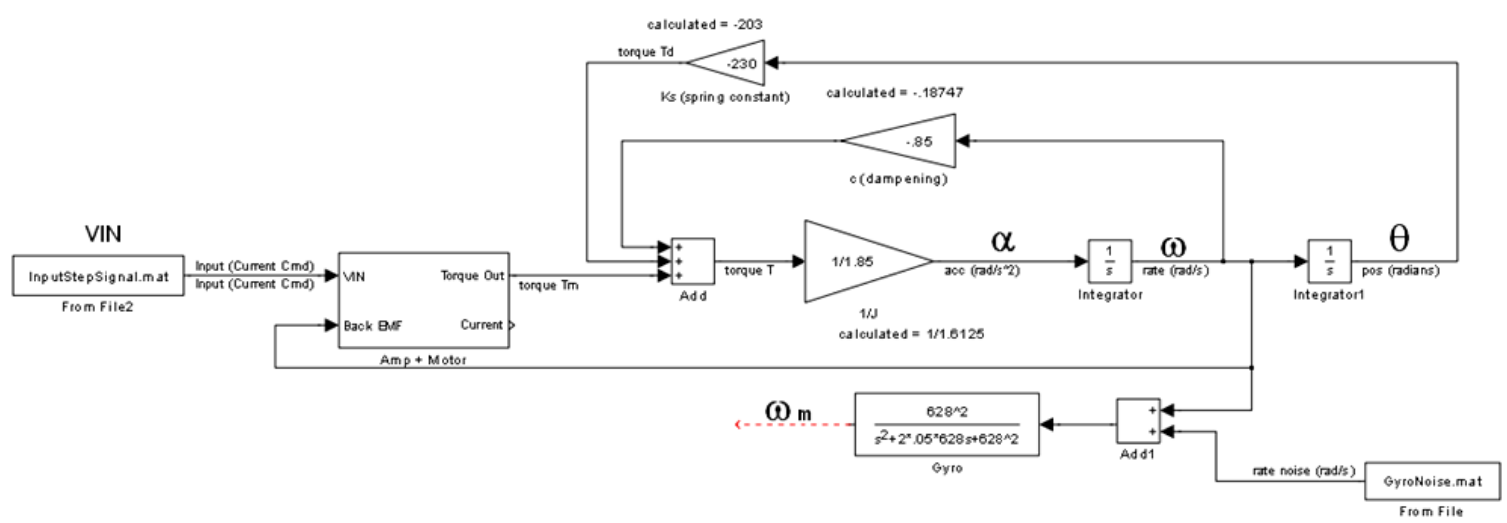

Figure 5.10: Simulink Traditional Model 
It is important to note that the inertial tensor constant $J$ was adjusted from the calculated value of 1.6125 to $2 \frac{i n-l b s-s e c^{2}}{r a d}$, the flex pivot spring torque constant $K_{s}$ was adjusted from the calculated value of 203 to $230 \frac{i n-l b s}{r a d}$ and the damping value $\mathrm{c}$ from 0.18747 to 0.85. This was done to increase the accuracy of the model and was accomplished through a basic understanding of how the constants affect the response and through trial and error. The values were adjusted based on the following assumptions: an increase in spring constant $K_{s}$ magnitude causes a decrease in rate output magnitude; an increase in inertia Tensor $\mathrm{J}$ causes a decrease in oscillation frequency, and an increase in damping $\mathrm{c}$ causes a more rapid decrease in magnitude over time (more damping). The Step function data set (Figure 3.19) was used to test the response while adjusting the above values.

The motor constants are entered into Simulink by double clicking on the Amp + Motor block in Figure 5.10 above. The window that appears and the parameters entered is shown in the figure below.

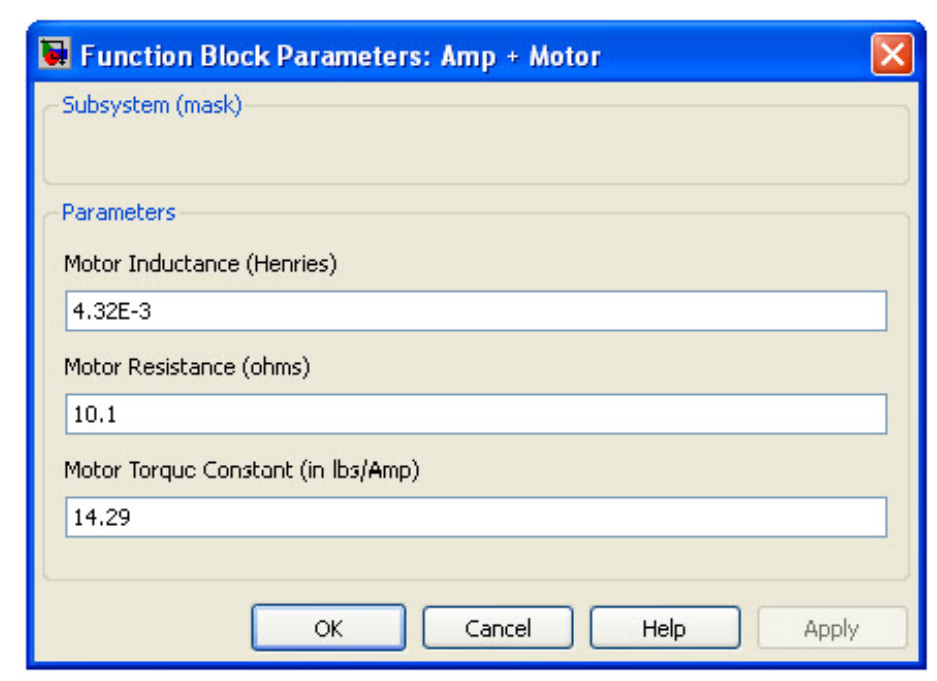

Figure 5.11: Simulink Motor Constants 
The Simulink model for the amplifier and motor block in Figure 5.10 is presented in

Figure 5.12 below.

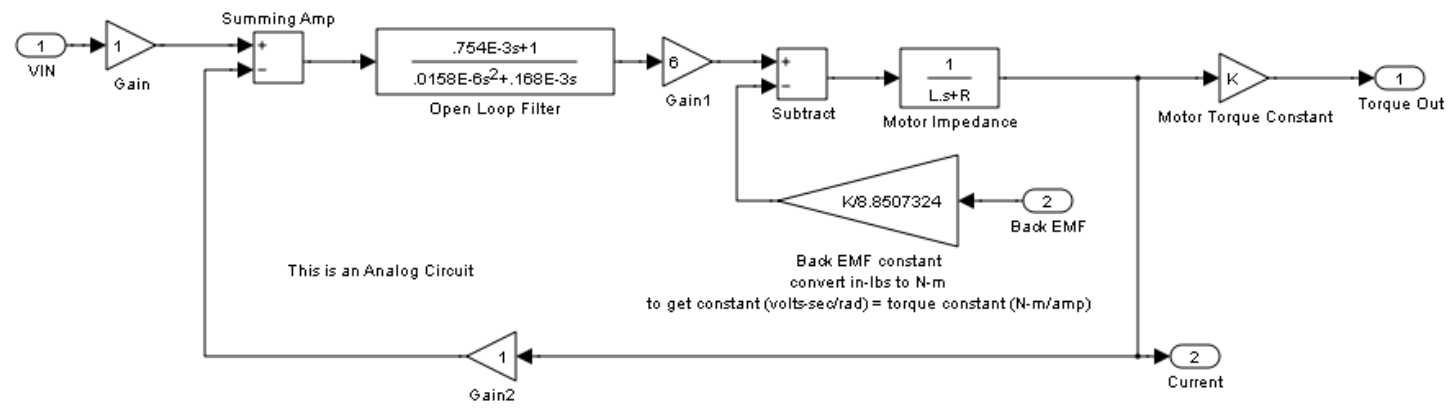

Figure 5.12: Simulink Amplifier and Motor Model 


\section{2 - Traditional Model Simulation}

The traditional model was simulated using the same real system data used when simulating the neural network model in section 4.4. Using the simulation results a mean squared error and R-squared value is calculated by comparing the traditional model output with the actual measured output. Seven data sets are used: Random signal, Chirp Signal, Multiple Frequency signal, $5 \mathrm{~Hz}$ sine wave, $35 \mathrm{~Hz}$ sine wave, single and multiple frequency, and a Step function. The simulation results are shown below.
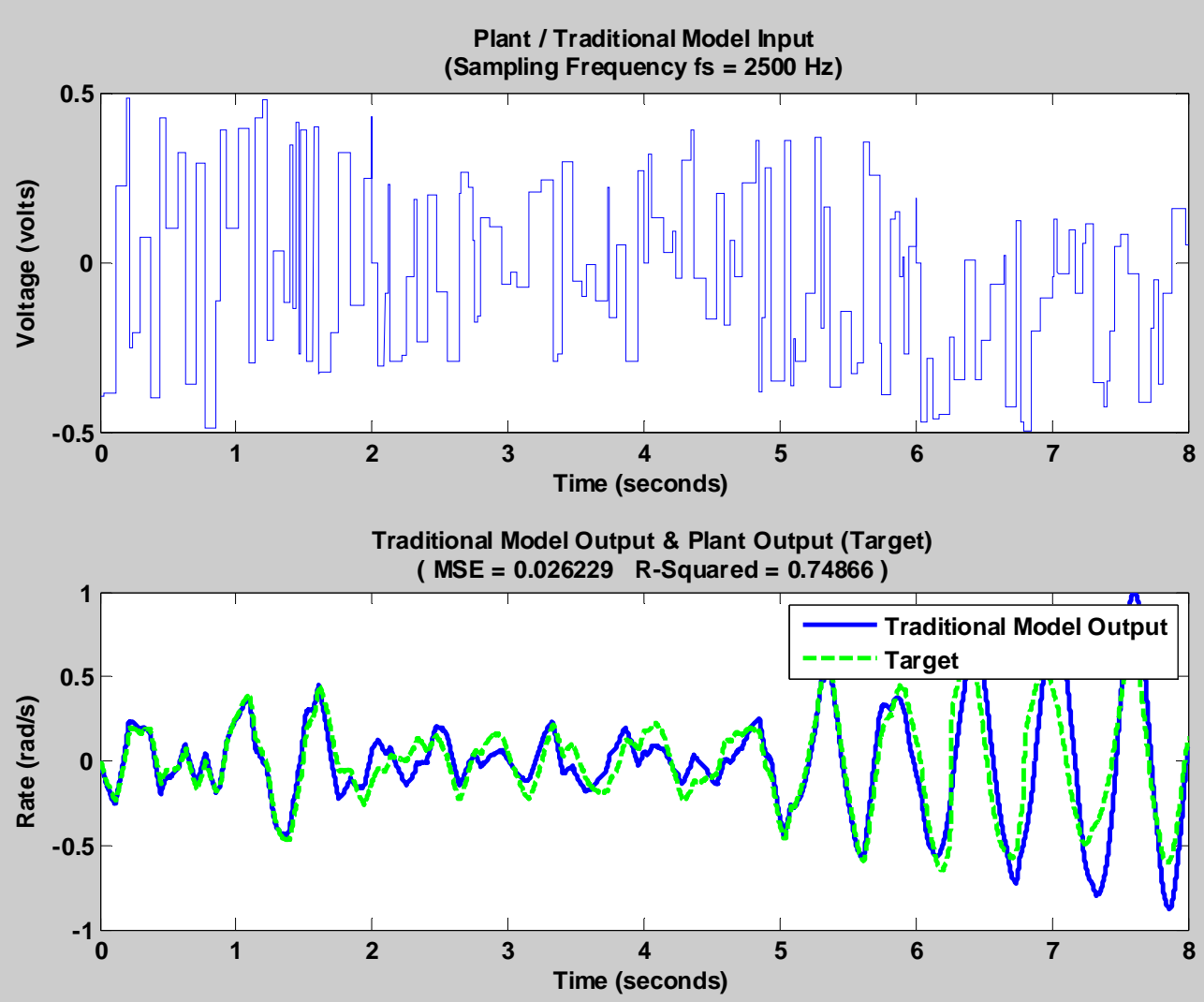

Figure 5.13: LTI Model Simulation Results (Random Signal) 


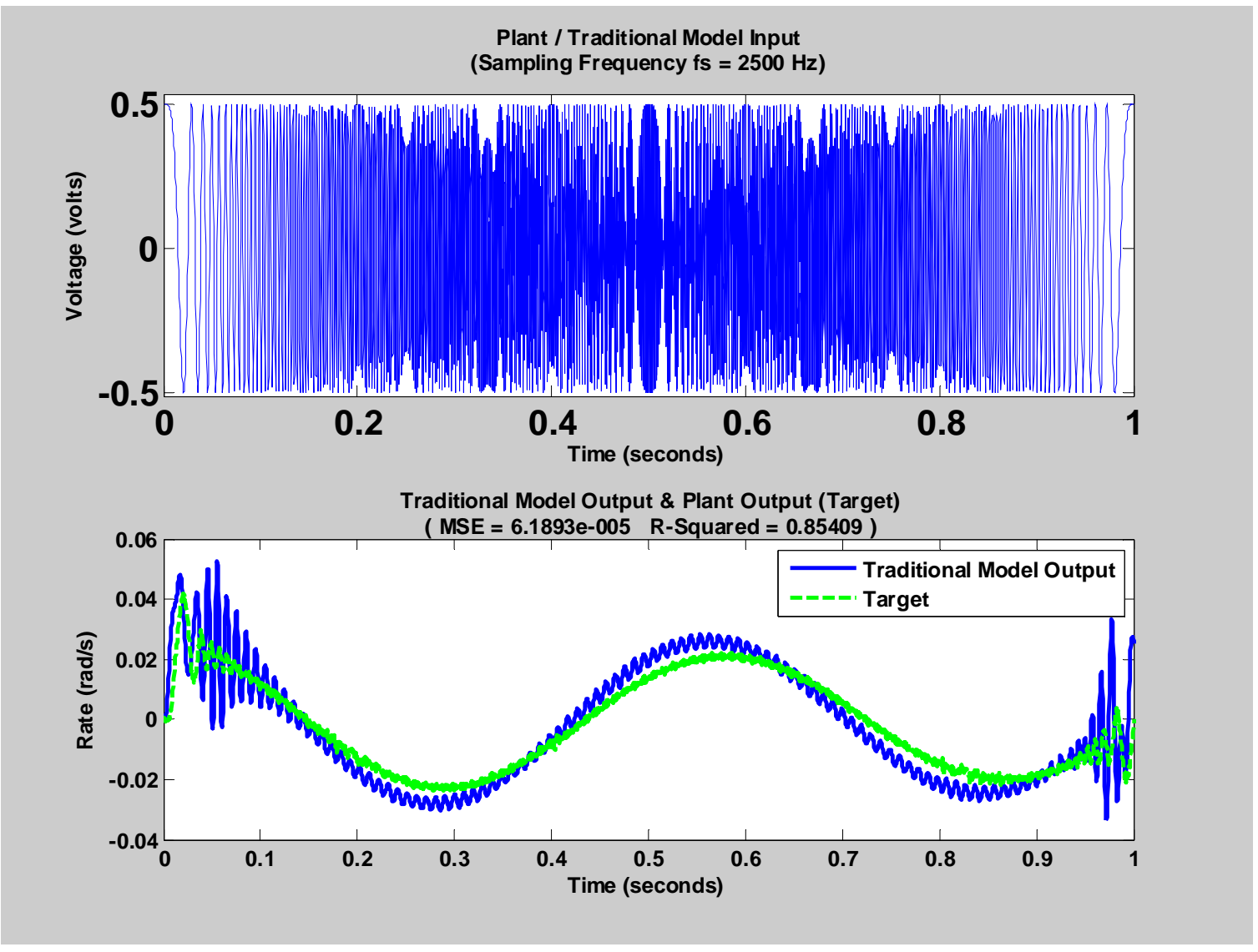

Figure 5.14: LTI Model Simulation Results (Chirp Signal) 


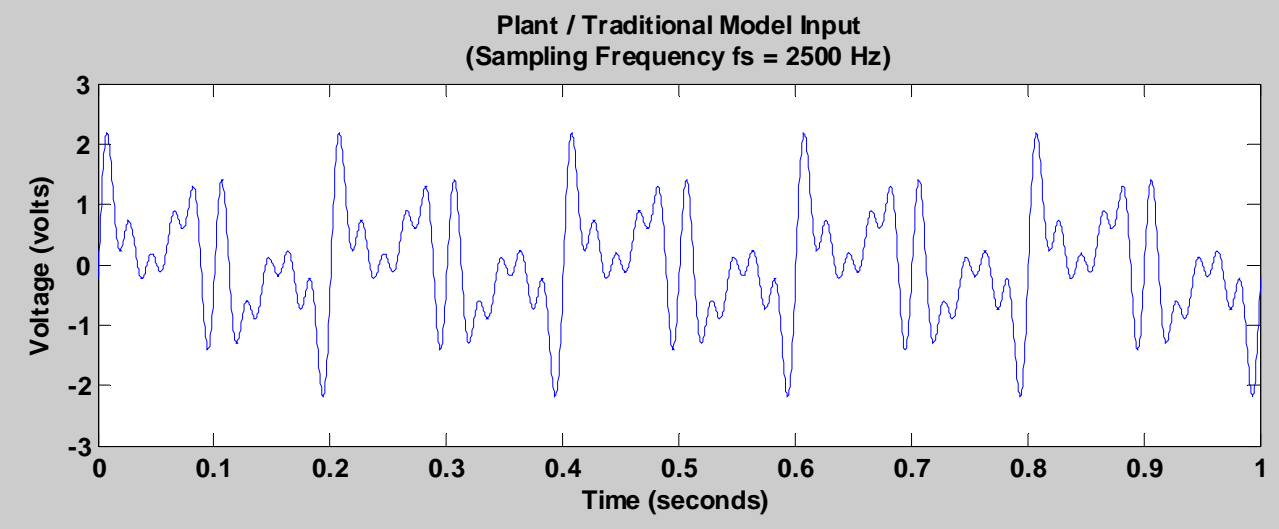

Traditional Model Output \& Plant Output (Target)

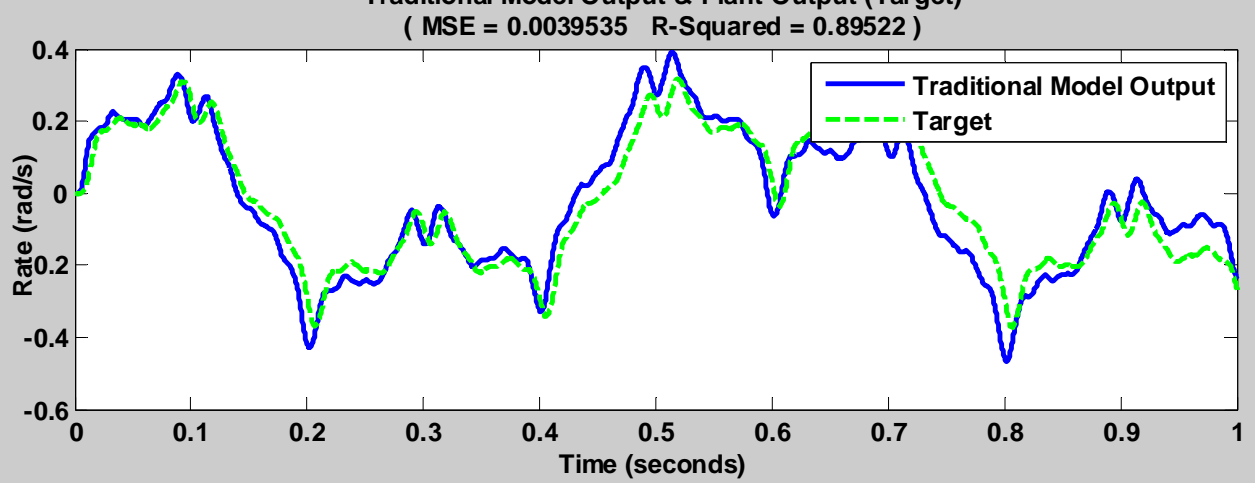

Figure 5.15: LTI Model Simulation Results (Multiple Frequency) 

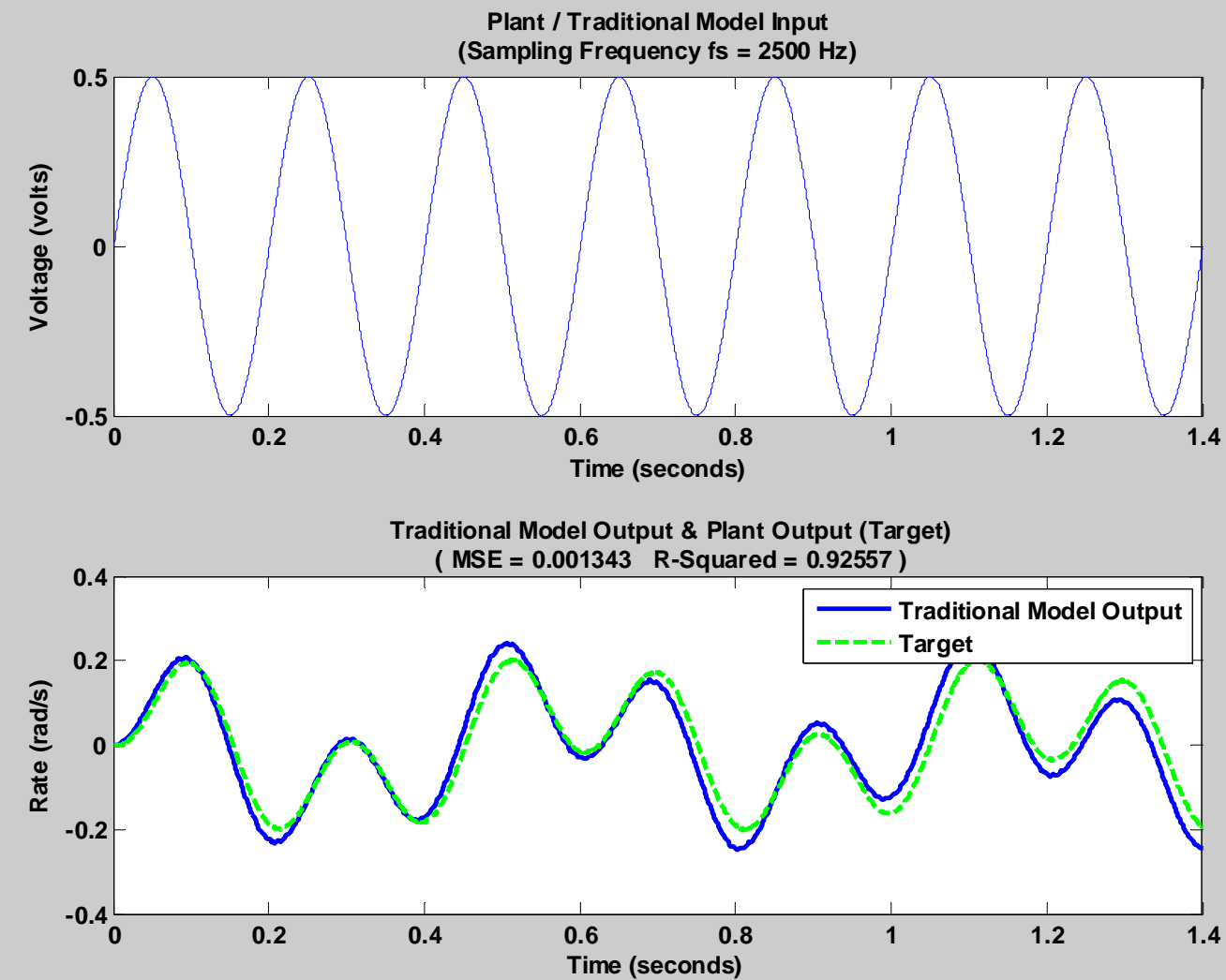

Figure 5.16: LTI Model Simulation Results (5 Hz Sine Wave) 


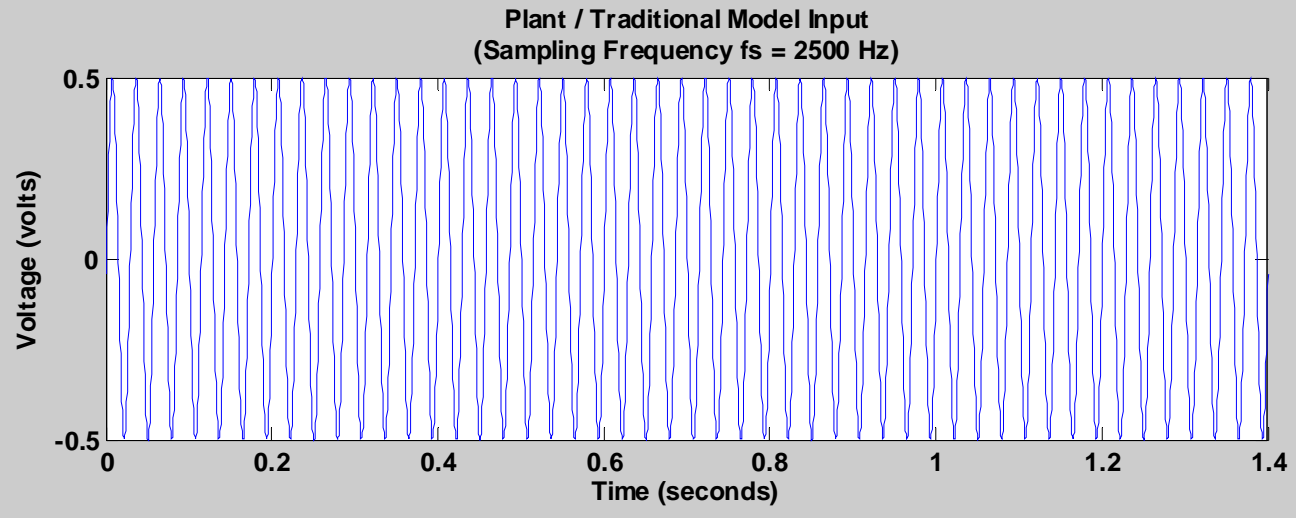

Traditional Model Output \& Plant Output (Target) ( MSE $=0.00020419$ R-Squared $=0.40264$ )

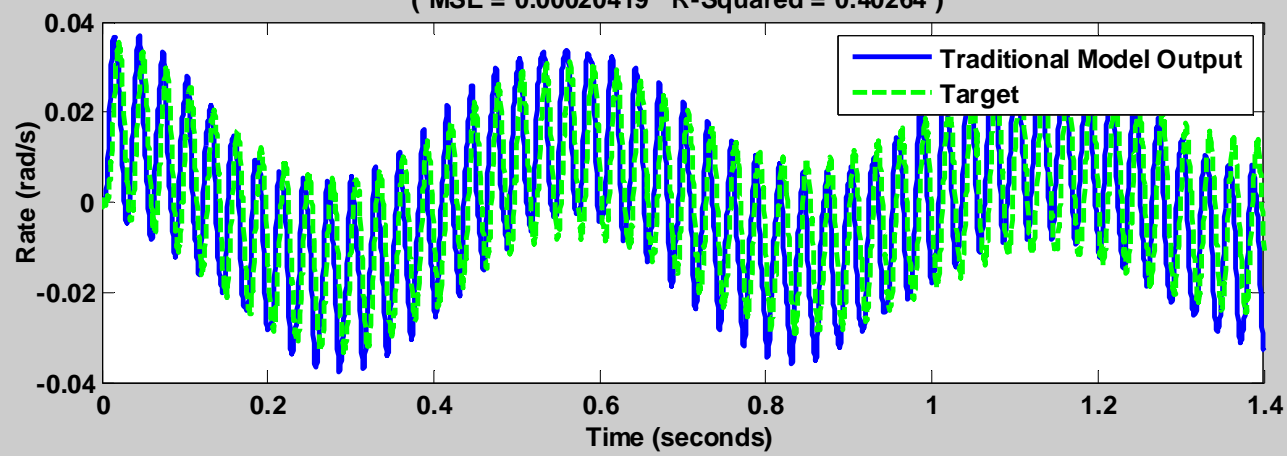

Figure 5.17: LTI Model Simulation Results (35 Hz Sine Wave) 


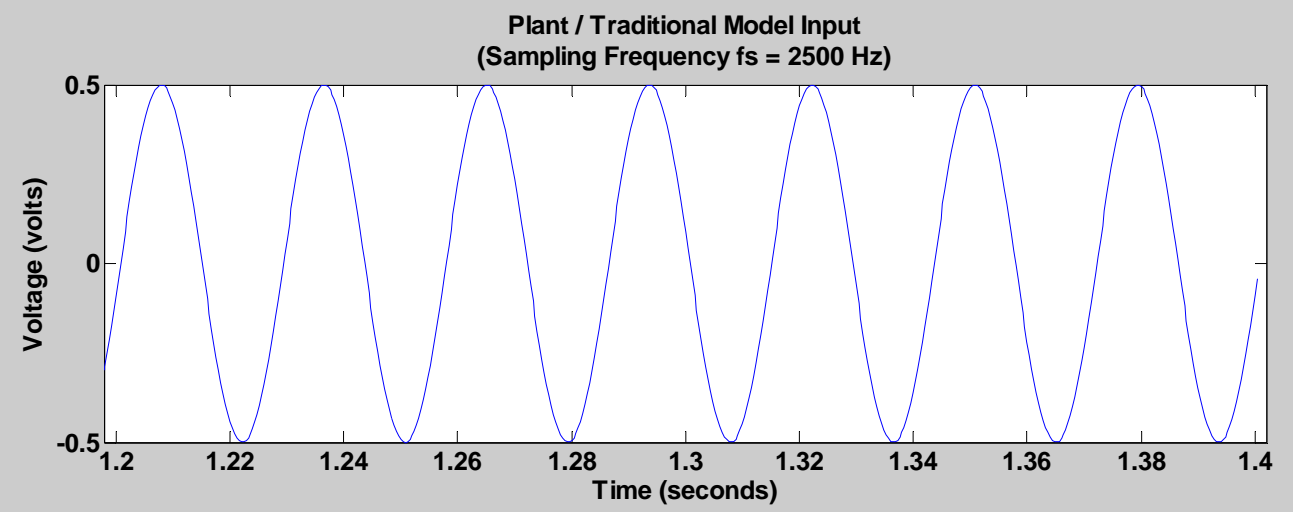

Traditional Model Output \& Plant Output (Target) ( MSE $=\mathbf{0 . 0 0 0 2 0 4 1 9}$ R-Squared $=0.40264)$

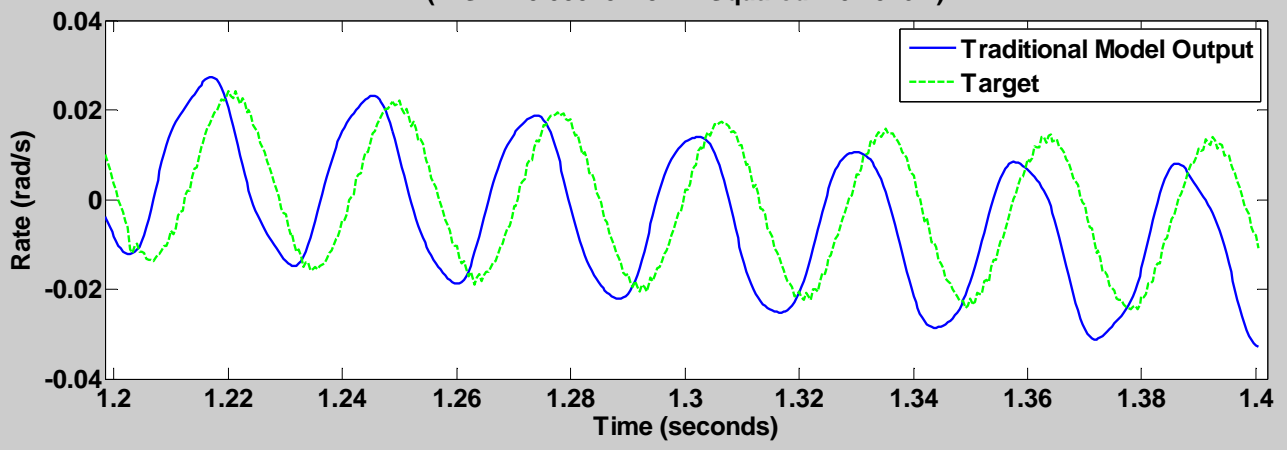

Figure 5.18: LTI Model Simulation Results ( $35 \mathrm{~Hz}$ Sine Wave) - Zoomed 


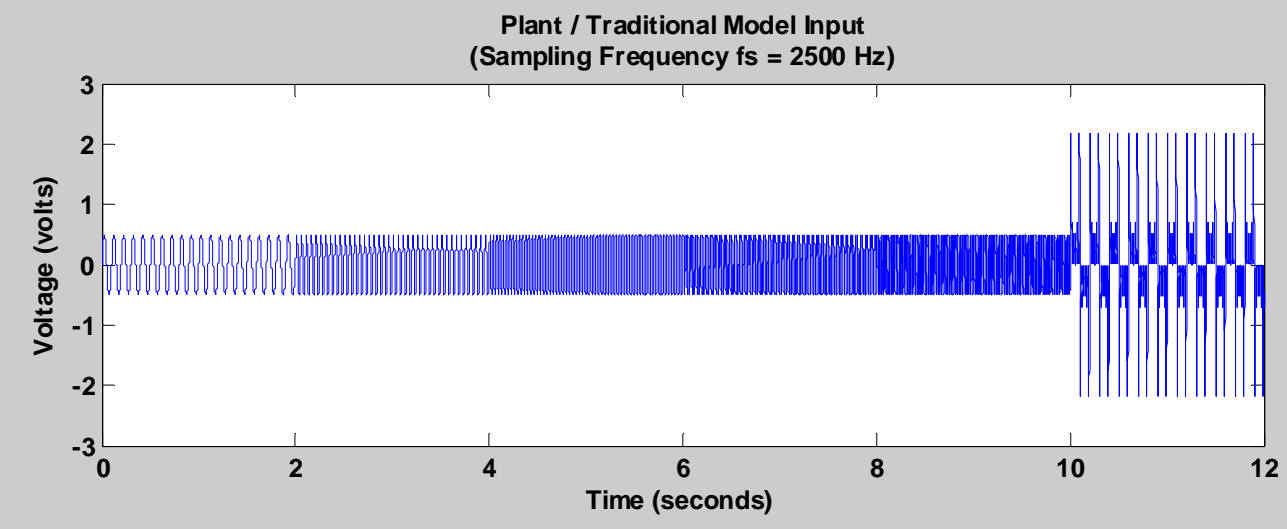

Traditional Model Output \& Plant Output (Target) ( MSE $=0.0015455$ R-Squared $=0.75175$ )

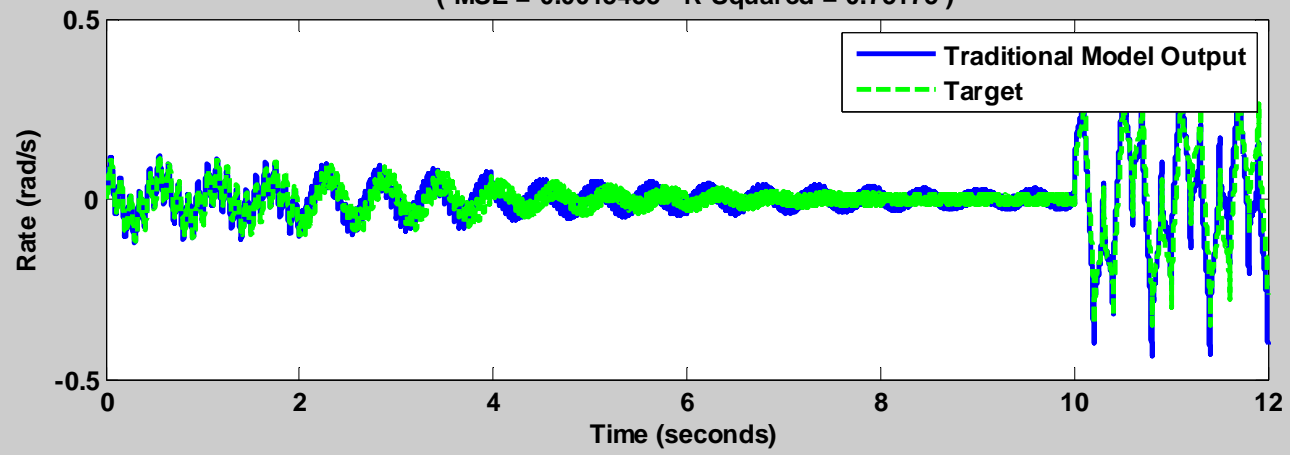

Figure 5.19: LTI Model Simulation Results (Single \& Multple Frequency) 
Plant / Traditional Model Input

(Sampling Frequency fs $=2500 \mathrm{~Hz}$ )

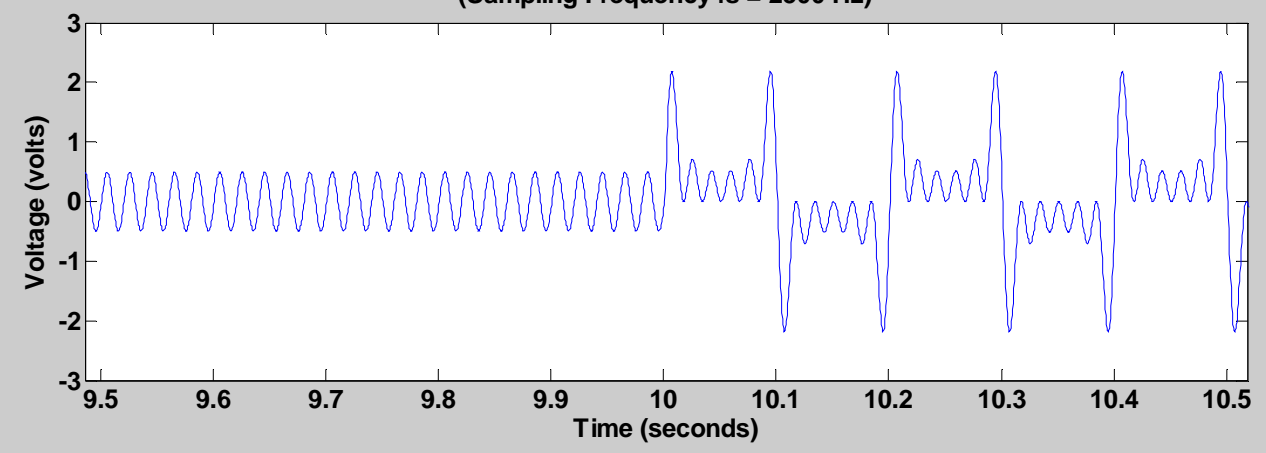

Traditional Model Output \& Plant Output (Target) ( MSE $=0.0015455$ R-Squared $=0.75175$ )

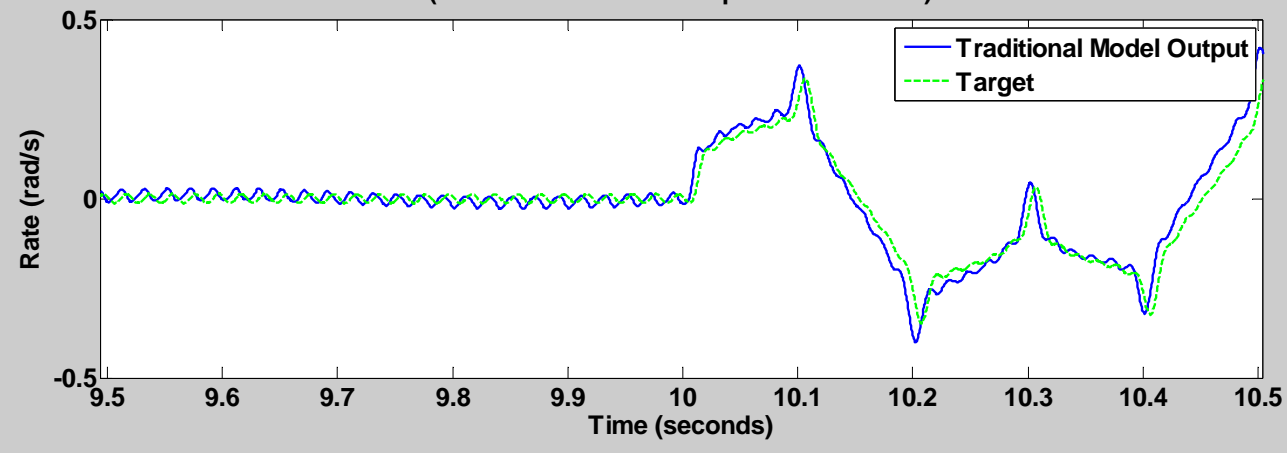

Figure 5.20: LTI Model Simulation Results (Single \& Multple Frequency) - Zoomed 

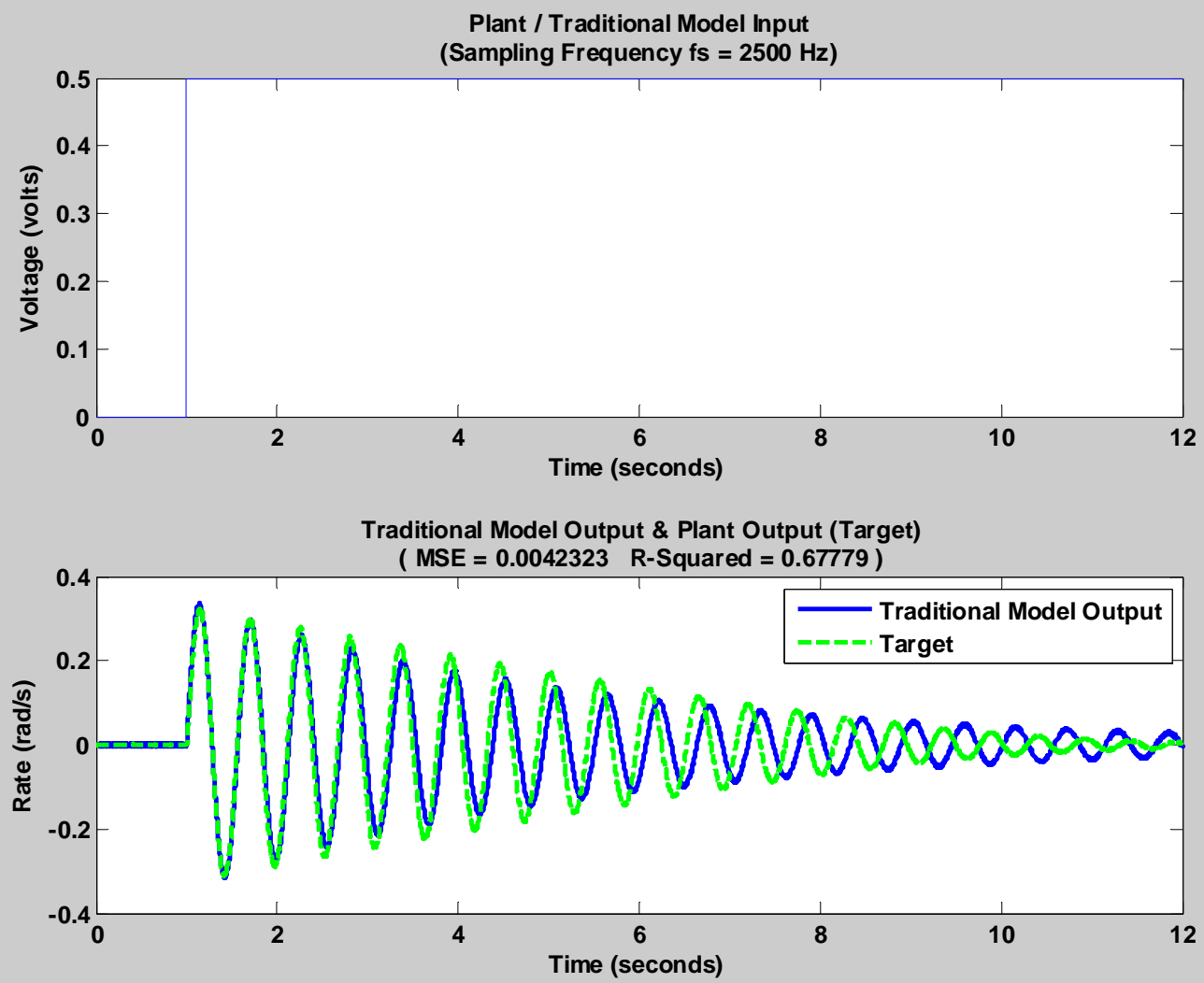

Figure 5.21: LTI Model Simulation Results (Step Function)

\section{4 - Traditional vs. Neural Network Model Results}

In summary, a traditional model was first created using the standard linear method for identification. The model's output was then simulated using the same input data sets used to simulate the neural network output in Section 4.4. After that, the traditional model output was compared to the real system output for each data set by calculating the mean squared error (MSE) and R-squared value. The neural network model output, traditional model output, and real system (target) output are plotted together for each training set and are shown below. In addition, Table 5.1 below shows the simulation 
results of both the traditional model and the neural network model (simulated in section $5.4)$.

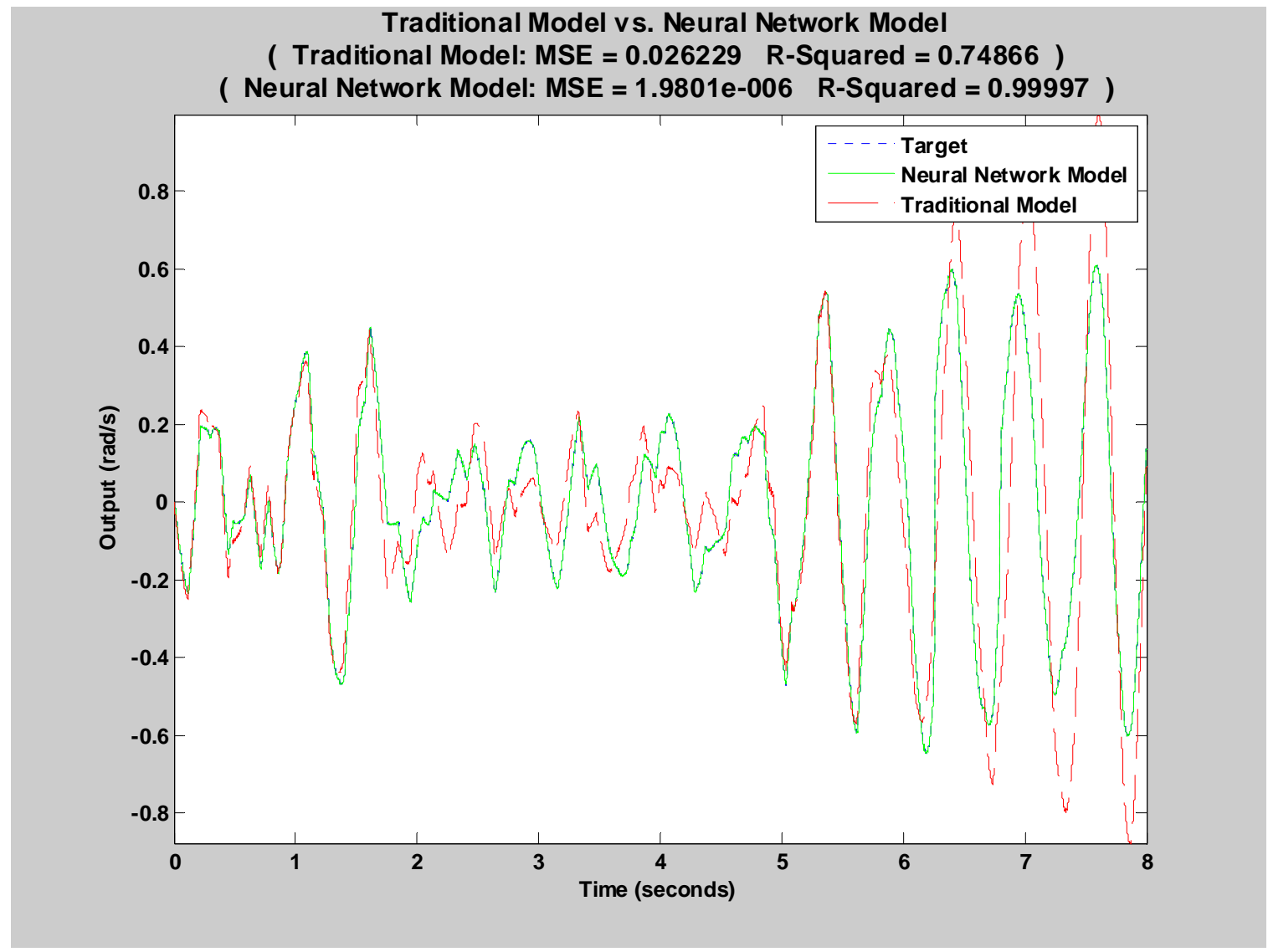

Figure 5.22: Traditional vs. NN Model Outputs: Random Input Signal 
Traditional Model vs. Neural Network Model

( Traditional Model: MSE $=6.1893 \mathrm{e}-005$ R-Squared $=0.85409$ )

( Neural Network Model: MSE $=1.9082 \mathrm{e}-006$ R-Squared $=0.9921$ )

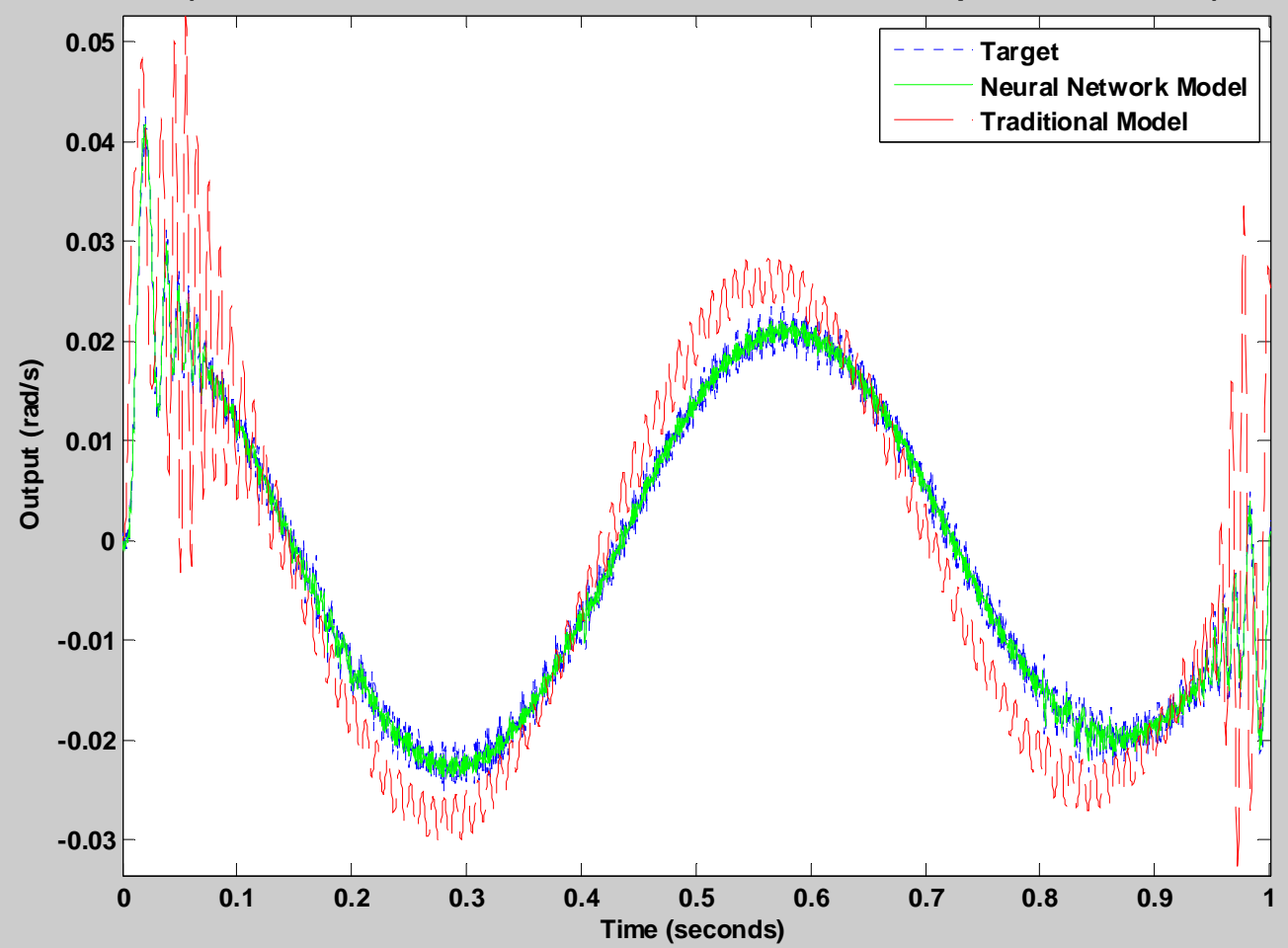

Figure 5.23: Traditional vs. NN Model Outputs: Chirp Input Signal 
Traditional Model vs. Neural Network Model

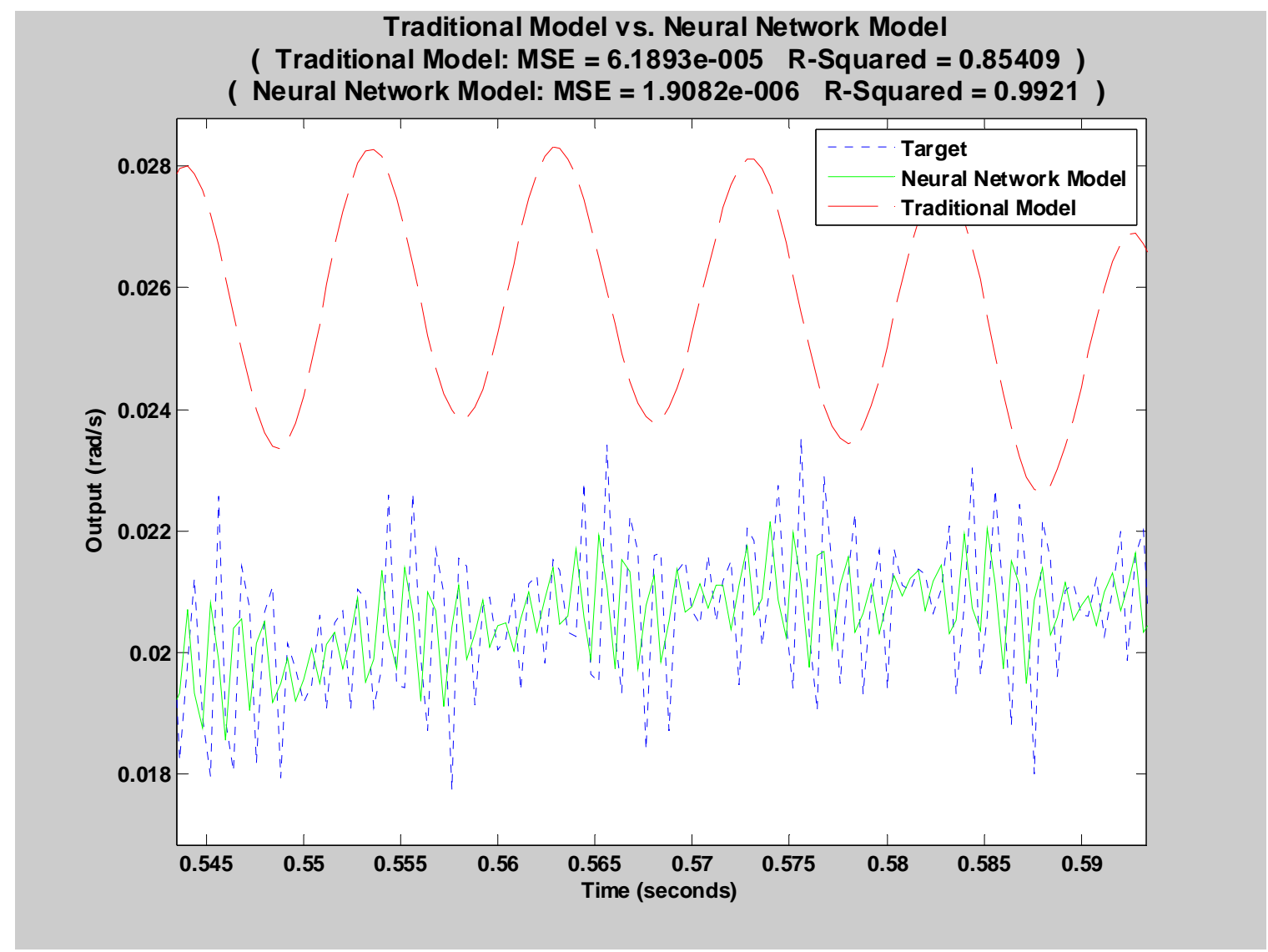

Figure 5.24: Traditional vs. NN Model Outputs: Chirp Input (Zoomed) 
Traditional Model vs. Neural Network Model

( Traditional Model: MSE $=\mathbf{0 . 0 0 3 9 5 3 5}$ R-Squared $=0.89522$ )

( Neural Network Model: MSE = 2.2514e-006 R-Squared =0.99993 )

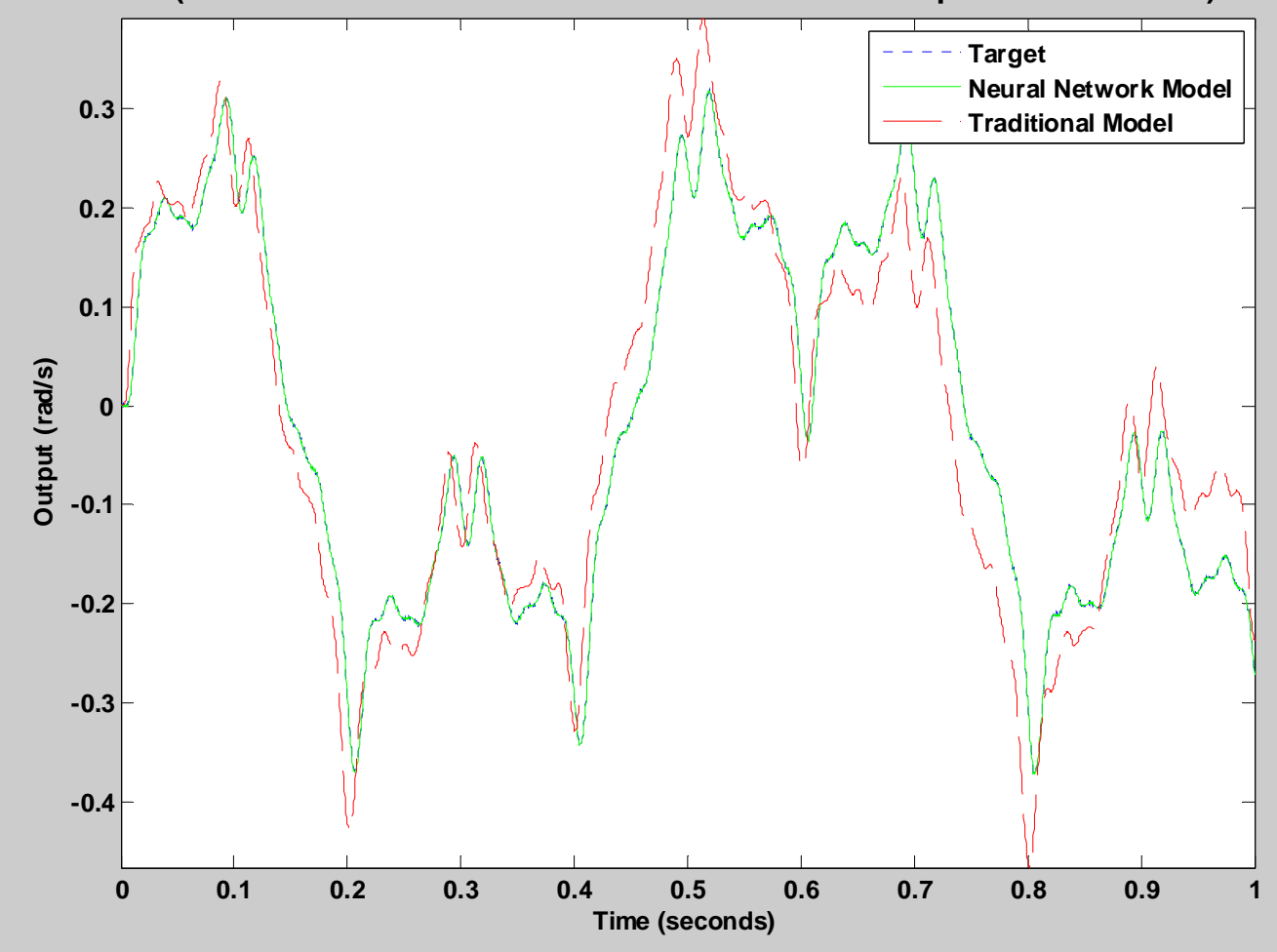

Figure 5.25: Traditional vs. NN Model Outputs: Multiple Freq Input Signal 
Traditional Model vs. Neural Network Model

( Traditional Model: MSE $=0.001343$ R-Squared $=0.92557$ )

( Neural Network Model: MSE $=1.7119 \mathrm{e}-006$ R-Squared $=0.99988$ )

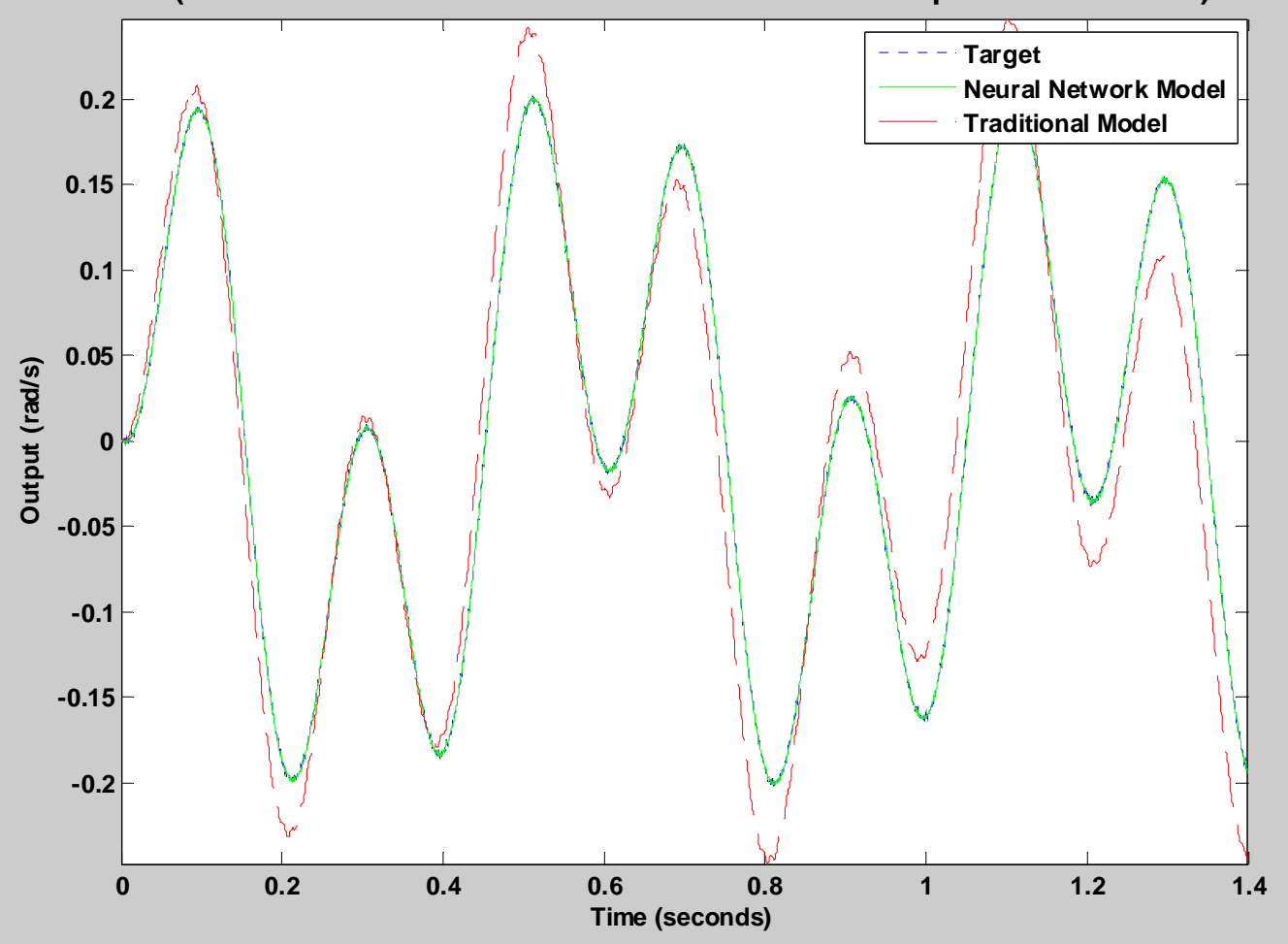

Figure 5.26: Traditional vs. NN Model Outputs: 5 Hz Sine Input Signal 
Traditional Model vs. Neural Network Model

( Traditional Model: MSE $=0.001343$ R-Squared $=0.92557$ )

( Neural Network Model: MSE $=1.7119 \mathrm{e}-006$ R-Squared $=0.99988$ )

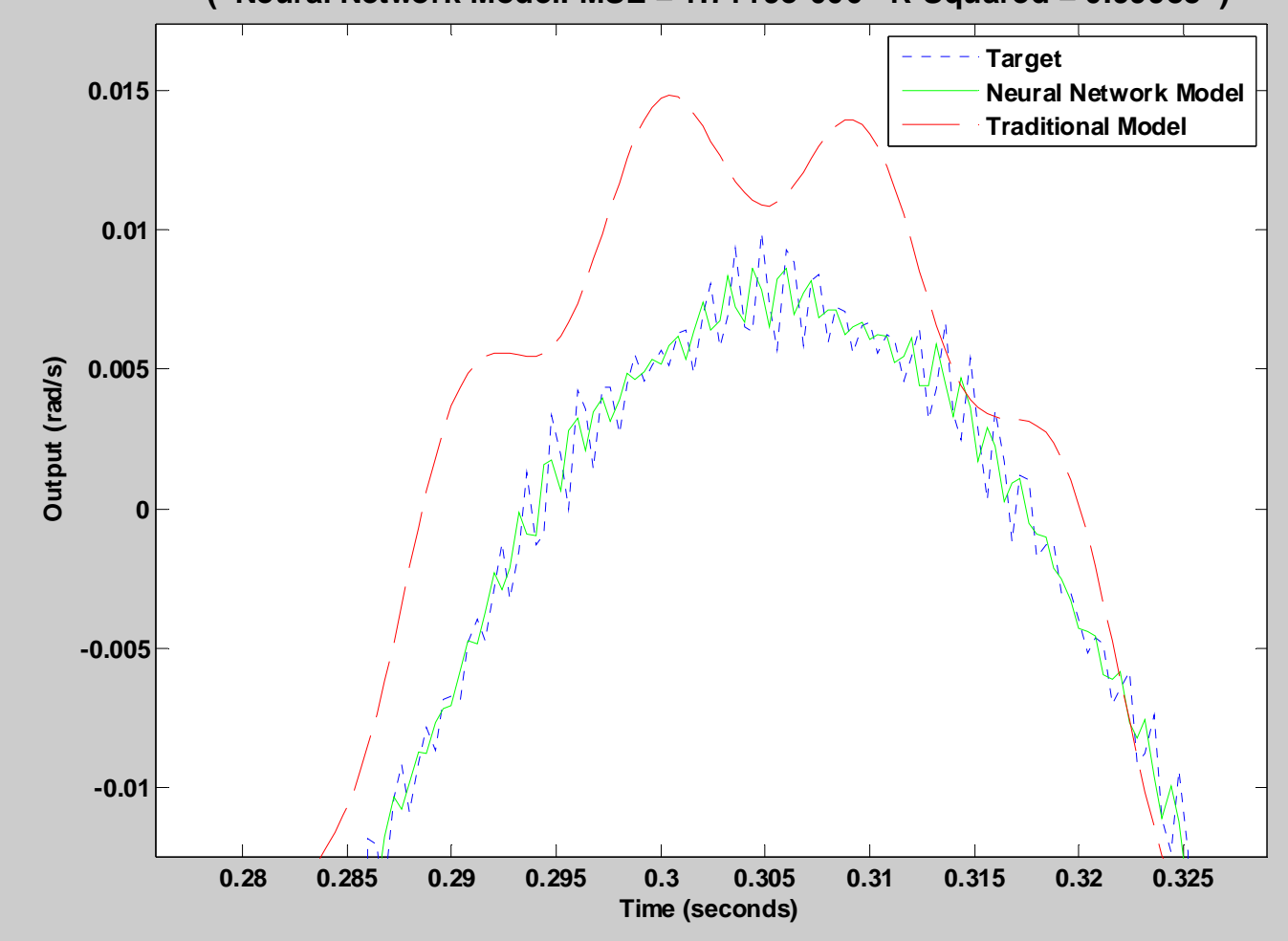

Figure 5.27: Traditional vs. NN Model Outputs: 5 Hz Sine Input (Zoomed) 
Traditional Model vs. Neural Network Model

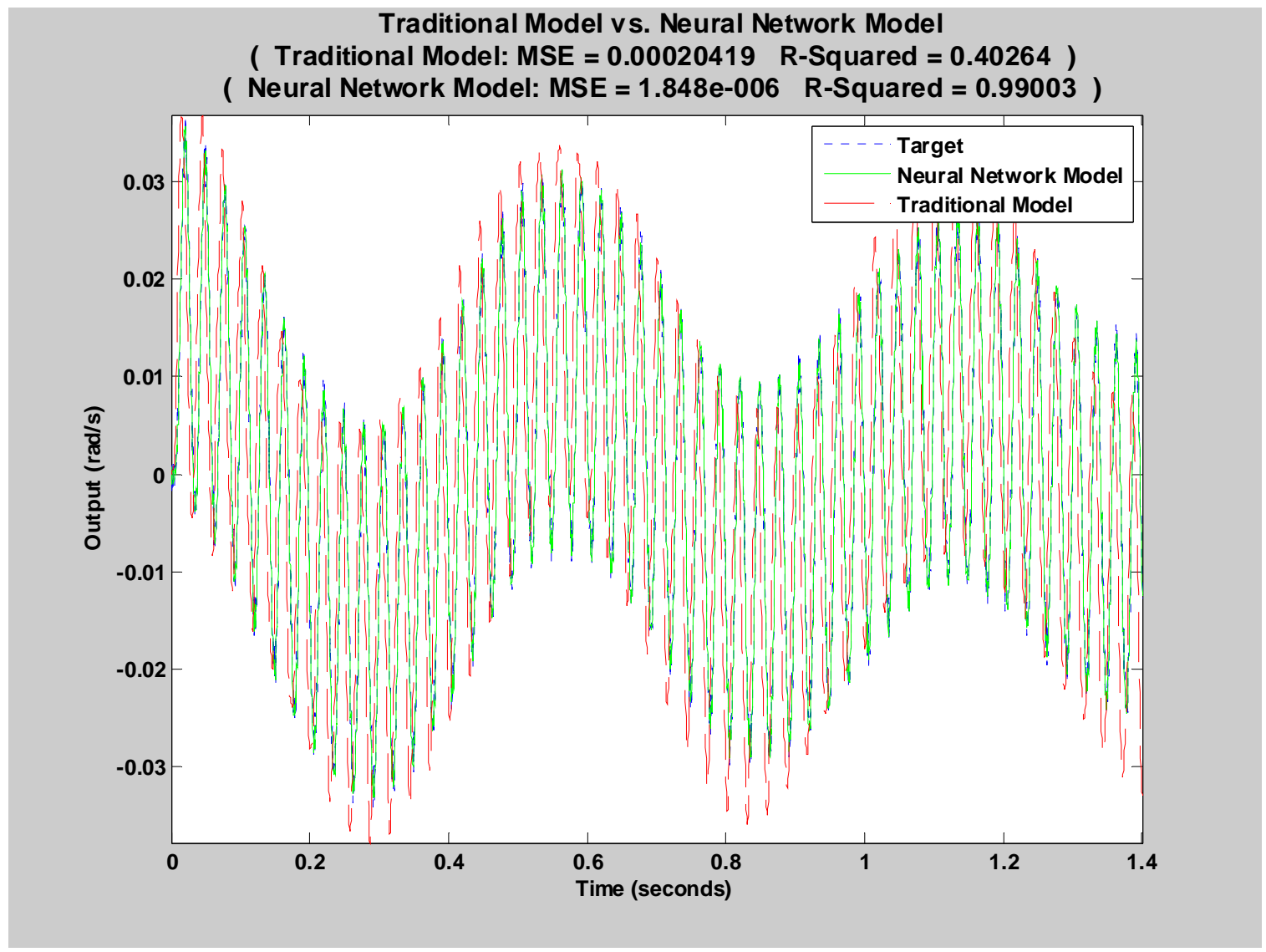

Figure 5.28: Traditional vs. NN Model Outputs: 35 Hz Sine Input Signal 
Traditional Model vs. Neural Network Model

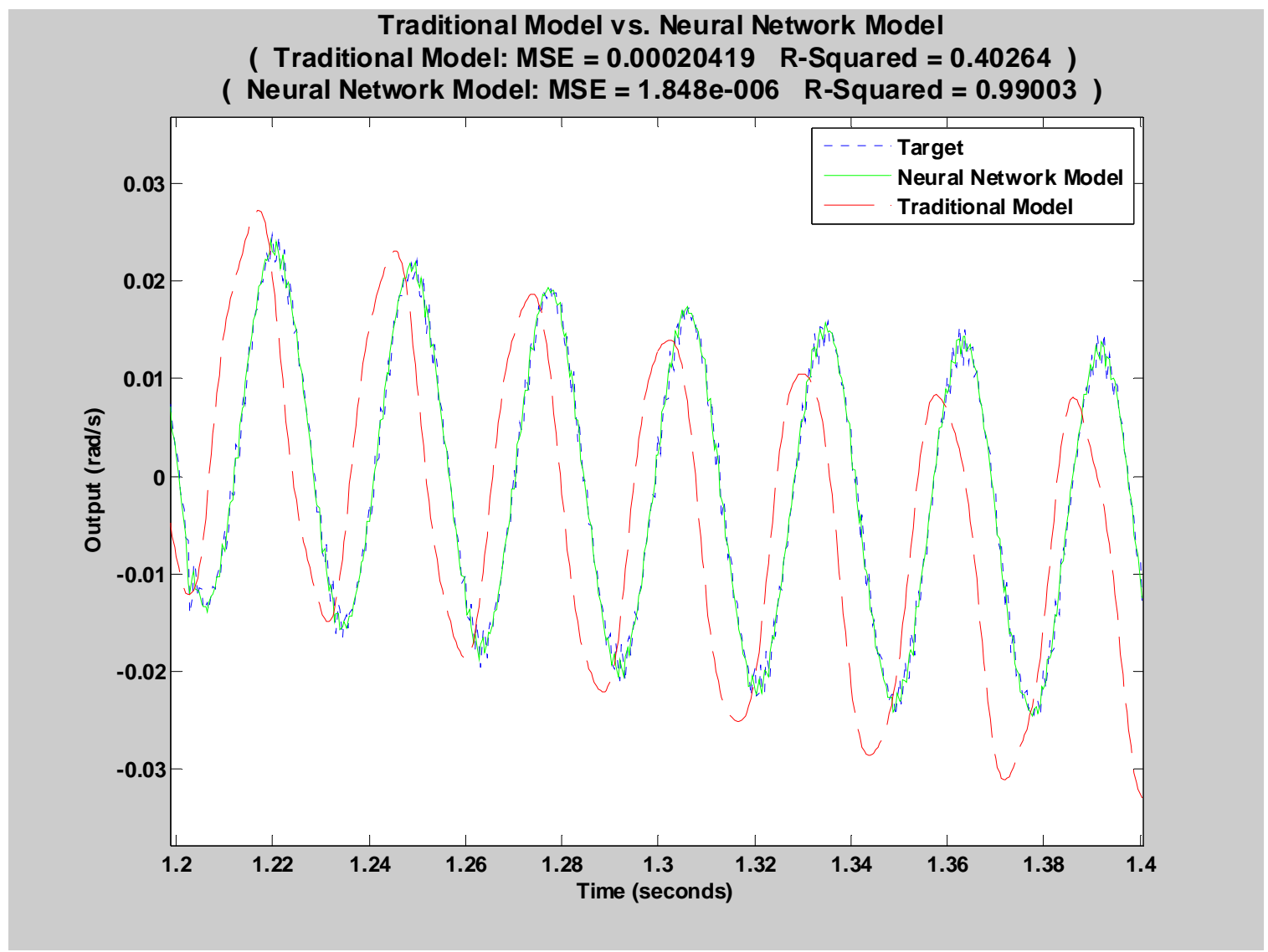

Figure 5.29: Traditional vs. NN Model Outputs: 35 Hz Sine (Zoomed 1) 
Traditional Model vs. Neural Network Model

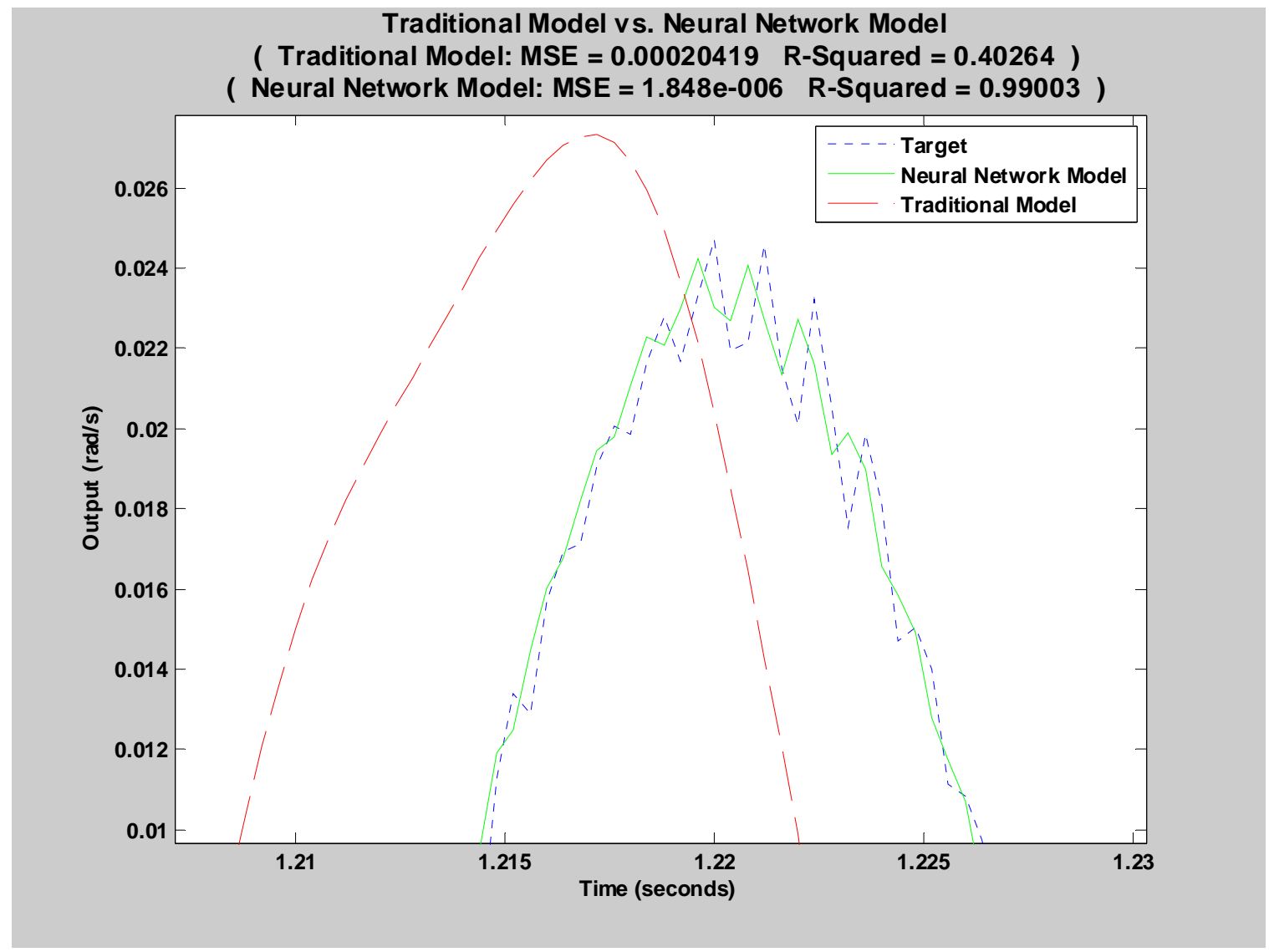

Figure 5.30: Traditional vs. NN Model Outputs: 35 Hz Sine (Zoomed 2) 
Traditional Model vs. Neural Network Model

( Traditional Model: MSE $=0.0015455$ R-Squared $=0.75175$ )

( Neural Network Model: MSE $=1.9481 \mathrm{e}-006$ R-Squared $=0.99963$ )

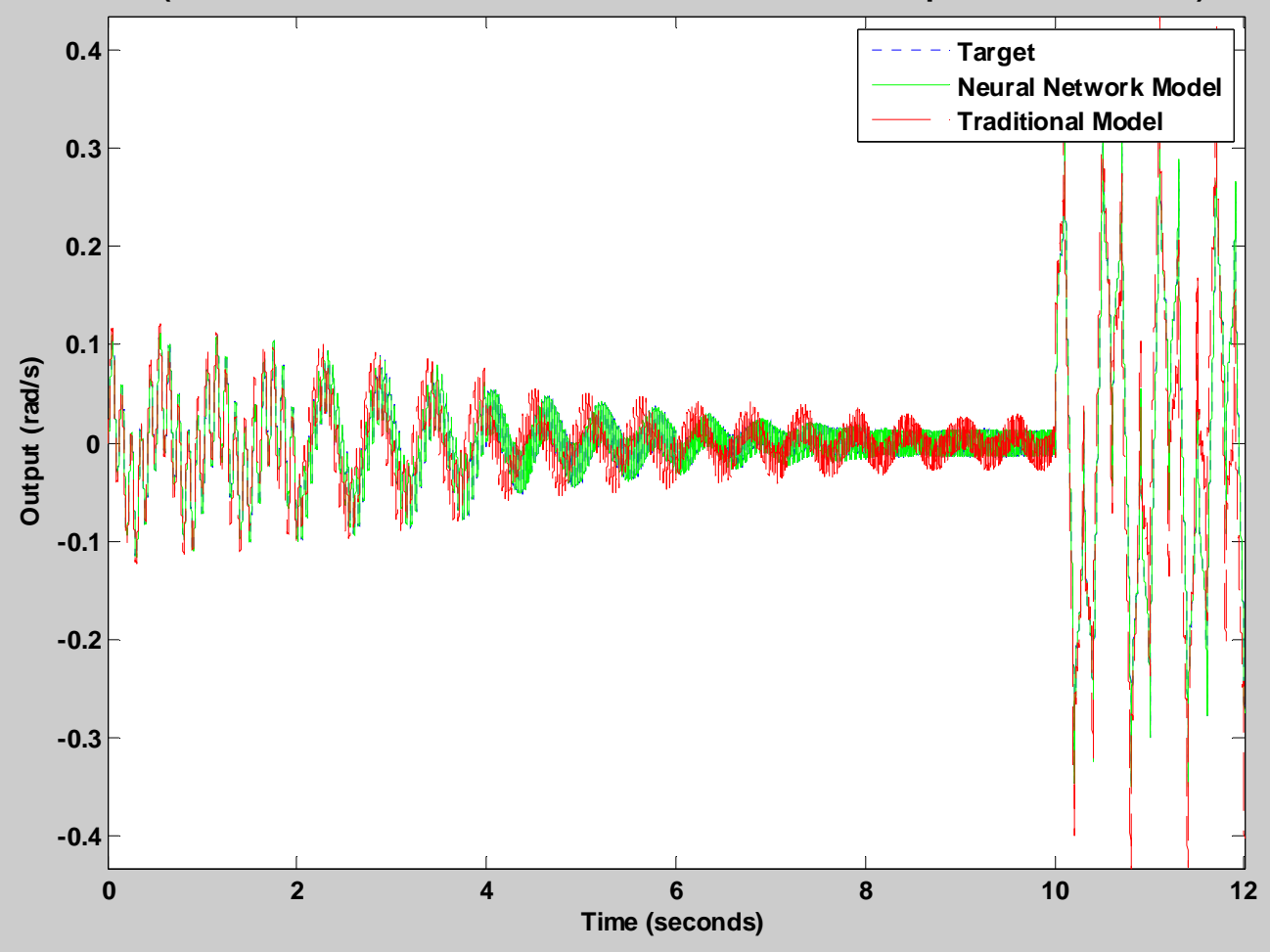

Figure 5.31: Traditional vs. NN Model Outputs: Single \& Multiple Freq Input Signal 
Traditional Model vs. Neural Network Model

( Traditional Model: MSE $=0.0015455$ R-Squared $=0.75175$ )

( Neural Network Model: MSE $=1.9481 \mathrm{e}-006$ R-Squared $=0.99963$ )

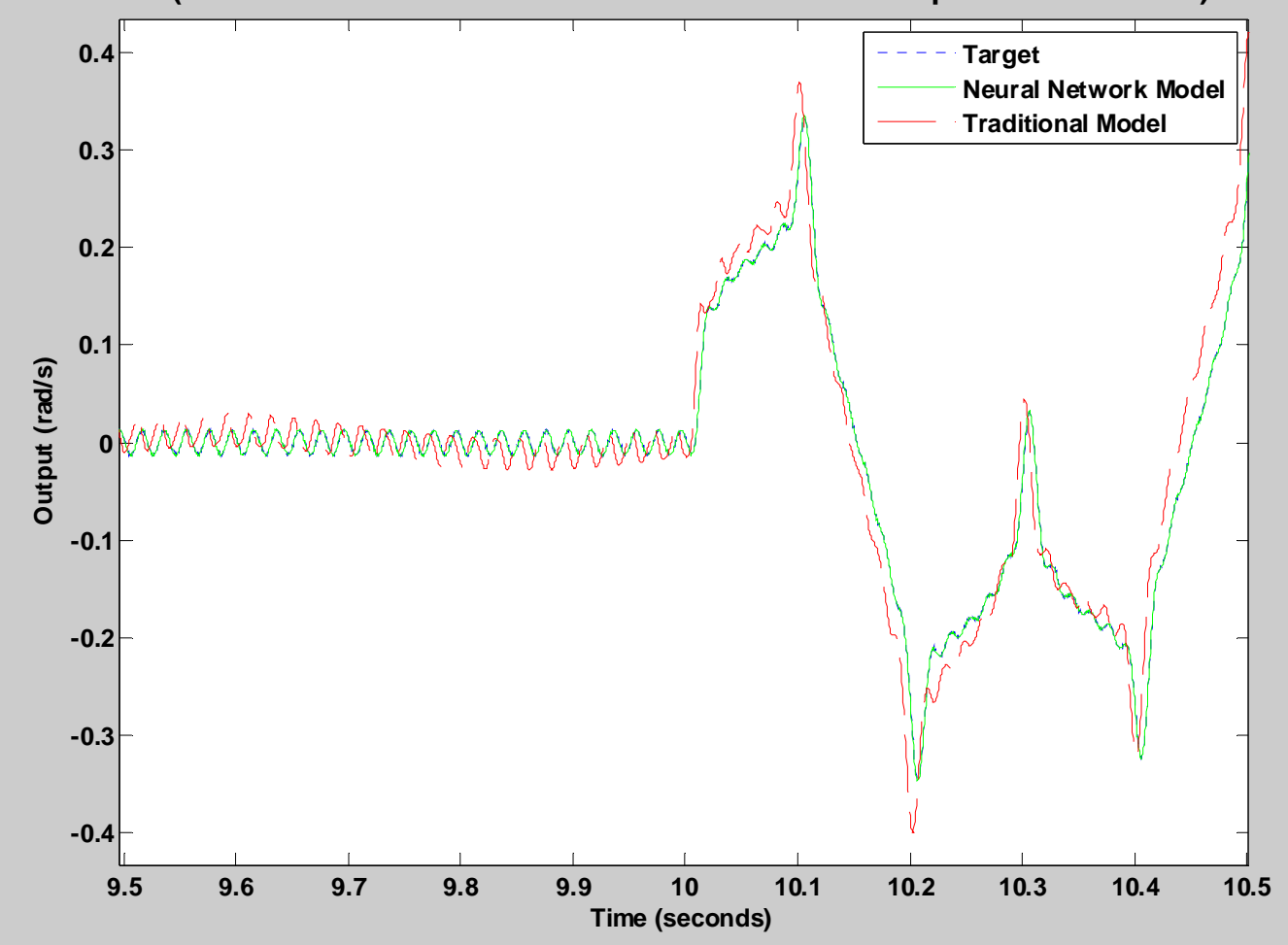

Figure 5.32: Traditional vs. NN Model Outputs: Single \& Mult. Freq (Zoomed) 
Traditional Model vs. Neural Network Model

( Traditional Model: MSE $=0.0042323$ R-Squared $=0.67779$ )

( Neural Network Model: MSE $=1.8851 \mathrm{e}-006$ R-Squared $=0.99986$ )

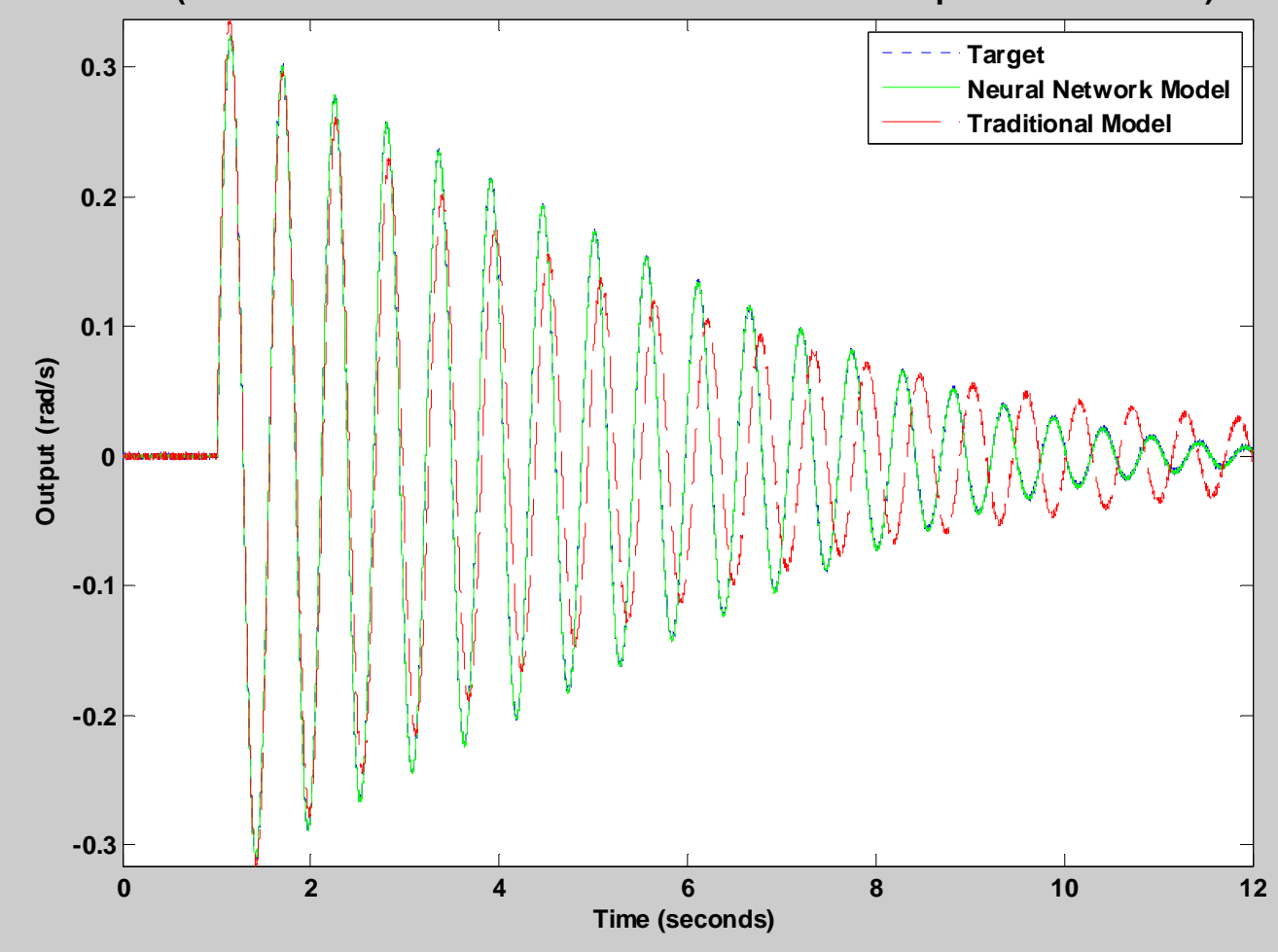

Figure 5.33: Traditional vs. NN Model Outputs: Step Function Input Signal 


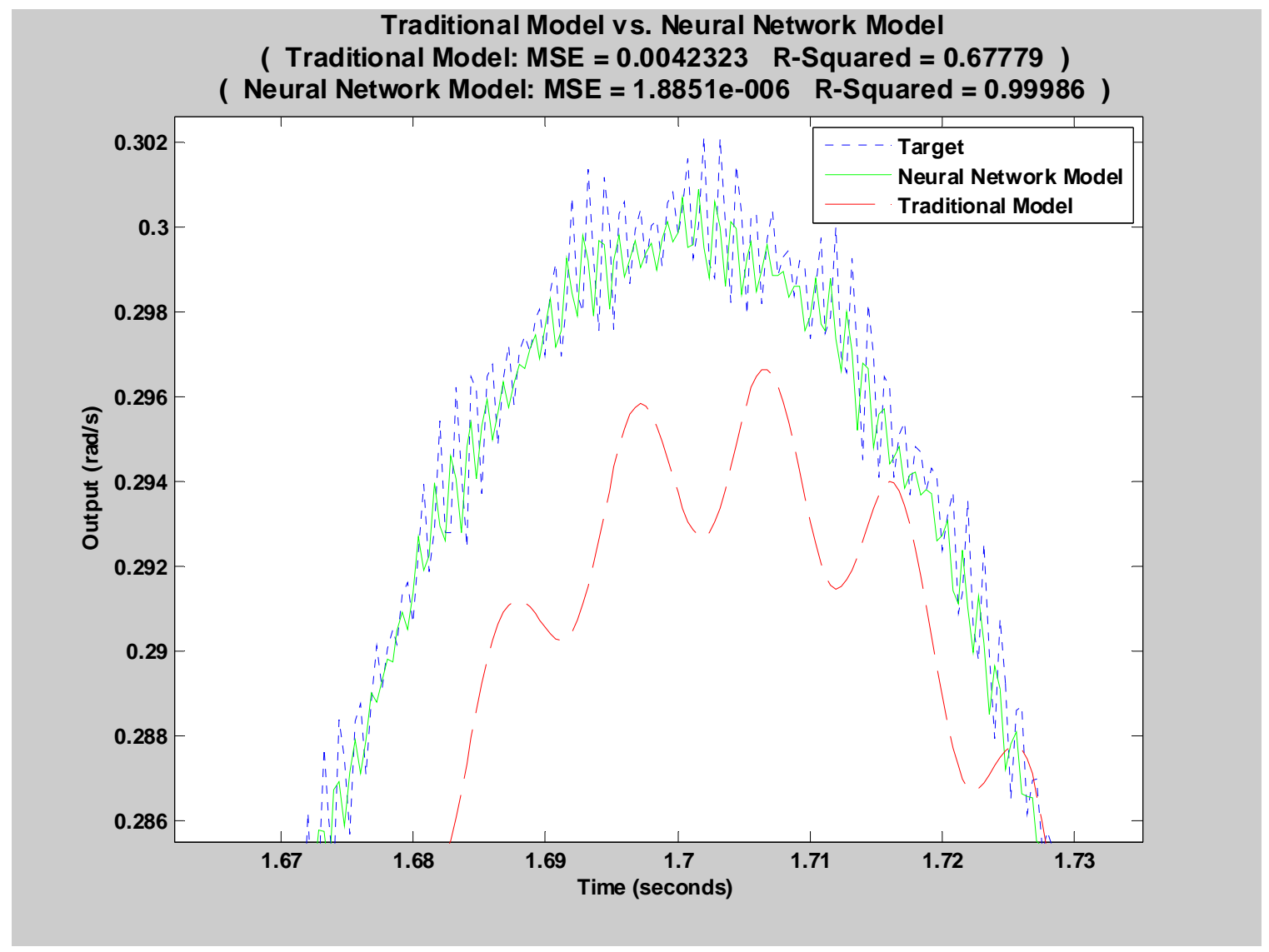

Figure 5.34:Traditional vs. NN Model Outputs: Step Function Input (Zoomed)

Table 5.1: Traditional vs. Neural Network Model Results

\begin{tabular}{|l|c|c|c|c|}
\hline \multicolumn{1}{|c|}{ Signal } & $\begin{array}{c}\text { Traditional } \\
\text { Model } \\
\text { MSE }\end{array}$ & $\begin{array}{c}\text { Neural } \\
\text { Network } \\
\text { MSE }\end{array}$ & $\begin{array}{c}\text { Traditional } \\
\text { Model } \\
\text { R-squared }\end{array}$ & $\begin{array}{c}\text { Neural } \\
\text { Network } \\
\text { R-squared }\end{array}$ \\
\hline Random Signal & 0.026229 & $1.9801 \mathrm{E}-6$ & 0.74866 & 0.99997 \\
\hline Chirp Signal & $6.1893 \mathrm{E}-5$ & $1.9082 \mathrm{E}-6$ & 0.85409 & 0.9921 \\
\hline Multiple Frequency Signal & 0.0039535 & $2.2514 \mathrm{E}-6$ & 0.89522 & 0.99993 \\
\hline 5 Hz Sine Wave & 0.001343 & $1.7119 \mathrm{E}-6$ & 0.92557 & 0.99988 \\
\hline 35 Hz Sine Wave & 0.00020419 & $1.848 \mathrm{E}-6$ & 0.40264 & 0.99003 \\
\hline Single \& Multiple Frequency & 0.0015455 & $1.948 \mathrm{E}-6$ & 0.75175 & 0.99963 \\
\hline Step Function & 0.0042323 & $1.8851 \mathrm{E}-6$ & 0.67779 & 0.99986 \\
\hline Average & $\mathbf{0 . 0 0 5 3 6 7 1}$ & $\mathbf{1 . 9 3 3 2 9 E - 6}$ & $\mathbf{0 . 7 5 0 8 2}$ & $\mathbf{0 . 9 9 7 3 4}$ \\
\hline
\end{tabular}

In conclusion, the neural network modeled the actual system more accurately than the traditional model. This can be seen by comparing the mean squared error and R- 
squared values between the two models. On average, the R-squared value of the neural network model was $32.8 \%$ better than the traditional model.

\section{Chapter 6: Conclusion and Future Works}

Gyro-stabilized cameras use gimbal systems for stabalizing cameras on different types of vehicles such as helicopters, boats or land vehicles. Typically, gimbal systems use traditional linear models to develop control stabilization strategies. However, linear models can lead to inaccuracies due to system complexities and non-linear relationships.

Aritificial neural networks, also referred to as "neural networks (NN)", are computer methodologies inspired by the biological systems of the human brain. Neural networks are non-linear information processing models that "learn" by classifying data and recognizing patterns. They can lead to a more accurate model of the gimbal system by mapping the input/output relationships of a system that includes characteristics left out of a traditional model.

Data gathered from experiments measuring the inertial characteristics of a gyrostabilized camera system were analyzed using the off-line batch training method to perform non-linear input/output mapping. A proprietary camera system was used for this study.

Computer simulation results demonstrate that neural networks can successfully model the inertial response of gyro-stabilized camera systems. Modeling of several types of time-domain data sets network structures, and sizes were analyzed. The network was tested on other data sets to find how well the network was able to generalize over all 
data sets. Larger network structures showed no apparent advantage over smaller structures and the network was able to generalize well over all measured data sets.

A MATLAB tool was developed and two other proprietary systems were modified to gather data from the gyro-stabilized camera system. Another MATLAB program was developed as a tool for training a neural network offline. The network was trained to perform input/output mapping of time history data, where the data represents the inertial response of the system. The Levenberg-Marquardt algorithm was used to train the network and the Nguyen-Widrow method was used to initialize the network weights. Motor voltage commands were used as inputs to the system and the inertial rate was used as the measured output.

Several different network structure types and sizes were compared. The networks were trained to map the input/output relationship of the same data set and were compared using the mean squared error, R-squared value and by observing plots of network output vs. target.

The network types included: (1) standard feedforward (FF), (2) focus time-delay (FTDNN), (3) nonlinear auto regressive network with exogenous inputs (NARX), and (4) a series parallel version of the nonlinear autoregressive network with exogenous inputs (NARXSP).

Only the NARXSP network structure was able to successfully map the input/target data set. The structure's performance was based on the mean squared error, R-squared value and visual observations. When comparing the different structure's abilities to map input/target data sets the structures from best to worst were: FF, FTDNN, NARX and NARXSP. 
Several NARXSP structure sizes were compared using the mean squared error and R-squared values. The different sizes included a 2 layer network with 5, 3 or 2 neurons with either a 5 or 2 tapped delay line on each input. No significant difference was found between the mean squared error and R-squared value for each of these network sizes.

The structure, NARXSP, was trained to map several input/target data sets with different input signals. The input signals included: (1) chirp signal, (2) step function, (3) random, (4) multiple frequency wave, (5) single frequencies with multiple frequency appended and (6) single frequency sine waves. The neural network was able to generalize well with the largest mean squared error of $2.25 \mathrm{E}-6$.

A traditional model was generated to compare with the neural network model. The model was tested utilizing the same data that tested the neural network in the generalization study. The neural network was a significant improvement over the traditional approach and generalized well with a best mean squared error (MSE) of 2,25E-6 and a minimum (worst) R-squared value of 0.99003 . By comparison, the traditional model's best MSE was 6.1893E-5 and best R-squared value was 0.92557.

The results of this work are indicative of the potential benefits for the future use of artificial neural network models in the study of online adaptive control of gyrostabilized camera systems. Certain neural network online control strategies, such as predictive control and model reference control, use neural network models and a gyrostabilized camera system can use these online control strategies if it uses a neural network model. The Levenberg-Marquardt algorithm could be used in an online adaptive control scheme. 


\section{Bibliography}

1. Haykin, Simon. Neural Networks A Comprehensive Foundation. New Jersey: Prentice-Hall Inc., 1999.

2. Ng., G.W. Application of Neural Networks to Adaptive Control of Nonlinear Systems. New York: John Wiley \& Sons Inc., 1997.

3. Narendra, K.S. and Parthasarathy, K., "Identification and Control of Dynamical Systems Using Neural Networks," IEEE Transactions on Neural Networks, vol.1, no.1, pp.4-27, Mar 1990. [Online]. Available: http://ieeexplore.ieee.org. [Accessed August 19, 2010].

4. Norgaard, M., O. Ravn, N.K. Poulsen and L.K. Hansen. Neural Networks for Modeling and Control of Dynamic Systems. London: Springer, 2000.

5. Pavelka, A., and A. Prochazka. "Algorithms for Initialization of Neural Network Weights". in Sborník príspevku 12. rocníku konference MATLAB 2004, volume 2, pages 453-459, 2004. [Online]. Available: http://dsp.vscht.cz. [Accessed August 20, 2010].

6. Plett, G.L., "Adaptive Inverse Control of Linear and Nonlinear Systems Using Dynamic Neural networks," IEEE Transactions on Neural Networks, vol.14, no.2, pp. 360- 376, Mar 2003. [Online]. Available: http://ieeexplore.ieee.org. [Accessed August 19, 2010].

7. Howard, Demuth, Beale Mark, and Hagan Martin, Neural Network Toolbox 6 User's Guide. The Mathworks Inc., 1992-2010.

8. Wellstead, P.E., and M.B. Zarrop, Self-Tuning Systems Control and Signal Processing. England: Chichester West Sussex, 1991. 
9. Hilkert, Jim. "Precision Line-of-Sight Control Engineering System Configuration, Analysis \& Design,” in Axsys Techonologies Precision Line-of Sight Control Engineering Seminar, Grass Valley, CA, August 16-17, 2010.

10. Hilkert, J.M, and D. Hullender, "Adaptive Control System Techniques Applied to Inertial Stabilization Systems," Proceedings from SPIE Acquisition, Tracking, and Pointing IV, vol. 1304, pp. 190-206, 1990.

11. Li, B.; Hullender, D., "Self-Tuning Controller for Nonlinear Inertial Stabilization Systems," IEEE Transactions on Control Systems Technology, vol.6, no.3, pp.428-434, May 1998.

12. Steven, Rogers C, "Disturbance Accommodation Design of a Notch Filter," in Signal Processing, Sensor Fusion, and Target Recognition Conference No. 12, Orlando, FL, vol. 5096, pp. 631-634, April, 2003.

13. Shan-zhong, Liu, and Sun Long-he. "Research on Stabilizing and Tracking Control of Electro-Optical Tracking and Sighting Platform Based on Fuzzy Control,” in International Conference on Measuring Technology and Mechatronics Automation, 2010 Henan Province, China, Henan University of Science and Technology, 2010.

14. Qing, Zhang, Tan Zhenfan, and Liang Ying, "Gyro-stabilized System Based on Auto-Disturbance Rejection Controller" in ISECS International Colloquium on Computing, Communication, Control, and Management, 2008.

15. Dongxi, M., C. Yujun and F. Dapeng, "Design and Implementation of an Electrooptical Tracking Servo System Via Composite Nonlinear Control Approach," in 2010 International Conference on Intelligent Computation Technology and 
Automation (ICICTA), vol.1, pp.1139-1142, 11-12 May 2010. [Online].

Available: http://ieeexplore.ieee.org. [Accessed August 19, 2010].

16. McBride, R.T., and F.E. Cellier, "Object-Oriented Bond-Graph Modeling of a Gyroscopically Stabilized Camera Platform," in ICBGM $6^{\text {th }}$ SCS Intl. Conference on Bond Graph Modeling and Simulation, pp. 223-230, Orlando, Florida, 2003.

17. Olsson, H., K. J. Astrom, C. C. de Wit, M. Gafvert, and P. Lischinsky, "Friction Models and Friction Compensation," European Journal of Control, vol. 4, no. 3, pp. 176-195, 1998.

18. Agilent Technologies, (2010, Nov 14), “The Fundamentals of Modal Testing: Application Note 243-3", [Online]. Available: http://www.home.agilent.com/agilent/home.jspx?cc=US\&lc=eng.

19. E. Marsh and A. Slocum, "Precision machine design topic 10 vibration control step 1: modal analysis," Penn State University, 1994.

20. D. Chou, "Dahl Friction Modeling", unpublished, B.S. thesis, Dept. of Mechanical Engineering, M.I.T., Cambridge, MA, 2004.

21. M. T. Hagan and M. B. Menhaj, "Training feedforward networks with the Marquardt algorithm," IEEE Transactions on Neural Networks, vol. 5, pp. 989-993, 1994.

22. D. Nguyen and B. Widrow, "Improving the learning speed of 2-layer neural networks by choosing initial values of the adaptive weights," 1990 IJCNN International Joint Conference on Neural Networks, 1990, pp. 21-26 vol.3. 


\section{Appendix A: MATLAB Code}

\section{Signal Creation GUI:}

\section{File: GUI_Create_i.m}

Description: Brings up a GUI interface which allows for creation of eight different types of signals. The user can also plot the signals and save the signals to an excel file. This file uses the create_i.m file for signal calculations.

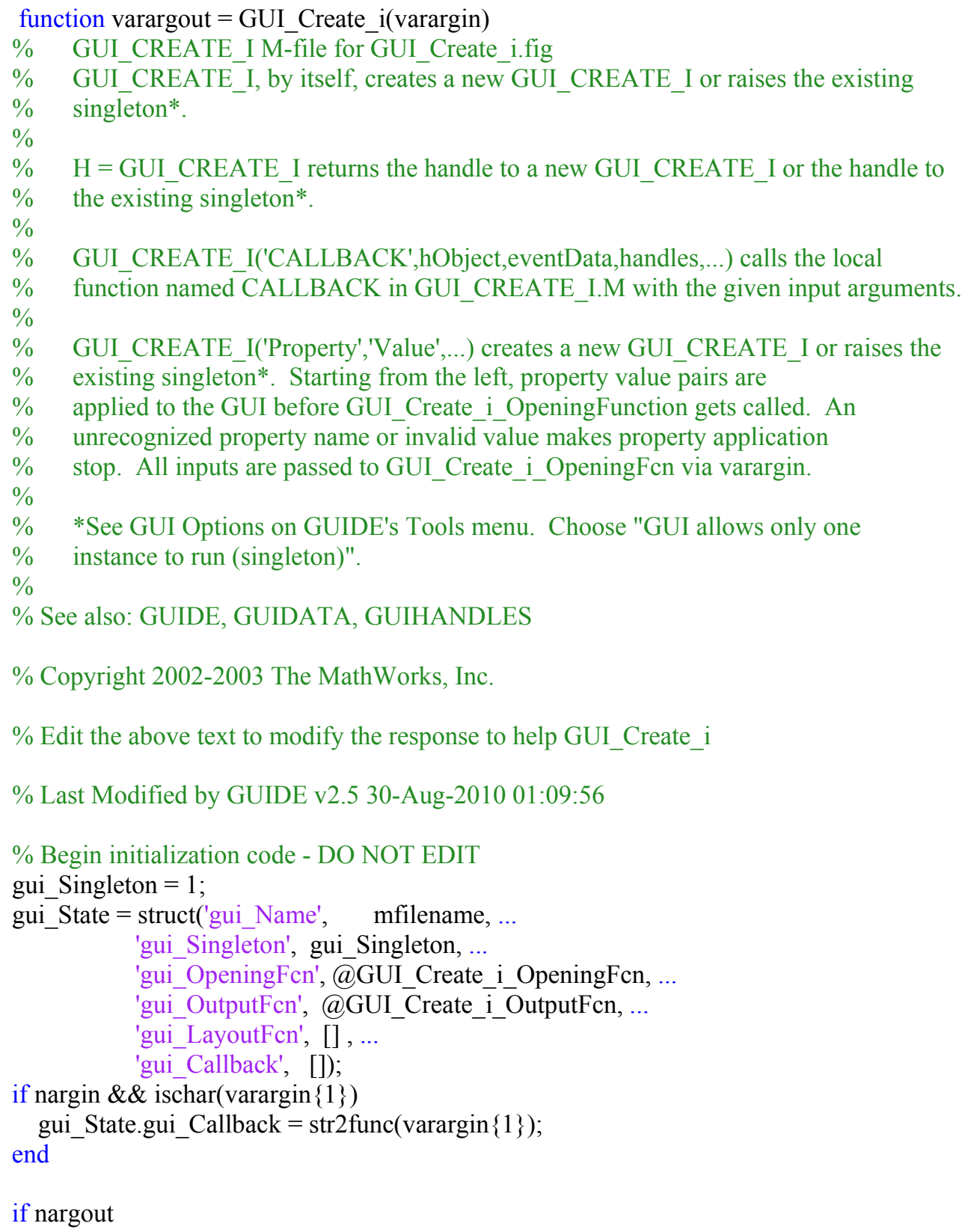




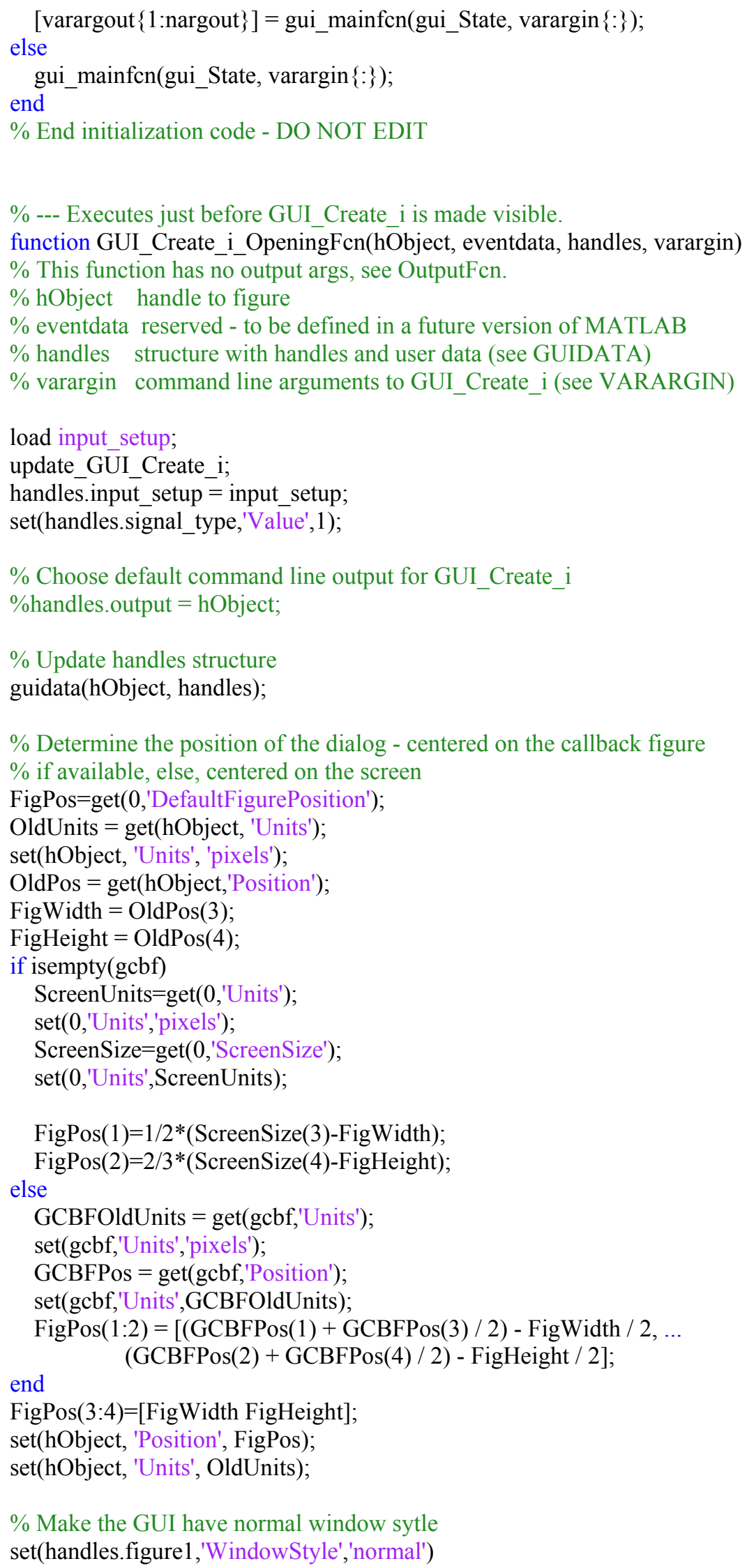




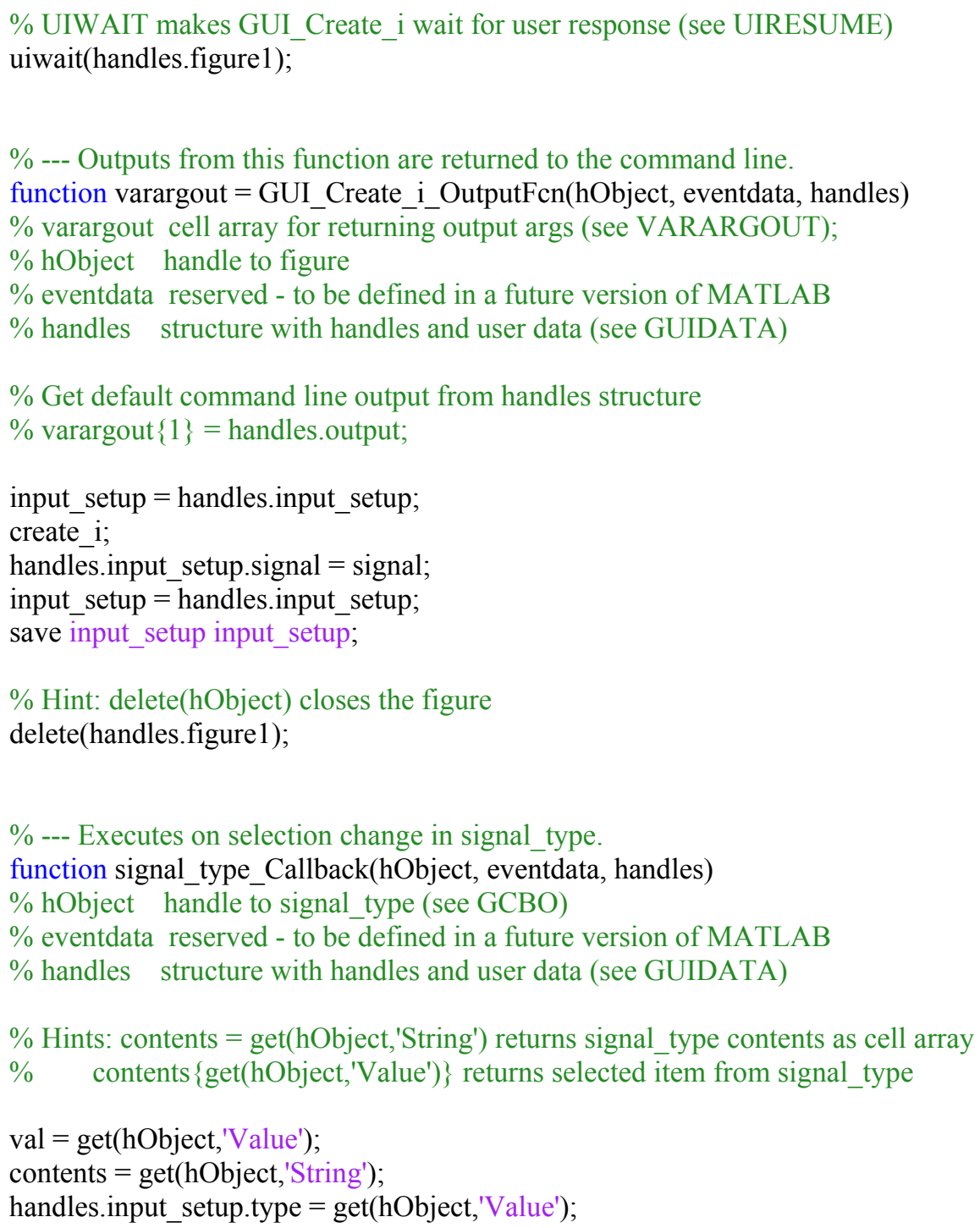


set(handles.uipanel6,'Visible','off');

set(handles.uipanel7,'Visible','off');

set(handles.uipanel9,'Visible','off');

set(handles.uipanelStepFunction,'Visible','off');

case 3

set(handles.panel_single_mult,'Visible','off');

set(handles.uipanel4,'Visible,',off');

set(handles.uipanel8,'Visible','off');

set(handles.uipanel5,'Visible','on');

set(handles.uipanel6,'Visible','off');

set(handles.uipanel7,'Visible','off');

set(handles.uipanel9,'Visible','off');

set(handles.uipanelStepFunction,'Visible','off');

case 4

set(handles.panel_single_mult,'Visible','off');

set(handles.uipanel4,'Visible','off');

set(handles.uipanel8,'Visible','off');

set(handles.uipanel5,'Visible','off');

set(handles.uipanel6,'Visible','on');

set(handles.uipanel7,'Visible','off');

set(handles.uipanel9,'Visible','off');

set(handles.uipanelStepFunction,'Visible','off');

case 5

set(handles.panel_single_mult,'Visible','off');

set(handles.uipanel4,'Visible','off');

set(handles.uipanel8,'Visible','off');

set(handles.uipanel5,'Visible','off');

set(handles.uipanel6, 'Visible','off');

set(handles.uipanel7,'Visible,',on');

set(handles.uipanel9,'Visible','off');

set(handles.uipanelStepFunction,'Visible','off');

case 6

set(handles.panel_single_mult,'Visible','off');

set(handles.uipanel4,'Visible','off');

set(handles.uipanel8,'Visible','on');

set(handles.uipanel5,'Visible','off');

set(handles.uipanel6,'Visible','off');

set(handles.uipanel7,'Visible','off');

set(handles.uipanel9,'Visible','off');

set(handles.uipanelStepFunction,'Visible','off');

case 7

set(handles.panel_single_mult,'Visible','off');

set(handles.uipanel4,'Visible','off');

set(handles.uipanel8,'Visible','off');

set(handles.uipanel5,'Visible','off');

set(handles.uipanel6,'Visible','off');

set(handles.uipanel7,'Visible','off');

set(handles.uipanel9,'Visible','on');

set(handles.uipanelStepFunction,'Visible','off');

case 8

set(handles.panel_single_mult,'Visible','off');

set(handles.uipanel4,'Visible','off');

set(handles.uipanel8,'Visible','off');

set(handles.uipanel5,'Visible','off');

set(handles.uipanel6,'Visible','off');

set(handles.uipanel7,'Visible','off'); 


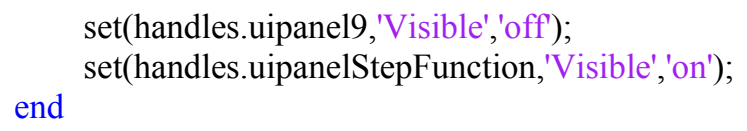

function num_samp_per_freq_type_1_Callback(hObject, eventdata, handles)

$\%$ hObject handle to num_samp_per_freq_type_1 (see GCBO)

$\%$ eventdata reserved - to be defined in a future version of MATLAB

$\%$ handles structure with handles and user data (see GUIDATA)

$\%$ Hints: get(hObject,'String') returns contents of num_samp_per_freq type_1 as text

$\% \quad$ str2double(get(hObject,'String')) returns contents of num_samp_per_freq_type_1 as a double 


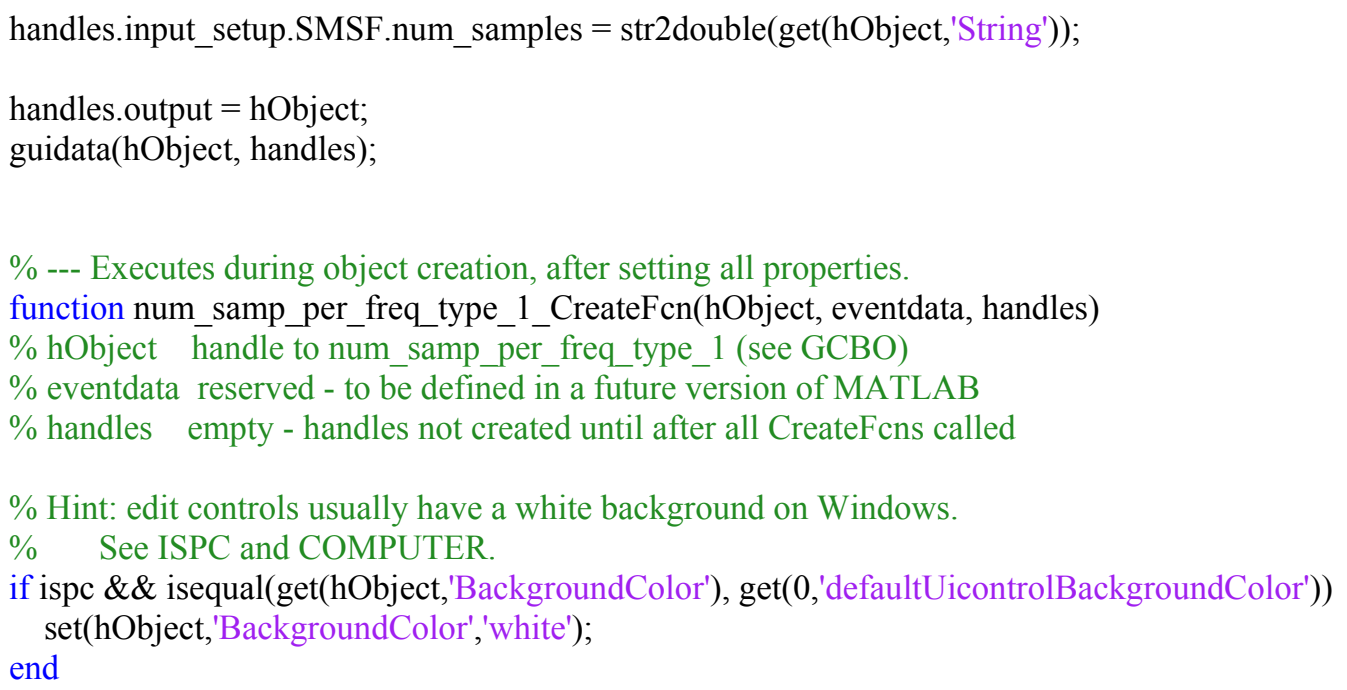

function freq_1_type_1_Callback(hObject, eventdata, handles)

$\%$ hObject handle to freq_1_type_1 (see GCBO)

$\%$ eventdata reserved - to be defined in a future version of MATLAB

$\%$ handles structure with handles and user data (see GUIDATA)

$\%$ Hints: get(hObject,'String') returns contents of freq_1_type_1 as text

$\% \quad$ str2double(get(hObject,'String')) returns contents of freq_1_type_1 as a double 


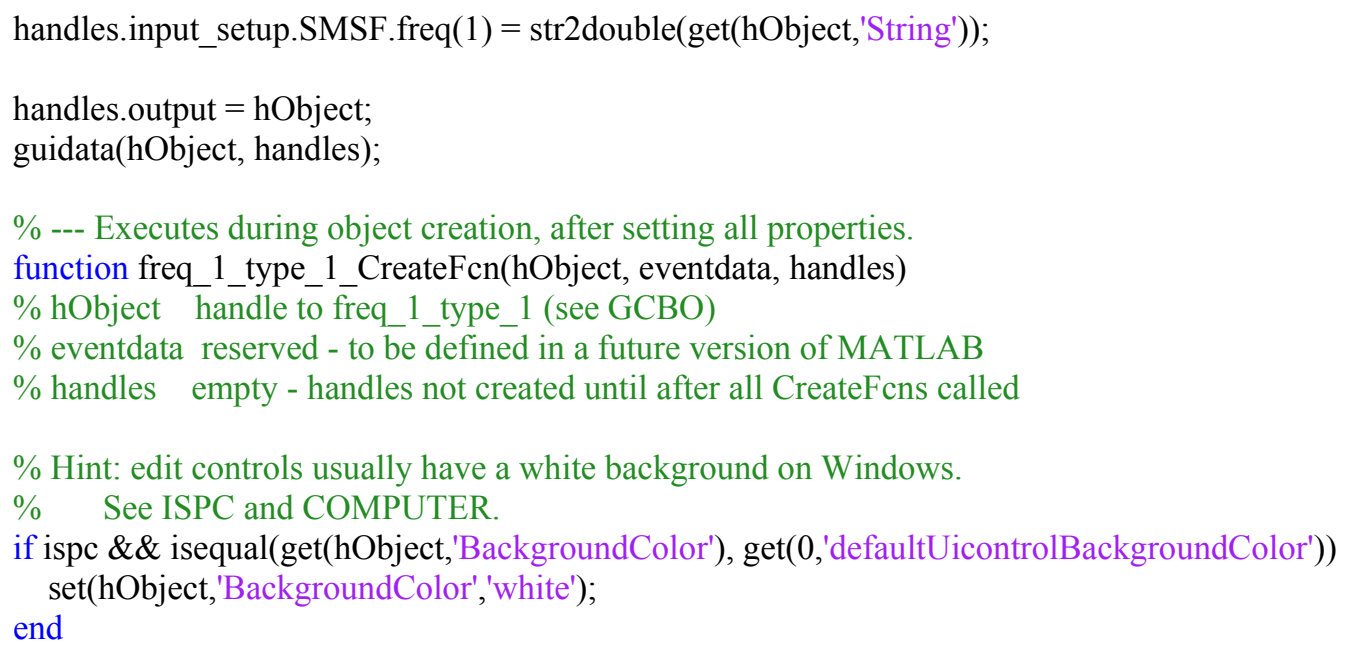


handles.output $=$ hObject;
guidata(hObject, handles);

$\%$--- Executes during object creation, after setting all properties.

function num_freq_type_1_CreateFcn(hObject, eventdata, handles)

$\%$ hObject handle to num_freq_type_1 (see GCBO)

$\%$ eventdata reserved - to be defined in a future version of MATLAB

$\%$ handles empty - handles not created until after all CreateFcns called

$\%$ Hint: popupmenu controls usually have a white background on Windows.

$\% \quad$ See ISPC and COMPUTER.

if ispc \&\& isequal(get(hObject,'BackgroundColor'), get( 0 ,'defaultUicontrolBackgroundColor')) set(hObject,'BackgroundColor','white'); end

function freq_3_type_1_Callback(hObject, eventdata, handles)

$\%$ hObject handle to freq 3 _type_1 (see GCBO)

$\%$ eventdata reserved - to be defined in a future version of MATLAB

$\%$ handles structure with handles and user data (see GUIDATA)

$\%$ Hints: get(hObject,'String') returns contents of freq_3_type_1 as text

$\% \quad$ str2double(get(hObject,'String')) returns contents of freq_3 type_1 as a double

handles.input_setup.SMSF.freq(3) = str2double(get(hObject,'String'));

handles.output $=$ hObject;

guidata(hObject, handles);

$\%$--- Executes during object creation, after setting all properties.

function freq_3_type_1_CreateFcn(hObject, eventdata, handles)

$\%$ hObject handle to freq 3 type_1 (see GCBO)

$\%$ eventdata reserved - to be defined in a future version of MATLAB

$\%$ handles empty - handles not created until after all CreateFcns called

$\%$ Hint: edit controls usually have a white background on Windows.

$\% \quad$ See ISPC and COMPUTER.

if ispc \&\& isequal(get(hObject,'BackgroundColor'), get( 0 ,'defaultUicontrolBackgroundColor')) set(hObject,'BackgroundColor','white'); end

function freq_4_type_1_Callback(hObject, eventdata, handles)

$\%$ hObject handle to freq 4 type 1 (see GCBO)

$\%$ eventdata reserved - to be defined in a future version of MATLAB

$\%$ handles structure with handles and user data (see GUIDATA)

$\%$ Hints: get(hObject,'String') returns contents of freq_4_type_1 as text

$\% \quad$ str2double(get(hObject,'String')) returns contents of freq_4_type_1 as a double

handles.input_setup.SMSF.freq(4) = str2double $($ get $($ hObject,'String'));

handles.output $=$ hObject;

guidata(hObject, handles); 


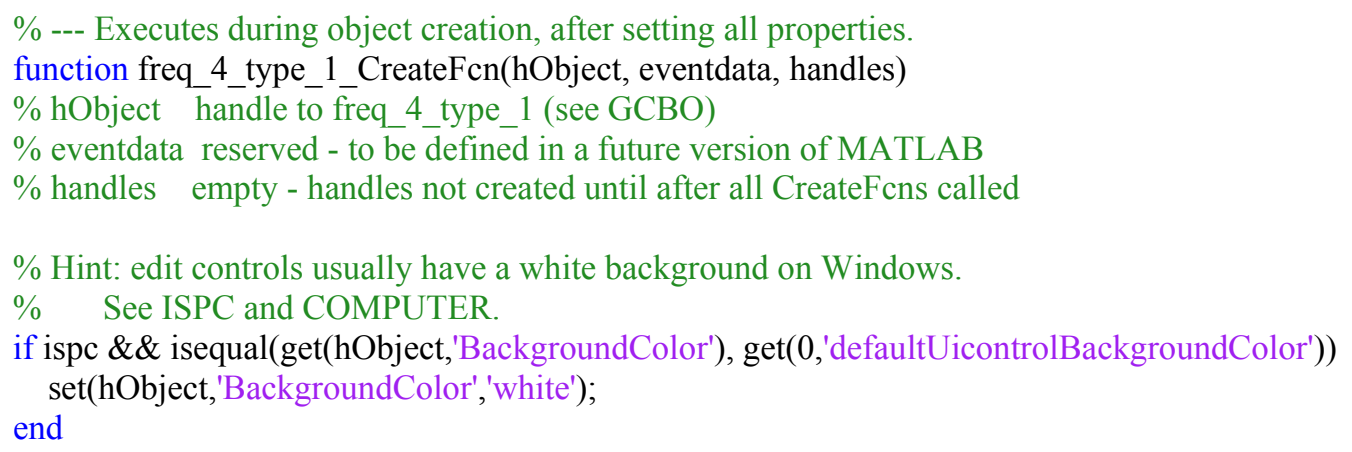

function freq_6_type_1_Callback(hObject, eventdata, handles)

$\%$ hObject handle to freq_6_type_1 (see GCBO)

$\%$ eventdata reserved - to be defined in a future version of MATLAB

$\%$ handles structure with handles and user data (see GUIDATA)

$\%$ Hints: get(hObject,'String') returns contents of freq_6_type_1 as text

$\% \quad$ str2double(get(hObject,'String')) returns contents of freq_6_type_1 as a double

handles.input_setup.SMSF.freq(6) = str2double(get(hObject,'String'));

handles.output $=$ hObject;

guidata(hObject, handles);

$\%$--- Executes during object creation, after setting all properties. 
function freq_6_type_1_CreateFcn(hObject, eventdata, handles)

$\%$ hObject handle to freq 6 type_1 (see GCBO)

$\%$ eventdata reserved - to be defined in a future version of MATLAB

$\%$ handles empty - handles not created until after all CreateFcns called

$\%$ Hint: edit controls usually have a white background on Windows.

$\% \quad$ See ISPC and COMPUTER.

if ispc \&\& isequal(get(hObject,'BackgroundColor'), get( 0 ,'defaultUicontrolBackgroundColor')) set(hObject,'BackgroundColor','white');

end

function samp_per_freq_type_2a_Callback(hObject, eventdata, handles)

$\%$ hObject handle to samp_per_freq_type_2a (see GCBO)

$\%$ eventdata reserved - to be defined in a future version of MATLAB

$\%$ handles structure with handles and user data (see GUIDATA)

\% Hints: get(hObject,'String') returns contents of samp_per_freq_type_2a as text

$\% \quad$ str2double(get(hObject,'String')) returns contents of samp_per_freq_type_2a as a double

handles.input_setup.SMSF_w_MFE.single_freq.num_samples = str2double(get(hObject,'String'));

handles.output $=$ hObject;

guidata(hObject, handles);

$\%$--- Executes during object creation, after setting all properties.

function samp_per_freq_type_2a_CreateFcn(hObject, eventdata, handles)

$\%$ hObject handle to samp_per_freq type_2a (see GCBO)

$\%$ eventdata reserved - to be defined in a future version of MATLAB

$\%$ handles empty - handles not created until after all CreateFcns called

$\%$ Hint: edit controls usually have a white background on Windows.

$\% \quad$ See ISPC and COMPUTER.

if ispc \&\& isequal(get(hObject,'BackgroundColor'), get( 0 ,'defaultUicontrolBackgroundColor')) set(hObject,'BackgroundColor','white');

end

function amplitude_type_2a_Callback(hObject, eventdata, handles)

$\%$ hObject handle to amplitude_type_2a (see GCBO)

$\%$ eventdata reserved - to be defined in a future version of MATLAB

$\%$ handles structure with handles and user data (see GUIDATA)

\% Hints: get(hObject,'String') returns contents of amplitude_type_2a as text

$\% \quad$ str2double(get(hObject,'String')) returns contents of amplitude_type_2a as a double

handles.input_setup.SMSF_w_MFE.single_freq.amplitude = str2double(get(hObject,'String'));

handles.output $=$ hObject;

guidata(hObject, handles);

$\%$--- Executes during object creation, after setting all properties.

function amplitude_type_2a_CreateFcn(hObject, eventdata, handles)

$\%$ hObject handle to amplitude_type_2a (see GCBO) 
$\%$ eventdata reserved - to be defined in a future version of MATLAB

$\%$ handles empty - handles not created until after all CreateFcns called

$\%$ Hint: edit controls usually have a white background on Windows.

$\% \quad$ See ISPC and COMPUTER.

if ispc \&\& isequal(get(hObject,'BackgroundColor'), get( 0 ,'defaultUicontrolBackgroundColor')) set(hObject,'BackgroundColor','white'); end

function freq_1_type_2a_Callback(hObject, eventdata, handles)

$\%$ hObject handle to freq_1_type_2a (see GCBO)

$\%$ eventdata reserved - to be defined in a future version of MATLAB

$\%$ handles structure with handles and user data (see GUIDATA)

$\%$ Hints: get(hObject,'String') returns contents of freq_1_type_2a as text

$\% \quad$ str2double(get(hObject,'String')) returns contents of freq_1 type 2a as a double

handles.input_setup.SMSF_w_MFE.single_freq.freq(1) = str2double(get(hObject,'String'));

handles.output $=$ hObject;

guidata(hObject, handles);

$\%$--- Executes during object creation, after setting all properties.

function freq_1_type_2a_CreateFcn(hObject, eventdata, handles)

$\%$ hObject handle to freq_1_type_2a (see GCBO)

$\%$ eventdata reserved - to be defined in a future version of MATLAB

$\%$ handles empty - handles not created until after all CreateFcns called

$\%$ Hint: edit controls usually have a white background on Windows.

$\% \quad$ See ISPC and COMPUTER.

if ispc \&\& isequal(get(hObject,'BackgroundColor'), get( 0 ,'defaultUicontrolBackgroundColor')) set(hObject,'BackgroundColor','white'); end

function freq_2_type_2a_Callback(hObject, eventdata, handles)

$\%$ hObject handle to freq 2 type 2 a (see GCBO)

$\%$ eventdata reserved - to be defined in a future version of MATLAB

$\%$ handles structure with handles and user data (see GUIDATA)

$\%$ Hints: get(hObject,'String') returns contents of freq_2_type_2a as text

$\% \quad$ str2double(get(hObject,'String')) returns contents of freq_2_type_2a as a double

handles.input_setup.SMSF_w_MFE.single_freq.freq(2) = str2double(get(hObject,'String'));

handles.output $=$ hObject;

guidata(hObject, handles);

$\%$--- Executes during object creation, after setting all properties.

function freq 2 type_2a_CreateFcn(hObject, eventdata, handles)

$\%$ hObject handle to freq_2 type_2a (see GCBO)

$\%$ eventdata reserved - to be defined in a future version of MATLAB

$\%$ handles empty - handles not created until after all CreateFcns called 


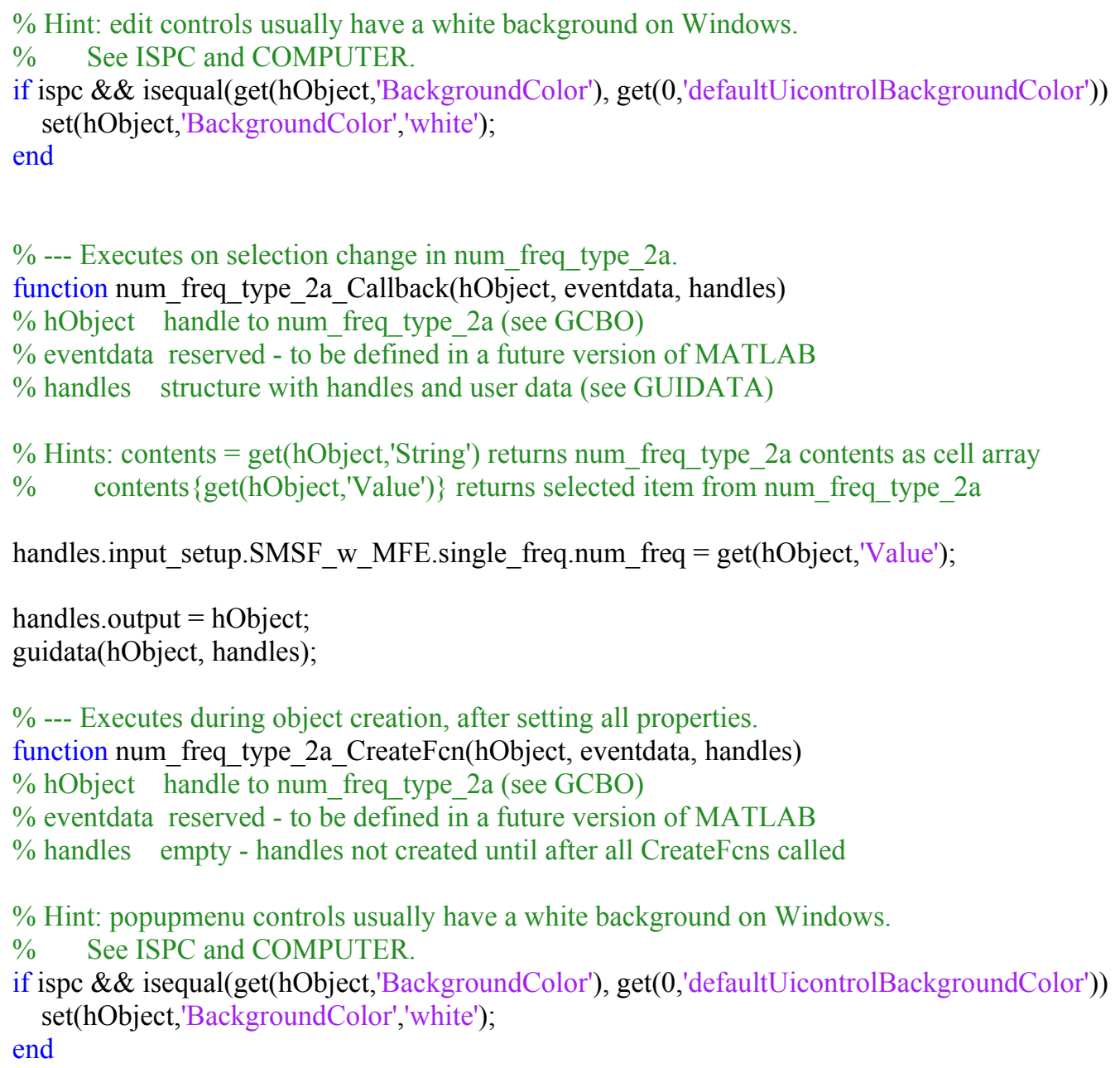


$\% \quad$ See ISPC and COMPUTER.

if ispc \&\& isequal(get(hObject,'BackgroundColor'), get(0,'defaultUicontrolBackgroundColor')) set(hObject,'BackgroundColor','white');

end

function freq_4_type_2a_Callback(hObject, eventdata, handles)

$\%$ hObject handle to freq_4 type_2a (see GCBO)

$\%$ eventdata reserved - to be defined in a future version of MATLAB

$\%$ handles structure with handles and user data (see GUIDATA)

$\%$ Hints: get(hObject,'String') returns contents of freq_4_type_2a as text

$\% \quad$ str2double(get(hObject,'String')) returns contents of freq_4_type_2a as a double

handles.input_setup.SMSF_w_MFE.single_freq.freq(4) = str2double(get(hObject,'String'));

handles.output $=$ hObject;

guidata(hObject, handles);

$\%$--- Executes during object creation, after setting all properties.

function freq_4 type_2a_CreateFcn(hObject, eventdata, handles)

$\%$ hObject handle to freq_4 type_2a (see GCBO)

$\%$ eventdata reserved - to be defined in a future version of MATLAB

$\%$ handles empty - handles not created until after all CreateFcns called

$\%$ Hint: edit controls usually have a white background on Windows.

$\% \quad$ See ISPC and COMPUTER.

if ispc \&\& isequal(get(hObject,'BackgroundColor'), get(0,'defaultUicontrolBackgroundColor')) set(hObject,'BackgroundColor','white'); end

function freq_5 type_2a_Callback(hObject, eventdata, handles)

$\%$ hObject handle to freq 5 type 2a (see GCBO)

$\%$ eventdata reserved - to be defined in a future version of MATLAB

$\%$ handles structure with handles and user data (see GUIDATA)

$\%$ Hints: get(hObject,'String') returns contents of freq 5 type 2 a as text

$\% \quad$ str2double(get(hObject,'String')) returns contents of freq 5 type_2a as a double

handles.input_setup.SMSF_w_MFE.single_freq.freq(5)= str2double(get(hObject,'String'));

handles.output $=$ hObject;

guidata(hObject, handles);

$\%$--- Executes during object creation, after setting all properties.

function freq_5_type_2a_CreateFcn(hObject, eventdata, handles)

$\%$ hObject handle to freq_5 type_2a (see GCBO)

$\%$ eventdata reserved - to be defined in a future version of MATLAB

$\%$ handles empty - handles not created until after all CreateFcns called

$\%$ Hint: edit controls usually have a white background on Windows.

$\% \quad$ See ISPC and COMPUTER.

if ispc \&\& isequal(get(hObject,'BackgroundColor'), get(0,'defaultUicontrolBackgroundColor')) 
set(hObject,'BackgroundColor','white'); end

function freq_6_type_2a_Callback(hObject, eventdata, handles)

$\%$ hObject handle to freq_6_type_2a (see GCBO)

$\%$ eventdata reserved - to be defined in a future version of MATLAB

$\%$ handles structure with handles and user data (see GUIDATA)

$\%$ Hints: get(hObject,'String') returns contents of freq_6_type_2a as text

$\% \quad$ str2double(get(hObject,'String')) returns contents of freq_6_type_2a as a double

handles.input_setup.SMSF_w_MFE.single_freq.freq(6) = str2double(get(hObject,'String'));

handles.output $=$ hObject;

guidata(hObject, handles);

$\%$--- Executes during object creation, after setting all properties.

function freq_6_type_2a_CreateFcn(hObject, eventdata, handles)

$\%$ hObject handle to freq_6 type_2a (see GCBO)

$\%$ eventdata reserved - to be defined in a future version of MATLAB

$\%$ handles empty - handles not created until after all CreateFcns called

$\%$ Hint: edit controls usually have a white background on Windows.

$\% \quad$ See ISPC and COMPUTER.

if ispc \&\& isequal(get(hObject,'BackgroundColor'), get(0,'defaultUicontrolBackgroundColor')) set(hObject,'BackgroundColor','white');

end

function num_samples_type_2b_Callback(hObject, eventdata, handles)

$\%$ hObject handle to num_samples_type_2b (see GCBO)

$\%$ eventdata reserved - to be defined in a future version of MATLAB

$\%$ handles structure with handles and user data (see GUIDATA)

$\%$ Hints: get(hObject,'String') returns contents of num_samples_type_2b as text

$\% \quad$ str2double(get(hObject,'String')) returns contents of num_samples_type_2b as a double

handles.input_setup.SMSF_w_MFE.mult_freq.num_samples = str2double(get(hObject,'String'));

handles.output $=$ hObject;

guidata(hObject, handles);

$\%$--- Executes during object creation, after setting all properties.

function num_samples_type_2b_CreateFcn(hObject, eventdata, handles)

$\%$ hObject handle to num_samples_type_2b (see GCBO)

$\%$ eventdata reserved - to be defined in a future version of MATLAB

$\%$ handles empty - handles not created until after all CreateFcns called

$\%$ Hint: edit controls usually have a white background on Windows.

$\% \quad$ See ISPC and COMPUTER.

if ispc \&\& isequal(get(hObject,'BackgroundColor'), get(0,'defaultUicontrolBackgroundColor'))

set(hObject,'BackgroundColor','white');

end 
function amplitude_type_2b_Callback(hObject, eventdata, handles)

$\%$ hObject handle to amplitude_type_2b (see GCBO)

$\%$ eventdata reserved - to be defined in a future version of MATLAB

$\%$ handles structure with handles and user data (see GUIDATA)

$\%$ Hints: get(hObject,'String') returns contents of amplitude type $2 \mathrm{~b}$ as text

$\% \quad$ str2double(get(hObject,'String')) returns contents of amplitude_type_2b as a double

handles.input_setup.SMSF_w_MFE.mult_freq.amplitude = str2double(get(hObject,'String'));

handles.output $=$ hObject;

guidata(hObject, handles);

$\%$--- Executes during object creation, after setting all properties.

function amplitude_type_2b_CreateFcn(hObject, eventdata, handles)

$\%$ hObject handle to amplitude_type_2b (see GCBO)

$\%$ eventdata reserved - to be defined in a future version of MATLAB

$\%$ handles empty - handles not created until after all CreateFcns called

$\%$ Hint: edit controls usually have a white background on Windows.

$\% \quad$ See ISPC and COMPUTER.

if ispc \&\& isequal(get(hObject,'BackgroundColor'), get( 0 ,'defaultUicontrolBackgroundColor')) set(hObject,'BackgroundColor','white'); end

function freq_1_type_2b_Callback(hObject, eventdata, handles)

$\%$ hObject handle to freq_1_type_2b (see GCBO)

$\%$ eventdata reserved - to be defined in a future version of MATLAB

$\%$ handles structure with handles and user data (see GUIDATA)

$\%$ Hints: get(hObject,'String') returns contents of freq_1_type_2b as text

$\% \quad$ str2double(get(hObject,'String')) returns contents of freq_1_type_2b as a double

handles.input_setup.SMSF_w_MFE.mult_freq.freq(1) = str2double(get(hObject,'String'));

handles.output $=$ hObject;

guidata(hObject, handles);

$\%$--- Executes during object creation, after setting all properties.

function freq_1_type_2b_CreateFcn(hObject, eventdata, handles)

$\%$ hObject handle to freq_1_type_2b (see GCBO)

$\%$ eventdata reserved - to be defined in a future version of MATLAB

$\%$ handles empty - handles not created until after all CreateFcns called

$\%$ Hint: edit controls usually have a white background on Windows.

$\% \quad$ See ISPC and COMPUTER.

if ispc \&\& isequal(get(hObject,'BackgroundColor'), get(0,'defaultUicontrolBackgroundColor')) set(hObject,'BackgroundColor','white'); end 
function freq_2_type_2b_Callback(hObject, eventdata, handles)

$\%$ hObject handle to freq 2 type $2 \mathrm{~b}$ (see GCBO)

$\%$ eventdata reserved - to be defined in a future version of MATLAB

$\%$ handles structure with handles and user data (see GUIDATA)

$\%$ Hints: get(hObject,'String') returns contents of freq 2 type $2 \mathrm{~b}$ as text

$\% \quad$ str2double(get(hObject,'String')) returns contents of freq 2 type $2 \mathrm{~b}$ as a double

handles.input_setup.SMSF_w_MFE.mult_freq.freq(2) = str2double(get(hObject,'String'));

handles.output $=$ hObject;

guidata(hObject, handles);

$\%$--- Executes during object creation, after setting all properties.

function freq_2 type_2b_CreateFcn(hObject, eventdata, handles)

$\%$ hObject handle to freq_2 type_2b (see GCBO)

$\%$ eventdata reserved - to be defined in a future version of MATLAB

$\%$ handles empty - handles not created until after all CreateFcns called

$\%$ Hint: edit controls usually have a white background on Windows.

$\% \quad$ See ISPC and COMPUTER.

if ispc \&\& isequal(get(hObject,'BackgroundColor'), get( 0 ,'defaultUicontrolBackgroundColor')) set(hObject,'BackgroundColor','white'); end

$\%$--- Executes on selection change in num freq type $2 \mathrm{~b}$.

function num_freq_type_2b_Callback(hObject, eventdata, handles)

$\%$ hObject handle to num freq type_2b (see GCBO)

$\%$ eventdata reserved - to be defined in a future version of MATLAB

$\%$ handles structure with handles and user data (see GUIDATA)

$\%$ Hints: contents $=$ get $($ hObject,'String') returns num_freq type $2 \mathrm{~b}$ contents as cell array

$\% \quad$ contents $\{$ get(hObject,'Value') $\}$ returns selected item from num freq type $2 b$

handles.input_setup.SMSF_w_MFE.mult_freq.num_freq = get(hObject,'Value');

handles.output $=$ hObject;

guidata(hObject, handles);

$\%$--- Executes during object creation, after setting all properties.

function num_freq type_2b_CreateFcn(hObject, eventdata, handles)

$\%$ hObject handle to num_freq type_2b (see GCBO)

$\%$ eventdata reserved - to be defined in a future version of MATLAB

$\%$ handles empty - handles not created until after all CreateFcns called

$\%$ Hint: popupmenu controls usually have a white background on Windows.

$\% \quad$ See ISPC and COMPUTER.

if ispc \&\& isequal(get(hObject,'BackgroundColor'), get( 0 ,'defaultUicontrolBackgroundColor')) set(hObject,'BackgroundColor','white'); end

function freq_3_type_2b_Callback(hObject, eventdata, handles) 


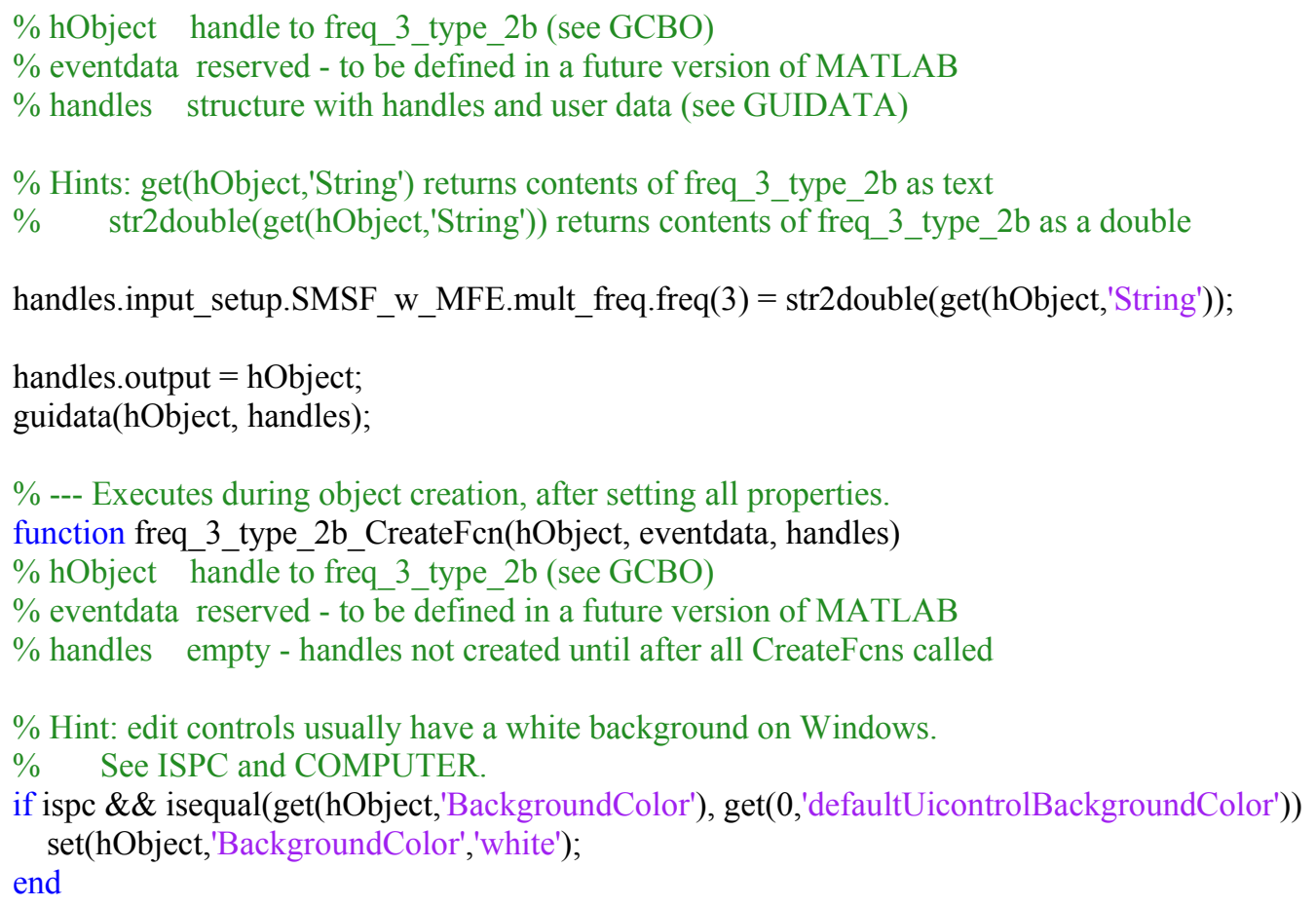




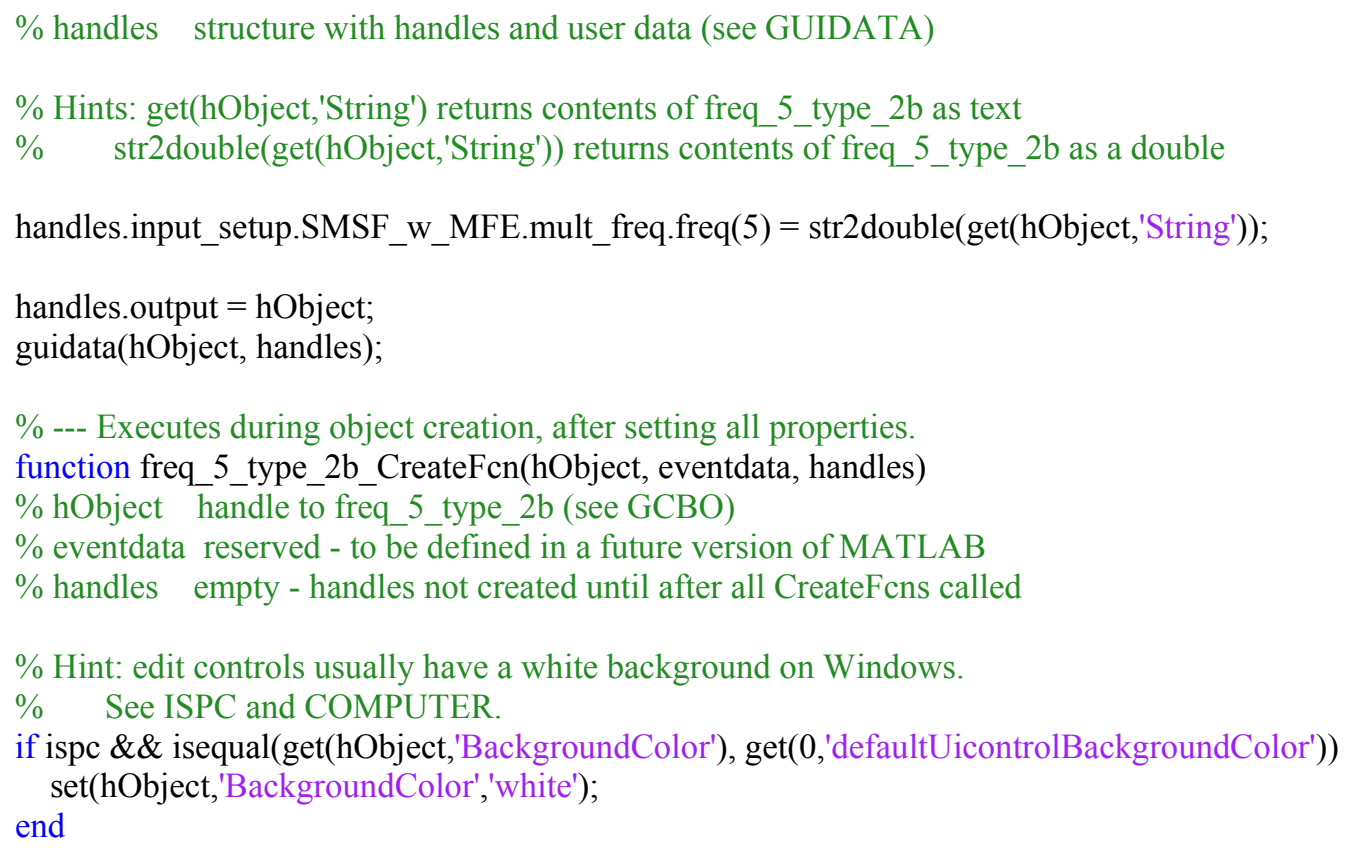

function num_samples_type_3_Callback(hObject, eventdata, handles)

$\%$ hObject handle to num_samples_type_3 (see GCBO)

$\%$ eventdata reserved - to be defined in a future version of MATLAB

$\%$ handles structure with handles and user data (see GUIDATA) 
\% Hints: get(hObject,'String') returns contents of num_samples_type_3 as text

$\% \quad$ str2double(get(hObject,'String')) returns contents of num_samples_type_3 as a double

handles.input_setup.MFW.num_samples = str2double(get(hObject,'String'));

handles.output $=$ hObject;

guidata(hObject, handles);

$\%$--- Executes during object creation, after setting all properties.

function num_samples_type_3_CreateFcn(hObject, eventdata, handles)

$\%$ hObject handle to num_samples_type_3 (see GCBO)

$\%$ eventdata reserved - to be defined in a future version of MATLAB

$\%$ handles empty - handles not created until after all CreateFcns called

$\%$ Hint: edit controls usually have a white background on Windows.

$\% \quad$ See ISPC and COMPUTER.

if ispc \&\& isequal(get(hObject,'BackgroundColor'), get(0,'defaultUicontrolBackgroundColor')) set(hObject,'BackgroundColor','white'); end

function amplitude_type_3_Callback(hObject, eventdata, handles)

$\%$ hObject handle to amplitude_type_3 (see GCBO)

$\%$ eventdata reserved - to be defined in a future version of MATLAB

$\%$ handles structure with handles and user data (see GUIDATA)

$\%$ Hints: get(hObject,'String') returns contents of amplitude_type_3 as text

$\% \quad$ str2double(get(hObject,'String')) returns contents of amplitude_type_3 as a double

handles.input_setup.MFW.amplitude = str2double(get(hObject,'String'));

handles.output $=$ hObject;

guidata(hObject, handles);

$\%$--- Executes during object creation, after setting all properties.

function amplitude_type_3_CreateFcn(hObject, eventdata, handles)

$\%$ hObject handle to amplitude_type_3 (see GCBO)

$\%$ eventdata reserved - to be defined in a future version of MATLAB

$\%$ handles empty - handles not created until after all CreateFcns called

$\%$ Hint: edit controls usually have a white background on Windows.

$\% \quad$ See ISPC and COMPUTER.

if ispc \&\& isequal(get(hObject,'BackgroundColor'), get(0,'defaultUicontrolBackgroundColor')) set(hObject,'BackgroundColor','white'); end

function freq_1_type_3_Callback(hObject, eventdata, handles)

$\%$ hObject handle to freq_1_type_3 (see GCBO)

$\%$ eventdata reserved - to be defined in a future version of MATLAB

$\%$ handles structure with handles and user data (see GUIDATA)

\% Hints: get(hObject,'String') returns contents of freq_1_type_3 as text

$\% \quad$ str2double(get(hObject,'String')) returns contents of freq_1_type_3 as a double 


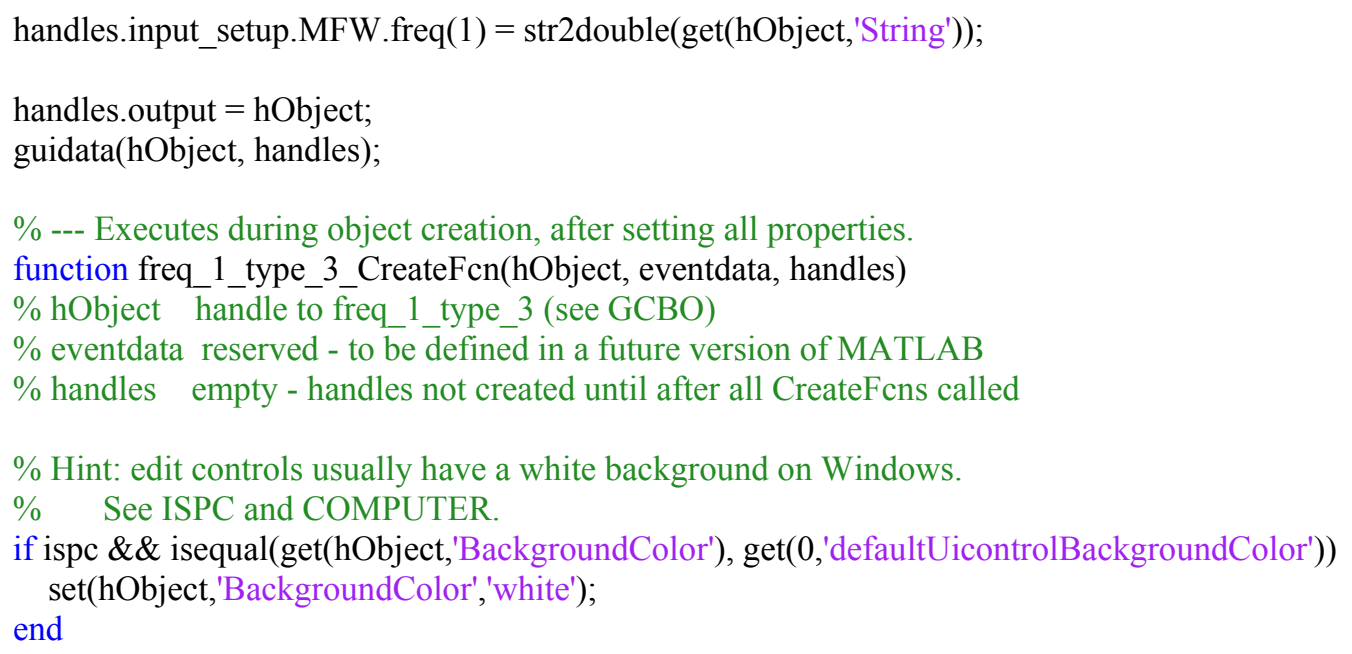


handles.output $=$ hObject;
guidata(hObject, handles);

$\%$--- Executes during object creation, after setting all properties.

function num_freq_type_3_CreateFcn(hObject, eventdata, handles)

$\%$ hObject handle to num freq type 3 (see GCBO)

$\%$ eventdata reserved - to be defined in a future version of MATLAB

$\%$ handles empty - handles not created until after all CreateFcns called

$\%$ Hint: popupmenu controls usually have a white background on Windows.

$\% \quad$ See ISPC and COMPUTER.

if ispc \&\& isequal(get(hObject,'BackgroundColor'), get( 0 ,'defaultUicontrolBackgroundColor'))

set(hObject,'BackgroundColor','white'); end

function freq_3_type_3_Callback(hObject, eventdata, handles)

$\%$ hObject handle to freq_3 type_3 (see GCBO)

$\%$ eventdata reserved - to be defined in a future version of MATLAB

$\%$ handles structure with handles and user data (see GUIDATA)

$\%$ Hints: get(hObject,'String') returns contents of freq_3_type_3 as text

$\% \quad$ str2double(get(hObject,'String')) returns contents of freq_3 type_3 as a double

handles.input_setup.MFW.freq(3) = str2double(get(hObject,'String'));

handles.output $=$ hObject;

guidata(hObject, handles);

$\%$--- Executes during object creation, after setting all properties.

function freq_3_type_3_CreateFcn(hObject, eventdata, handles)

$\%$ hObject handle to freq 3 type 3 (see GCBO)

$\%$ eventdata reserved - to be defined in a future version of MATLAB

$\%$ handles empty - handles not created until after all CreateFcns called

$\%$ Hint: edit controls usually have a white background on Windows.

$\% \quad$ See ISPC and COMPUTER.

if ispc \&\& isequal(get(hObject,'BackgroundColor'), get(0,'defaultUicontrolBackgroundColor')) set(hObject,'BackgroundColor','white');

end

function freq_4_type_3_Callback(hObject, eventdata, handles)

$\%$ hObject handle to freq 4 type_3 (see GCBO)

$\%$ eventdata reserved - to be defined in a future version of MATLAB

$\%$ handles structure with handles and user data (see GUIDATA)

$\%$ Hints: get(hObject,'String') returns contents of freq_4_type_3 as text

$\% \quad$ str2double(get(hObject,'String')) returns contents of freq_4_type_3 as a double

handles.input_setup.MFW.freq(4) = str2double(get(hObject,'String'));

handles.output $=$ hObject; 
guidata(hObject, handles);

$\%$--- Executes during object creation, after setting all properties.

function freq_4_type_3_CreateFcn(hObject, eventdata, handles)

$\%$ hObject handle to freq 4 type_3 (see GCBO)

$\%$ eventdata reserved - to be defined in a future version of MATLAB

$\%$ handles empty - handles not created until after all CreateFcns called

$\%$ Hint: edit controls usually have a white background on Windows.

$\% \quad$ See ISPC and COMPUTER.

if ispc \&\& isequal(get(hObject,'BackgroundColor'), get( 0 ,'defaultUicontrolBackgroundColor')) set(hObject,'BackgroundColor','white'); end

function freq_5_type_3_Callback(hObject, eventdata, handles)

$\%$ hObject handle to freq 5 type_3 (see GCBO)

$\%$ eventdata reserved - to be defined in a future version of MATLAB

$\%$ handles structure with handles and user data (see GUIDATA)

$\%$ Hints: get(hObject,'String') returns contents of freq 5 type 3 as text

$\% \quad$ str2double(get(hObject,'String')) returns contents of freq_5_type_3 as a double

handles.input_setup.MFW.freq(5) = str2double(get(hObject,'String'));

handles.output $=$ hObject;

guidata(hObject, handles);

$\%$--- Executes during object creation, after setting all properties.

function freq_5_type_3_CreateFcn(hObject, eventdata, handles)

$\%$ hObject handle to freq 5 type_3 (see GCBO)

$\%$ eventdata reserved - to be defined in a future version of MATLAB

$\%$ handles empty - handles not created until after all CreateFcns called

$\%$ Hint: edit controls usually have a white background on Windows.

$\% \quad$ See ISPC and COMPUTER.

if ispc \&\& isequal(get(hObject,'BackgroundColor'), get( 0 ,'defaultUicontrolBackgroundColor')) set(hObject,'BackgroundColor','white'); end

function freq_6_type_3_Callback(hObject, eventdata, handles)

$\%$ hObject handle to freq_6_type_3 (see GCBO)

$\%$ eventdata reserved - to be defined in a future version of MATLAB

$\%$ handles structure with handles and user data (see GUIDATA)

\% Hints: get(hObject,'String') returns contents of freq_6_type_3 as text

$\% \quad$ str2double(get(hObject,'String')) returns contents of freq_6 type_3 as a double

handles.input_setup.MFW.freq(6) = str2double(get(hObject,'String'));

handles.output $=$ hObject;

guidata(hObject, handles); 


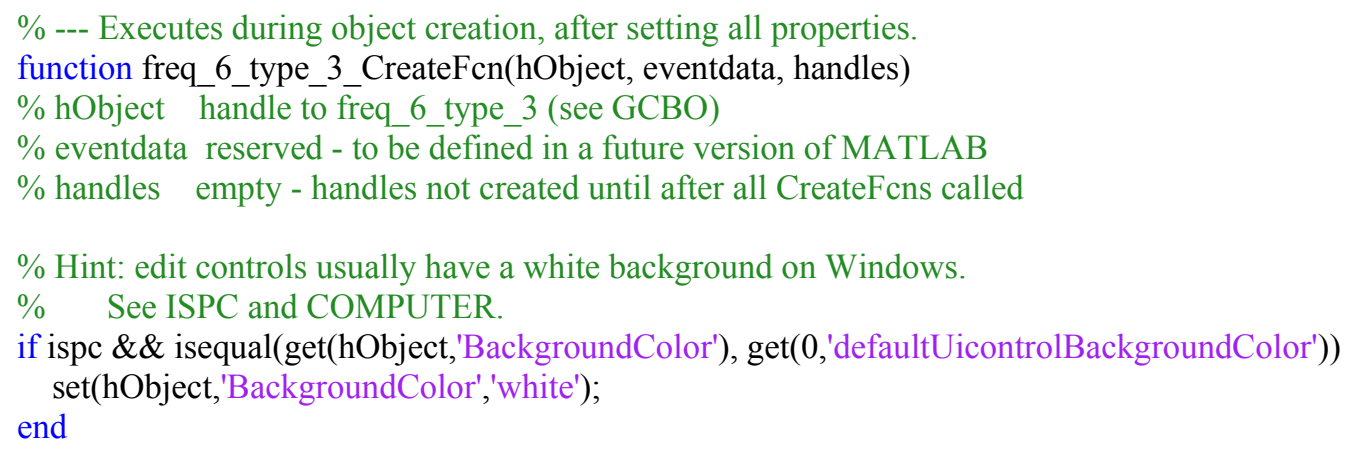

$\%$--- Executes during object creation, after setting all properties.

function time_type_4_CreateFcn(hObject, eventdata, handles) 
$\%$ hObject handle to time_type_4 (see GCBO)

$\%$ eventdata reserved - to be defined in a future version of MATLAB

$\%$ handles empty - handles not created until after all CreateFcns called

$\%$ Hint: edit controls usually have a white background on Windows.

$\% \quad$ See ISPC and COMPUTER.

if ispc \&\& isequal(get(hObject,'BackgroundColor'), get(0,'defaultUicontrolBackgroundColor')) set(hObject,'BackgroundColor','white'); end

function num_samples_type_5_Callback(hObject, eventdata, handles)

$\%$ hObject handle to num_samples_type_5 (see GCBO)

$\%$ eventdata reserved - to be defined in a future version of MATLAB

$\%$ handles structure with handles and user data (see GUIDATA)

$\%$ Hints: get(hObject,'String') returns contents of num_samples type 5 as text

$\% \quad$ str2double(get(hObject,'String')) returns contents of num_samples_type_5 as a double

handles.input_setup.NSCRS.num_samples $=$ str2double $($ get $($ hObject,'String') $)$;

handles.output $=$ hObject;

guidata(hObject, handles);

$\%$--- Executes during object creation, after setting all properties.

function num_samples_type_5_CreateFcn(hObject, eventdata, handles)

$\%$ hObject handle to num samples type 5 (see GCBO)

$\%$ eventdata reserved - to be defined in a future version of MATLAB

$\%$ handles empty - handles not created until after all CreateFcns called

$\%$ Hint: edit controls usually have a white background on Windows.

$\% \quad$ See ISPC and COMPUTER.

if ispc \&\& isequal(get(hObject,'BackgroundColor'), get( 0 ,'defaultUicontrolBackgroundColor')) set(hObject,'BackgroundColor','white');

end

function min_amplitude type 5_Callback(hObject, eventdata, handles)

$\%$ hObject handle to min_amplitude type 5 (see GCBO)

$\%$ eventdata reserved - to be defined in a future version of MATLAB

$\%$ handles structure with handles and user data (see GUIDATA)

$\%$ Hints: get(hObject,'String') returns contents of min_amplitude_type_5 as text

$\% \quad$ str2double(get(hObject,'String')) returns contents of min_amplitude_type_5 as a double

handles.input_setup.NSCRS.min_amp = str2double(get(hObject,'String'));

handles.output $=$ hObject;

guidata(hObject, handles);

$\%$--- Executes during object creation, after setting all properties.

function min_amplitude_type_5_CreateFcn(hObject, eventdata, handles)

$\%$ hObject handle to min_amplitude type 5 (see GCBO)

$\%$ eventdata reserved - to be defined in a future version of MATLAB 
$\%$ handles empty - handles not created until after all CreateFcns called

$\%$ Hint: edit controls usually have a white background on Windows.

$\% \quad$ See ISPC and COMPUTER.

if ispc \&\& isequal(get(hObject,'BackgroundColor'), get( 0 ,'defaultUicontrolBackgroundColor')) set(hObject,'BackgroundColor','white'); end

function max_amplitude_type_5_Callback(hObject, eventdata, handles)

$\%$ hObject handle to max_amplitude_type_5 (see GCBO)

$\%$ eventdata reserved - to be defined in a future version of MATLAB

$\%$ handles structure with handles and user data (see GUIDATA)

$\%$ Hints: get(hObject,'String') returns contents of max_amplitude_type_5 as text

$\% \quad$ str2double(get(hObject,'String')) returns contents of max_amplitude_type 5 as a double

handles.input_setup.NSCRS.max_amp = str2double(get(hObject,'String'));

handles.output $=$ hObject;

guidata(hObject, handles);

$\%$--- Executes during object creation, after setting all properties.

function max_amplitude_type 5_CreateFcn(hObject, eventdata, handles)

$\%$ hObject handle to max_amplitude_type_5 (see GCBO)

$\%$ eventdata reserved - to be defined in a future version of MATLAB

$\%$ handles empty - handles not created until after all CreateFcns called

$\%$ Hint: edit controls usually have a white background on Windows.

$\% \quad$ See ISPC and COMPUTER.

if ispc \&\& isequal(get(hObject,'BackgroundColor'), get(0,'defaultUicontrolBackgroundColor')) set(hObject,'BackgroundColor','white'); end

function interval_type_5_Callback(hObject, eventdata, handles)

$\%$ hObject handle to interval_type_5 (see GCBO)

$\%$ eventdata reserved - to be defined in a future version of MATLAB

$\%$ handles structure with handles and user data (see GUIDATA)

$\%$ Hints: get(hObject,'String') returns contents of interval_type 5 as text

$\% \quad$ str2double(get(hObject,'String')) returns contents of interval_type_5 as a double

handles.input_setup.NSCRS.interval = str2double $($ get $($ hObject,'String' $))$;

handles.output $=$ hObject;

guidata(hObject, handles);

$\%$--- Executes during object creation, after setting all properties.

function interval_type_5_CreateFcn(hObject, eventdata, handles)

$\%$ hObject handle to interval type_5 (see GCBO)

$\%$ eventdata reserved - to be defined in a future version of MATLAB

$\%$ handles empty - handles not created until after all CreateFcns called 
$\%$ Hint: edit controls usually have a white background on Windows.

$\% \quad$ See ISPC and COMPUTER.

if ispc \&\& isequal(get(hObject,'BackgroundColor'), get(0,'defaultUicontrolBackgroundColor')) set(hObject,'BackgroundColor','white');

end

function num_samples_type_6_Callback(hObject, eventdata, handles)

$\%$ hObject handle to num_samples_type_6 (see GCBO)

$\%$ eventdata reserved - to be defined in a future version of MATLAB

$\%$ handles structure with handles and user data (see GUIDATA)

$\%$ Hints: get(hObject,'String') returns contents of num_samples_type_6 as text

$\% \quad$ str2double(get(hObject,'String')) returns contents of num_samples type 6 as a double

handles.input_setup.RSLC.num_samples = str2double(get(hObject,'String'));

handles.output $=$ hObject;

guidata(hObject, handles);

$\%$--- Executes during object creation, after setting all properties.

function num_samples_type_6_CreateFcn(hObject, eventdata, handles)

$\%$ hObject handle to num_samples_type_6 (see GCBO)

$\%$ eventdata reserved - to be defined in a future version of MATLAB

$\%$ handles empty - handles not created until after all CreateFcns called

$\%$ Hint: edit controls usually have a white background on Windows.

$\% \quad$ See ISPC and COMPUTER.

if ispc \&\& isequal(get(hObject,'BackgroundColor'), get(0,'defaultUicontrolBackgroundColor')) set(hObject,'BackgroundColor','white'); end

function min_amplitude_type_6_Callback(hObject, eventdata, handles)

$\%$ hObject handle to min_amplitude_type_6 (see GCBO)

$\%$ eventdata reserved - to be defined in a future version of MATLAB

$\%$ handles structure with handles and user data (see GUIDATA)

$\%$ Hints: get(hObject,'String') returns contents of min_amplitude type_6 as text

$\% \quad$ str2double(get(hObject,'String')) returns contents of min_amplitude_type_6 as a double

handles.input_setup.RSLC.min_amp = str2double(get(hObject,'String'));

handles.output $=$ hObject;

guidata(hObject, handles);

$\%$--- Executes during object creation, after setting all properties.

function min_amplitude_type_6_CreateFcn(hObject, eventdata, handles)

$\%$ hObject handle to min_amplitude_type_6 (see GCBO)

$\%$ eventdata reserved - to be defined in a future version of MATLAB

$\%$ handles empty - handles not created until after all CreateFcns called

$\%$ Hint: edit controls usually have a white background on Windows.

$\% \quad$ See ISPC and COMPUTER. 
if ispc \&\& isequal(get(hObject,'BackgroundColor'), get(0,'defaultUicontrolBackgroundColor')) set(hObject,'BackgroundColor','white'); end

function max_amplitude_type_6_Callback(hObject, eventdata, handles)

$\%$ hObject handle to max amplitude type 6 (see GCBO)

$\%$ eventdata reserved - to be defined in a future version of MATLAB

$\%$ handles structure with handles and user data (see GUIDATA)

$\%$ Hints: get(hObject,'String') returns contents of max_amplitude_type_6 as text

$\% \quad$ str2double(get(hObject,'String')) returns contents of max_amplitude_type_6 as a double

handles.input_setup.RSLC.max_amp = str2double(get(hObject,'String'));

handles.output $=$ hObject;

guidata(hObject, handles);

$\%$--- Executes during object creation, after setting all properties.

function max_amplitude_type_6_CreateFcn(hObject, eventdata, handles)

$\%$ hObject handle to max amplitude type 6 (see GCBO)

$\%$ eventdata reserved - to be defined in a future version of MATLAB

$\%$ handles empty - handles not created until after all CreateFcns called

$\%$ Hint: edit controls usually have a white background on Windows.

$\% \quad$ See ISPC and COMPUTER.

if ispc \&\& isequal(get(hObject,'BackgroundColor'), get(0,'defaultUicontrolBackgroundColor')) set(hObject,'BackgroundColor','white');

end

function min_interval_type_6_Callback(hObject, eventdata, handles)

$\%$ hObject handle to min interval type 6 (see GCBO)

$\%$ eventdata reserved - to be defined in a future version of MATLAB

$\%$ handles structure with handles and user data (see GUIDATA)

$\%$ Hints: get(hObject,'String') returns contents of min_interval_type_6 as text

$\% \quad$ str2double(get(hObject,'String')) returns contents of min interval type 6 as a double

handles.input_setup.RSLC.min_interval =str2double $($ get $($ hObject,'String' $))$;

handles.output $=$ hObject;

guidata(hObject, handles);

$\%$--- Executes during object creation, after setting all properties.

function min_interval_type_6_CreateFcn(hObject, eventdata, handles)

$\%$ hObject handle to min_interval_type_6 (see GCBO)

$\%$ eventdata reserved - to be defined in a future version of MATLAB

$\%$ handles empty - handles not created until after all CreateFcns called

$\%$ Hint: edit controls usually have a white background on Windows.

$\% \quad$ See ISPC and COMPUTER.

if ispc \&\& isequal(get(hObject,'BackgroundColor'), get(0,'defaultUicontrolBackgroundColor')) set(hObject,'BackgroundColor','white'); 
end

function max interval_type_6_Callback(hObject, eventdata, handles)

$\%$ hObject handle to max_interval_type_6 (see GCBO)

$\%$ eventdata reserved - to be defined in a future version of MATLAB

$\%$ handles structure with handles and user data (see GUIDATA)

$\%$ Hints: get(hObject,'String') returns contents of max_interval_type_6 as text

$\% \quad$ str2double(get(hObject,'String')) returns contents of max_interval_type_6 as a double

handles.input_setup.RSLC.max_interval = str2double $($ get(hObject,'String'));

handles.output $=$ hObject;

guidata(hObject, handles);

$\%$--- Executes during object creation, after setting all properties.

function max_interval_type_6_CreateFcn(hObject, eventdata, handles)

$\%$ hObject handle to max_interval_type_6 (see GCBO)

$\%$ eventdata reserved - to be defined in a future version of MATLAB

$\%$ handles empty - handles not created until after all CreateFcns called

$\%$ Hint: edit controls usually have a white background on Windows.

$\% \quad$ See ISPC and COMPUTER.

if ispc \&\& isequal(get(hObject,'BackgroundColor'), get(0,'defaultUicontrolBackgroundColor')) set(hObject,'BackgroundColor','white'); end

$\%$--- Executes on selection change in Segments type 7.

function Segments_type_7_Callback(hObject, eventdata, handles)

$\%$ hObject handle to Segments_type_7 (see GCBO)

$\%$ eventdata reserved - to be defined in a future version of MATLAB

$\%$ handles structure with handles and user data (see GUIDATA)

$\%$ Hints: contents $=$ get $($ hObject,'String') returns Segments type 7 contents as cell array

$\% \quad$ contents $\{$ get(hObject,'Value')\} returns selected item from Segments_type_7

handles.input_setup.RSLC_w_DAS.num_segments = get(hObject,'Value');

handles.output $=$ hObject;

guidata(hObject, handles);

$\%$--- Executes during object creation, after setting all properties.

function Segments_type_7_CreateFcn(hObject, eventdata, handles)

$\%$ hObject handle to Segments type 7 (see GCBO)

$\%$ eventdata reserved - to be defined in a future version of MATLAB

$\%$ handles empty - handles not created until after all CreateFcns called

$\%$ Hint: popupmenu controls usually have a white background on Windows.

$\% \quad$ See ISPC and COMPUTER.

if ispc \&\& isequal(get(hObject,'BackgroundColor'), get(0,'defaultUicontrolBackgroundColor')) set(hObject,'BackgroundColor','white');

end 
function min_seg2_type_7_Callback(hObject, eventdata, handles)

$\%$ hObject handle to min seg2 type 7 (see GCBO)

$\%$ eventdata reserved - to be defined in a future version of MATLAB

$\%$ handles structure with handles and user data (see GUIDATA)

$\%$ Hints: get(hObject,'String') returns contents of min_seg2 type 7 as text

$\% \quad$ str2double(get(hObject,'String')) returns contents of min seg2 type 7 as a double

handles.input_setup.RSLC_w_DAS.seg(2).min_amp = str2double(get(hObject,'String'));

handles.output $=$ hObject;

guidata(hObject, handles);

$\%$--- Executes during object creation, after setting all properties.

function min_seg2_type_7_CreateFcn(hObject, eventdata, handles)

$\%$ hObject handle to min seg2 type 7 (see GCBO)

$\%$ eventdata reserved - to be defined in a future version of MATLAB

$\%$ handles empty - handles not created until after all CreateFcns called

$\%$ Hint: edit controls usually have a white background on Windows.

$\% \quad$ See ISPC and COMPUTER.

if ispc \&\& isequal(get(hObject,'BackgroundColor'), get( 0 ,'defaultUicontrolBackgroundColor')) set(hObject,'BackgroundColor','white'); end

function edit46_Callback(hObject, eventdata, handles)

$\%$ hObject handle to edit46 (see GCBO)

$\%$ eventdata reserved - to be defined in a future version of MATLAB

$\%$ handles structure with handles and user data (see GUIDATA)

$\%$ Hints: get(hObject,'String') returns contents of edit46 as text

$\% \quad$ str2double(get(hObject,'String')) returns contents of edit46 as a double

$\%$--- Executes during object creation, after setting all properties.

function edit46_CreateFcn(hObject, eventdata, handles)

$\%$ hObject handle to edit46 (see GCBO)

$\%$ eventdata reserved - to be defined in a future version of MATLAB

$\%$ handles empty - handles not created until after all CreateFcns called

$\%$ Hint: edit controls usually have a white background on Windows.

$\% \quad$ See ISPC and COMPUTER.

if ispc \&\& isequal(get(hObject,'BackgroundColor'), get( 0 ,'defaultUicontrolBackgroundColor')) set(hObject,'BackgroundColor','white');

end

function edit47 Callback(hObject, eventdata, handles)

$\%$ hObject handle to edit47 (see GCBO)

$\%$ eventdata reserved - to be defined in a future version of MATLAB

$\%$ handles structure with handles and user data (see GUIDATA) 


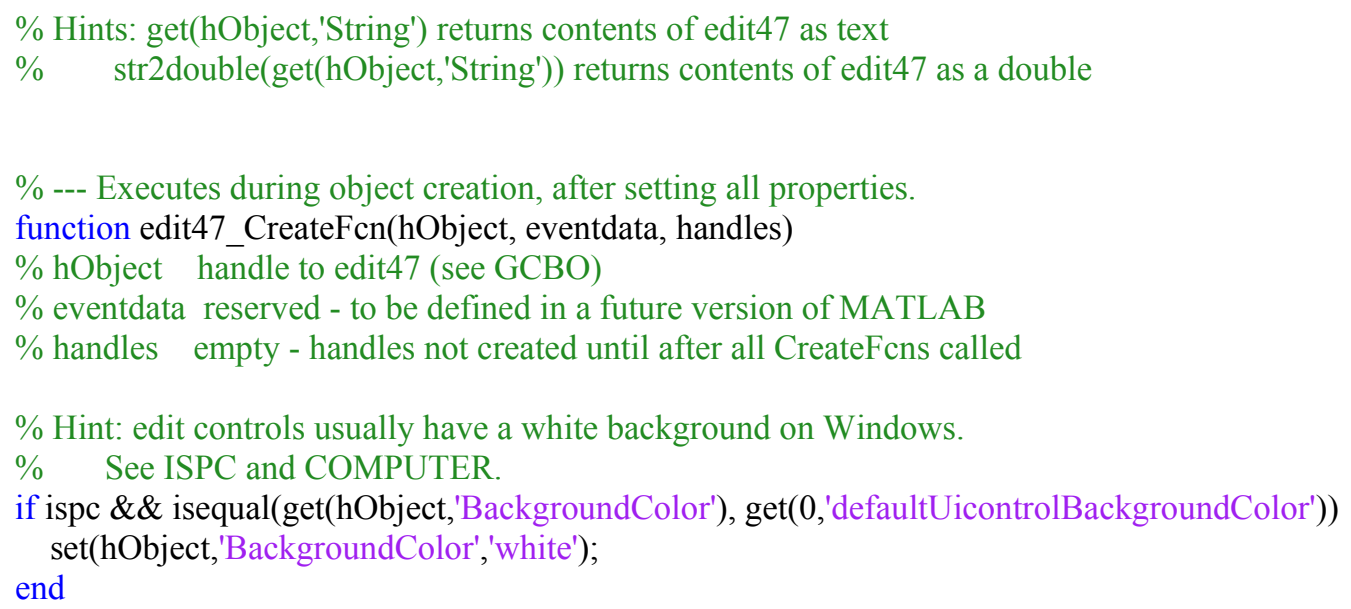


handles.output $=$ hObject;
guidata(hObject, handles);

$\%$--- Executes during object creation, after setting all properties.

function samples_seg_2 type 7_CreateFcn(hObject, eventdata, handles)

$\%$ hObject handle to samples_seg_2_type_7 (see GCBO)

$\%$ eventdata reserved - to be defined in a future version of MATLAB

$\%$ handles empty - handles not created until after all CreateFcns called

$\%$ Hint: edit controls usually have a white background on Windows.

$\% \quad$ See ISPC and COMPUTER.

if ispc \&\& isequal(get(hObject,'BackgroundColor'), get( 0 ,'defaultUicontrolBackgroundColor')) set(hObject,'BackgroundColor','white'); end

function min_interval_type_7_Callback(hObject, eventdata, handles)

$\%$ hObject handle to min_interval_type_7 (see GCBO)

$\%$ eventdata reserved - to be defined in a future version of MATLAB

$\%$ handles structure with handles and user data (see GUIDATA)

$\%$ Hints: get(hObject,'String') returns contents of min_interval_type_7 as text

$\% \quad$ str2double(get(hObject,'String')) returns contents of min_interval_type_7 as a double

handles.input_setup.RSLC_w_DAS.min_interval = str2double $($ get $($ hObject,'String'));

handles.output $=$ hObject;

guidata(hObject, handles);

$\%$--- Executes during object creation, after setting all properties.

function min_interval_type_7_CreateFcn(hObject, eventdata, handles)

$\%$ hObject handle to min_interval_type_7 (see GCBO)

$\%$ eventdata reserved - to be defined in a future version of MATLAB

$\%$ handles empty - handles not created until after all CreateFcns called

$\%$ Hint: edit controls usually have a white background on Windows.

$\% \quad$ See ISPC and COMPUTER.

if ispc \&\& isequal(get(hObject,'BackgroundColor'), get(0,'defaultUicontrolBackgroundColor')) set(hObject,'BackgroundColor','white'); end

function max_interval_type_7_Callback(hObject, eventdata, handles)

$\%$ hObject handle to max_interval_type_7 (see GCBO)

$\%$ eventdata reserved - to be define $\bar{d}$ in a future version of MATLAB

$\%$ handles structure with handles and user data (see GUIDATA)

$\%$ Hints: get(hObject,'String') returns contents of max_interval_type_7 as text

$\% \quad$ str2double(get(hObject,'String')) returns contents of max_interval_type_7 as a double

handles.input_setup.RSLC_w_DAS.max_interval = str2double $($ get $($ hObject,'String' $))$;

handles.output $=$ hObject;

guidata(hObject, handles); 


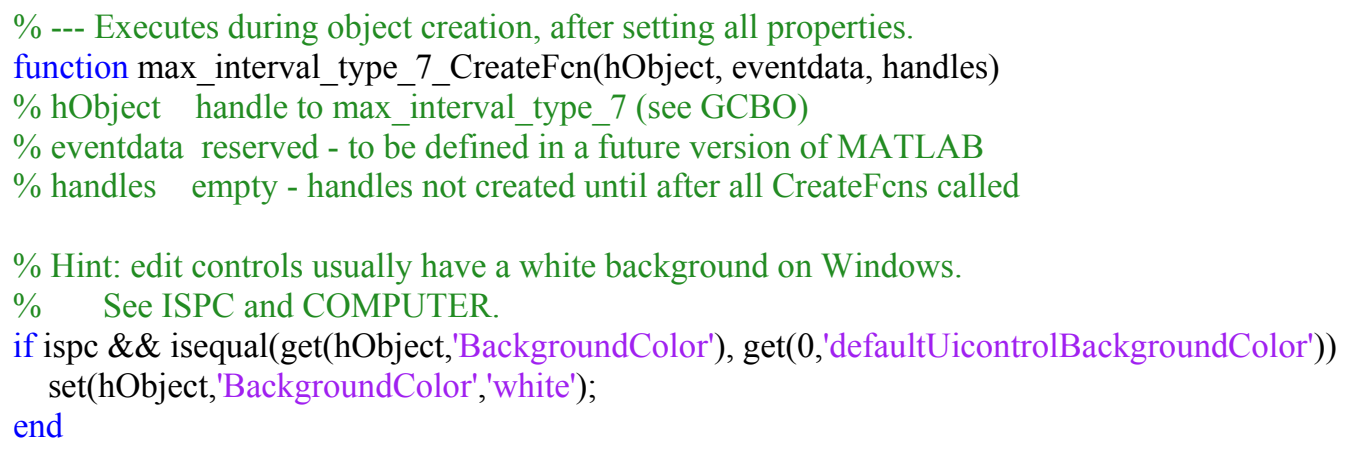


function max_seg1_type_7_CreateFcn(hObject, eventdata, handles)

$\%$ hObject handle to max_seg1_type_7 (see GCBO)

$\%$ eventdata reserved - to be defined in a future version of MATLAB

$\%$ handles empty - handles not created until after all CreateFcns called

$\%$ Hint: edit controls usually have a white background on Windows.

$\% \quad$ See ISPC and COMPUTER.

if ispc \&\& isequal(get(hObject,'BackgroundColor'), get( 0 ,'defaultUicontrolBackgroundColor')) set(hObject,'BackgroundColor','white');

end

function samples_seg_1_type_7_Callback(hObject, eventdata, handles)

$\%$ hObject handle to samples_seg_1_type_7 (see GCBO)

$\%$ eventdata reserved - to be defined in a future version of MATLAB

$\%$ handles structure with handles and user data (see GUIDATA)

$\%$ Hints: get(hObject,'String') returns contents of samples_seg_1_type_7 as text

$\% \quad$ str2double(get(hObject,'String')) returns contents of samples_seg_1_type_7 as a double

handles.input_setup.RSLC_w_DAS.seg(1).num_samples = str2double(get(hObject,'String'));

handles.output $=$ hObject;

guidata(hObject, handles);

$\%$--- Executes during object creation, after setting all properties.

function samples_seg_1 type 7 CreateFcn(hObject, eventdata, handles)

$\%$ hObject handle to samples_seg_1_type_7 (see GCBO)

$\%$ eventdata reserved - to be defined in a future version of MATLAB

$\%$ handles empty - handles not created until after all CreateFcns called

$\%$ Hint: edit controls usually have a white background on Windows.

$\% \quad$ See ISPC and COMPUTER.

if ispc \&\& isequal(get(hObject,'BackgroundColor'), get( 0 ,'defaultUicontrolBackgroundColor'))

set(hObject,'BackgroundColor','white');

end

function min_seg3 type 7_Callback(hObject, eventdata, handles)

$\%$ hObject handle to min_seg3_type_7 (see GCBO)

$\%$ eventdata reserved - to be defined in a future version of MATLAB

$\%$ handles structure with handles and user data (see GUIDATA)

$\%$ Hints: get(hObject,'String') returns contents of min_seg3 type_7 as text

$\% \quad$ str2double(get(hObject,'String')) returns contents of min_seg3 type 7 as a double

handles.input_setup.RSLC_w_DAS.seg(3).min_amp = str2double(get(hObject,'String'));

handles.output $=$ hObject;

guidata(hObject, handles);

$\%$--- Executes during object creation, after setting all properties.

function min seg3 type 7 CreateFcn(hObject, eventdata, handles)

$\%$ hObject handle to min_seg3_type_7 (see GCBO) 
$\%$ eventdata reserved - to be defined in a future version of MATLAB

$\%$ handles empty - handles not created until after all CreateFcns called

$\%$ Hint: edit controls usually have a white background on Windows.

$\% \quad$ See ISPC and COMPUTER.

if ispc \&\& isequal(get(hObject,'BackgroundColor'), get(0,'defaultUicontrolBackgroundColor')) set(hObject,'BackgroundColor','white'); end

function max_seg3_type_7_Callback(hObject, eventdata, handles)

$\%$ hObject handle to max_seg3_type_7 (see GCBO)

$\%$ eventdata reserved - to be defined in a future version of MATLAB

$\%$ handles structure with handles and user data (see GUIDATA)

$\%$ Hints: get(hObject,'String') returns contents of max_seg3_type 7 as text

$\% \quad$ str2double(get(hObject,'String')) returns contents of max seg3 type 7 as a double

handles.input_setup.RSLC_w_DAS.seg(3).max_amp = str2double(get(hObject,'String'));

handles.output $=$ hObject;

guidata(hObject, handles);

$\%$--- Executes during object creation, after setting all properties.

function max_seg3_type_7_CreateFcn(hObject, eventdata, handles)

$\%$ hObject handle to max_seg3_type_7 (see GCBO)

$\%$ eventdata reserved - to be defined in a future version of MATLAB

$\%$ handles empty - handles not created until after all CreateFcns called

$\%$ Hint: edit controls usually have a white background on Windows.

$\% \quad$ See ISPC and COMPUTER.

if ispc \&\& isequal(get(hObject,'BackgroundColor'), get( 0, 'defaultUicontrolBackgroundColor')) set(hObject,'BackgroundColor','white'); end

function samples_seg_3_type_7_Callback(hObject, eventdata, handles)

$\%$ hObject handle to samples_seg_3_type_7 (see GCBO)

$\%$ eventdata reserved - to be defined in a future version of MATLAB

$\%$ handles structure with handles and user data (see GUIDATA)

$\%$ Hints: get(hObject,'String') returns contents of samples_seg_3_type_7 as text

$\% \quad$ str2double(get(hObject,'String')) returns contents of samples_seg_3_type_7 as a double

handles.input_setup.RSLC_w_DAS.seg(3).num_samples = str2double(get(hObject,'String'));

handles.output $=$ hObject;

guidata(hObject, handles);

$\%$--- Executes during object creation, after setting all properties.

function samples_seg_3 type 7_CreateFcn(hObject, eventdata, handles)

$\%$ hObject handle to samples_seg_3 type 7 (see GCBO)

$\%$ eventdata reserved - to be defined in a future version of MATLAB

$\%$ handles empty - handles not created until after all CreateFcns called 
$\%$ Hint: edit controls usually have a white background on Windows.

$\% \quad$ See ISPC and COMPUTER.

if ispc \&\& isequal(get(hObject,'BackgroundColor'), get(0,'defaultUicontrolBackgroundColor')) set(hObject,'BackgroundColor','white'); end

function min_seg4_type_7_Callback(hObject, eventdata, handles)

$\%$ hObject handle to min_seg4_type_7 (see GCBO)

$\%$ eventdata reserved - to be defined in a future version of MATLAB

$\%$ handles structure with handles and user data (see GUIDATA)

$\%$ Hints: get(hObject,'String') returns contents of min_seg4 type 7 as text

$\% \quad$ str2double(get(hObject,'String')) returns contents of min_seg4_type_7 as a double

handles.input_setup.RSLC_w_DAS.seg(4).min_amp = str2double(get(hObject,'String'));

handles.output $=$ hObject;

guidata(hObject, handles);

$\%$--- Executes during object creation, after setting all properties.

function min_seg4_type_7_CreateFcn(hObject, eventdata, handles)

$\%$ hObject handle to min_seg4_type_7 (see GCBO)

$\%$ eventdata reserved - to be defined in a future version of MATLAB

$\%$ handles empty - handles not created until after all CreateFcns called

$\%$ Hint: edit controls usually have a white background on Windows.

$\% \quad$ See ISPC and COMPUTER.

if ispc \&\& isequal(get(hObject,'BackgroundColor'), get(0,'defaultUicontrolBackgroundColor')) set(hObject,'BackgroundColor','white'); end

function max_seg4_type_7_Callback(hObject, eventdata, handles)

$\%$ hObject handle to max_seg4_type_7 (see GCBO)

$\%$ eventdata reserved - to be defined in a future version of MATLAB

$\%$ handles structure with handles and user data (see GUIDATA)

\% Hints: get(hObject,'String') returns contents of max_seg4_type_7 as text

$\% \quad$ str2double(get(hObject,'String')) returns contents of max_seg4_type 7 as a double

handles.input_setup.RSLC_w_DAS.seg(4).max_amp = str2double(get(hObject,'String'));

handles.output $=$ hObject;

guidata(hObject, handles);

$\%$--- Executes during object creation, after setting all properties.

function max_seg4_type_7_CreateFcn(hObject, eventdata, handles)

$\%$ hObject handle to max_seg4_type_7 (see GCBO)

$\%$ eventdata reserved - to be defined in a future version of MATLAB

$\%$ handles empty - handles not created until after all CreateFcns called

\% Hint: edit controls usually have a white background on Windows. 


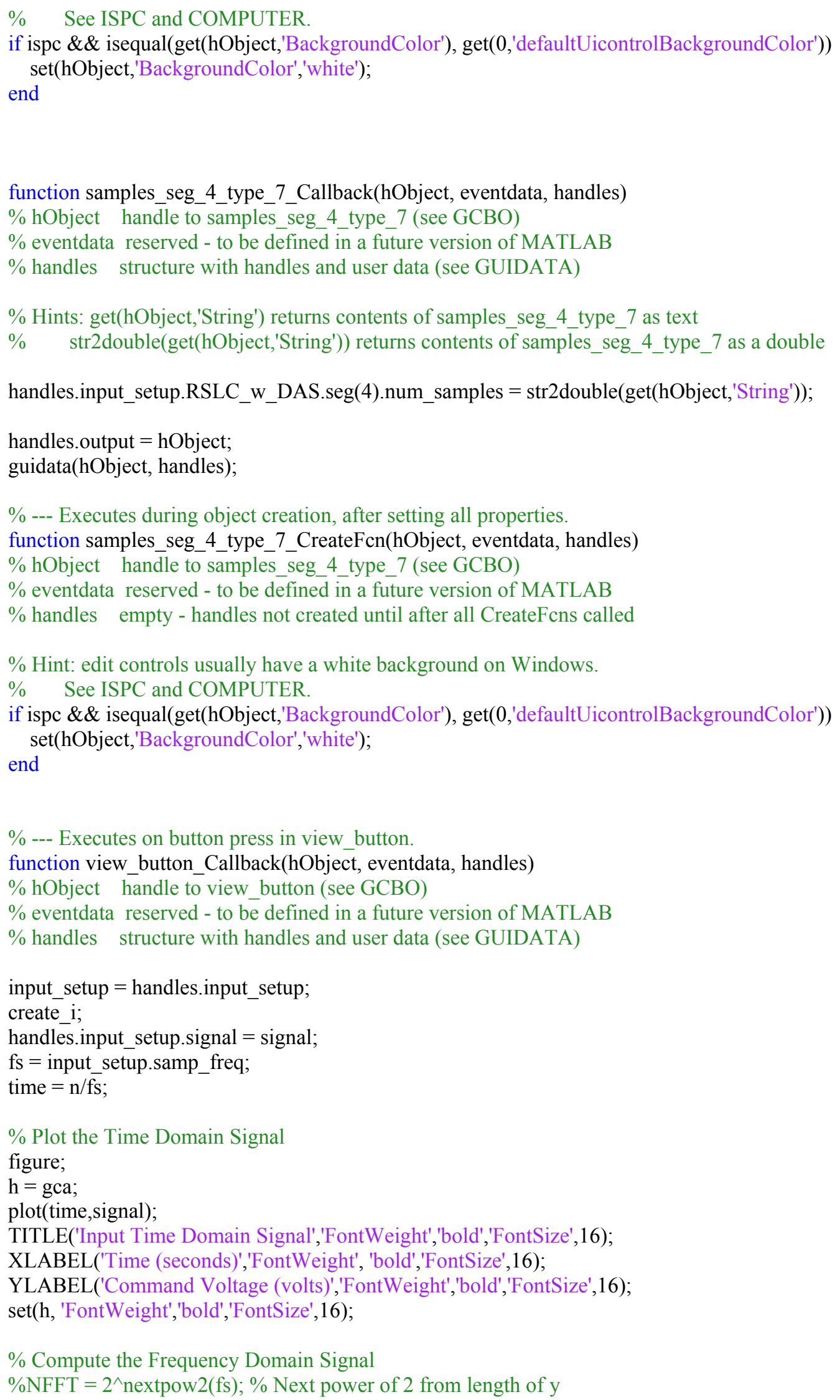




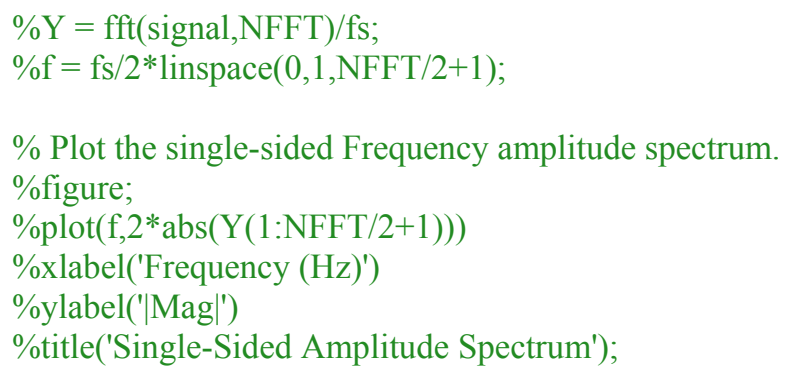

$\%$--- Executes when user attempts to close figure1.

function figure1_CloseRequestFcn(hObject, eventdata, handles)

$\%$ hObject handle to figurel (see GCBO)

$\%$ eventdata reserved - to be defined in a future version of MATLAB

$\%$ handles structure with handles and user data (see GUIDATA)

$\%$ input_setup $=$ handles.input_setup;

$\%$ create_i;

$\%$ handles.input_setup.signal = signal;

$\%$ input_setup $=$ handles.input_setup;

$\%$ save input_setup input_setup;

$\%$

$\%$ uiresume(handles.figure1); 


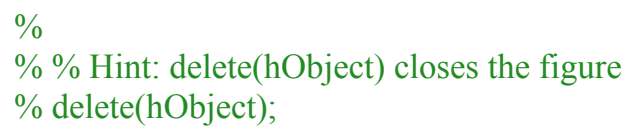

$\%$--- Executes on button press in "Save to Excel File".

function pushbutton7_Callback(hObject, eventdata, handles)

$\%$ hObject handle to pushbutton7 (see GCBO)

$\%$ eventdata reserved - to be defined in a future version of MATLAB

$\%$ handles structure with handles and user data (see GUIDATA)

input_setup $=$ handles.input_setup;

create_i; \% creates "signal"

[filename, pathname, filterindex] = uiputfile('*.xlsx', 'Save signal to Excel 2007 file');

$\mathrm{S}=\operatorname{strcat}($ pathname, filename);

xlswrite(S,signal'); 
function TotalStepTime_Callback(hObject, eventdata, handles)

$\%$ hObject handle to TotalStepTime (see GCBO)

$\%$ eventdata reserved - to be defined in a future version of MATLAB

$\%$ handles structure with handles and user data (see GUIDATA)

$\%$ Hints: get(hObject,'String') returns contents of TotalStepTime as text

$\% \quad$ str2double(get(hObject,'String')) returns contents of TotalStepTime as a double

handles.input_setup.StepFunction.TotalStepTime $=\operatorname{str} 2 \operatorname{double}($ get $($ hObject,'String' $))$;

handles.output $=$ hObject;

guidata(hObject, handles);

$\%$--- Executes during object creation, after setting all properties.

function TotalStepTime_CreateFcn(hObject, eventdata, handles)

$\%$ hObject handle to TotalStepTime (see GCBO)

$\%$ eventdata reserved - to be defined in a future version of MATLAB

$\%$ handles empty - handles not created until after all CreateFcns called

$\%$ Hint: edit controls usually have a white background on Windows.

$\% \quad$ See ISPC and COMPUTER.

if ispc \&\& isequal(get(hObject,'BackgroundColor'), get( 0 ,'defaultUicontrolBackgroundColor')) set(hObject,'BackgroundColor','white'); end

function TimeBeforeStep_Callback(hObject, eventdata, handles)

$\%$ hObject handle to TimeBeforeStep (see GCBO)

$\%$ eventdata reserved - to be defined in a future version of MATLAB

$\%$ handles structure with handles and user data (see GUIDATA)

$\%$ Hints: get(hObject,'String') returns contents of TimeBeforeStep as text

$\% \quad$ str2double(get(hObject,'String')) returns contents of TimeBeforeStep as a double

handles.input_setup.StepFunction.TimeBeforeStep = str2double $($ get $($ hObject,'String') $)$;

handles.output $=$ hObject;

guidata(hObject, handles);

$\%$--- Executes during object creation, after setting all properties.

function TimeBeforeStep_CreateFcn(hObject, eventdata, handles)

$\%$ hObject handle to TimeBeforeStep (see GCBO)

$\%$ eventdata reserved - to be defined in a future version of MATLAB

$\%$ handles empty - handles not created until after all CreateFcns called

$\%$ Hint: edit controls usually have a white background on Windows.

$\% \quad$ See ISPC and COMPUTER.

if ispc \&\& isequal(get(hObject,'BackgroundColor'), get(0,'defaultUicontrolBackgroundColor')) set(hObject,'BackgroundColor','white'); end 
function StepAmplitude_Callback(hObject, eventdata, handles)

$\%$ hObject handle to StepAmplitude (see GCBO)

$\%$ eventdata reserved - to be defined in a future version of MATLAB

$\%$ handles structure with handles and user data (see GUIDATA)

$\%$ Hints: get(hObject,'String') returns contents of StepAmplitude as text

$\% \quad$ str2double(get(hObject,'String')) returns contents of StepAmplitude as a double

handles.input_setup.StepFunction.StepAmplitude $=\operatorname{str} 2 \operatorname{double}($ get $($ hObject,'String' $))$;

handles.output $=$ hObject;

guidata(hObject, handles);

$\%$--- Executes during object creation, after setting all properties.

function StepAmplitude_CreateFcn(hObject, eventdata, handles)

$\%$ hObject handle to StepAmplitude (see GCBO)

$\%$ eventdata reserved - to be defined in a future version of MATLAB

$\%$ handles empty - handles not created until after all CreateFcns called

$\%$ Hint: edit controls usually have a white background on Windows.

$\% \quad$ See ISPC and COMPUTER.

if ispc \&\& isequal(get(hObject,'BackgroundColor'), get( 0 ,'defaultUicontrolBackgroundColor')) set(hObject,'BackgroundColor','white'); end 


\section{Update Signal Creation GUI:}

\section{File: update_GUI_Create_i.m}

\section{Description: Updates displayed values when loading previous settings.}

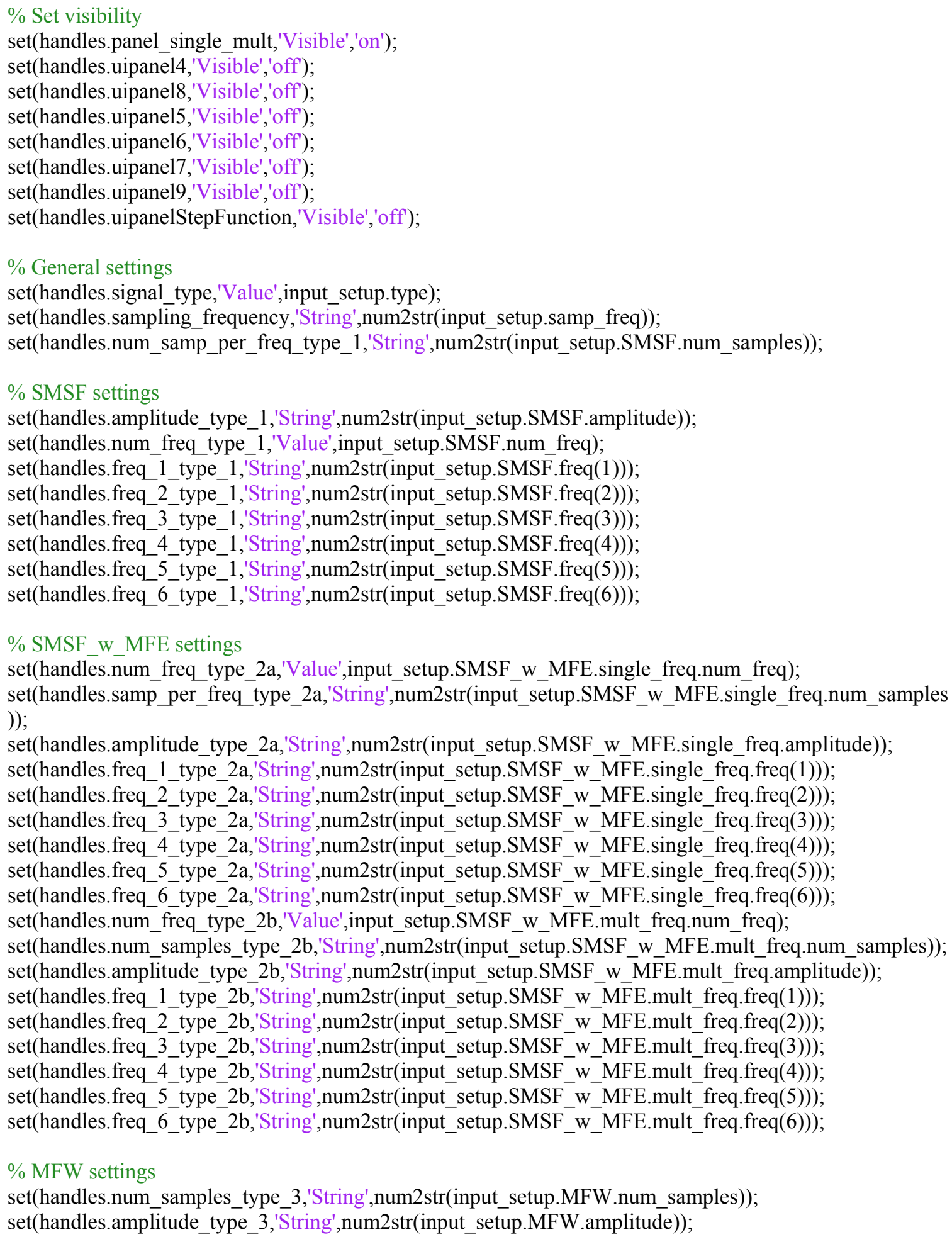


set(handles.num_freq_type_3,'Value',input_setup.MFW.num_freq);

set(handles.freq_1_type_3,'String',num2str(input_setup.MFW.freq(1)));

set(handles.freq_2_type_3,'String',num2str(input_setup.MFW.freq(2)));

set(handles.freq_3_type_3,'String',num2str(input_setup.MFW.freq(3)));

set(handles.freq 4 type 3 ,'String',num2str(input_setup.MFW.freq(4)));

set(handles.freq_5_type_3,'String',num2str(input_setup.MFW.freq(5)));

set(handles.freq_6_type_3,'String',num2str(input_setup.MFW.freq(6)));

$\%$ chirp settings

set(handles.amplitude_type_4,'String',num2str(input_setup.chirp.amplitude));

set(handles.time_type_4,'String',num2str(input_setup.chirp.time));

$\%$ NSCRS settings

set(handles.num_samples_type_5,'String',num2str(input_setup.NSCRS.num_samples)); set(handles.min_amplitude_type_5,'String',num2str(input_setup.NSCRS.min_amp)); set(handles.max_amplitude_type_5,'String',num2str(input_setup.NSCRS.max_amp)); set(handles.interval_type_5,'String',num2str(input_setup.NSCRS.interval));

$\%$ RSLC settings

set(handles.num_samples_type_6,'String',num2str(input_setup.RSLC.num_samples)); set(handles.min_amplitude_type_6,'String',num2str(input_setup.RSLC.min_amp)); set(handles.max_amplitude_type_6,'String',num2str(input_setup.RSLC.max_amp)); set(handles.min_interval_type_6,'String',num2str(input_setup.RSLC.min_interval)); set(handles.max_interval_type_6,'String',num2str(input_setup.RSLC.max_interval));

\%RSLC_W_DAS settings

set(handles.Segments_type_7,'Value',input_setup.RSLC_w_DAS.num_segments); set(handles.min_interval_type_7,'String',num2str(input_setup.RSLC_w_DAS.min_interval)); set(handles.max_interval_type_7,'String',num2str(input_setup.RSLC_w_DAS.max_interval)); set(handles.min_seg1_type_7,'String',num2str(input_setup.RSLC_w_DAS.seg(1).min_amp)); set(handles.min_seg2_type_7,'String',num2str(input_setup.RSLC_w_DAS.seg(2).min_amp)); set(handles.min_seg3_type_7,'String',num2str(input_setup.RSLC_w_DAS.seg(3).min_amp)); set(handles.min_seg4_type_7,'String',num2str(input_setup.RSLC_w_DAS.seg(4).min_amp)); set(handles.max_seg1_type_7,'String',num2str(input_setup.RSLC_w_DAS.seg(1).max_amp)); set(handles.max_seg2_type_7,'String',num2str(input_setup.RSLC_w_DAS.seg(2).max_amp)); set(handles.max_seg3_type_7,'String',num2str(input_setup.RSLC_w_DAS.seg(3).max_amp)); set(handles.max_seg4_type_7,'String',num2str(input_setup.RSLC_w_DAS.seg(4).max_amp)); set(handles.samples_seg_1_type_7,'String',num2str(input_setup.RSLC_w_DAS.seg(1).num_samples)); set(handles.samples_seg_2_type_7,'String',num2str(input_setup.RSLC_w_DAS.seg(2).num_samples)); set(handles.samples_seg_3_type_7,'String',num2str(input_setup.RSLC_w_DAS.seg(3).num_samples)); set(handles.samples_seg_4_type_7,'String',num2str(input_setup.RSLC_w_DAS.seg(4).num_samples));

$\%$ StepFunction settings set(handles.TotalStepTime,'String',num2str(input_setup.StepFunction.TotalStepTime)); set(handles.TimeBeforeStep,'Value',input_setup.StepFunction.TimeBeforeStep);

set(handles.StepAmplitude,'String',num2str(input_setup.StepFunction.StepAmplitude)); 


\section{Signal Creation Calculations:}

\section{File: create_i.m}

Description: Used in GUI_create_i.m file. This file holds the code to generate the timedomain data for the signals.

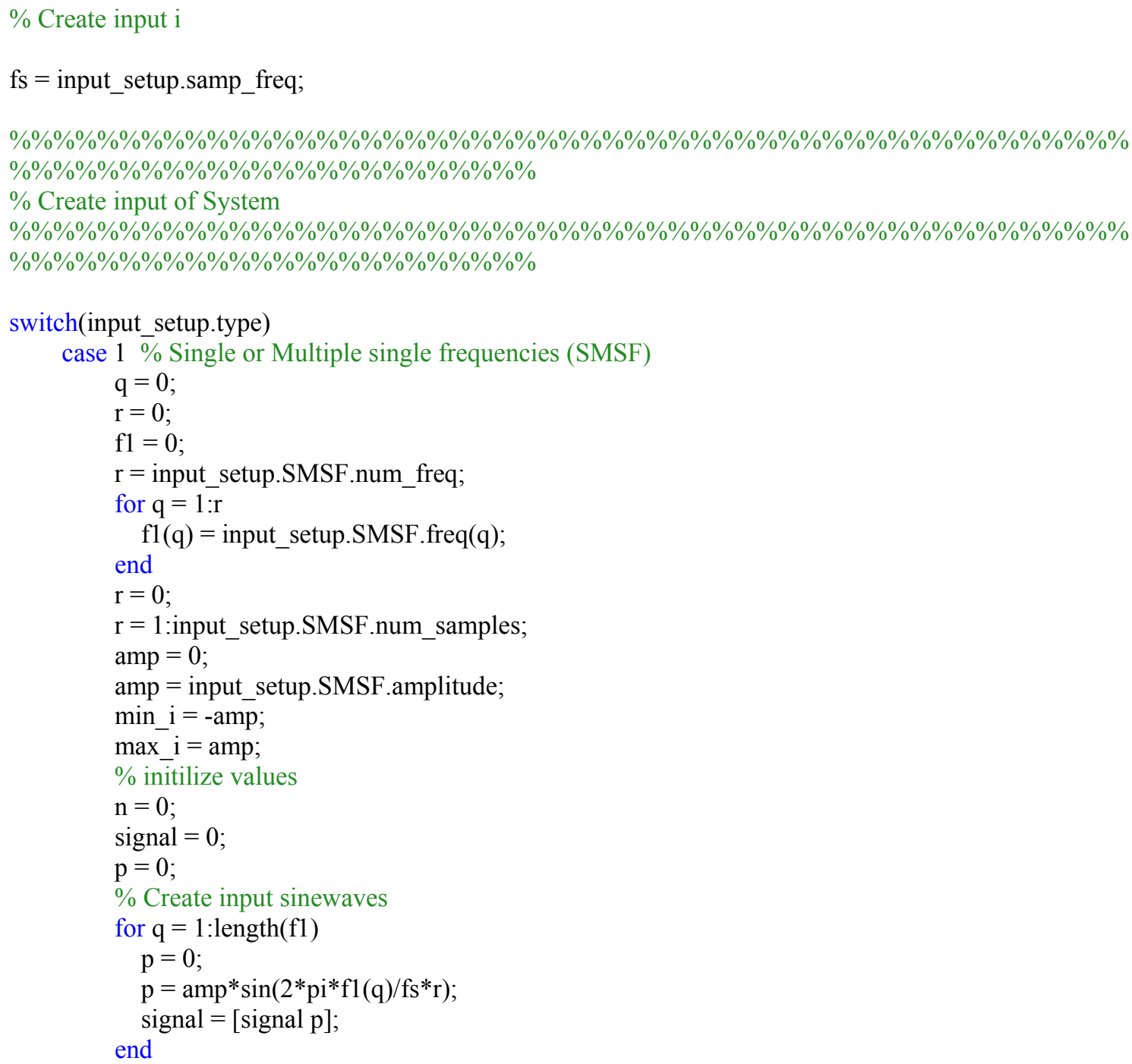

case $2 \%$ Single or Multiple single frequencies with Multiple frequency Wave attached at end (SMSF_w_MFE)

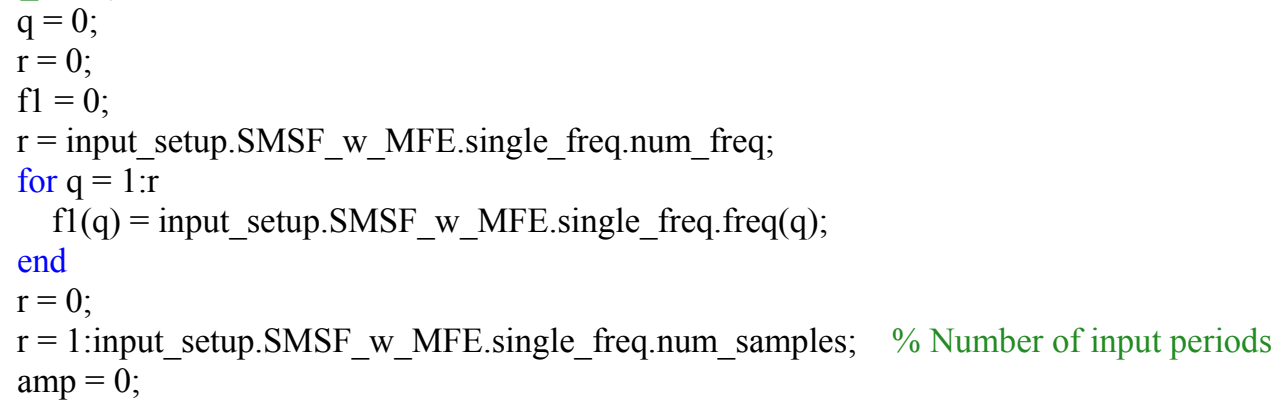




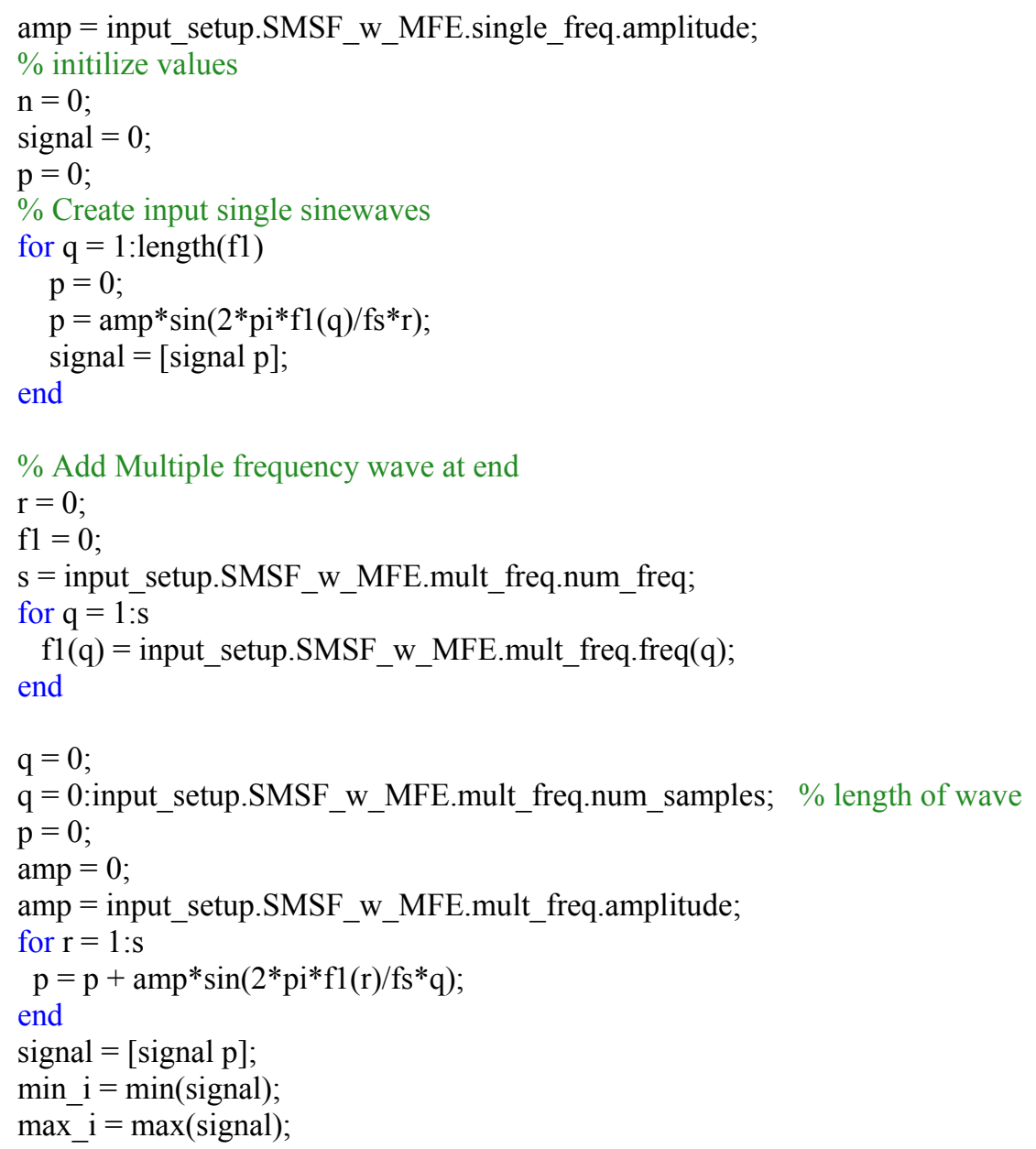




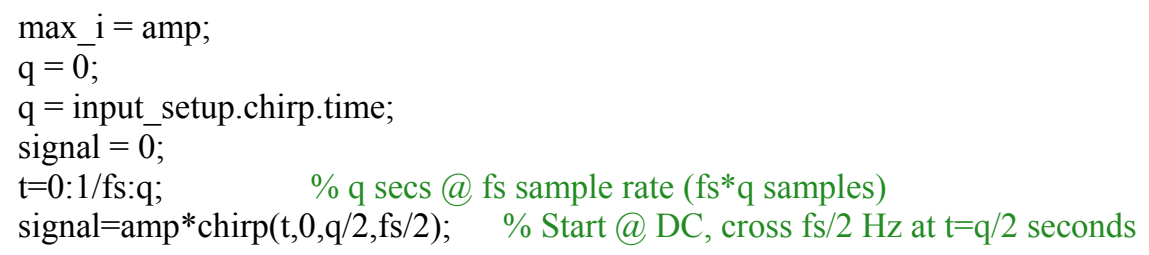

case $5 \%$ N-samples-Constant Random Signal (NSCRS)

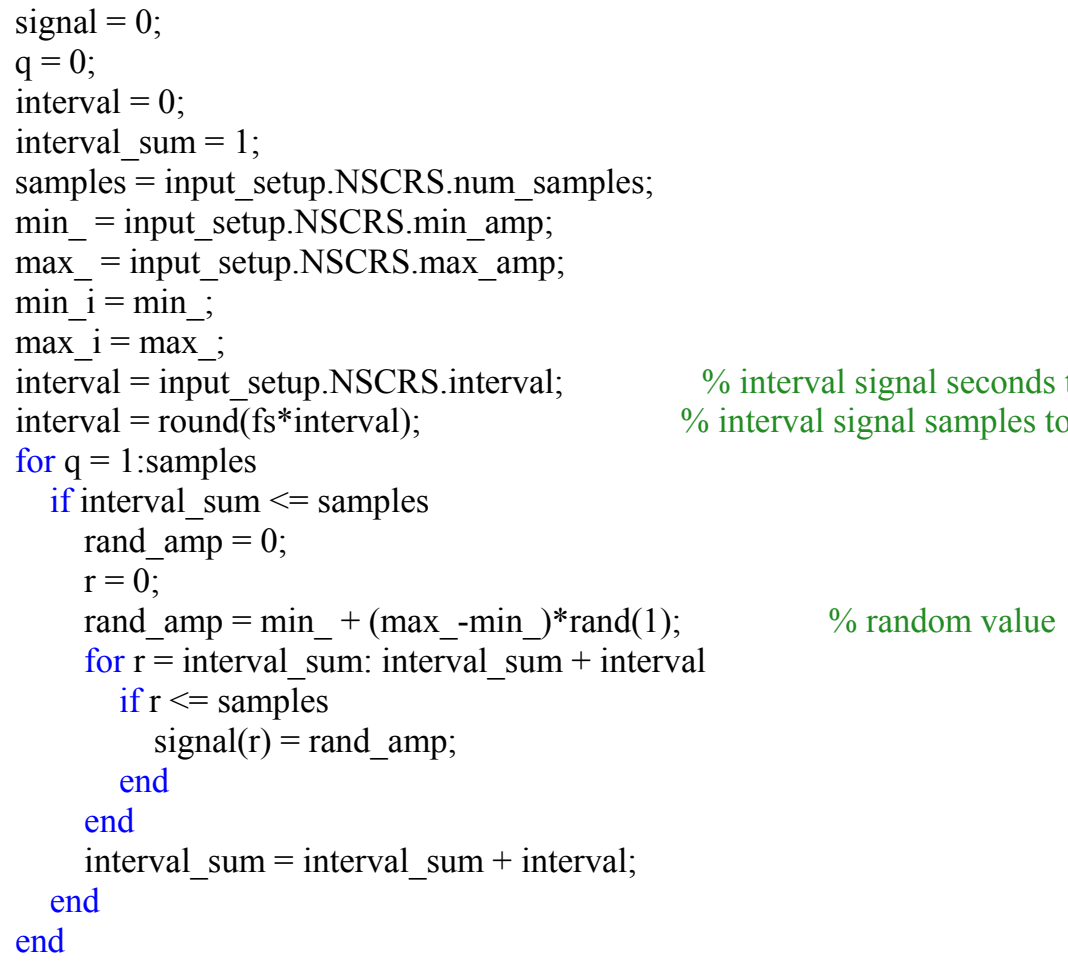

case $6 \%$ Random Signal with level change at random instances (RSLC)

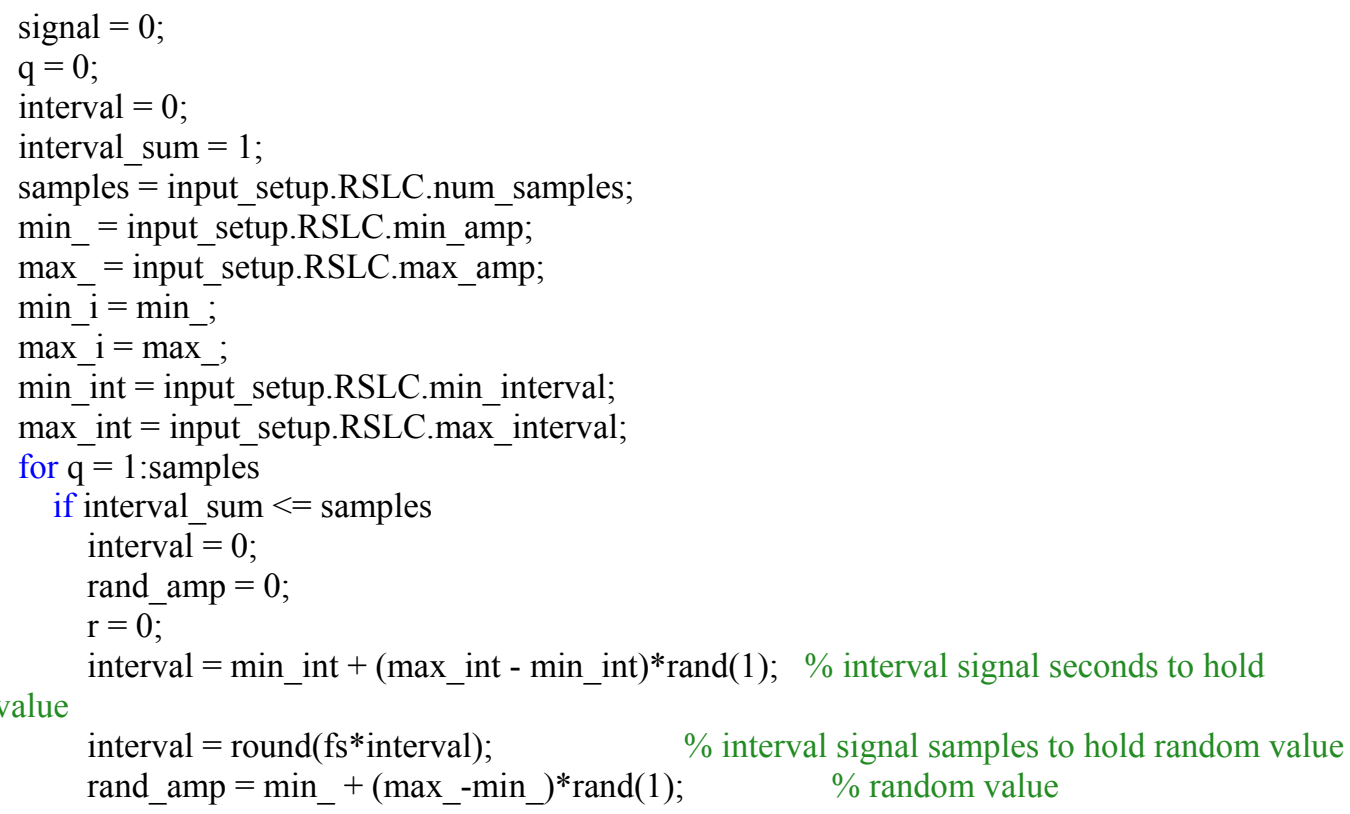




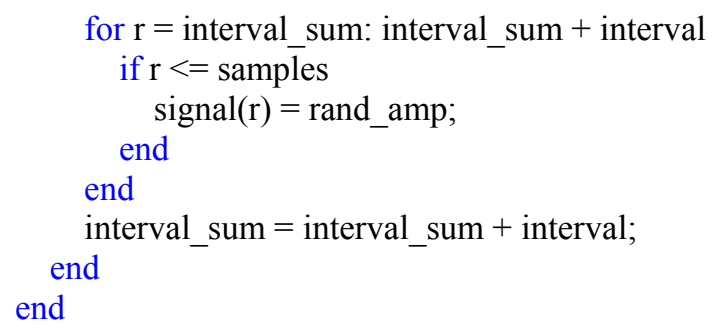

case $7 \%$ Random Signal with level change at random instances with multiple $\mathrm{min} / \mathrm{max}$ segments

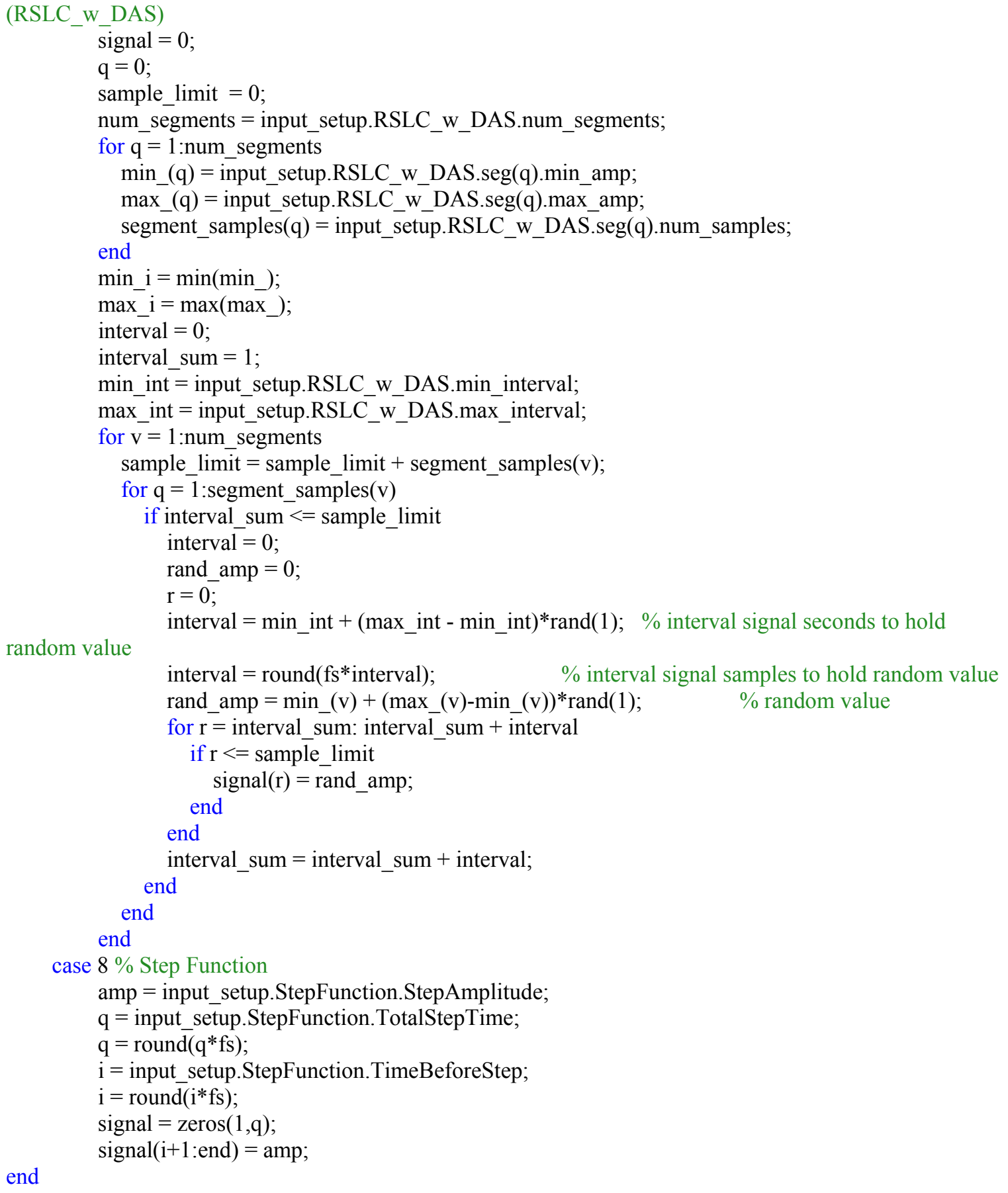




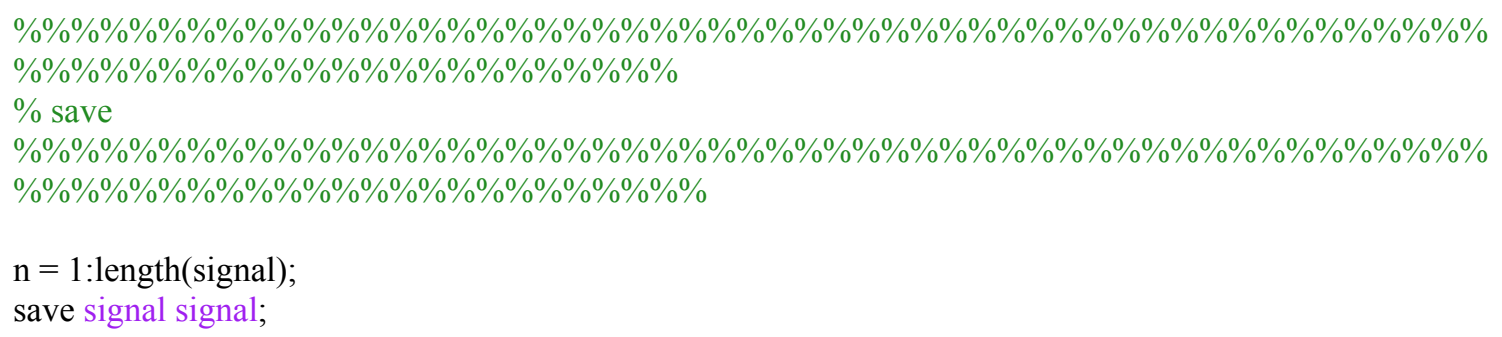




\section{Model Training GUI: Interface Used to Train \& Simulate Neural Network}

\section{File: GUI_model.m}

Description: Brings up a GUI interface which allows for creating a neural network, importing input/target data, plotting this data over time, plotting target vs. input, plotting input frequency spectrum and plotting plant frequency response. The GUI also allows for setup of Levenberg-Marquardt training parameters, training $\mathrm{NN}$ to input/target data, simulating network outputs, plotting simulation results, calculating mean squared error (mse) for simulation results vs target and error $\%(\mathrm{mse} /$ mean squared target value). The user can also re-initialize the $\mathrm{NN}$ weights at any time and can save or load neural network.

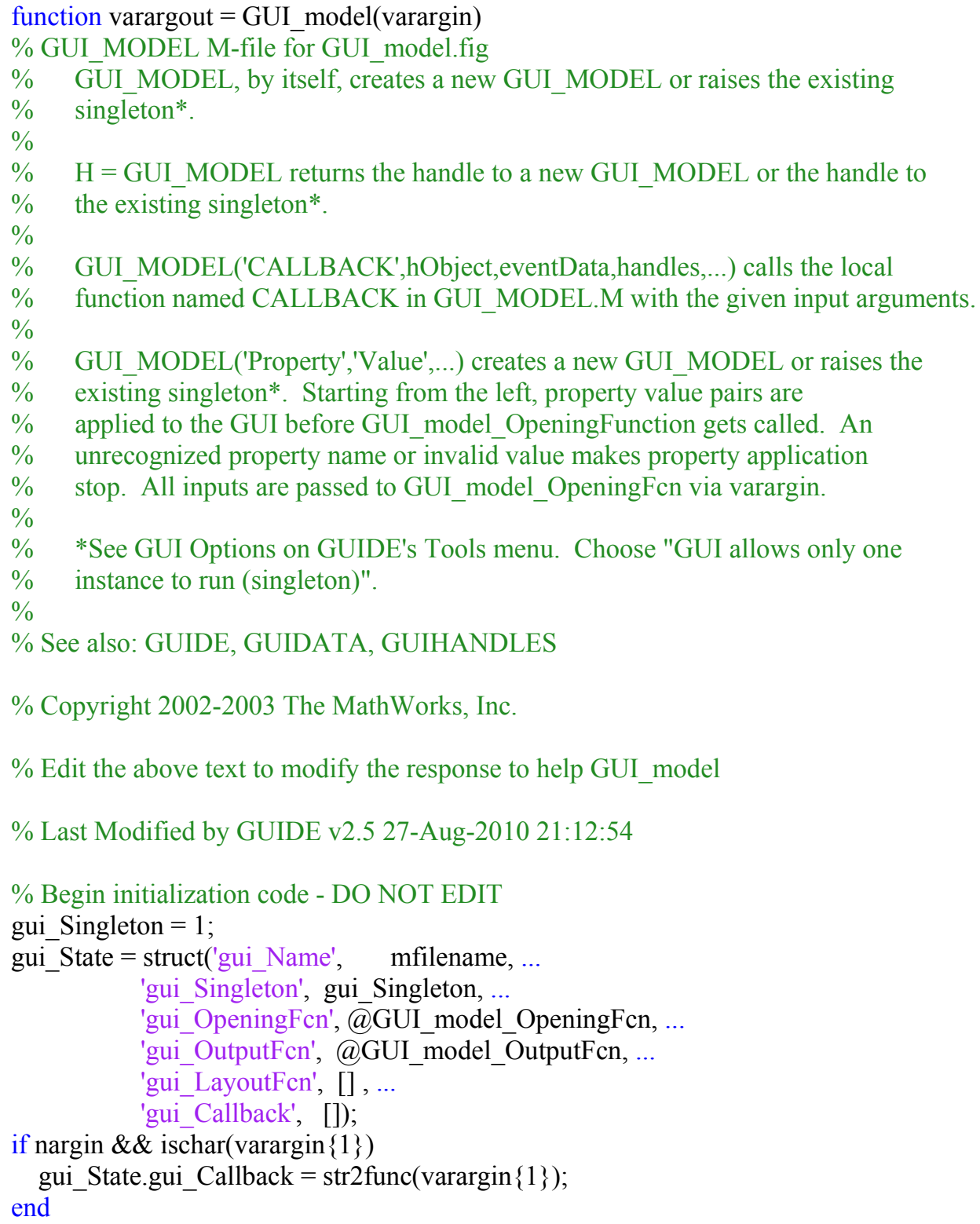




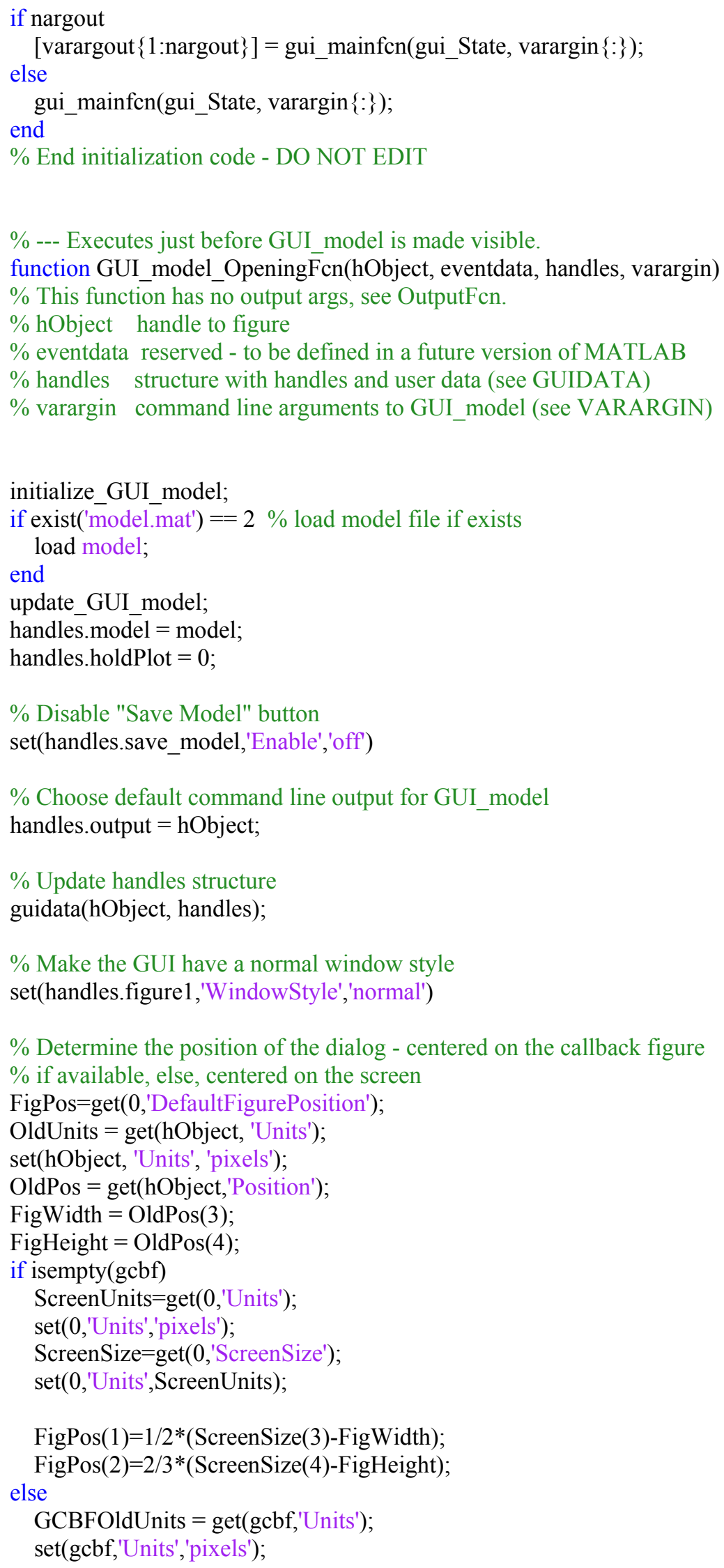




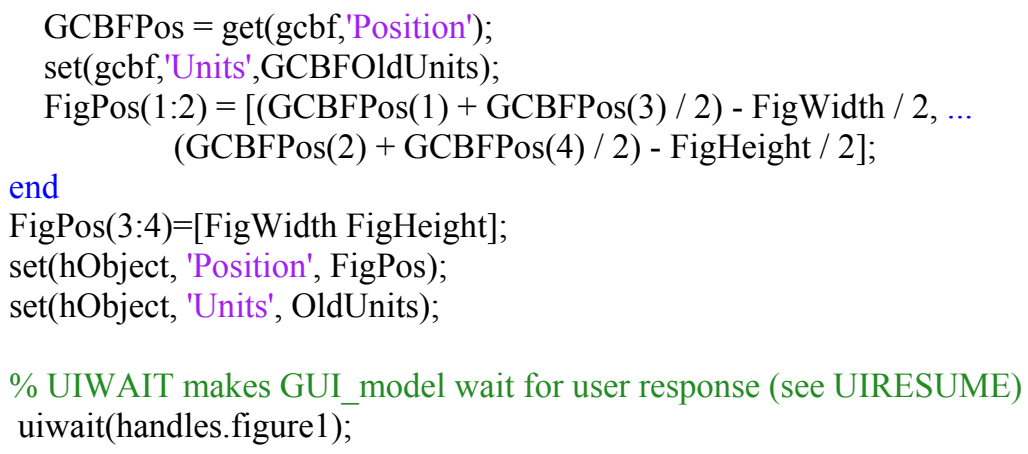




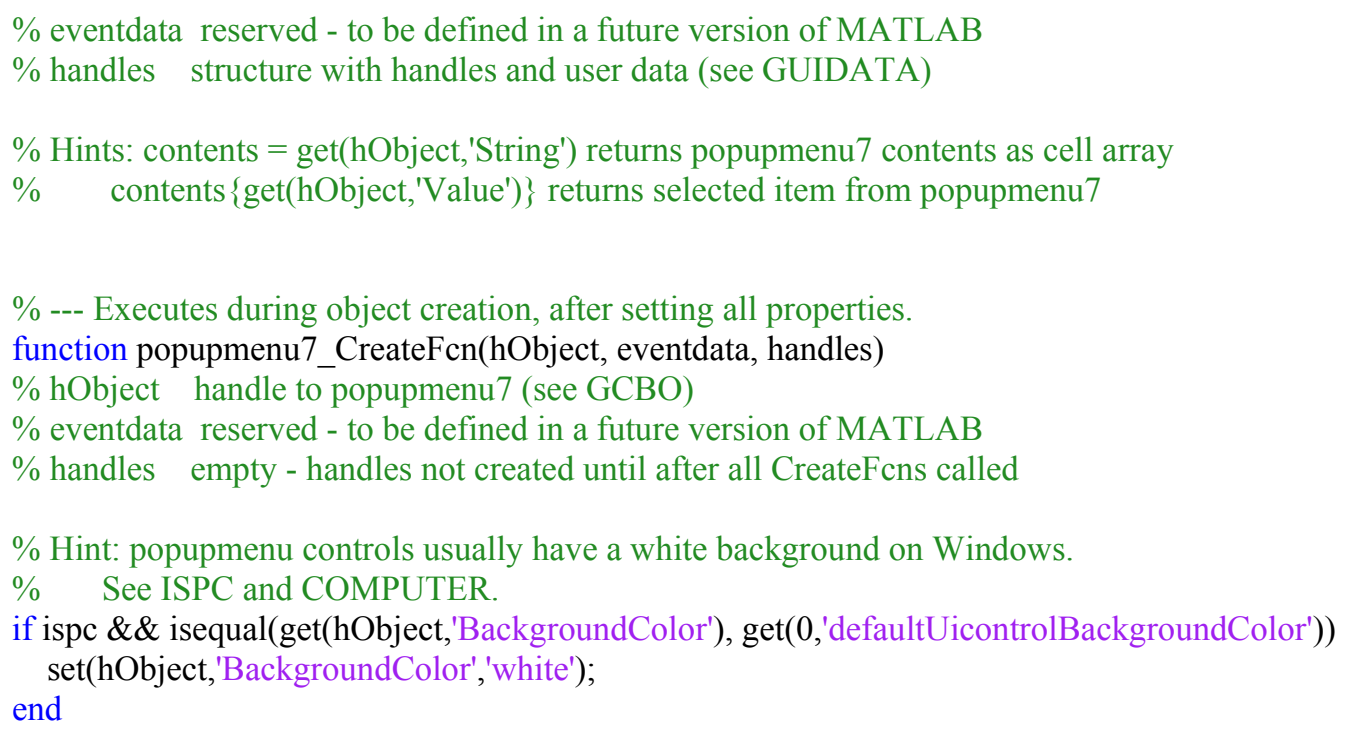

$\%$--- Executes during object creation, after setting all properties.

function model_rand_value_CreateFcn(hObject, eventdata, handles)

$\%$ hObject handle to model_rand_value (see GCBO)

$\%$ eventdata reserved - to be defined in a future version of MATLAB

$\%$ handles empty - handles not created until after all CreateFcns called

$\%$ Hint: edit controls usually have a white background on Windows.

$\% \quad$ See ISPC and COMPUTER.

if ispc \&\& isequal(get(hObject,'BackgroundColor'), get( 0, 'defaultUicontrolBackgroundColor')) set(hObject,'BackgroundColor','white'); end

function edit21_Callback(hObject, eventdata, handles)

$\%$ hObject handle to edit21 (see GCBO)

$\%$ eventdata reserved - to be defined in a future version of MATLAB

$\%$ handles structure with handles and user data (see GUIDATA)

$\%$ Hints: get(hObject,'String') returns contents of edit21 as text

$\% \quad$ str2double(get(hObject,'String')) returns contents of edit21 as a double 
$\%$--- Executes during object creation, after setting all properties.

function edit21_CreateFcn(hObject, eventdata, handles)

$\%$ hObject handle to edit21 (see GCBO)

$\%$ eventdata reserved - to be defined in a future version of MATLAB

$\%$ handles empty - handles not created until after all CreateFcns called

$\%$ Hint: edit controls usually have a white background on Windows.

$\% \quad$ See ISPC and COMPUTER.

if ispc \&\& isequal(get(hObject,'BackgroundColor'), get(0,'defaultUicontrolBackgroundColor')) set(hObject,'BackgroundColor','white'); end

function edit22_Callback(hObject, eventdata, handles)

$\%$ hObject handle to edit22 (see GCBO)

$\%$ eventdata reserved - to be defined in a future version of MATLAB

$\%$ handles structure with handles and user data (see GUIDATA)

$\%$ Hints: get(hObject,'String') returns contents of edit22 as text

$\% \quad$ str2double(get(hObject,'String')) returns contents of edit22 as a double

$\%$--- Executes during object creation, after setting all properties.

function edit22_CreateFcn(hObject, eventdata, handles)

$\%$ hObject handle to edit22 (see GCBO)

$\%$ eventdata reserved - to be defined in a future version of MATLAB

$\%$ handles empty - handles not created until after all CreateFcns called

$\%$ Hint: edit controls usually have a white background on Windows.

$\% \quad$ See ISPC and COMPUTER.

if ispc \&\& isequal(get(hObject,'BackgroundColor'), get( 0 ,'defaultUicontrolBackgroundColor')) set(hObject,'BackgroundColor','white');

end

function model_nw_ym_lower_Callback(hObject, eventdata, handles)

$\%$ hObject handle to model_nw ym_lower (see GCBO)

$\%$ eventdata reserved - to be defined in a future version of MATLAB

$\%$ handles structure with handles and user data (see GUIDATA)

$\%$ Hints: get(hObject,'String') returns contents of model_nw_ym_lower as text

$\% \quad$ str2double(get(hObject,'String')) returns contents of model__nw_ym_lower as a double

handles.model.structure.weight_init.nw.ym_lower = str2double(get(hObject,'String'));

handles.output $=$ hObject;

guidata(hObject, handles);

$\%$--- Executes during object creation, after setting all properties.

function model_nw_ym_lower_CreateFcn(hObject, eventdata, handles)

$\%$ hObject handle to model_nw_ym_lower (see GCBO)

$\%$ eventdata reserved - to be defined in a future version of MATLAB 
$\%$ handles empty - handles not created until after all CreateFcns called

$\%$ Hint: edit controls usually have a white background on Windows.

$\% \quad$ See ISPC and COMPUTER.

if ispc \&\& isequal(get(hObject,'BackgroundColor'), get( 0 ,'defaultUicontrolBackgroundColor')) set(hObject,'BackgroundColor','white'); end

function model_nw_u_upper_Callback(hObject, eventdata, handles)

$\%$ hObject handle to model_nw_u_upper (see GCBO)

$\%$ eventdata reserved - to be defined in a future version of MATLAB

$\%$ handles structure with handles and user data (see GUIDATA)

$\%$ Hints: get(hObject,'String') returns contents of model_nw_u_upper as text

$\% \quad$ str2double(get(hObject,'String')) returns contents of model_nw_u_upper as a double

handles.model.structure.weight_init.nw.u_upper =str2double(get(hObject,'String'));

handles.output $=$ hObject;

guidata(hObject, handles);

$\%$--- Executes during object creation, after setting all properties.

function model_nw_u_upper_CreateFcn(hObject, eventdata, handles)

$\%$ hObject handle to model_nw_u_upper (see GCBO)

$\%$ eventdata reserved - to be defined in a future version of MATLAB

$\%$ handles empty - handles not created until after all CreateFcns called

$\%$ Hint: edit controls usually have a white background on Windows.

$\% \quad$ See ISPC and COMPUTER.

if ispc \&\& isequal(get(hObject,'BackgroundColor'), get(0,'defaultUicontrolBackgroundColor')) set(hObject,'BackgroundColor','white'); end

function model_nw_yp_upper_Callback(hObject, eventdata, handles)

$\%$ hObject handle to model_nw_yp_upper (see GCBO)

$\%$ eventdata reserved - to be defined in a future version of MATLAB

$\%$ handles structure with handles and user data (see GUIDATA)

\% Hints: get(hObject,'String') returns contents of model_nw_yp_upper as text

$\% \quad$ str2double(get(hObject,'String')) returns contents of model_nw_yp_upper as a double

handles.model.structure.weight_init.nw.yp_upper $=$ str2double $($ get $($ hObject,'String' $))$;

handles.output $=$ hObject;

guidata(hObject, handles);

$\%$--- Executes during object creation, after setting all properties.

function model_nw_yp_upper_CreateFcn(hObject, eventdata, handles)

$\%$ hObject handle to model_nw_yp_upper (see GCBO)

$\%$ eventdata reserved - to be defined in a future version of MATLAB

$\%$ handles empty - handles not created until after all CreateFcns called 
$\%$ Hint: edit controls usually have a white background on Windows.

$\% \quad$ See ISPC and COMPUTER.

if ispc \&\& isequal(get(hObject,'BackgroundColor'), get(0,'defaultUicontrolBackgroundColor')) set(hObject,'BackgroundColor','white');

end

function model_nw_ym_upper_Callback(hObject, eventdata, handles)

$\%$ hObject handle to model_nw_ym_upper (see GCBO)

$\%$ eventdata reserved - to be defined in a future version of MATLAB

$\%$ handles structure with handles and user data (see GUIDATA)

$\%$ Hints: get(hObject,'String') returns contents of model_nw_ym_upper as text

$\% \quad$ str2double(get(hObject,'String')) returns contents of model nw_ym upper as a double

handles.model.structure.weight_init.nw.ym_upper = str2double(get(hObject,'String'));

handles.output $=$ hObject;

guidata(hObject, handles);

$\%$--- Executes during object creation, after setting all properties.

function model_nw_ym_upper_CreateFcn(hObject, eventdata, handles)

$\%$ hObject handle to model_nw_ym_upper(see GCBO)

$\%$ eventdata reserved - to be defined in a future version of MATLAB

$\%$ handles empty - handles not created until after all CreateFcns called

$\%$ Hint: edit controls usually have a white background on Windows.

$\% \quad$ See ISPC and COMPUTER.

if ispc \&\& isequal(get(hObject,'BackgroundColor'), get(0,'defaultUicontrolBackgroundColor')) set(hObject,'BackgroundColor','white'); end

$\%$--- Executes on button press in checkbox 5 .

function checkbox5_Callback(hObject, eventdata, handles)

$\%$ hObject handle to checkbox5 (see GCBO)

$\%$ eventdata reserved - to be defined in a future version of MATLAB

$\%$ handles structure with handles and user data (see GUIDATA)

\% Hint: get(hObject,'Value') returns toggle state of checkbox 5

function edit27_Callback(hObject, eventdata, handles)

$\%$ hObject handle to edit27 (see GCBO)

$\%$ eventdata reserved - to be defined in a future version of MATLAB

$\%$ handles structure with handles and user data (see GUIDATA)

$\%$ Hints: get(hObject,'String') returns contents of edit27 as text

$\% \quad$ str2double(get(hObject,'String')) returns contents of edit27 as a double

$\%$--- Executes during object creation, after setting all properties.

function edit27_CreateFcn(hObject, eventdata, handles)

$\%$ hObject handle to edit27 (see GCBO) 


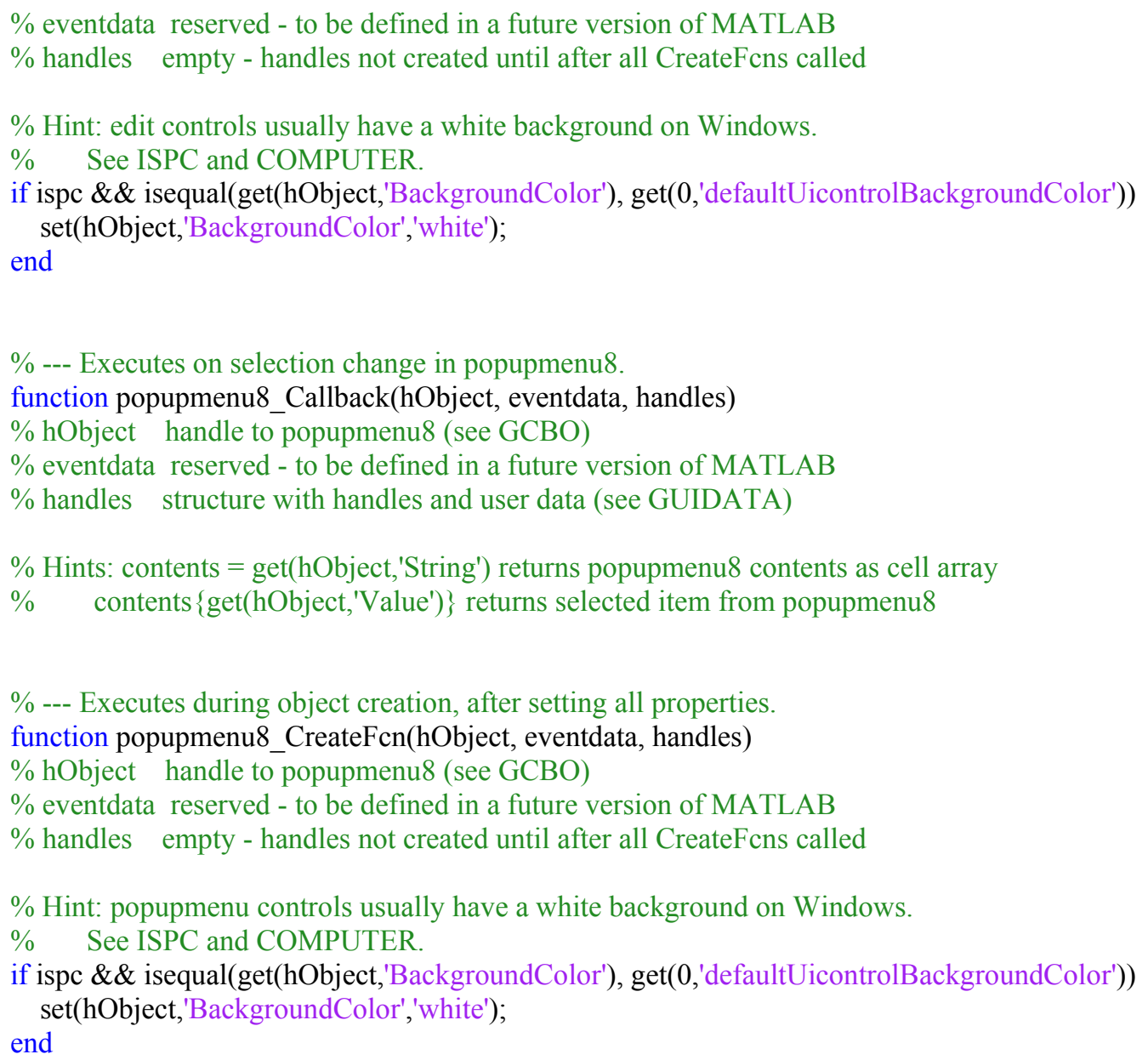




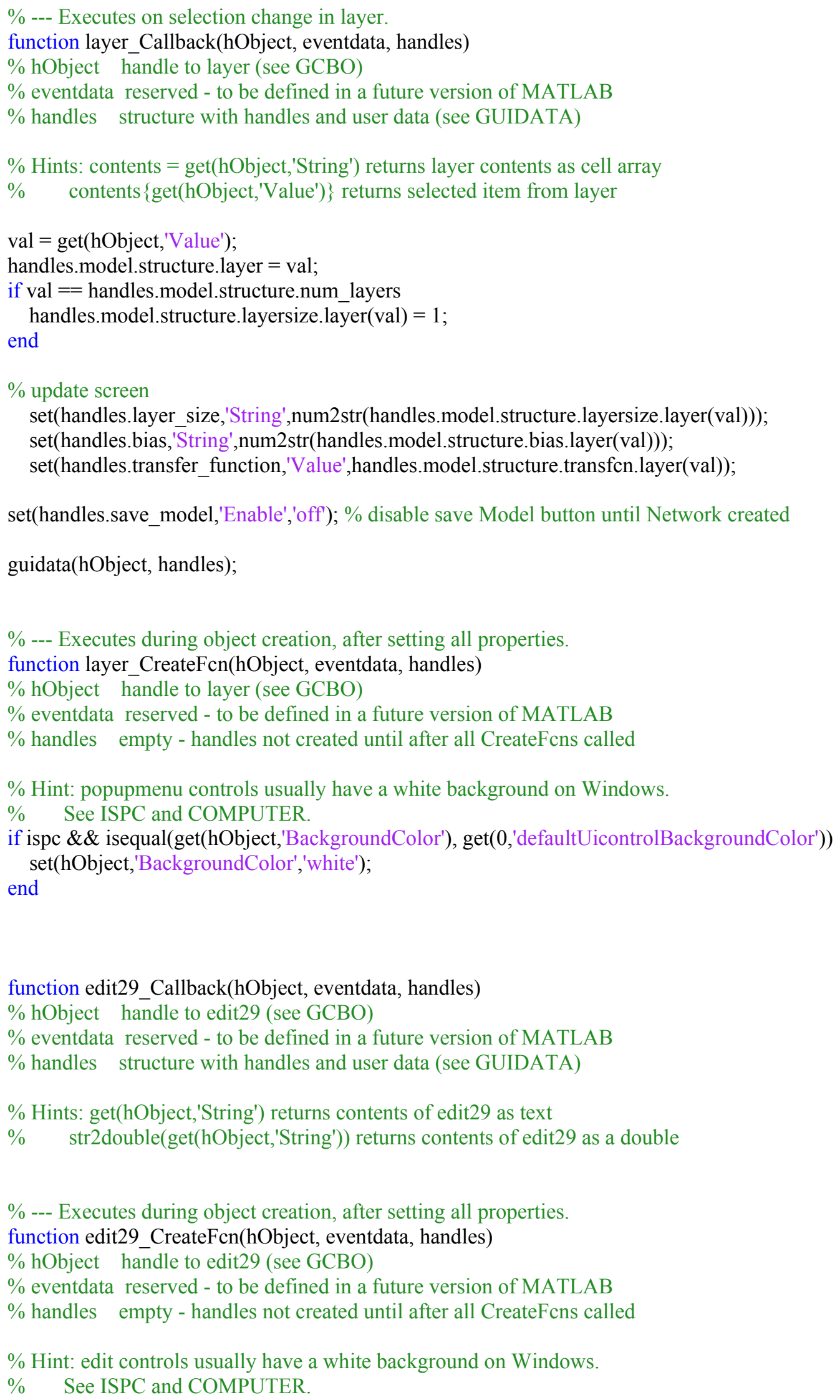


if ispc \&\& isequal(get(hObject,'BackgroundColor'), get( 0 ,'defaultUicontrolBackgroundColor')) set(hObject,'BackgroundColor','white');

end

$\%$--- Executes on button press in pushbutton6.

function pushbutton6_Callback(hObject, eventdata, handles)

$\%$ hObject handle to pushbutton6 (see GCBO)

$\%$ eventdata reserved - to be defined in a future version of MATLAB

$\%$ handles structure with handles and user data (see GUIDATA)

$\%$--- Executes on button press in pushbutton7.

function pushbutton7_Callback(hObject, eventdata, handles)

$\%$ hObject handle to pushbutton7 (see GCBO)

$\%$ eventdata reserved - to be defined in a future version of MATLAB

$\%$ handles structure with handles and user data (see GUIDATA)

$\%$--- Executes on button press in checkbox6.

function checkbox6_Callback(hObject, eventdata, handles)

$\%$ hObject handle to checkbox6 (see GCBO)

$\%$ eventdata reserved - to be defined in a future version of MATLAB

$\%$ handles structure with handles and user data (see GUIDATA)

\% Hint: get(hObject,'Value') returns toggle state of checkbox6

function min_u_Callback(hObject, eventdata, handles)

$\%$ hObject handle to min_u (see GCBO)

$\%$ eventdata reserved - to be defined in a future version of MATLAB

$\%$ handles structure with handles and user data (see GUIDATA)

$\%$ Hints: get(hObject,'String') returns contents of min_u as text

$\% \quad$ str2double(get(hObject,'String')) returns contents of min_u as a double

handles.model.structure.input_type.scaling.u_min = str2double(get(hObject,'String'));

handles.output $=$ hObject;

guidata(hObject, handles);

$\%$--- Executes during object creation, after setting all properties.

function min_u_CreateFcn(hObject, eventdata, handles)

$\%$ hObject handle to min_u (see GCBO)

$\%$ eventdata reserved - to be defined in a future version of MATLAB

$\%$ handles empty - handles not created until after all CreateFcns called

$\%$ Hint: edit controls usually have a white background on Windows.

$\% \quad$ See ISPC and COMPUTER.

if ispc \&\& isequal(get(hObject,'BackgroundColor'), get( 0, 'defaultUicontrolBackgroundColor')) set(hObject,'BackgroundColor','white'); end 


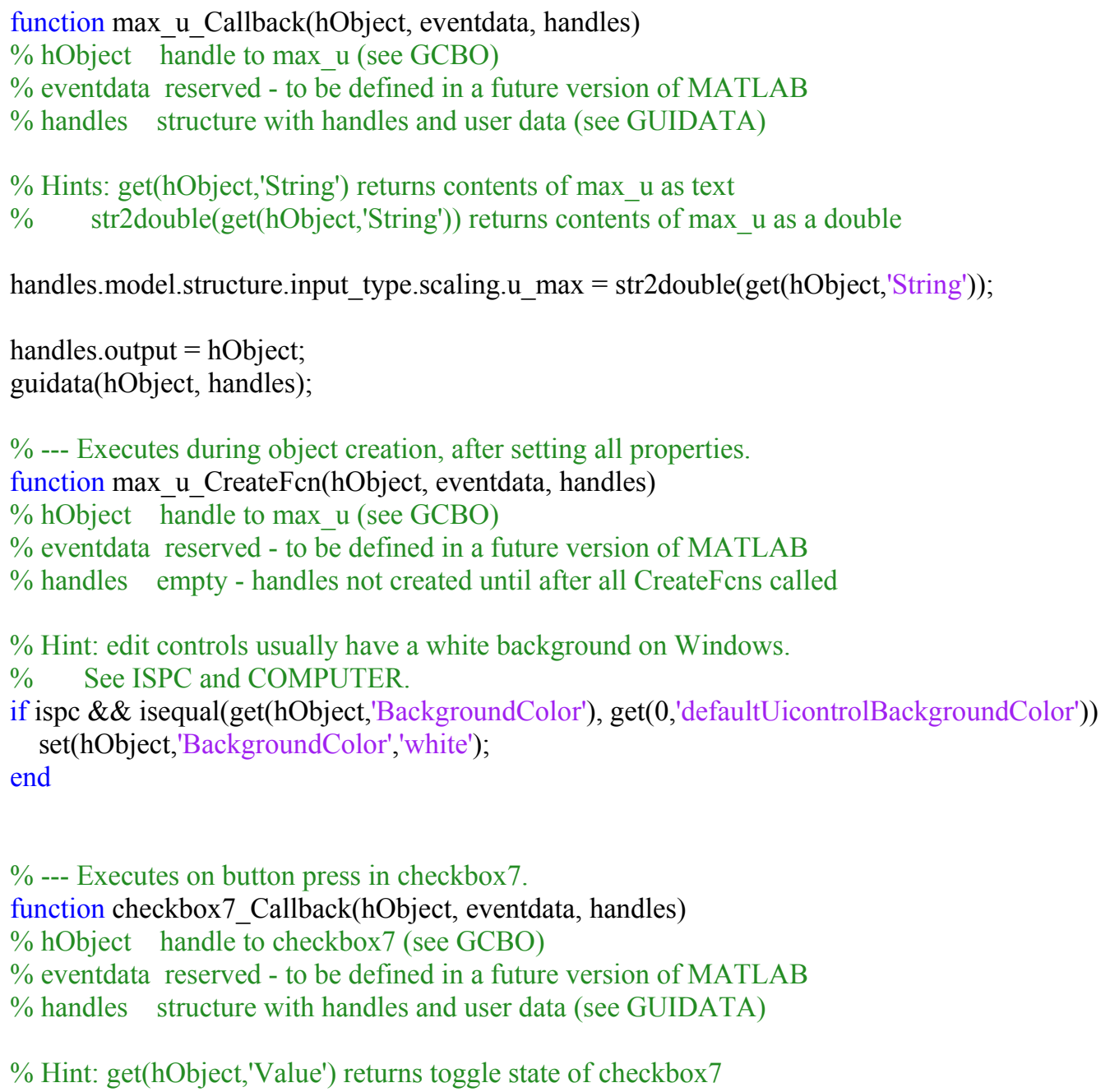

function min_yp_Callback(hObject, eventdata, handles)

$\%$ hObject handle to min_yp (see GCBO)

$\%$ eventdata reserved - to be defined in a future version of MATLAB

$\%$ handles structure with handles and user data (see GUIDATA)

$\%$ Hints: get(hObject,'String') returns contents of min_yp as text

$\% \quad$ str2double(get(hObject,'String')) returns contents of min_yp as a double

handles.model.structure.input_type.scaling.yp_min $=\operatorname{str} 2 \operatorname{double}($ get $($ hObject,'String' $))$;

handles.output $=$ hObject;

guidata(hObject, handles);

$\%$--- Executes during object creation, after setting all properties.

function min_yp_CreateFcn(hObject, eventdata, handles)

$\%$ hObject handle to min_yp (see GCBO)

$\%$ eventdata reserved - to be defined in a future version of MATLAB

$\%$ handles empty - handles not created until after all CreateFcns called 


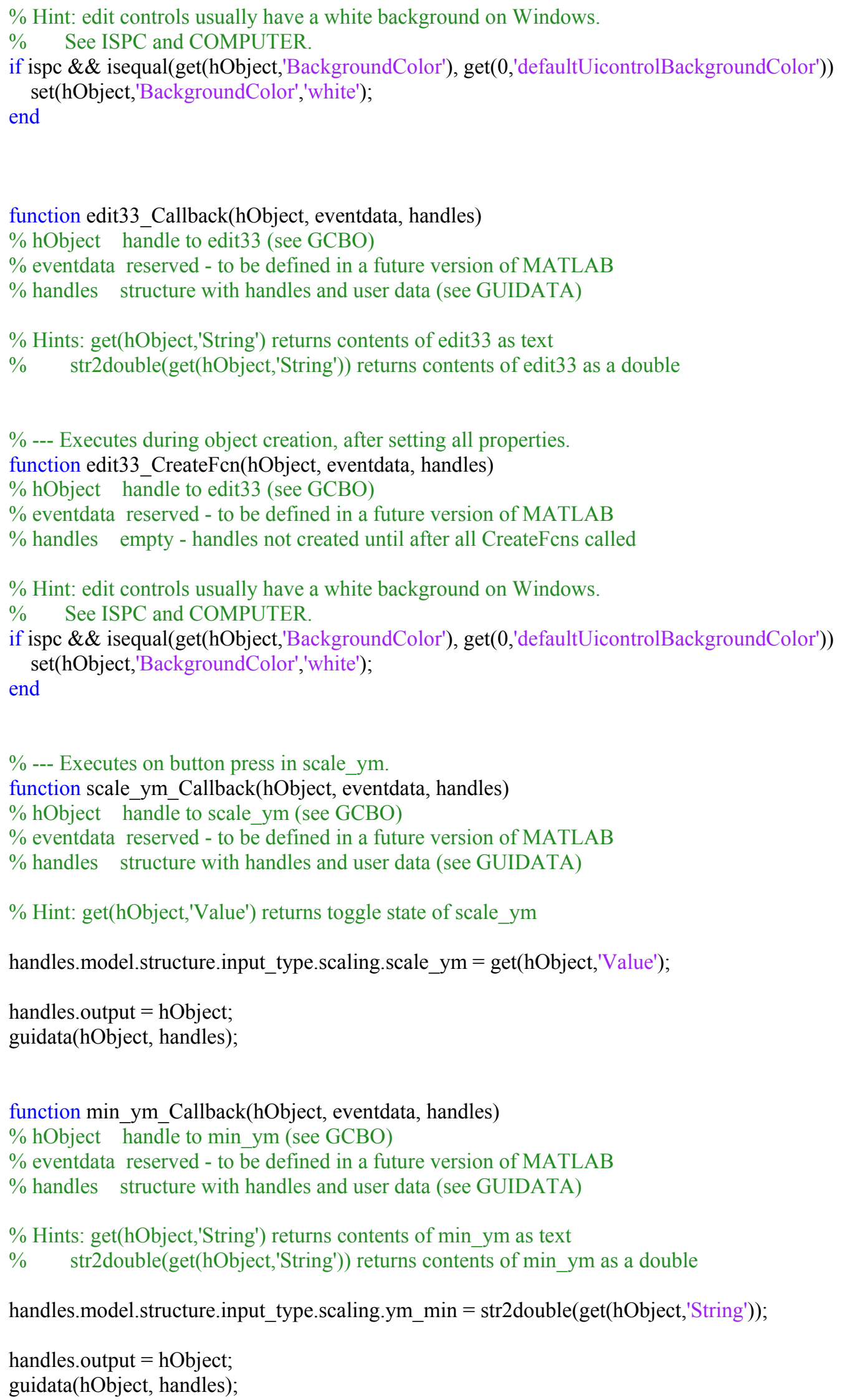




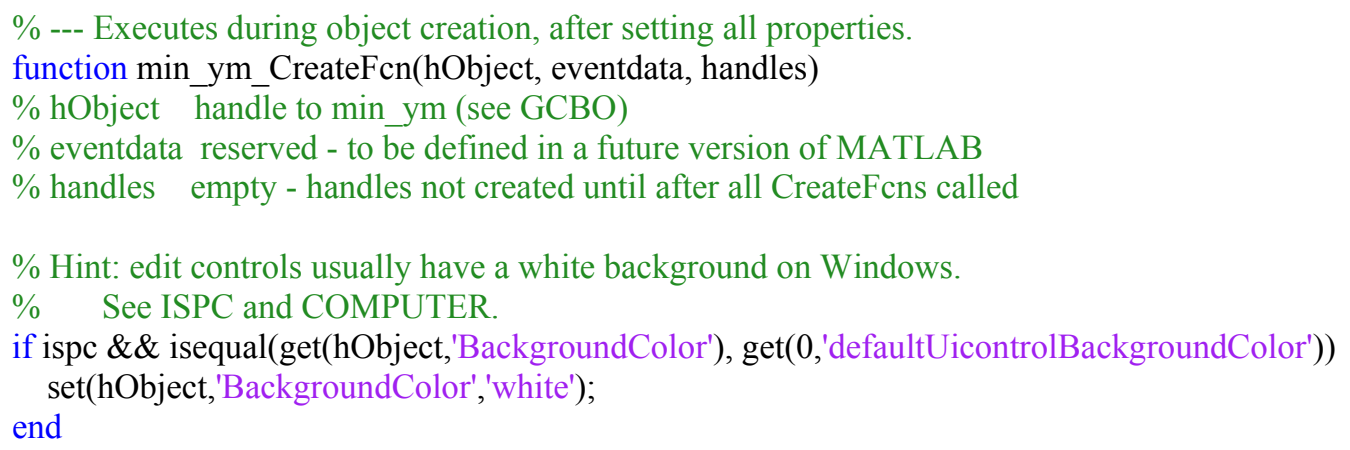


set(hObject,'BackgroundColor','white'); end

function edit36_Callback(hObject, eventdata, handles)

$\%$ hObject handle to edit36 (see GCBO)

$\%$ eventdata reserved - to be defined in a future version of MATLAB

$\%$ handles structure with handles and user data (see GUIDATA)

$\%$ Hints: get(hObject,'String') returns contents of edit36 as text

$\% \quad$ str2double(get(hObject,'String')) returns contents of edit36 as a double

$\%$--- Executes during object creation, after setting all properties.

function edit36_CreateFcn(hObject, eventdata, handles)

$\%$ hObject handle to edit36 (see GCBO)

$\%$ eventdata reserved - to be defined in a future version of MATLAB

$\%$ handles empty - handles not created until after all CreateFcns called

$\%$ Hint: edit controls usually have a white background on Windows.

$\% \quad$ See ISPC and COMPUTER.

if ispc \&\& isequal(get(hObject,'BackgroundColor'), get( 0 ,'defaultUicontrolBackgroundColor')) set(hObject,'BackgroundColor','white'); end

function edit37_Callback(hObject, eventdata, handles)

$\%$ hObject handle to edit37 (see GCBO)

$\%$ eventdata reserved - to be defined in a future version of MATLAB

$\%$ handles structure with handles and user data (see GUIDATA)

$\%$ Hints: get(hObject,'String') returns contents of edit37 as text

$\% \quad$ str2double(get(hObject,'String')) returns contents of edit37 as a double

$\%$--- Executes during object creation, after setting all properties.

function edit37_CreateFcn(hObject, eventdata, handles)

$\%$ hObject handle to edit37 (see GCBO)

$\%$ eventdata reserved - to be defined in a future version of MATLAB

$\%$ handles empty - handles not created until after all CreateFcns called

$\%$ Hint: edit controls usually have a white background on Windows.

$\% \quad$ See ISPC and COMPUTER.

if ispc \&\& isequal(get(hObject,'BackgroundColor'), get(0,'defaultUicontrolBackgroundColor')) set(hObject,'BackgroundColor','white');

end

function edit38_Callback(hObject, eventdata, handles)

$\%$ hObject handle to edit38 (see GCBO)

$\%$ eventdata reserved - to be defined in a future version of MATLAB

$\%$ handles structure with handles and user data (see GUIDATA) 


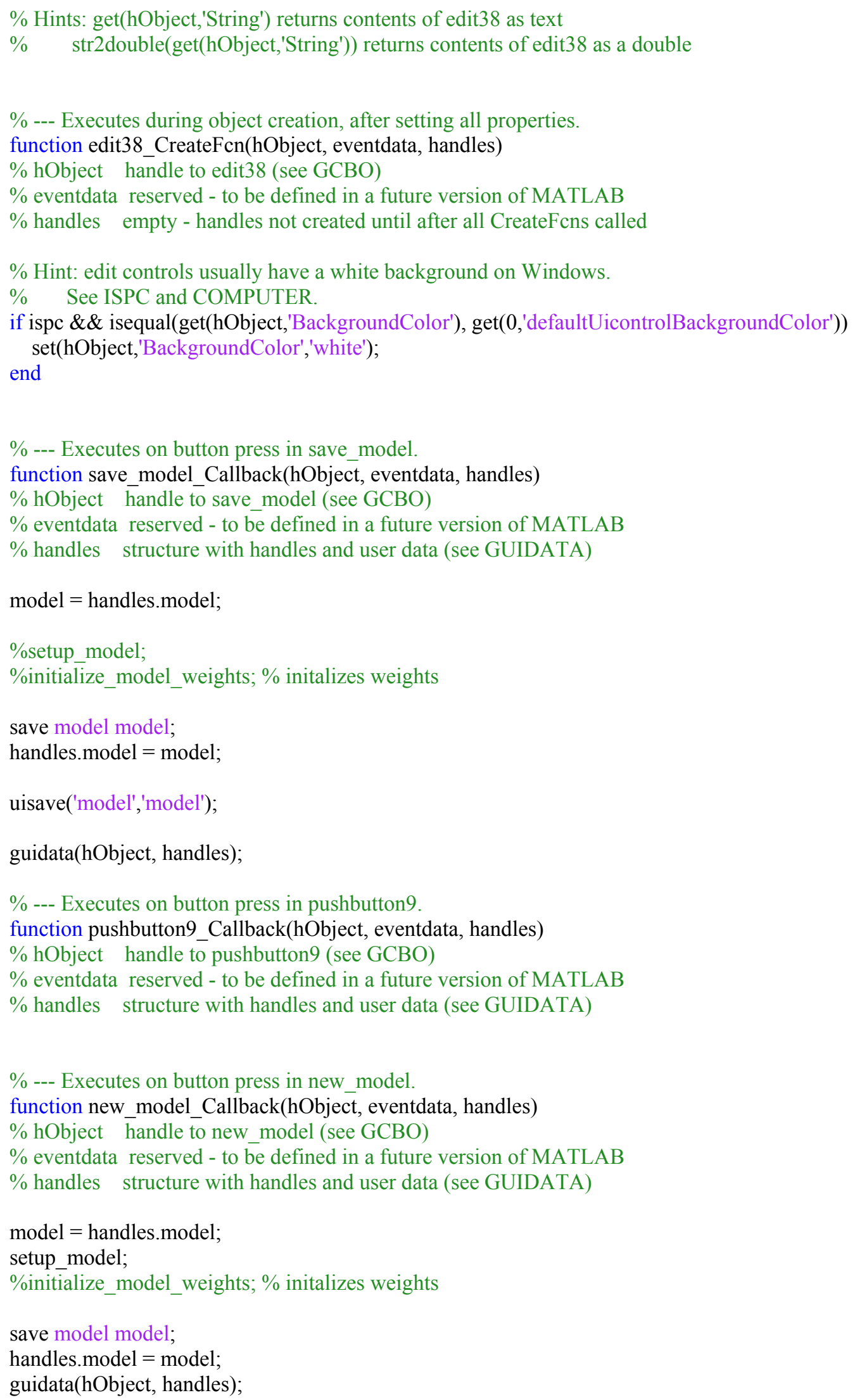


$\%$--- Executes on button press in load_model_NN.

function load_model_NN_Callback(hÖbject, eventdata, handles)

$\%$ hObject handle to load model NN (see GCBO)

$\%$ eventdata reserved - to be defined in a future version of MATLAB

$\%$ handles structure with handles and user data (see GUIDATA)

[filename, pathname, filterindex] = uigetfile('*.mat', 'Pick your model file');

load(filename);

update_GUI_model; \% shows loaded settings on screen

handles.model $=$ model;

save model model;

guidata(hObject, handles);

function num_layers_Callback(hObject, eventdata, handles)

$\%$ hObject handle to num_layers (see GCBO)

$\%$ eventdata reserved - to be defined in a future version of MATLAB

$\%$ handles structure with handles and user data (see GUIDATA)

$\%$ Hints: get(hObject,'String') returns contents of num_layers as text

$\% \quad$ str2double(get(hObject,'String')) returns contents of num_layers as a double

val $=\operatorname{str} 2$ double $\left(\right.$ get $\left(\right.$ hObject, ${ }^{\prime}$ String' $\left.)\right) ; \%$ val $=$ number of layers in the network

$\%$ limit number of layers $>=2$

if val $<=1$

set(handles.num_layers,'String','2');

end

$\%$ Store number of layers and setup layer pull down menu

set(handles.layer,'Value',1);

handles.model.structure.num_layers $=$ val;

val2 $=\{\}$;

for $\mathrm{r}=1$ : val

$\operatorname{val} 2\{\mathrm{r}, 1\}=$ num $2 \operatorname{str}(\mathrm{r})$;

end

set(handles.layer,'String',val2);

$\%$ Set layer size, bias, and transfer function if not already done (for each layer)

for $\mathrm{r}=1$ :val

if $\mathrm{r}<=$ length(handles.model.structure.layersize.layer) $\%$ test if value already stored

set(handles.layer_size,'String',num2str(handles.model.structure.layersize.layer(r))); else

handles.model.structure.layersize.layer $(\mathrm{r})=1 ; \%$ default $=1$

end

if $\mathrm{r}<=$ length(handles.model.structure.transfen.layer)

set(handles.transfer_function,'Value',handles.model.structure.transfen.layer(r)); else

handles.model.structure.transfcn.layer(r) $=1 ; \%$ default $=1$

end

if $\mathrm{r}<=$ length(handles.model.structure.bias.layer)

set(handles.bias, 'String',num2str(handles.model.structure.bias.layer(r))); else

handles.model.structure.bias.layer(r) $=1 ; \%$ default $=1$ 
end
end

set(handles.save_model,'Enable','off'); \% disable save Model button until Network created

handles.model.structure.layersize.layer(val) $=1$;

guidata(hObject, handles);

function layer_size_Callback(hObject, eventdata, handles)

$\%$ hObject handle to layer_size (see GCBO)

$\%$ eventdata reserved - to be defined in a future version of MATLAB

$\%$ handles structure with handles and user data (see GUIDATA)

$\%$ Hints: get(hObject,'String') returns contents of layer_size as text

$\% \quad$ str2double(get(hObject,'String')) returns contents of layer_size as a double

val $=$ get(handles.layer,'Value');

if val $==$ handles.model.structure.num_layers

handles.model.structure.layersize.layer(val) $=1$;

else

handles.model.structure.layersize.layer(val) = str2double $($ get $($ hObject,'String'));

end

set(handles.layer_size,'String',num2str(handles.model.structure.layersize.layer(val)));

set(handles.save_model,'Enable','off'); \% disable save Model button until Network created

guidata(hObject, handles);

function bias_Callback(hObject, eventdata, handles)

$\%$ hObject handle to bias (see GCBO)

$\%$ eventdata reserved - to be defined in a future version of MATLAB

$\%$ handles structure with handles and user data (see GUIDATA)

$\%$ Hints: get(hObject,'String') returns contents of bias as text

$\% \quad$ str2double(get(hObject,'String')) returns contents of bias as a double

val = get(handles.layer,'Value');

handles.model.structure.bias.layer(val) = str2double $($ get $($ hObject,'String' $))$;

set(handles.save_model,'Enable','off'); \% disable save Model button until Network created

guidata(hObject, handles);

$\%$--- Executes on selection change in transfer function.

function transfer_function_Callback(hObject, eventdata, handles)

$\%$ hObject handle to transfer_function (see GCBO)

$\%$ eventdata reserved - to be defined in a future version of MATLAB 


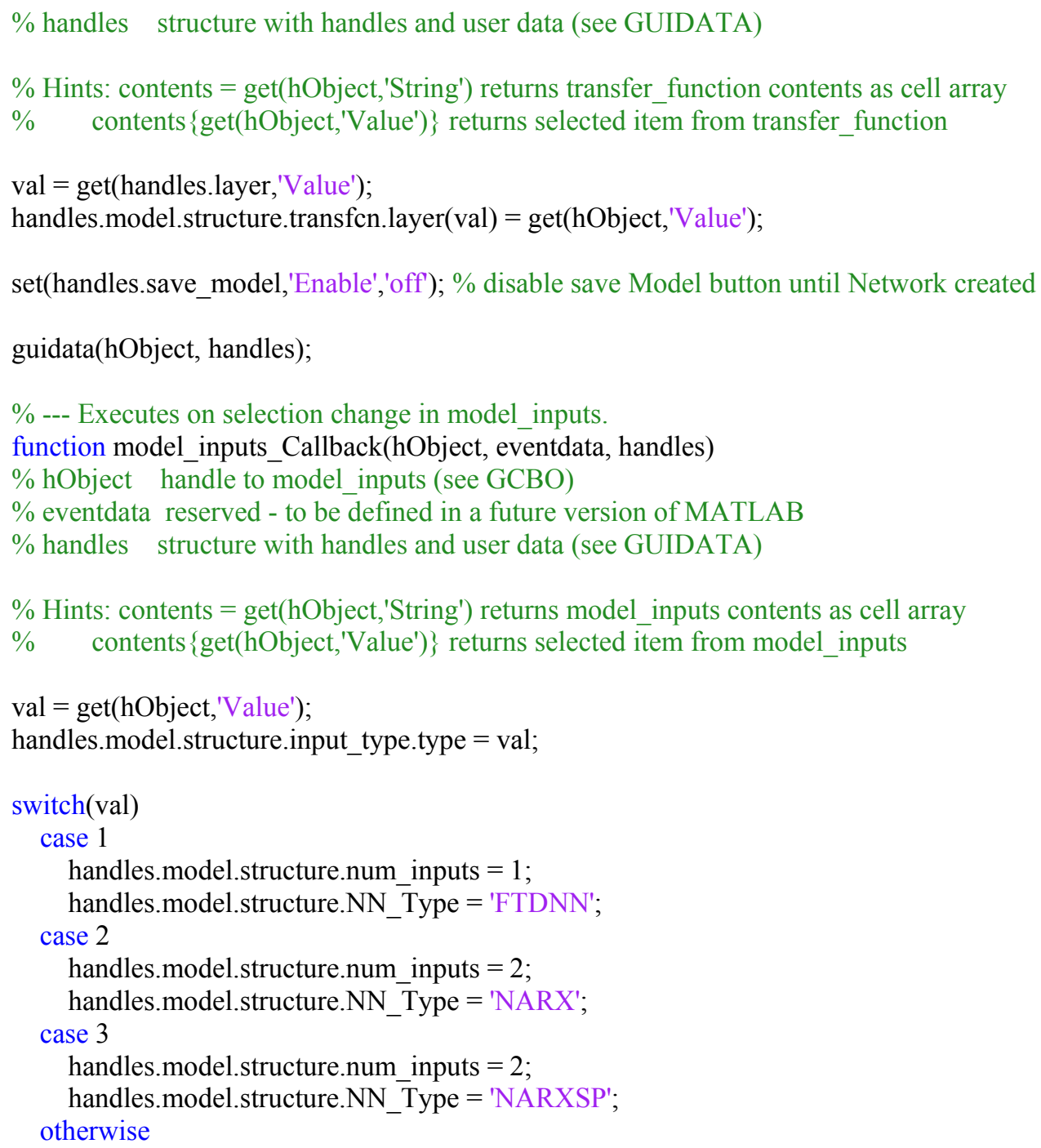


handles.output $=$ hObject;

guidata(hObject, handles);

function model_yp_delays_Callback(hObject, eventdata, handles)

$\%$ hObject handle to model yp delays (see GCBO)

$\%$ eventdata reserved - to be defined in a future version of MATLAB

$\%$ handles structure with handles and user data (see GUIDATA)

$\%$ Hints: get(hObject,'String') returns contents of model_yp_delays as text

$\% \quad$ str2double(get(hObject,'String')) returns contents of model_yp_delays as a double

handles.model.structure.input_type.ypdelays = str2double $($ get $($ hObject,'String'));

handles.output $=$ hObject;

guidata(hObject, handles);

function model_ym_delays_Callback(hObject, eventdata, handles)

$\%$ hObject handle to model_ym_delays (see GCBO)

$\%$ eventdata reserved - to be defined in a future version of MATLAB

$\%$ handles structure with handles and user data (see GUIDATA)

$\%$ Hints: get(hObject,'String') returns contents of model ym delays as text

$\% \quad$ str2double(get(hObject,'String')) returns contents of model_ym_delays as a double

handles.model.structure.input_type.ymdelays $=$ str2double $($ get $($ hObject,'String' $))$;

set(handles.save_model,'Enable','off'); \% disable save Model button until Network created

handles.output $=$ hObject;

guidata(hObject, handles);

$\%$--- Executes on button press in scale u.

function scale_u_Callback(hObject, eventdata, handles)

$\%$ hObject handle to scale_u (see GCBO)

$\%$ eventdata reserved - to be defined in a future version of MATLAB

$\%$ handles structure with handles and user data (see GUIDATA)

$\%$ Hint: get(hObject,'Value') returns toggle state of scale_u

handles.model.structure.input_type.scaling.scale_u = get(hObject,'Value');

handles.output $=$ hObject;

guidata(hObject, handles);

$\%$--- Executes on button press in scale yp.

function scale_yp_Callback(hObject, eventdata, handles) 
$\%$ hObject handle to scale_yp (see GCBO)

$\%$ eventdata reserved - to be defined in a future version of MATLAB

$\%$ handles structure with handles and user data (see GUIDATA)

\% Hint: get(hObject,'Value') returns toggle state of scale_yp

handles.model.structure.input_type.scaling.scale_yp = get(hObject,'Value');

handles.output $=$ hObject;

guidata(hObject, handles);

function max_yp_Callback(hObject, eventdata, handles)

$\%$ hObject handle to max_yp (see GCBO)

$\%$ eventdata reserved - to be defined in a future version of MATLAB

$\%$ handles structure with handles and user data (see GUIDATA)

\% Hints: get(hObject,'String') returns contents of max_yp as text

$\% \quad$ str2double(get(hObject,'String')) returns contents of max_yp as a double

handles.model.structure.input_type.scaling.yp_max = str2double(get(hObject,'String'));

handles.output $=$ hObject;

guidata(hObject, handles);

function max_ym_Callback(hObject, eventdata, handles)

$\%$ hObject handle to max_ym (see GCBO)

$\%$ eventdata reserved - to be defined in a future version of MATLAB

$\%$ handles structure with handles and user data (see GUIDATA)

\% Hints: get(hObject,'String') returns contents of max_ym as text

$\% \quad$ str2double(get(hObject,'String')) returns contents of max_ym as a double

handles.model.structure.input_type.scaling.ym_max $=$ str2double(get(hObject,'String'));

handles.output $=$ hObject;

guidata(hObject, handles);

$\%$--- Executes on selection change in model_weight_init_method.

function model_weight_init_method_Callback(hObject, eventdata, handles)

$\%$ hObject handle to model_weight_init_method (see GCBO)

$\%$ eventdata reserved - to be defined in a future version of MATLAB

$\%$ handles structure with handles and user data (see GUIDATA)

$\%$ Hints: contents $=$ get $($ hObject,'String') returns model_weight_init_method contents as cell array

$\% \quad$ contents $\{$ get(hObject,'Value')\} returns selected item from model_weight_init_method

handles.model.structure.weight_init.method = get(hObject,'Value'); 
handles.output $=$ hObject;

guidata(hObject, handles);

$\%$--- Executes on button press in model_init_weights.

function model_init_weights_Callback(hobject, eventdata, handles)

$\%$ hObject handle to model_init_weights (see GCBO)

$\%$ eventdata reserved - to be defined in a future version of MATLAB

$\%$ handles structure with handles and user data (see GUIDATA)

\% Hint: get(hObject,'Value') returns toggle state of model_init_weights

handles.model.structure.weight_init.initialize = get(hObject,'Value');

handles.output $=$ hObject;

guidata(hObject, handles);

$\%$--- Executes on selection change in model rand weight type.

function model_rand_weight_type_Callback(hObject, eventdata, handles)

$\%$ hObject handle to model_rand_weight_type (see GCBO)

$\%$ eventdata reserved - to be defined in a future version of MATLAB

$\%$ handles structure with handles and user data (see GUIDATA)

$\%$ Hints: contents $=$ get $($ hObject,'String') returns model_rand_weight type contents as cell array

$\% \quad$ contents $\{$ get(hObject,'Value') $\}$ returns selected item from model_rand_weight_type

handles.model.structure.weight_init.random.range = get(hObject,'Value');

handles.output $=$ hObject;

guidata(hObject, handles);

function model_nw_u_lower_Callback(hObject, eventdata, handles)

$\%$ hObject handle to model_nw_u_lower (see GCBO)

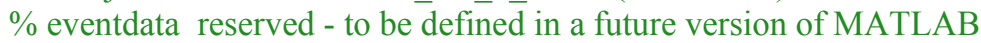

$\%$ handles structure with handles and user data (see GUIDATA)

\% Hints: get(hObject,'String') returns contents of model_nw_u_lower as text

$\% \quad$ str2double(get(hObject,'String')) returns contents of model_nw_u_lower as a double

handles.model.structure.weight_init.nw.u_lower = str2double(get(hObject,'String'));

handles.output $=$ hObject;

guidata(hObject, handles);

function model_nw_yp_lower_Callback(hObject, eventdata, handles)

$\%$ hObject handle to model_nw_yp_lower (see GCBO) 


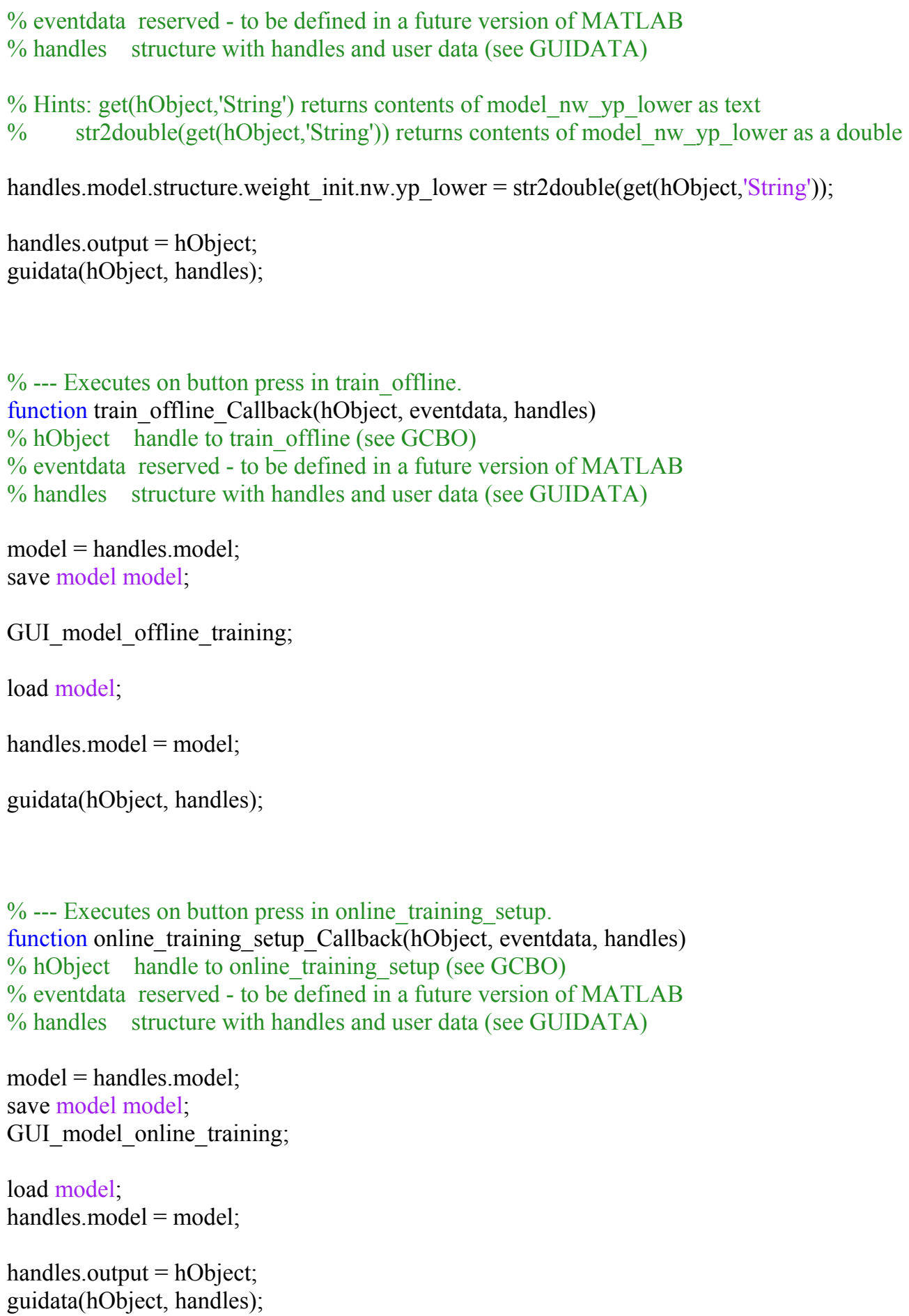

$\%$--- Executes when user attempts to close figure1.

function figure1_CloseRequestFcn(hObject, eventdata, handles)

$\%$ hObject handle to figure1 (see GCBO)

$\%$ eventdata reserved - to be defined in a future version of MATLAB

$\%$ handles structure with handles and user data (see GUIDATA) 


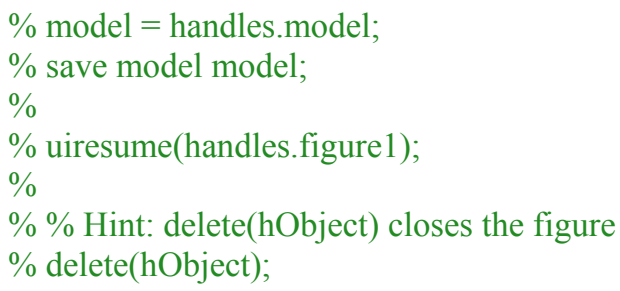

$\%$--- Executes on button press in ok_button.

function ok_button_Callback(hObject, eventdata, handles)

$\%$ hObject handle to ok button (see GCBO)

$\%$ eventdata reserved - to be defined in a future version of MATLAB

$\%$ handles structure with handles and user data (see GUIDATA)

model $=$ handles.model;

\%setup model;

\%initialize model weights; \% initalizes weights

save model model;

handles.model $=$ model;

uiresume(handles.figure1);

$\%$--- Executes on button press in Initalize_Weights.

function Initalize_Weights_Callback(hObject, eventdata, handles)

$\%$ hObject handle to Initalize_Weights (see GCBO)

$\%$ eventdata reserved - to be defined in a future version of MATLAB

$\%$ handles structure with handles and user data (see GUIDATA)

model $=$ handles.model;

save model model;

setup_model;

load model;

handles.model $=$ model;

set(handles.save_model,'Enable','on') \% Enable Save model button

handles.output $=$ hObject;

guidata(hObject, handles);

$\%$--- Executes on button press in Import_Training_Set.

function Import_Training_Set_Callback(hObject, eventdata, handles)

$\%$ hObject handle to Import Training Set (see GCBO)

$\%$ eventdata reserved - to be defined in a future version of MATLAB

$\%$ handles structure with handles and user data (see GUIDATA)

[filename, pathname, filterindex] = uigetfile('*.xlsx', 'Choose Excel 2007 file containing training set'); 


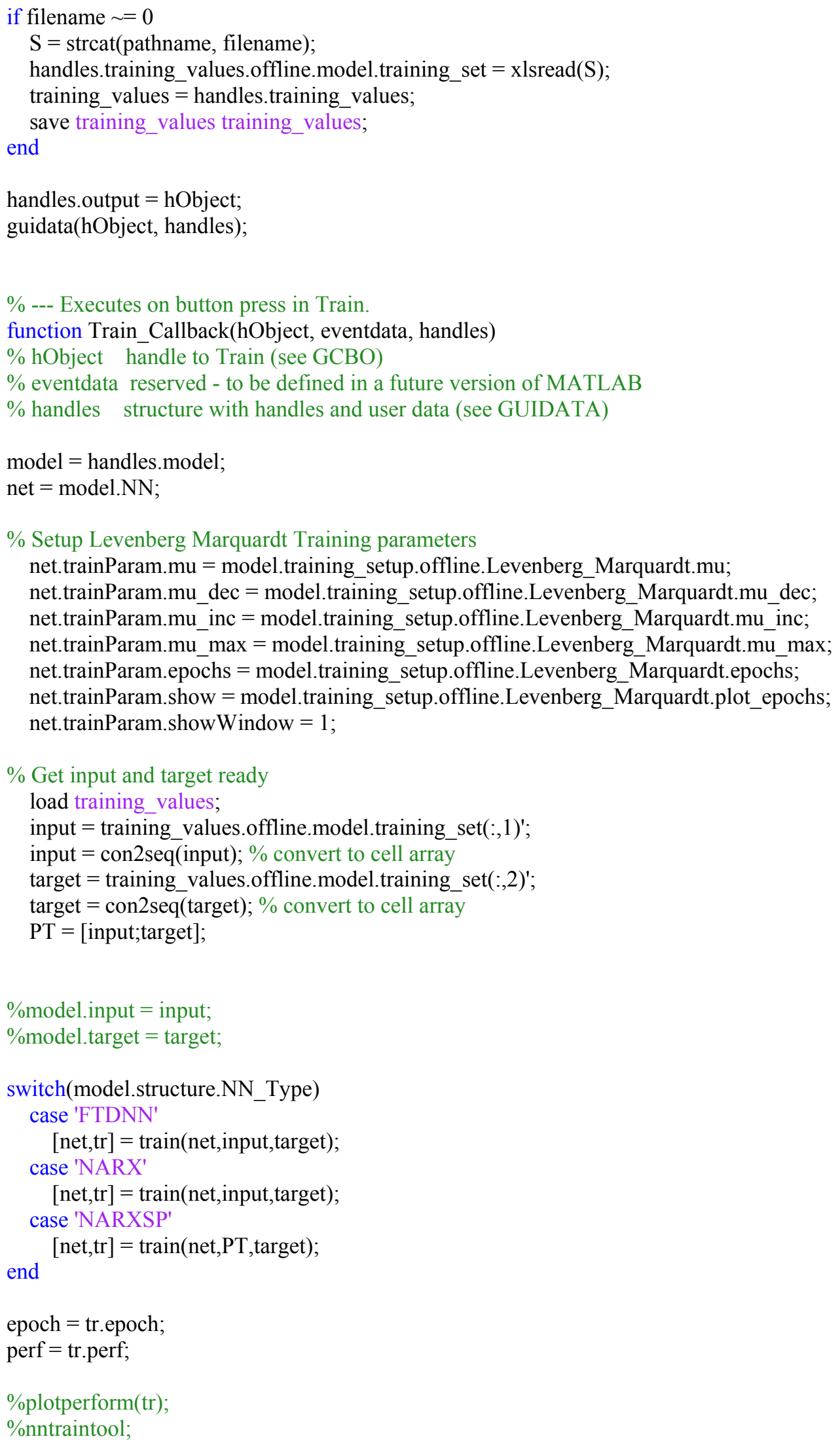




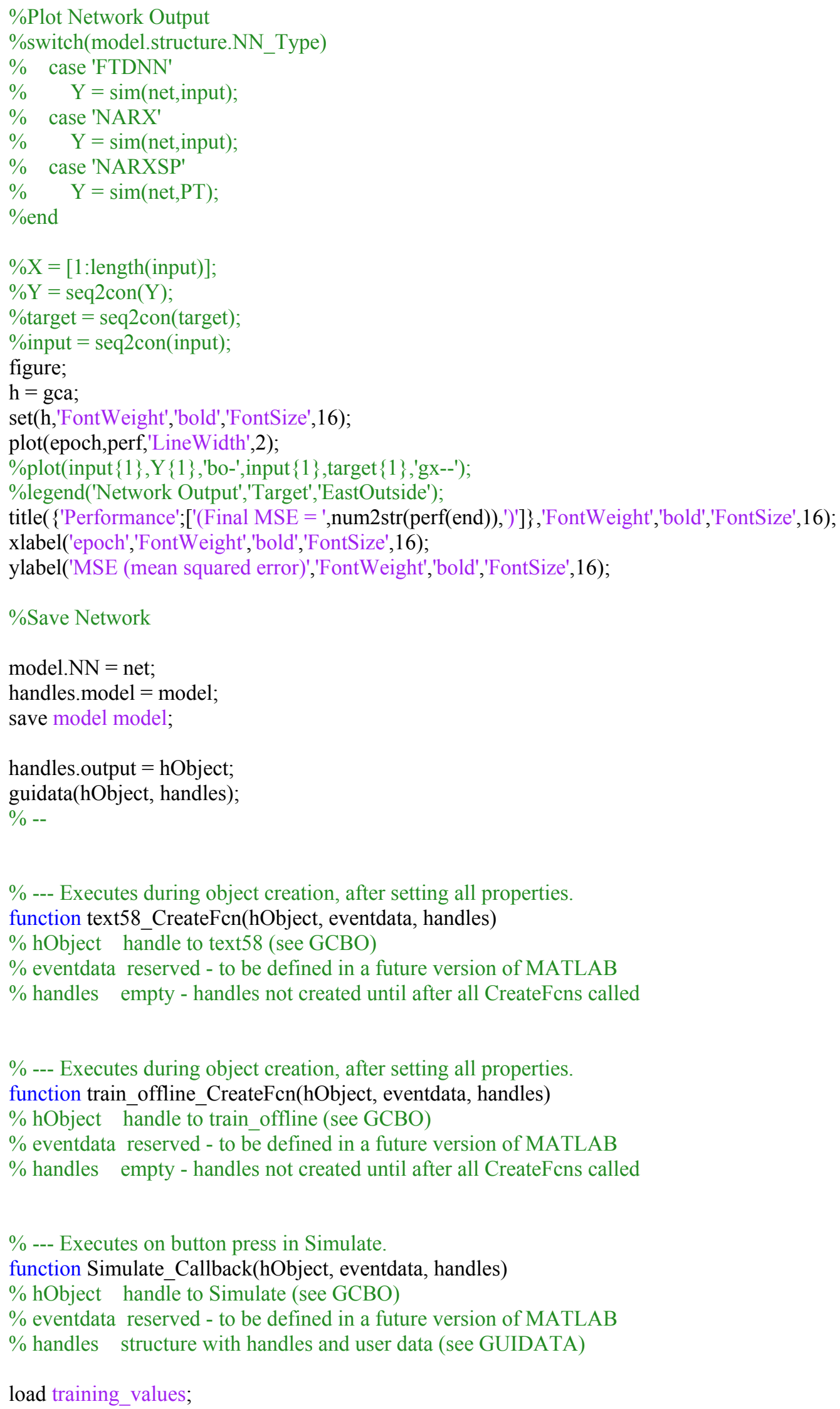




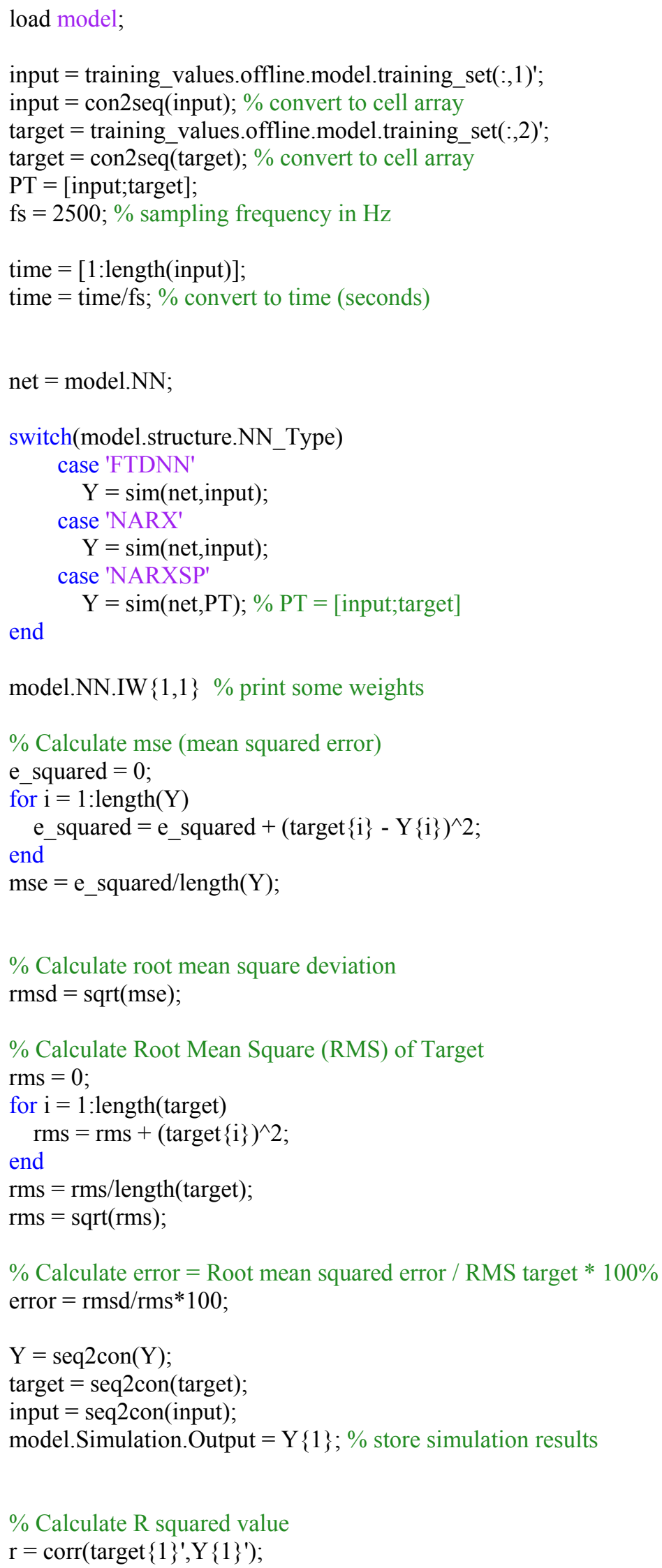




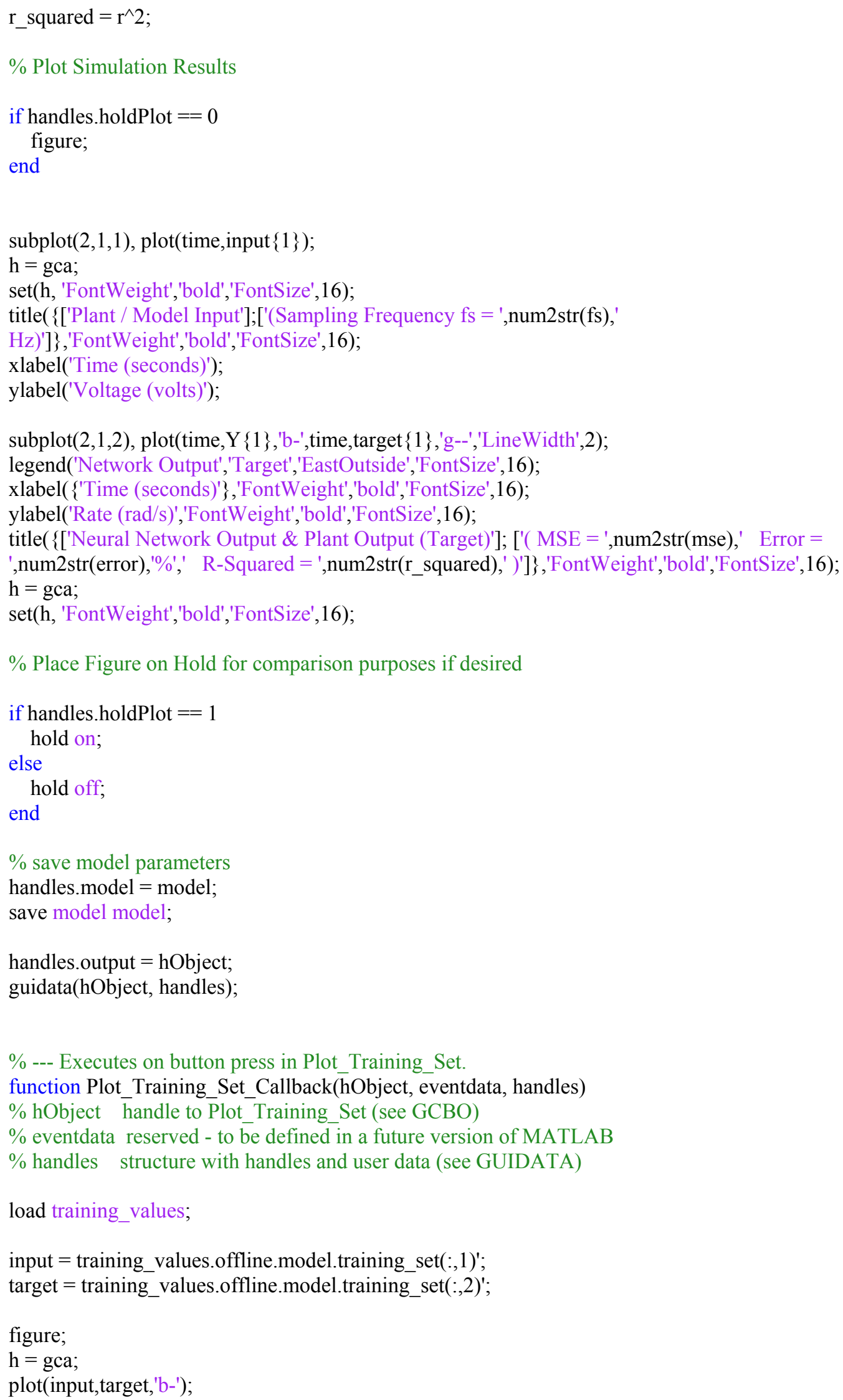


xlabel('Model Input','FontWeight','bold','FontSize',16);

ylabel('Model Target','FontWeight','bold','FontSize',16);

title('Plant Response','FontWeight','bold','FontSize',16);

set(h, 'FontWeight','bold','FontSize',16);

$\%$--- Executes on button press in InputTargetPlotButton.

function InputTargetPlotButton_Callback(hObject, eventdata, handles)

$\%$ hObject handle to InputTargetPlotButton (see GCBO)

$\%$ eventdata reserved - to be defined in a future version of MATLAB

$\%$ handles structure with handles and user data (see GUIDATA)

load training_values;

$\mathrm{fs}=2500 ; \%$ Sampling Frequency $(\mathrm{Hz})$

input $=$ training_values.offline.model.training_set(:,1)';

target $=$ training_values.offline.model.training_set $(:, 2)^{\prime}$;

$\mathrm{X}=[1$ :length(input) $]$;

time $=\mathrm{X} / \mathrm{fs}$;

figure;

$\mathrm{h}=\mathrm{gca}$;

plot(time, input,'b-',time,target,'g--','LineWidth',2);

legend('Plant Input','Plant Output,'EastOutside','FontSize',16);

xlabel('Time (seconds)','FontWeight','bold','FontSize',16);

ylabel('Input: Voltage (volts) Output: Rate (rad/sec)','FontWeight','bold','FontSize',16);

title('Plant Response','FontWeight','bold','FontSize',16);

set(h, 'FontWeight','bold','FontSize',16);

$\%$--- Executes on button press in pushButtonFrequencyResponse.

function pushButtonFrequencyResponse_Callback(hObject, eventdata, handles)

$\%$ hObject handle to pushButtonFrequencyResponse (see GCBO)

$\%$ eventdata reserved - to be defined in a future version of MATLAB

$\%$ handles structure with handles and user data (see GUIDATA)

load training_values;

input $=$ training_values.offline.model.training_set(:,1)';

target $=$ training_values.offline.model.training_set $(:, 2)^{\prime}$;

$\mathrm{X}=[1$ :length(input) $]$;

$\mathrm{fs}=2500 ; \%$ sampling frequency

$\%$ Compute the Frequency Domain Signal for the input

$\mathrm{NFFT}=2^{\wedge}$ nextpow2(fs); $\%$ Next power of 2 from length of $y$

$\mathrm{Y}=\mathrm{fft}($ input,NFFT)/fs;

$\mathrm{f}=\mathrm{fs} / 2 *$ linspace $(0,1, \mathrm{NFFT} / 2+1)$;

$\%$ Plot the single-sided Frequency amplitude spectrum.

figure; 


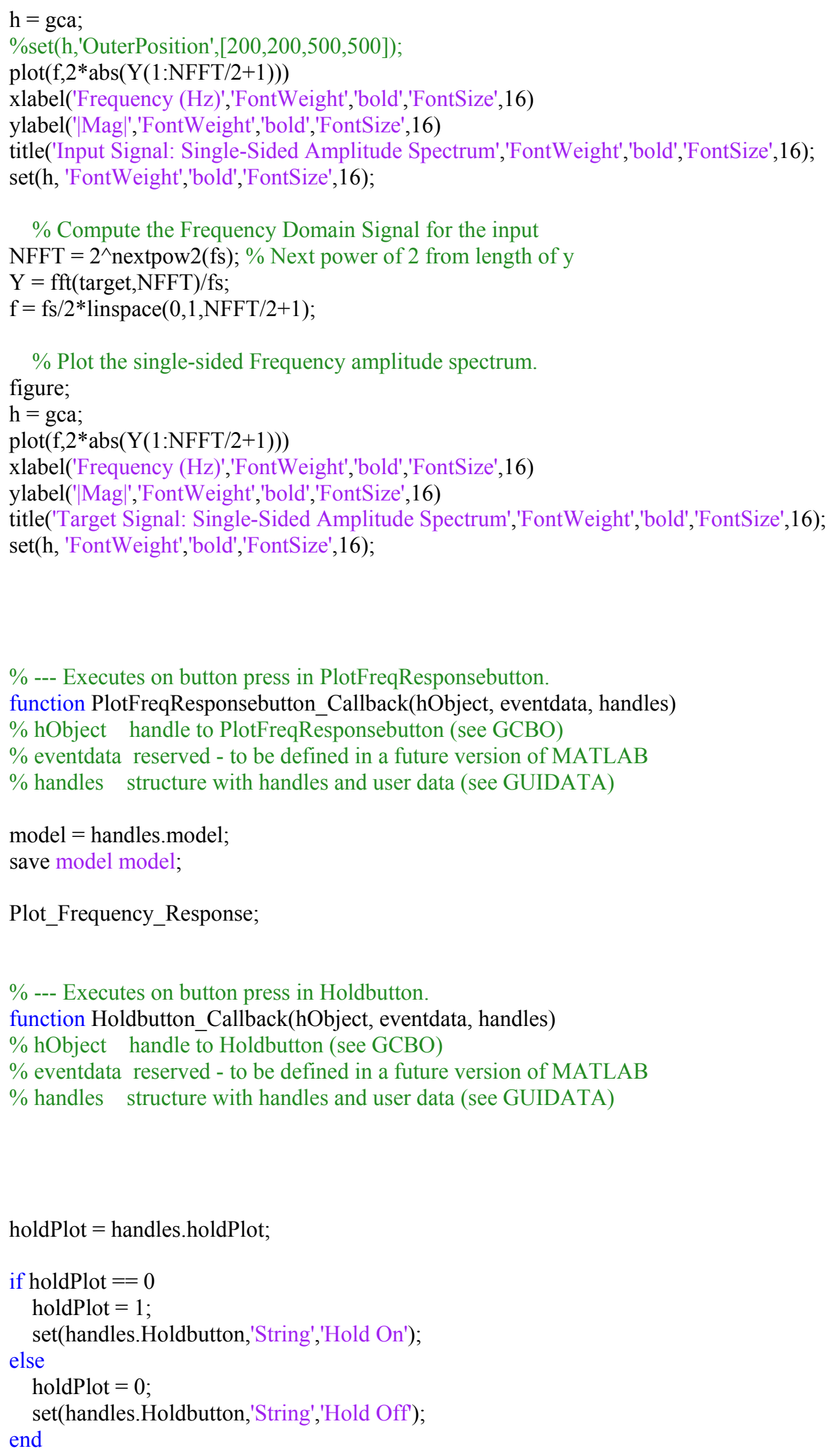


handles.holdPlot $=$ holdPlot;

handles.output $=$ hObject;

guidata(hObject, handles); 


\section{Initialize GUI Model:}

\section{File: initialize_GUI_model.m}

\section{Description: Initialize model settings and structure.}

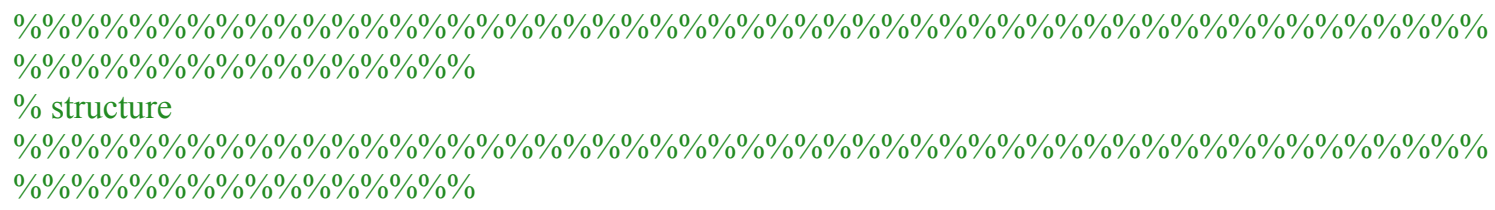

model.structure.weight init.method $=1$;

model.structure.weight_init.initialize $=1$;

model.structure.weight_init.random.range $=2$;

model.structure.weight_init.random.range_value $=1$;

model.structure.weight_init.nw.u lower $=0$;

model.structure.weight_init.nw.u_upper $=0$;

model.structure.weight_init.nw.yp_lower $=0$; model.structure.weight_init.nw.yp_upper $=0$; model.structure.weight_init.nw.ym_lower $=0$; model.structure.weight_init.nw.ym_upper $=0$;

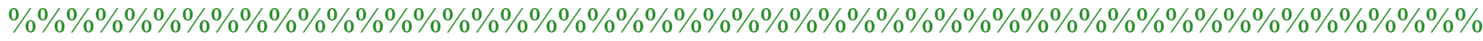
$\% \% \% \% \% \% \% \% \% \% \% \% \% \% \% \%$

$\%$ initialize GUI model offline training variables 
\%\%\%\%\%\%\%\%\%\%\%\%\%\%\%\%\%\%\%\%\%\%\%\%\%\%\%\%\%\%\%\%\%\%\%\%\%\%\%\%\%\%\%\%\%\%\%\%\%\%\%

$\% \% \% \% \% \% \% \% \% \% \% \% \% \% \% \%$

$\%$ Levenberg Marquardt

model.training_setup.offline.Levenberg_Marquardt.mu $=.001$;

model.training_setup.offline.Levenberg_Marquardt.mu_dec $=.1$;

model.training_setup.offline.Levenberg_Marquardt.mu_inc $=10$;

model.training_setup.offline.Levenberg_Marquardt.mu_max $=1 \mathrm{e} 10$;

model.training_setup.offline.Levenberg_Marquardt.epochs $=100 ; \%$ epochs

model.training_setup.offline.Levenberg_Marquardt.plot_epochs $=5 ; \%$ plot every one epoch

$\%$ offline input

$\%$ General settings

model.training_setup.offline.input_setup.type $=1$;

model.training_setup.offline.input_setup.samp_freq $=1$;

model.training_setup.offline.input_setup.SMSF.num_samples $=0$;

model.training_setup.offline.input_setup.signal $=0$;

$\%$ SMSF settings

model.training_setup.offline.input_setup.SMSF.amplitude $=0$;

model.training_setup.offline.input_setup.SMSF.num_freq $=1$;

model.training_setup.offline.input_setup.SMSF.freq $\overline{(1)}=0$;

model.training_setup.offline.input_setup.SMSF.freq $(2)=0$;

model.training_setup.offline.input_setup.SMSF.freq $(3)=0$;

model.training_setup.offline.input_setup.SMSF.freq $(4)=0$;

model.training_setup.offline.input_setup.SMSF.freq(5) $=0$;

model.training_setup.offline.input_setup.SMSF.freq $(6)=0$;

\% SMSF_w_MFE settings

model.training_setup.offline.input_setup.SMSF_w_MFE.single_freq.num_freq $=1$;

model.training_setup.offline.input_setup.SMSF_W_MFE.single_freq.num_samples $=0$;

model.training_setup.offline.input_setup.SMSF_w_MFE.single_freq.amplitude $=0$;

model.training_setup.offline.input_setup.SMSF_w_MFE.single_freq.freq $(1)=0$;

model.training_setup.offline.input_setup.SMSF_W_MFE.single_freq.freq $(2)=0$;

model.training_setup.offline.input_setup.SMSF_w_MFE.single_freq.freq(3) $=0$;

model.training_setup.offline.input_setup.SMSF_w_MFE.single_freq.freq $(4)=0$;

model.training_setup.offline.input_setup.SMSF_W_MFE.single_freq.freq(5) $=0$;

model.training_setup.offline.input_setup.SMSF_w_MFE.single_freq.freq $(6)=0$;

model.training_setup.offline.input_setup.SMSF_w_MFE.mult_freq.num_freq $=1$;

model.training_setup.offline.input_setup.SMSF_w_MFE.mult_freq.num_samples $=0$;

model.training_setup.offline.input_setup.SMSF_W_MFE.mult_freq.amplitude $=0$;

model.training_setup.offline.input_setup.SMSF_W_MFE.mult_freq.freq $(1)=0$;

model.training_setup.offline.input_setup.SMSF_w_MFE.mult_freq.freq $(2)=0$;

model.training_setup.offline.input_setup.SMSF_w_MFE.mult_freq.freq $(3)=0$;

model.training_setup.offline.input_setup.SMSF_w_MFE.mult_freq.freq $(4)=0$;

model.training_setup.offline.input_setup.SMSF_w_MFE.mult_freq.freq $(5)=0$;

model.training_setup.offline.input_setup.SMSF_w_MFE.mult_freq.freq $(6)=0$;

$\%$ MFW settings

model.training_setup.offline.input_setup.MFW.num_samples $=0$;

model.training_setup.offline.input_setup.MFW.amplitude $=0$;

model.training_setup.offline.input_setup.MFW.num_freq $=1$;

model.training_setup.offline.input_setup.MFW.freq $\overline{(1})=0$; 
model.training_setup.offline.input_setup.MFW.freq $(2)=0$; model.training_setup.offline.input_setup.MFW.freq $(3)=0$; model.training_setup.offline.input_setup.MFW.freq $(4)=0$; model.training_setup.offline.input_setup.MFW.freq $(5)=0$; model.training_setup.offline.input_setup.MFW.freq $(6)=0$;

$\%$ chirp settings

model.training_setup.offline.input_setup.chirp.amplitude $=0$; model.training_setup.offline.input_setup.chirp.time $=0$;

$\%$ NSCRS settings

model.training_setup.offline.input_setup.NSCRS.num_samples $=0$; model.training_setup.offline.input_setup.NSCRS.min_amp $=0$; model.training_setup.offline.input_setup.NSCRS.max_amp $=0$; model.training_setup.offline.input_setup.NSCRS.interval $=0$;

$\%$ RSLC settings

model.training_setup.offline.input_setup.RSLC.num_samples $=0$;

model.training_setup.offline.input_setup.RSLC.min_amp $=0$;

model.training_setup.offline.input_setup.RSLC.max_amp $=0$;

model.training_setup.offline.input_setup.RSLC.min_interval $=0$;

model.training_setup.offline.input_setup.RSLC.max_interval $=0$;

\%RSLC_W_DAS settings

model.training_setup.offline.input_setup.RSLC_w_DAS.num_segments $=1$; model.training_setup.offline.input_setup.RSLC_w_DAS.min_interval $=0$; model.training_setup.offline.input_setup.RSLC_w_DAS.max_interval $=0$; model.training_setup.offline.input_setup.RSLC_W_DAS.seg $(\overline{1}) \cdot \min$ amp $=0$; model.training_setup.offline.input_setup.RSLC_w_DAS.seg(2).min_amp $=0$; model.training_setup.offline.input_setup.RSLC_w_DAS.seg(3).min_amp $=0$; model.training_setup.offline.input_setup.RSLC_w_DAS.seg(4).min_amp $=0$; model.training_setup.offline.input_setup.RSLC_w_DAS.seg(1).max_amp $=0$; model.training_setup.offline.input_setup.RSLC_w_DAS.seg(2).max_amp $=0$; model.training_setup.offline.input_setup.RSLC_w_DAS.seg(3).max_amp $=0$; model.training_setup.offline.input_setup.RSLC_w_DAS.seg(4).max_amp $=0$; model.training_setup.offline.input_setup.RSLC_w_DAS.seg(1).num_samples $=0$; model.training_setup.offline.input_setup.RSLC_w_DAS.seg(2).num_samples $=0$; model.training_setup.offline.input_setup.RSLC_w_DAS.seg(3).num_samples $=0$; model.training_setup.offline.input_setup.RSLC_w_DAS.seg(4).num_samples $=0$;

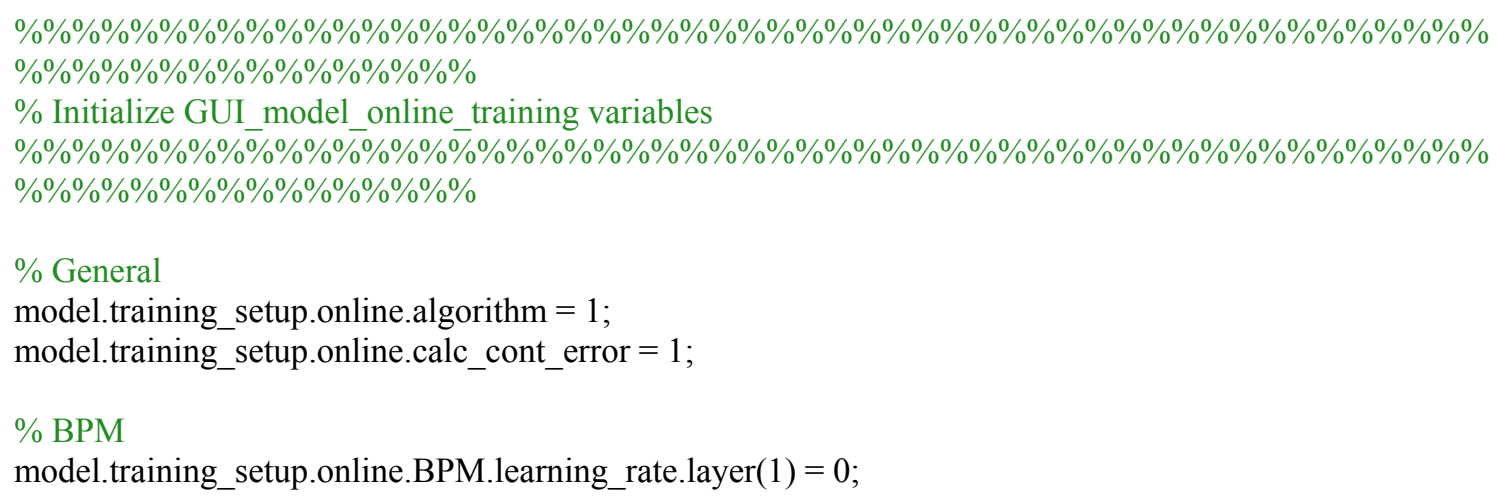


model.training_setup.online.BPM.learning_rate.layer $(2)=0$;

model.training_setup.online.BPM.momentum_factor.layer $(1)=0$;

model.training_setup.online.BPM.momentum_factor.layer $(2)=0$;

$\% \mathrm{RP}$

model.training_setup.online.RP.init_wt_change $=0$;

model.training_setup.online.RP.max_wt_change $=0$;

model.training_setup.online.RP.inc_wt_change $=0$;

model.training_setup.online.RP.dec_wt_change $=0$;

$\%$ LRLS

model.training_setup.online.LRLS.forget_factor $=0$;

model.training_setup.online.LRLS.A.layer(1) $=0$;

model.training_setup.online.LRLS.A.layer $(2)=0$;

$\%$ IGLS

model.training_setup.online.IGLS.forget_factor $=0$;

model.training_setup.online.IGLS.A.layer $(1)=0$;

model.training_setup.online.IGLS.learning_rate.layer(2) $=0$;

model.training_setup.online.IGLS.momentum_factor.layer $(2)=0$;

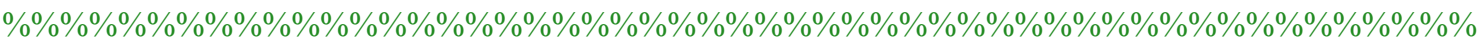
$\% \% \% \% \% \% \% \% \% \% \% \% \% \% \%$

$\%$ Initialize GUI model Simulation Results

\%\%\%\%\%\%\%\%\%\%\%\%\%\%\%\%\%\%\%\%\%\%\%\%\%\%\%\%\%\%\%\%\%\%\%\%\%\%\%\%\%\%\%\%\%\%\%\%\%\%\%

$\% \% \% \% \% \% \% \% \% \% \% \% \% \% \% \%$

model.Simulation. Output $=0$;

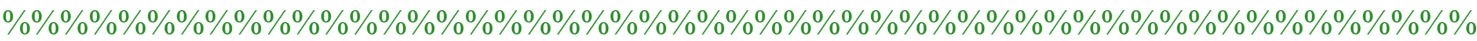
$\% \% \% \% \% \% \% \% \% \% \% \% \% \% \%$

$\%$ Initialize GUI model Plotting Variables

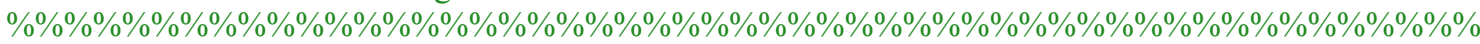

$\% \% \% \% \% \% \% \% \% \% \% \% \% \% \% \%$

model.Plotting.Freq.DataType $=1$;

model.Plotting.Freq.StartFreq $=1$;

model.Plotting.Freq.EndFreq = 1250; 


\title{
Update GUI model:
}

\section{File: update_GUI_model.m}

\section{Description: Update GUI model variables}

\author{
$\%$ structure \\ val $=\{\}$;




\section{Setup model:}

\section{File: setup_model.m}

\section{Description: Creates and Initalizes neural network model}

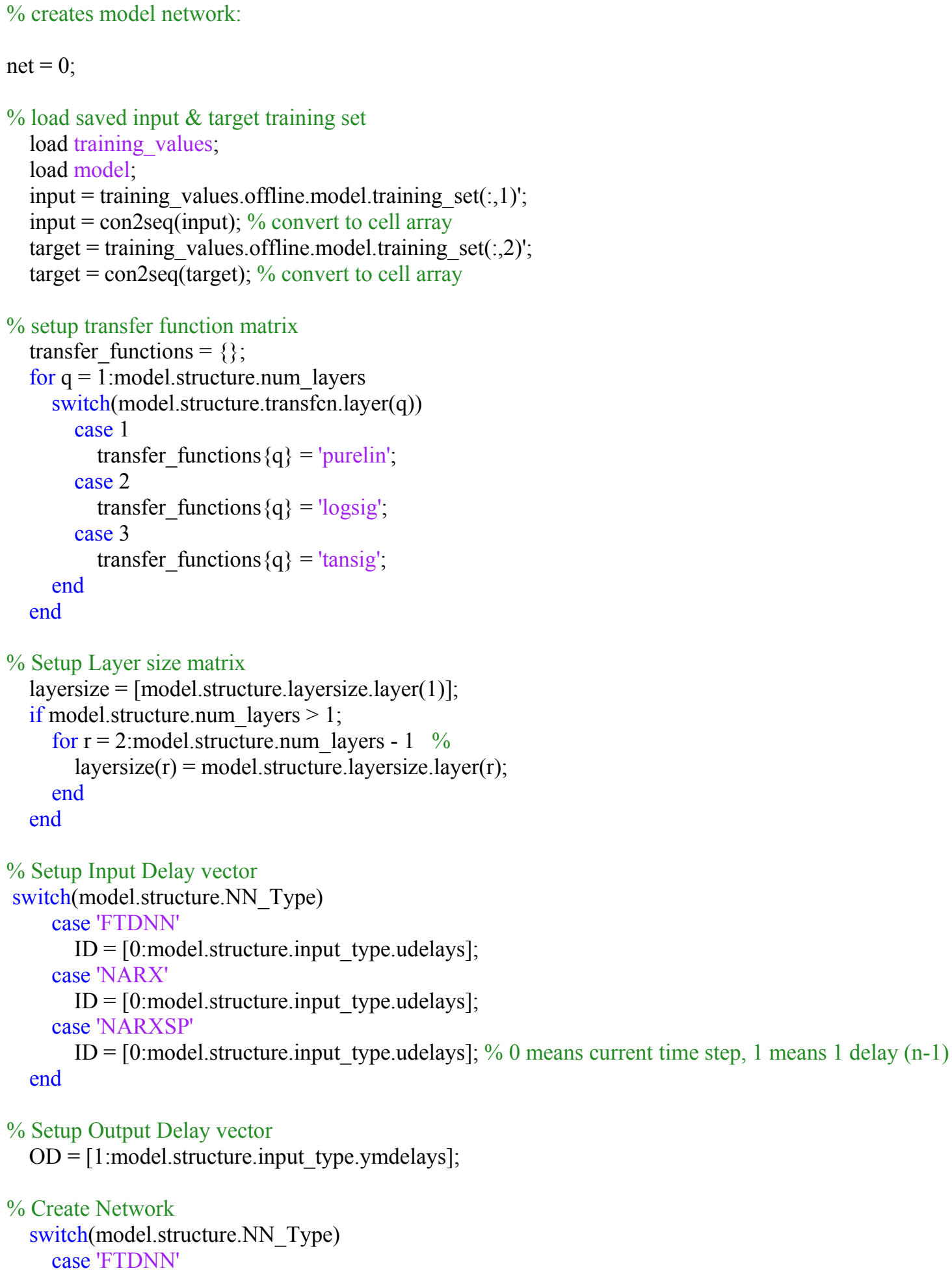




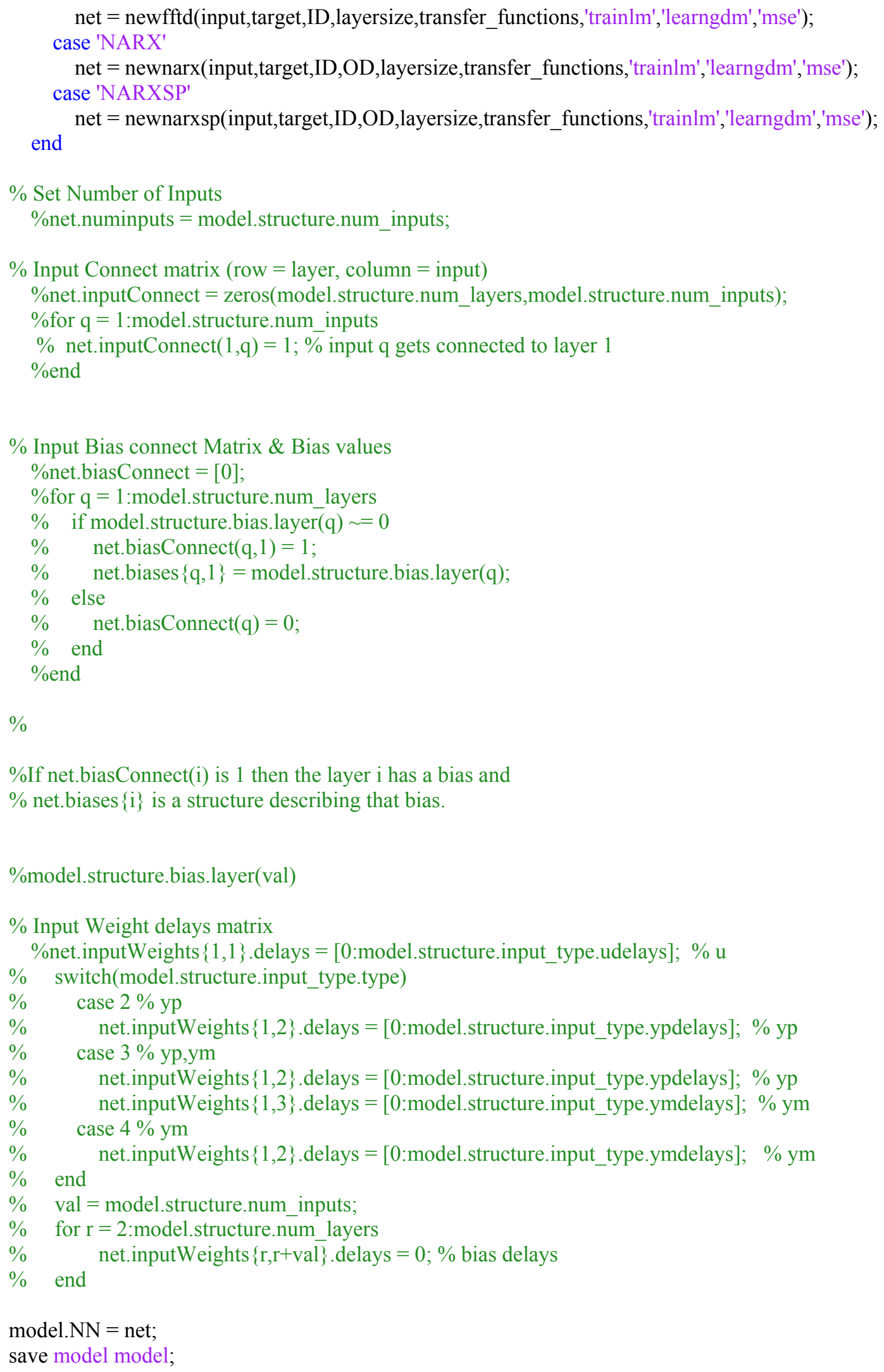




\section{Training Parameter Setup GUI:}

\section{File: GUI_model_offline_training.m}

\section{Description: Graphical User Interface used to set neural network training parameters}

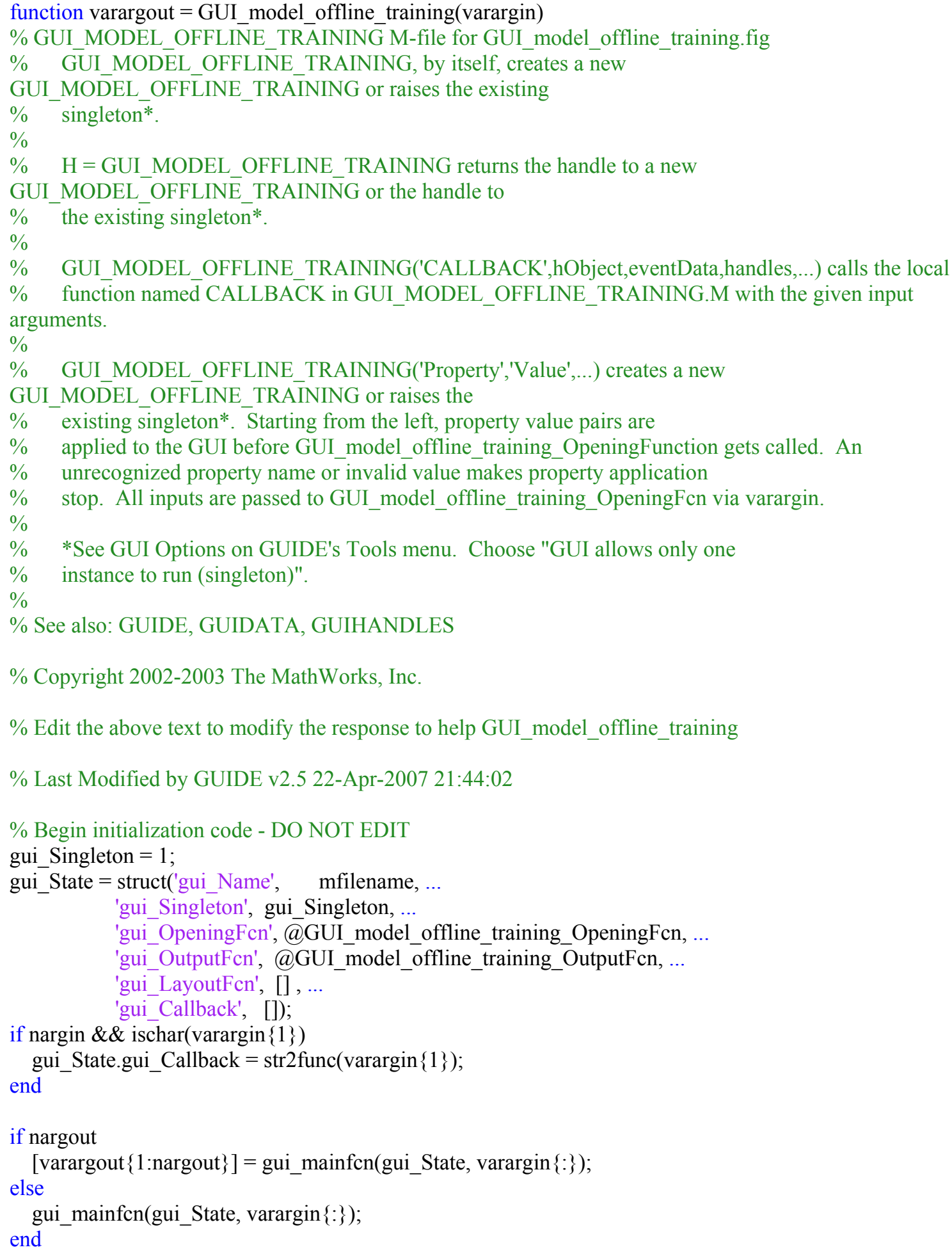


$\%$ End initialization code - DO NOT EDIT

$\%$--- Executes just before GUI_model_offline_training is made visible.

function GUI_model_offline_training_OpeningFen(hObject, eventdata, handles, varargin)

$\%$ This function has no output args, see OutputFen.

$\%$ hObject handle to figure

$\%$ eventdata reserved - to be defined in a future version of MATLAB

$\%$ handles structure with handles and user data (see GUIDATA)

$\%$ varargin command line arguments to GUI_model_offline_training (see VARARGIN)

load model;

handles.model = model;

update_GUI_model_offline;

$\%$ Choose default command line output for GUI_model_offline_training

handles.output $=$ hObject;

$\%$ Update handles structure

guidata(hObject, handles);

$\%$ Make the GUI modal

set(handles.figure1,'WindowStyle','modal')

$\%$ Determine the position of the dialog - centered on the callback figure

$\%$ if available, else, centered on the screen

FigPos=get(0,'DefaultFigurePosition');

OldUnits = get(hObject, 'Units');

set(hObject, 'Units', 'pixels');

OldPos = get(hObject,'Position');

FigWidth = OldPos(3);

FigHeight $=$ OldPos(4);

if isempty(gcbf)

ScreenUnits $=$ get $\left(0,{ }^{\prime} U n i t s '\right)$;

$\operatorname{set}(0$, 'Units','pixels');

ScreenSize $=\operatorname{get}\left(0,{ }^{\prime}\right.$ ScreenSize');

set(0,'Units',ScreenUnits);

FigPos $(1)=1 / 2 *(\operatorname{ScreenSize}(3)-F i g W i d t h)$;

FigPos $(2)=2 / 3 *($ ScreenSize(4)-FigHeight);

else

GCBFOldUnits = get(gcbf,'Units');

set(gcbf,'Units','pixels');

GCBFPos = get(gcbf,'Position');

set(gcbf,'Units',GCBFOldUnits);

FigPos $(1: 2)=[(\operatorname{GCBFPos}(1)+\operatorname{GCBFPos}(3) / 2)-$ FigWidth $/ 2, \ldots$

end

(GCBFPos(2) + GCBFPos(4) / 2) - FigHeight / 2];

FigPos(3:4)=[FigWidth FigHeight];

set(hObject, 'Position', FigPos);

set(hObject, 'Units', OldUnits);

\% UIWAIT makes GUI_model_offline_training wait for user response (see UIRESUME) uiwait(handles.figure1); 


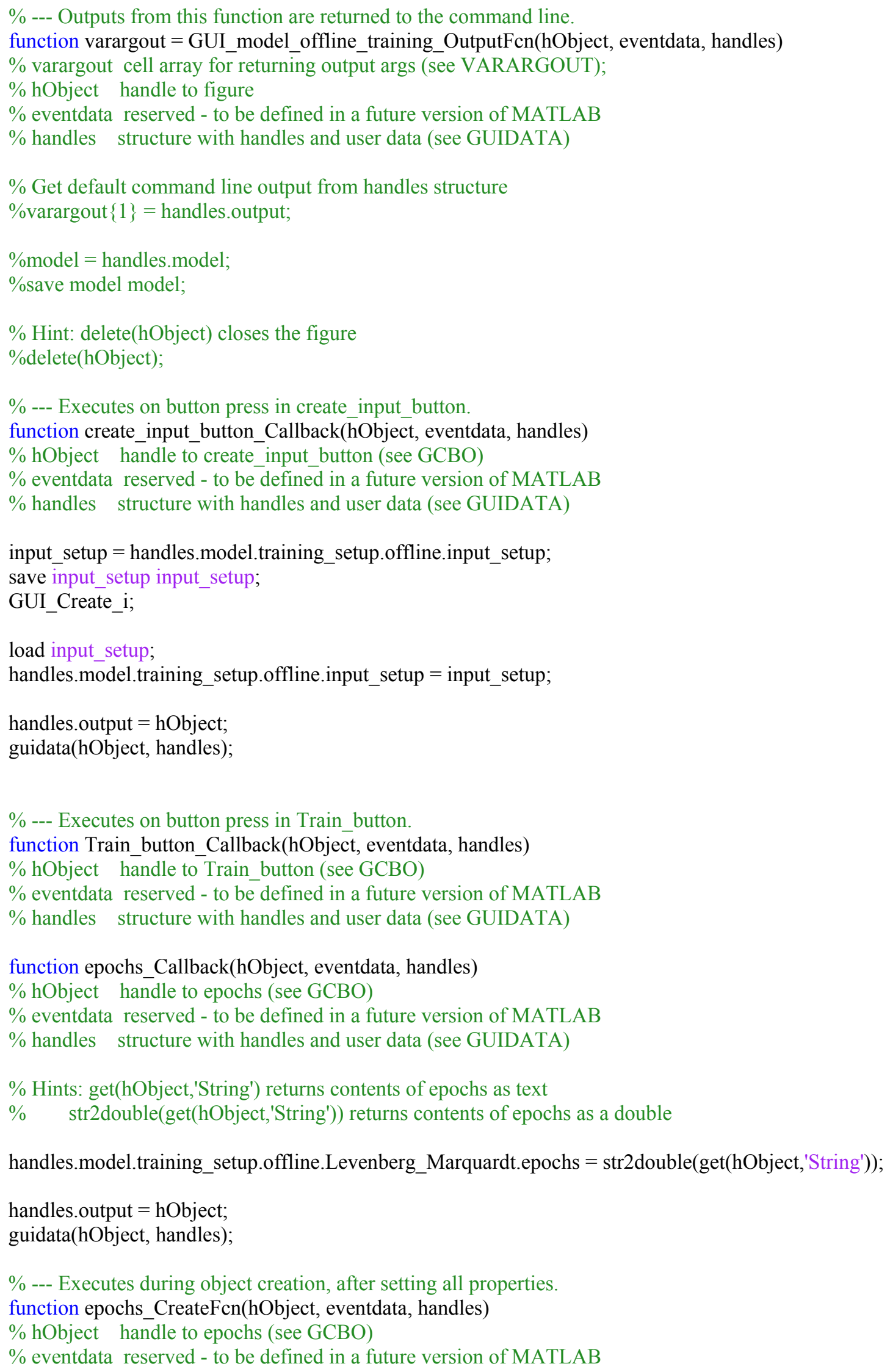




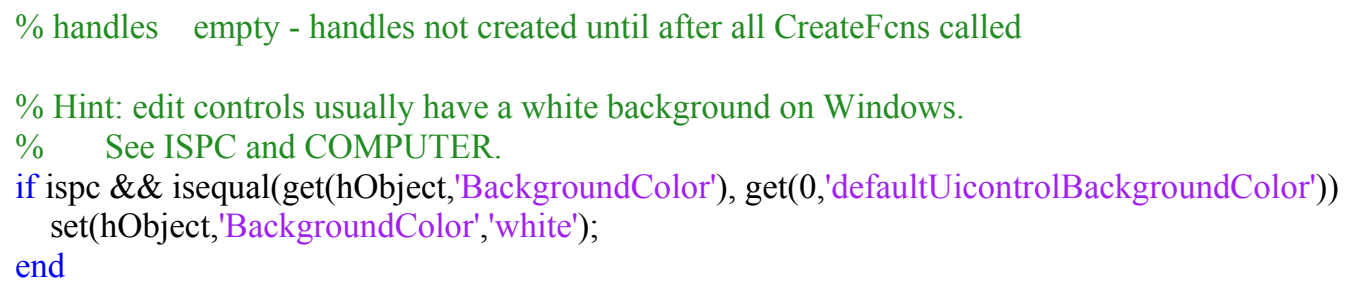


end

$\%$--- Executes on button press in plot_button.

function plot_button_Callback(hObject, eventdata, handles)

$\%$ hObject handle to plot_button (see GCBO)

$\%$ eventdata reserved - to be defined in a future version of MATLAB

$\%$ handles structure with handles and user data (see GUIDATA)

$\%$--- Executes when user attempts to close figure1.

function figure1_CloseRequestFen(hObject, eventdata, handles)

$\%$ hObject handle to figure1 (see GCBO)

$\%$ eventdata reserved - to be defined in a future version of MATLAB

$\%$ handles structure with handles and user data (see GUIDATA)

model $=$ handles.model;

save model model;

uiresume(handles.figure1);

$\%$ Hint: delete(hObject) closes the figure

delete(hObject);

function mu_Callback(hObject, eventdata, handles)

$\%$ hObject handle to mu (see GCBO)

$\%$ eventdata reserved - to be defined in a future version of MATLAB

$\%$ handles structure with handles and user data (see GUIDATA)

$\%$ Hints: get(hObject,'String') returns contents of mu as text

$\% \quad$ str2double(get(hObject,'String')) returns contents of mu as a double

handles.model.training_setup.offline.Levenberg_Marquardt.mu = str2double(get(hObject,'String'));

handles.output $=$ hObject;

guidata(hObject, handles);

$\%$--- Executes during object creation, after setting all properties.

function mu_CreateFcn(hObject, eventdata, handles) 
$\%$ hObject handle to mu (see GCBO)

$\%$ eventdata reserved - to be defined in a future version of MATLAB

$\%$ handles empty - handles not created until after all CreateFcns called

$\%$ Hint: edit controls usually have a white background on Windows.

$\% \quad$ See ISPC and COMPUTER.

if ispc \&\& isequal(get(hObject,'BackgroundColor'), get( 0 ,'defaultUicontrolBackgroundColor')) set(hObject,'BackgroundColor','white'); end

function mu_dec_Callback(hObject, eventdata, handles)

$\%$ hObject handle to mu_dec (see GCBO)

$\%$ eventdata reserved - to be defined in a future version of MATLAB

$\%$ handles structure with handles and user data (see GUIDATA)

$\%$ Hints: get(hObject,'String') returns contents of mu_dec as text

$\% \quad$ str2double(get(hObject,'String')) returns contents of mu_dec as a double

handles.model.training_setup.offline.Levenberg_Marquardt.mu_dec =str2double(get(hObject,'String'));

handles.output $=$ hObject;

guidata(hObject, handles);

$\%$--- Executes during object creation, after setting all properties.

function mu_dec_CreateFcn(hObject, eventdata, handles)

$\%$ hObject hand̄e to mu_dec (see GCBO)

$\%$ eventdata reserved - to be defined in a future version of MATLAB

$\%$ handles empty - handles not created until after all CreateFcns called

$\%$ Hint: edit controls usually have a white background on Windows.

$\% \quad$ See ISPC and COMPUTER.

if ispc \&\& isequal(get(hObject,'BackgroundColor'), get(0,'defaultUicontrolBackgroundColor')) set(hObject,'BackgroundColor','white');

end

function mu_inc_Callback(hObject, eventdata, handles)

$\%$ hObject handle to mu inc (see GCBO)

$\%$ eventdata reserved - to be defined in a future version of MATLAB

$\%$ handles structure with handles and user data (see GUIDATA)

$\%$ Hints: get(hObject,'String') returns contents of mu_inc as text

$\% \quad$ str2double(get(hObject,'String')) returns contents of mu_inc as a double

handles.model.training_setup.offline.Levenberg_Marquardt.mu_inc $=$ str2double(get(hObject,'String'));

handles.output $=$ hObject;

guidata(hObject, handles);

$\%$--- Executes during object creation, after setting all properties.

function mu_inc_CreateFcn(hObject, eventdata, handles)

$\%$ hObject handle to mu inc (see GCBO)

$\%$ eventdata reserved - to be defined in a future version of MATLAB 


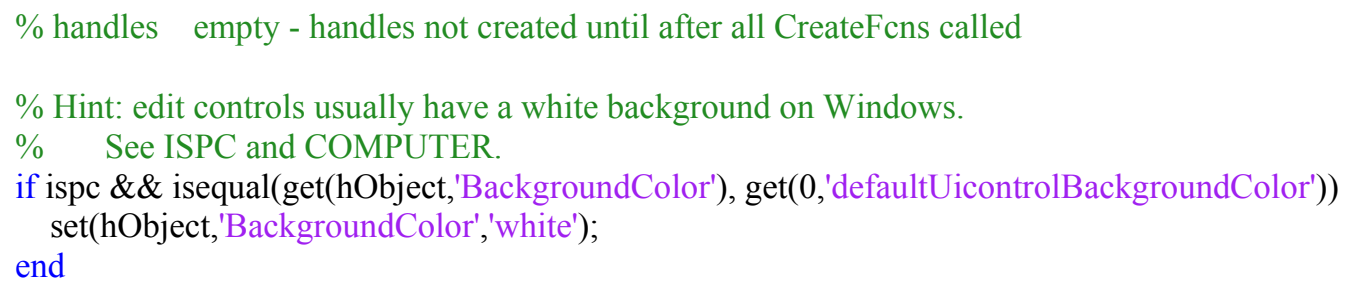


$\% \quad$ str2double(get(hObject,'String')) returns contents of plot_epochs as a double

handles.model.training_setup.offline.Levenberg_Marquardt.plot_epochs = str2double(get(hObject,'String'));

handles.output $=$ hObject;

guidata(hObject, handles);

$\%$--- Executes during object creation, after setting all properties.

function plot_epochs_CreateFcn(hObject, eventdata, handles)

$\%$ hObject handle to plot_epochs (see GCBO)

$\%$ eventdata reserved - to be defined in a future version of MATLAB

$\%$ handles empty - handles not created until after all CreateFcns called

$\%$ Hint: edit controls usually have a white background on Windows.

$\% \quad$ See ISPC and COMPUTER.

if ispc \&\& isequal(get(hObject,'BackgroundColor'), get( 0, 'defaultUicontrolBackgroundColor')) set(hObject,'BackgroundColor','white');

end 


\section{Create and Simulate LTI Model:}

\section{File: SimulateLTIModel.m}

Description: Brings up dialog for importing plant input/output data. Creates LTI model transfer function. Simulates output of model in the time-domain and plots the model output and plant output. Calculates the mean squared error, error \% and R-Squared values.

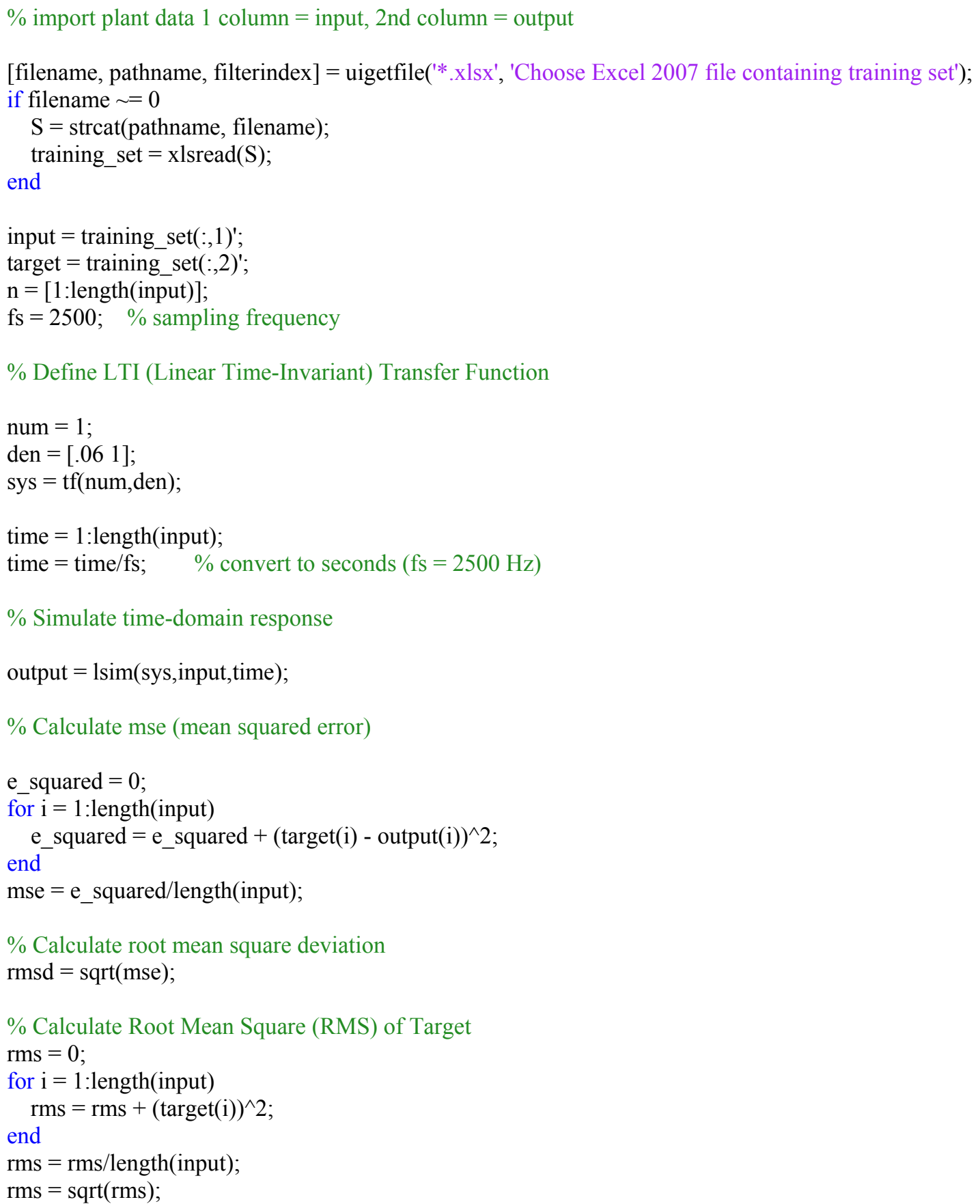


$\%$ Calculate error $=$ Root mean squared error $/$ RMS target $* 100 \%$

error $=\mathrm{rmsd} / \mathrm{rms} * 100$;

$\%$ Calculate $\mathrm{R}$ squared value

$\mathrm{r}=\operatorname{corr}($ target', output);

r_squared $=r^{\wedge} 2$;

$\%$ plot results

figure;

subplot(2,1,1), plot(time,input);

$\mathrm{h}=\mathrm{gca}$;

set(h, 'FontWeight','bold','FontSize',16);

title(\{['Plant/Model Input']; ['(Sampling Frequency fs = ',num2str(fs),'

Hz)']\},'FontWeight','bold','FontSize',16);

xlabel('Time (seconds)');

ylabel('Voltage (volts)');

subplot(2,1,2), plot(time,output,'b-',time,target,'g--','LineWidth',2);

legend('Model Output','Target','EastOutside','FontSize',16);

xlabel(\{'Time (seconds)';' ';['model numerator coefficients = ', num2str(num)];['model denominator coefficients = ', num2str(den)]\},'FontWeight','bold','FontSize',16);

ylabel('Rate (rad/s)','FontWeight','bold','FontSize',16);

title(\{['LTI Model Output and Plant Output (Target)']; ['( MSE = ', num2str(mse),' Error =

',num2str(error), '\%',' R-Squared = ',num2str(r_squared), ' )']\},'FontWeight','bold','FontSize', 16);

$\mathrm{h}=\mathrm{gca}$;

set(h, 'FontWeight','bold','FontSize',16); 